\title{
Towards a phylogeny of the Metazoa: evaluating alternative phylogenetic positions of Platyhelminthes, Nemertea, and Gnathostomulida, with a critical reappraisal of cladistic characters
}

\author{
Ronald A. Jenner \\ University Museum of Zoology, University of Cambridge, Downing Street, Cambridge CB2 3EJ, \\ United Kingdom
}

Keywords: Platyhelminthes, Nemertea, Gnathostomulida, Metazoa, Bilateria, phylogeny, morphological cladistics, 18S rDNA, total evidence

\begin{abstract}
"Our knowledge of the anatomy, and especially of the development, of the Invertebrata is increasing with such prodigious rapidity, that the views of Taxonomists in regard to the proper manner of expressing that knowledge by classification are undergoing, and, for some time to come, are likely to undergo, incessant modifications."
\end{abstract} - T.H. Huxley (1888: 561) -

\begin{abstract}
This paper critically assesses all morphological cladistic analyses of the Metazoa that were published during the last one and a half decades. Molecular and total evidence analyses are also critically reviewed. This study focuses on evaluating alternative phylogenetic positions of the 'acoelomate' worms: Platyhelminthes, Nemertea, and Gnathostomulida. This paper consists of two parts. In Part I, all recently proposed sister group hypotheses and the supporting synapomorphies for these phyla are evaluated. Discrepancies in the treatment of corresponding characters in different cladistic analyses are identified, and where possible, resolved. In Part II, the overall phylogenetic significance across the Metazoa of all characters relevant for placing the 'acoelomate' worms is examined. The coding and scoring of these characters for other phyla are evaluated, and uncertainties in our understanding are pointed out in order to guide future research. The characters discussed in this paper are broadly categorized as follows: epidermis and cuticle, reproduction and sexual condition, development, larval forms, coeloms and mesoderm source, nervous system and sensory organs, nephridia, musculature, digestive system, and miscellaneous characters. Competing phylogenetic hypotheses are compared in terms of several criteria: 1) taxon sampling and.the fulfillment of domain of definition for each character; 2) character sampling; 3) character coding; 4) character scoring and quality of primary homology; 5) quality of the proposed diagnostic synapomorphies as secondary hornologies. On the basis of this study I conclude that a sister group for the Platyhelminthes has not yet been unambiguously established. A clade minimally composed of Neotrochozoa (Mollusca, Sipuncula, Echiura, Annelida) emerges as the most likely sister group of the Nemertea on the basis of morphological and total evidence analyses. Finally, morphological data currrently favor a sister group relationship of Gnatho-
\end{abstract}

stomulida and Syndermata (probably plus Micrognathozoa). In contrast, molecular or total evidence analyses have not identified a reliable sister group of Gnathostomulida. Further progress in our understanding of metazoan phylogeny crucially depends on the improvement of the quality of currently adopted cladistic data matrices. A thorough reassessment of many of the more than 70 morphological characters discussed here is necessary. Despite the recent compilation of comprehensive data matrices, the power to test competing hypotheses of higher-level metazoan relationships is critically compromised due to uncritical data selection and poor character study in even the most recently published cladistic analyses.

\section{Contents}

Introduction

How to construct an effective cladistic test of competing phylogenetic hypotheses?

Completing the cladistic research cycle: beyond cladogram topology

The paradox of metazoan cladistics: data matrix compilation and cladogram construction

Molecular phylogenetics, developmental genetics, and body plan evolution

Goals and limitations of this study

Abbreviations and source trees

Higher level taxa and phyla

Part I: Comparing alternative phylogenetic hypotheses for the 'acoelomate' worms

1. Alternative phylogenetic hypotheses for Platyhelminthes

Platyhelminthes - Gnathifera Platyhelminthes - Gnathostomulida

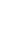




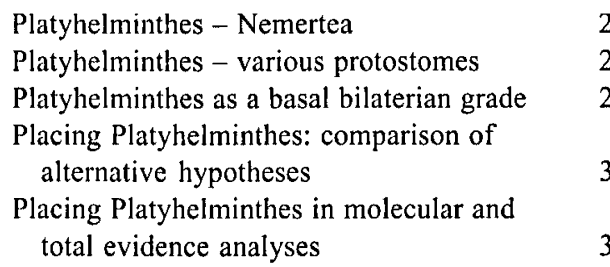

2. Alternative phylogenetic hypotheses for

Nemertea

Nemertea - Platyhelminthes 38

Nemertea - Annelida $\quad 38$

Nemertea - Entoprocta + Lobatocerebromorpha

Nemertea - 'Teloblastica'

Nemertea - 'Neotrochozoa'

Placing Nemertea: comparison of alternative hypotheses

Placing Nemertea in molecular and total evidence analyses

3. Alternative phylogenetic hypotheses for

Gnathostomulida

Gnathostomulida - Platyhelminthes

Gnathostomulida - Gastrotricha

Gnathostomulida - Syndermata

Gnathostomulida - Cycloneuralia

Gnathostomulida - Lobatocerebrum + Nemertea + 'Trochozoa'

Placing Gnathostomulida: comparison of alternative hypotheses

Placing Gnathostomulida in molecular and total evidence analyses

Part II: Character evaluations

Epidermis and cuticle

Reproduction and sexual condition

Development

Larval characters

Body cavities

Nervous system and sensory organs

Nephridial characters

Muscles

Digestive system

Miscellaneous characters

Conclusions: progress and prospects

Acknowledgements

References

\section{Introduction}

The literature on the evolutionary relationships of the Metazoa is immense and furnishes a plethora of alternative phylogenetic scenarios. Recent studies are chiefly based upon the analysis of $18 \mathrm{~S}$ rDNA sequences and morphology, which offer the most comprehensive data sets for higher level animal phylogenetics currently available. The use of comprehensive morphological data sets in all recent phylogenetic analyses indicates that morphological evidence continues to play a key role in the reconstruction of metazoan deep history, and I primarily focus on morphological cladistic analyses in this paper.

The advent of computer assisted cladistic analyses of explicit morphological data matrices has provided a marked improvement in the repeatability and testability of phylogenetic analyses. Furthermore, it has provided a means to evaluate and test the many, largely all too narrative attempts to reconstruct the genealogy of the animal kingdom that have dominated the field until recently. However, despite these developments a rigorous morphology-based consensus of animal relationships is not yet apparent (compare Nielsen et al., 1996; Zrzavý et al., 1998, 2001; Jenner \& Schram, 1999, 2002; Giribet et al., 2000; Nielsen, 2001; Peterson \& Eernisse, 2001; Zrzavý, 2003).

This contribution is an attempt to further our progress towards constructing such a synthesis by critically assessing the empirical and interpretational basis of recently proposed phylogenetic hypotheses. A new cladistic analysis is not attempted here because I feel that further progress in our understanding of metazoan phylogeny currently pivots around improving the quality of available morphological data matrices. Only that will eventually allow the comprehensive testing of the many existing alternative hypotheses (Jenner \& Schram, 1999; Jenner, 2001a, b, 2002, in press).

The often profound discrepancies between recently published morphological cladistic analyses of metazoan phylogeny demand an explanation. By explicitly analyzing aspects of methodology, we previously attempted to locate an important source of these discordances in differences of data matrix construction, including character selection and coding, character scoring and weighting, ground pattern reconstruction, and taxon selection (Jenner \& Schram, 1999; Jenner, 2000, in press). Although a few selected examples were treated rather briefly in these papers, the work herein is intended as the first in a series of papers that together will provide a comprehensive evaluation of contemporary competing phylogenetic hypotheses of higher level meta- 
zoan taxa. This paper focuses on the phylogenetic placements of the so-called 'acoelomate' worms: Platyhelminthes, Nemertea, and Gnathostomulida.

The following section will provide a sketch of the developments in comparative invertebrate zoology that have coalesced to form the impetus of this paper. These are as follows. 1) Despite the compilation of large data sets, it can be shown that cladistic analyses of the Metazoa have so far typically been performed without sufficient attention to the testing of competing phylogenetic hypotheses (Jenner, in press; this paper). 2) The overly restricted focus on cladogram topology in most recent studies masks the signs of true advances in our understanding of metazoan phylogeny and character evolution. 3) Recent cladistic analyses of the Metazoa show an imbalance between the amount of effort invested in the careful construction of the data matrix, and the cladistic analysis of that data set, with the former deserving more attention than is current practice (Jenner, 2001a, 2002, in press). 4) The "new view" of animal relationships emanating from molecular systematic studies motivates the reappraisal of many morphological synapomorphies, and a robust morphological data base is essential for the formulation of hypotheses of body plan evolution within the framework of this molecular phylogenetic backbone.

\section{How to construct an effective cladistic test of com- peting phylogenetic hypotheses?}

Acknowledging the existence of an overwhelming array of alternative hypotheses of animal relationships, it is striking how many recent cladistic analyses have been chiefly concerned with the proposal of 'new' hypotheses, rather than with the rigorous and comprehensive testing of the myriad existing alternatives. Naturally, one cannot draw a sharp line between performing a new phylogenetic analysis and testing an existing hypothesis, because any independent analysis can in principle be interpreted as a test of any other analysis. However, it is critical to understand the importance of careful attention to the key ingredients that will determine the testing power of a cladistic analysis, namely the selection of taxa and characters, and the coding and scoring of characters.

A cladistic test subjects primary homology propositions to a character congruence test that has the potential to separate corroborated (secondary) homologies, and refuted homologies, i.e., homoplasies. I would argue that the congruence test represents the primary advance of cladistics over traditional phylogenetic studies that restricted the analysis to tests of anatomical similarity (Patterson, 1982, 1988). The added value of a phylogenetic analysis based on a congruence test resides in the potential to refute or corroborate alternative hypotheses of homology and relationships by allowing the selection of the hypothesis that maximizes character congruence and thus minimizes the need for ad hoc explanations of the data, i.e., homoplasies. However, it is misleading to think that all results of a cladistic study have necessarily been directly subjected to a character congruence test.

"Refutation (falsification) [of a cladistic hypothesis] resides in incongruent synapomorphies..." (Kluge, 1997: 86), and "the strongest test of a hypothesis is the acquisition and incorporation of more data. Specifically, more taxa or more characters" (Siddall \& Whiting, 1999: 21). Incorporation of all relevant data constitutes the most "severe" test of a phylogenetic hypothesis. It can lead to provisional acceptance of the most corroborated hypothesis that embodies maximal explanatory power (Kluge, 1997). A cladistic study will only operate as an effective test, however, when the input data in principle allows the refutation or corroboration of either a particular phylogenetic hypothesis, or primary homology proposition. If the information in the data matrix cannot in principle vindicate all relevant alternative hypotheses, then the analysis could inspire false confidence in its results (based on circular reasoning; Lee, 1998a, 1999). In other words, if the input data is biased a priori towards accepting a particular phylogenetic hypothesis, for example through restrictive character or taxon sampling, then the outcome of a cladistic study may be decided prior to the congruence analysis. Consequently, the pattern of relationships that emerges from such a study may in fact be an untested assumption, not a new conclusion. 
This important distinction generally has not received attention in the current literature on higher level animal relationships, even in those studies that employ the most comprehensive data matrices compiled to date (see this study and Jenner, 2001a, in press). This is so despite the importance of being able to recognize a cladistic analysis as either an effective test of conflicting hypotheses, or as a mere summary of uncontested data. It can thus be summarized that "the issue is how honestly the relevant data are surveyed for those synapomorphies that actually have the potential to refute a cladistic hypothesis, those synapomorphies that can count as independent ad hoc hypotheses of homoplasy" (Kluge, 1998: 350).

One example from the recent literature will illustrate how proposed phylogenetic hypotheses based on a cladistic analysis may not be the corroborated results of a congruence test, but necessarily result from biased (not necessarily unjustified) data input. Further examples and a general discussion of hypothesis testing in contemporary metazoan cladistics is provided in Jenner (in press). In their comprehensive morphological phylogenetic analysis of the Metazoa, Nielsen et al. (1996) evaluated the relationships of Cnidaria and Ctenophora. Classically, these taxa are united as Coelenterata, a hypothesis that still finds support in some recent works (Ax, 1989; Ruppert \& Barnes, 1994), although it has been largely abandoned in most other recent phylogenetic studies. Nielsen et al. (1996: 397) report with respect to their own analysis that "... the sister group status of the two phyla is not supported by any of the computer generated analyses..." Consequently, the conclusion that the Coelenterata hypothesis failed a proper character congruence test would appear to be justified. Yet, this would be a misleading conclusion. The data matrix of Nielsen et al. (1996) did not include any of the potential synapomorphies of Cnidaria and Ctenophora, such as 1) polar bodies formed at the blastoporal pole, 2) unipolar first cleavage running from the blastoporal pole towards the aboral pole, or 3) a medusa with tetraradial symmetry (Ax, 1989; Martindale \& Henry, 1998; Goldstein \& Freeman, 1997). So, unsurprisingly, Nielsen et al. (1996) uncovered no support for the Coelenterata hypothesis. Although the rationale (based on comparative morphology) of Nielsen et al. (1996) for excluding these potential synapomorphies may have been entirely valid, it would be incorrect to claim that the Coelenterata hypothesis has been subjected to, and subsequently been rejected on the basis of, a character congruence or cladistic parsimony test. In this case, the cladistic analysis has not contributed any extra information in addition to data selection to bear on the problem of alternative relationships of the cnidarians and ctenophores.

\section{Completing the cladistic research cycle: beyond cladogram topology}

"Research in phylogenetic systematics is necessarily cyclic, and the place where the positive shift in understanding occurs is subsequent to discovering the most parsimonious cladogram(s)." -Kluge (1998: 349)-

Kluge (1998) drew attention to the necessarily cyclical nature of cladistic research. One research cycle can be decomposed into different stages. Typically the first stage involves the selection of terminal taxa and characters, which leads to the construction of the data matrix. The second stage is the testing of all possible cladograms during the character congruence test (the cladogram building phase). A third phase may constitute the reappraisal of the initial characters used to construct the cladogram, in particular the homoplasies incongruent with the hypothesis, or the performance of a test of consilience with independent data. It is during this third phase that the weight of evidence for the hypothesis (cladogram) is assessed. These considerations may then subsequently be fed into a new cladistic analysis, for example as experimental modifications of character coding, for further testing of taxon relationships and character homologies. It should be noted that the second stage of the research cycle is largely automated, and all scholarship instead resides in the first and third phases of the cladistic research cycle.

It can be argued that current practice in metazoan cladistics has not fully completed the cladistic research cycle. As is shown in Jenner (2001a, 2002 , in press), and in the present paper, it is espe- 
cially the first and third stages of the research cycle that should be significantly improved. For example, although some attention is directed towards the third stage of cladistic research, the analyses mostly direct their attention towards the comparison of cladogram topologies with only minimal attention to character evidence. Topological congruence between phylogenies derived from different data sets is generally considered as one of the strongest supports for phylogenetic relationships, and topological congruence is the logical foundation for all consensus methods widely used in systematic biology including the construction of supertrees (Cunningham, 1997; Sanderson et al., 1998). Accordingly, it is understandable that discussions of progress in metazoan phylogenetics focus on topological congruence in virtually all published cladistic analyses. A focus on tree topology allows the results of each new study to be concisely summarized and compared, and to be communicated in a straightforward manner. However, there is reason to believe that the prevailing and extreme focus on mere topological congruence without detailed attention to underlying data has created a false view of consensus in the field, and has in fact stymied true progress in understanding metazoan relationships and character evolution. The locus for a positive shift in understanding in phylogenetic systematics is not restricted to tree topology. One of the most important goals of cladistic analyses is to attempt to understand the link between evidence and hypothesis, which can only be achieved through detailed (re)assessment of the characters underpinning the cladograms.

Consistent with the current penchant in metazoan cladistics for quantitative rather than qualitative support measures for cladistic hypotheses, cladogram robustness is typically assessed by support statistics, such as bootstrap percentages or Bremer support values. However, if unaccompanied by a qualitative study of character evidence, these quantitative measures of support may be spurious. This problem is addressed in Jenner (2002), showing that the interpretation of character state transformations (steps) of almost $40 \%$ of the characters included in the most recent cladistic analyses of the Bilateria or Metazoa is seriously hampered due to character coding problems. Such difficulties are simply invisible to quantitative support measures.
In addition, the uncritical use of previously compiled data sets in new cladistic studies reveals that data reinterpretation does not receive adequate attention. Instead, many characters, or even entire data sets are uncritically recycled (Jenner, 2001a, this paper) resulting in the perpetuation of old mistakes in new studies. The present paper illustrates this for several characters, such as the gonocoel, cerebral ganglion, ventral nervous system, cuticle layering, and coelomocytes. Other authors do compile original data sets, however, without explicitly defending their selection of characters (Jenner, in press). The results are data sets that may differ markedly in the scope of included characters, however, frequently without explicit justification. This makes it virtually impossible to separate true progress in understanding from mere changes of opinion, even though a certain degree of topological congruence between studies is apparent. To enable future progress in morphological metazoan cladistics, and to complete the research cycle, workers will have to take previous work more explicitly into account when creating "new" results.

For example, in addition to manual cladistic analyses by Ax (1985, 1989, 1995), many comprehensive morphological cladistic analyses published during the last decade have yielded apparent support for a monophyletic Plathelminthomorpha (Platyhelminthes + Gnathostomulida), e.g., Schram (1991), Eernisse et al. (1992), Zzravy et al. (1998), Giribet et al. (2000), and Peterson \& Eernisse (2001). Judging purely on the basis of this topological congruence and the sheer number of proposed diagnostic synapomorphies, this clade appears to be a robust hypothesis. However, as will be shown in this paper, scrutiny of all proposed synapomorphies across all these studies, combined with in depth study of all proposed alternative hypotheses, leads to a very different conclusion. As we will see, careful character analysis shows that different studies supposedly providing independent support for a monophyletic Plathelminthomorpha are not even congruent among themselves. Clearly, the study of topological congruence is a necessary ingredient of metazoan phylogenetics, but it is not sufficient in "and of itself. 
The paradox of metazoan cladistics: data matrix compilation and cladogram construction

"Unfortunately, no one can be told what the matrix is. You have to see it for yourself." -Morpheus (in The Matrix, 1999, by A. Wachowski \& L. Wachowski)-

Nielsen (2001: 499) wrote that for higher level animal taxa the "choice and interpretation, i.e., coding, of characters pose enormous problems." It is then logical to expect that the compilation of the cladistic data matrices has received ample explicit attention in recent studies to ensure that the characters reflect organismic variation as accurately as possible. However, the reality is quite different. New cladistic analyses habitually copy characters or even entire data matrices from previous studies performed by different workers, often without any explicit restudy of the characters (Jenner, 2001a; this study). This is a problem because many of the recent cladistic data sets are riddled with errors (this study).

Furthermore, despite the fact that the ' 0 s' and ' $1 \mathrm{~s}$ ' in data matrices compiled for higher level taxa are typically far from unsullied observations, the explicit attention given to character coding in metazoan cladistics is minimal (Jenner, 2002). The most elaborate justification for an adopted coding strategy in the recent literature is given by Peterson \& Eernisse (2001: 173) who "acknowledge that these coding issues are contentious but feel that at the moment this [binary absence/presence coding] is the most conservative coding scheme available." This short "defense" is hardly compelling in view of the results of several recent investigations that have nominated absence/presence coding as the most problematic coding method available for standard cladistic analysis (see Jenner, 2002). Similarly, the justification for the scoring of characters also typically receives minimal documentation, with the scoring for only some of the included taxa being justified at best (see Peterson \& Eernisse, 2001; Zrzavý et al., 1998; Zrzavý et al., 2001). It then becomes noteworthy that for many characters shared between different cladistic analyses scoring conflicts for various taxa can be identified (this paper). It can thus be concluded that current practices belie the prime importance of the data matrix as the sole empirical anchor of cladistic analyses. Paradoxically, a striking contrast emerges when one compares the efforts to construct a reliable data set with the intensive efforts exerted in the extraction of phylogenetic signal from a given matrix. This paper is largely an attempt to redress the balance between character analysis and cladogram construction.

It is crucial to recognize phylogenetic research as an iterative process, where published data matrices provide the opportunity for restudy and reevaluation of the included data, and the critical comments offered in this paper should accordingly be interpreted as constructive rather than destructive. In this context, it is noteworthy that serious concerns about the quality of cladistic data matrices traverse a broad spectrum of taxa that range from parasitic flatworms to fishes (see Jenner, in press for references). Unfortunately, doubt about the quality of cladistic data matrices in general has inspired some authors to espouse depreciative conclusions about the objectivity of cladistic analyses, and some of our own work (Jenner \& Schram, 1999) has been taken to support "the subjectivity of such studies" (Salvini-Plawen, 2000: 142). This necessitates some brief comments on subjectivity and objectivity in cladistic analyses.

Biologists unanimously agree that organisms must be understood as integrated wholes. This realization creates a problem for phylogeneticists who wish to perform a cladistic analysis of organismic morphology, since this requires the subdivision of the organism into a set of well-demarcated and independent variables known as characters. The difficulty of precisely delineating 'a character' in the face of character correlations dictated by functional and structural interactions among body parts makes character coding one of the most critical and contentious steps in any cladistic study. If we deal with complex characters there may be several ways in which the observed organismic variation can be partitioned into cladistic characters. This opens a large range of coding possibilities that will have their own specific effects on the outcomes of phylogenetic analyses (see examples in Jenner, 2002 and this paper). The choice of a particular coding method is necessarily subjective. This realization has resulted in a limited but very important litera- 
ture that deals specifically with the relative strengths and weaknesses of different coding strategies (Poque \& Mickevich, 1990; Pleijel, 1995; Wilkinson, 1995; Hawkins et al., 1997; Lee'\& Bryant, 1999; Strong \& Lipscomb, 1999; Hawkins, 2000; Forey \& Kitching, 2000). Although the implications of these studies are currently being actively debated, the importance of these studies is nevertheless obvious. However, the results of these investigations have not yet permeated into the general consciousness of metazoan phylogeneticists (Jenner, 2002).

Despite the undeniable subjectivity of any statement or decision made by a single person, the objectivity of cladistic knowledge-claims is based on both the explicitness and transparency of cladistic data matrices, and the possibility of testing cladistic hypotheses by bringing new empirical data to bear on the problems at hand (Kluge, 1997, 1998; Ax, 1999). This potential to corroborate or refute a cladistic hypothesis (by inter-subjective testing; Ax, 1999) fully justifies the claim of objectivity of cladistic analyses.

\section{Molecular phylogenetics, developmental genetics, and body plan evolution}

Molecular phylogenetics of the Metazoa is currently dominated by analyses of $18 \mathrm{~S}$ rRNA/DNA sequences (see Giribet, 2002 for examples of other molecules used for metazoan phylogenetics). This has yielded a view of animal evolution different from the various morphology-based hypotheses (see Aguinaldo et al. 1997; Aguinaldo \& Lake 1998; Balavoine 1998; Balavoine \& Adoutte 1998; Maley \& Marshall 1998; McHugh 1998; Garey \& SchmidtRhaesa 1998; Halanych 1998; Winnepenninckx et al., 1998; Zrzavý et al., 1998; Adoutte et al. 1999, 2000; Knoll \& Carroll 1999; Ruiz-Trillo et al., 1999; Giribet et al., 2000; Peterson \& Eernisse, 2001). The two large protostomian clades, Ecdysozoa and Lophotrochozoa, have almost instantly become household names and flag bearers for this new view of animal evolution. Even the most comprehensively sampled molecular phylogenies show a lot of unresolved relationships, or arrangements that are puzzling from a morphological perspective such as the non-monophyly of morphologically supported taxa such as Mollusca and Nemertea (Giribet et al., 2000; Peterson \& Eernisse, 2001). Nevertheless, the emerging picture of animal evolution necessitates some striking re-examinations of 'received wisdom' based on comparative morphology. For example, the molecular view suggests convergent evolution in body plan features that have been traditionally imbued with great phylogenetic significance such as larval forms, body cavities, body segmentation, and excretory systems. Although these re-evaluations do not go unchallenged (Lüter \& Bartolomaeus, 1997; Wägele et al., 1999; Wägele \& Misof, 2001; Jenner, 1999, 2000), molecular systematics has at the very least provided a new set of hypotheses that encourage a detailed restudy of morphological characters. One of the most exciting developments of the past two decades has been the discovery of the underlying molecular mechanisms-of animal form. A wealth of new comparative data has emerged with tantalizing suggestions for animal body plan evolution. Comparative molecular developmental studies have provided the first glimpses of the genomic toolkit that might have been employed by the ancestor of the Bilateria (Carroll et al., 2001; Erwin \& Davidson, 2002), and the genetic underpinnings of several morphological features shared by many bilaterians have become a subject of intense study. These studies range from the use of Hox genes in primary and secondary larvae (Peterson et al., 2000a), the development of bilaterian nervous systems and photoreceptors (Arendt \& Nübler-Jung, 1996, 1999a; Arendt \& Wittbrodt, 2001; Arendt et al., 2002), vertebrate neural crest cells (Holland \& Holland, 1998), and the thyroid gland (Venkatesh et al., 1999). However, the complex nature of gene regulatory networks and hierarchies cautions against uncritical homology inferences of morphologies that are developmentally regulated by similar genes (Scholtz et al., 1998; Holland \& Holland, 1999; Janies \& DeSalle, 1999; Erwin \& Davidson, 2002). Nevertheless, careful study of developmental gene expression patterns may shed light on the nature of morphological characters (see for example below under Secondary body cavity, coelom: Ontogenetic source; Cerebral ganglion, brain), or suggest new synapomorphies, for example brachyury expression in the developing mesocoel/hydrocoel of the hemi- 
chordates and echinoderms (Peterson et al., 1999), and the expression of engrailed, Distal-less, and Hox genes in the study of homologies across the arthropods (Scholtz, 2001). Because the genetic underpinnings of most of the morphological characters used in metazoan cladistics have not yet been elucidated, there lies great promise in this field of research.

\section{Goals and limitations of this study}

This contribution is divided into two parts. Part I: Comparing Alternative Phylogenetic Hypotheses adopts a narrow focus. It is strictly concerned with the value of the character supports for competing phylogenetic hypotheses for placing Platyhelminthes, Nemertea, and Gnathostomulida within the Metazoa. Part II: Character Evaluations adopts a broader focus. It compares the treatment of the characters discussed in Part I across different cladistic analyses, and thereby assesses their broader phylogenetic significance across the Metazoa.

In summary, the goals of this study are:

(1) to provide the first comprehensive evaluation of competing phylogenetic hypotheses for Platyhelminthes, Nemertea, and Gnathostomulida by focusing on the quality of supporting data

(2) to provide a guide for future morphological cladistic analyses by identifying and, where possible, resolving conflicts in character coding and scoring between different analyses

(3) to identify characters upon which more research is needed for a better evaluation of their phylogenetic significance

(4) to provide a method for comparative biologists in search of a phylogenetic framework, or a morphological data set to study animal body plan evolution

This study is preliminary because:

(1) it is the first in a projected series of papers that will evaluate the phylogenetic positions of all animal phyla on the basis of morphological cladistic analyses
(2) it is the first necessary step towards the foundation for a novel cladistic study that will incorporate all points of discussion

(3) discussion is largely restricted to those characters that have been proposed as immediate synapomorphies for particular sister group relationships of the 'acoelomate' worms, although other phyla are discussed in Part II in order to resolved scoring conflicts of these characters between different studies

(4) discussion is restricted to cases of scoring conflict between different studies, identified misscorings, or instances of special difficulties of interpretation. No attempt was made to check every identifiable data matrix entry

\section{Abbreviations and source trees}

The following is a list of abbreviations, and of characters and phylogenies discussed in this paper and their sources.

AXX: character XX in Ahlrichs (1995) (manual cladistic analysis, numbered apomorphy sets on his fig. 92)

AhXX: character XX in Ahlrichs (1997) (manual cladistic analysis, fig. 6)

BXX: character XX in Brusca \& Brusca (1990) (computer-assisted cladistic analysis, fig. 1, chapter 24)

BIXX: character XX in Brusca \& Brusca (2003) (computer-assisted cladistic analysis, fig. 24.1, strict (consensus)

CXX: character XX in Christoffersen \& Araújode-Almeida (1994) (manual cladistic analysis, fig. 2)

CaXX: character XX in Carlson (1995) (computerassisted cladistic analysis, fig. 3, single MPT) EXX: character XX in Eernisse et al. (1992) (computer-assisted cladistic analysis, fig. 4 , strict consensus, + appendix 2)

EsXX: character XX in Ehlers \& Sopott-Ehlers (1997) (manual cladistic analysis, fig. 1)

GXX: character XX in Garey et al. (1998) (manual analysis, fig. 6)

HXX: character XX in Haszprunar (1996a) (computer-assisted cladistic analysis, fig. 1.1B, preferred 
MPT after unequally weighting)

HaXX: character XX in Haszprunar (1996b) (manual cladistic analysis, fig. 2)

KXX: character XX in Kristensen \& Funch (2000) (manual cladistic analysis, fig. 47)

LXX: character XX in Littlewood et al. (1999a) (computer-assisted cladistic analysis, fig. 2a, strict consensus)

MXX: character XX in Meglitsch \& Schram (1991) (computer-assisted cladistic analysis, fig. 38.2, single MPT)

MeXX: character XX in Melone et al. (1998) (computer-assisted cladistic analysis, fig. 1 single MPT) NXX: character XX in Nielsen et al. (1996) (computer-assisted cladistic analysis, fig. 2, preferred MPT)

NIXX: character XX in Nielsen (2001) (computerassisted cladistic analysis, fig. 56.1, strict consensus)

PXX: character XX in Peterson \& Eernisse (2001) (computer-assisted cladistic analysis, fig. 1, strict consensus)

RXX: character XX in Rouse (1999) (computerassisted cladistic analysis, fig. $3 \mathrm{~b}$, strict consensus after successive app. weighting)

RIXX: character XX in Rouse \& Fauchald (1995) (computer-assisted cladistic analysis, fig. 3, strict consensus)

SXX: character XX in Sørensen et al. (2000) (computer-assisted cladistic analysis, fig. 2, preferred MPT)

WXX: character XX in Wheeler et al. (1993) (computer-assisted cladistic analysis, fig. 5 , strict consensus)

WaXX: character XX in Wallace et al. (1996) (computer-assisted cladistic analysis, fig. 1, strict consensus)

ZXX: character XX in Zrzavý et al. (1998) (computer-assisted cladistic analysis, fig. 1, strict consensus after successive approximations weighting) ZIXX: character XX in Zrzavý et al. (2001) (computer-assisted cladistic analysis, fig. 1a, strict consensus)

ZIIXX: character XX in Zrzavý (2003) (computerassisted cladistic analysis, fig. 2 , strict consensus)

a/p: absence/presence

MPT: most parsimonious tree
TBR: tree bisection and reconnection branch swapping (heurstic search)

ACCTRAN: accelerated transformation (favors reversals over convergence in optimizing characters on a cladogram)

The recent morphological cladistic analyses of Giribet (1999) and Giribet et al. (2000) yielded identical results, and both studies used the morphological data matrix compiled by Zrzavý et al. (1998). Accordingly, all comments on the morphological analysis of Zrzavý et al. (1998) are applicable to these two studies. Similarly, all comments on the analysis of Meglitsch \& Schram (1991) are applicable to the studies that reanalyzed their data matrix: Eernisse et al. (1992), Rouse \& Fauchald (1995), Backeljau et al. (1993), and Wallace et al. (1996).

\section{Higher level taxa and phyla}

Although the logic implicit in cladistics does not support rank equivalence of traditional higher level Linnaean categories that bear the same label, such as phyla, classes, and genera, I will continue to use the term phylum as a general descriptor of higher level taxa without any Linnaean rank connotations.

The phylogenetic literature of the Metazoa is strewn with a confusing multitude of taxonomic names for higher level clades. The lack of agreement on the precise delimitations of these supraphyletic groups between authors often defies any straightforward identification of members in often used taxa such as Spiralia and Deuterostomia. Therefore, I find there is merit in providing here a synopsis of at least those supraphyletic taxa that are frequently referred to throughout this paper. The following list is neither complete, nor do the definitions adopted here necessarily correspond to those favored by most authors. Because suprafamilial taxa are not subject to the International Code for Zoological Nomenclature, and in view of the recent flux of ideas on the desirability of substituting the traditional Linnaean nomenclature by a phylogenetic nomenclature (Cantino et al., 1999), I have "chosen to design the following supraphyletic taxa for ease of use in the discussions in this paper without attention to historical source, exact nature of the 
original definitions, or the monophyly of the taxa, but never departing far from their original and most familiar meanings. Departures of the membership of supraphyletic groupings from the adopted definitions are noted in the appropriate place.

Acrosomata: Ctenophora, Bilateria

Aschelminthes: Cycloneuralia sensu Nielsen, 2001, Rotifera (incl. Seison), Acanthocephala, Micrognathozoa (Limnognathia maerski), Chaetognatha

Bilateria: Protostomia, Deuterostomia

Cephalorhyncha sensu Nielsen, 2001 (=Scalidophora sensu Lemburg, 1995): Priapulida, Kinorhyncha, Loricifera

Cycloneuralia sensu Nielsen, 2001 (= Nemathelminthes sensu Neuhaus, 1994): Gastrotricha, Nematoda, Nematomorpha, Kinorhyncha, Loricifera, Priapulida

Deuterostomia: Chordata, Hemichordata (Enteropneusta, Pterobranchia), Echinodermata

Diploblastica (Coelenterata, Radiata): Cnidaria, Ctenophora

Ecdysozoa: Panarthropoda, Introverta

Eumetazoa (Gastraeozoa, Histozoa): Cnidaria, Ctenophora, Bilateria

Euspiralia: Spiralia excluding Plathelminthomorpha Eutrochozoa: Nemertea, Mollusca, Sipuncula, Echiura, Annelida

Gastroneuralia: Spiralia, Aschelminthes

Gnathifera: Syndermata, Gnathostomulida, Micrognathozoa (Limnognathia maerski)

Hemichordata: Enteropneusta, Pterobranchia

Introverta: Nematoda, Nematomorpha, Kinorhyncha, Loricifera, Priapulida

Lophophorata (Tentaculata): Phoronida, Brachiopoda, Ectoprocta

Lophotrochozoa: Spiralia, Gastrotricha, Lophophorata

Nematoida: Nematoda, Nematomorpha

Neotrochozoa: Sipuncula, Echiura, Annelida, Mollusca

Panarthropoda: Arthropoda, Tardigrada, Onychophora

Plathelminthomorpha: Platyhelminthes, Gnathostomulida

Platyzoa: Gnathostomulida, Cycliophora, Syndermata, Gastrotricha, Platyhelminthes

Protostomia: Bilateria minus Deuterostomia
Schizocoelia: see Teloblastica

Spiralia: Annelida, Echiura, Sipuncula, Gnathostomulida, Mollusca, Nemertea, Entoprocta, Platyhelminthes, Panarthropoda

Scalidophora: see Cephalorhyncha.

Syndermata: Rotifera (incl. Seison), Acanthocephala

Teloblastica: Sipuncula, Mollusca, Annelida, Echiura, Onychophora, Arthropoda, Tardigrada

Trochozoa: Sipuncula, Echiura, Annelida, Mollusca, Entoprocta

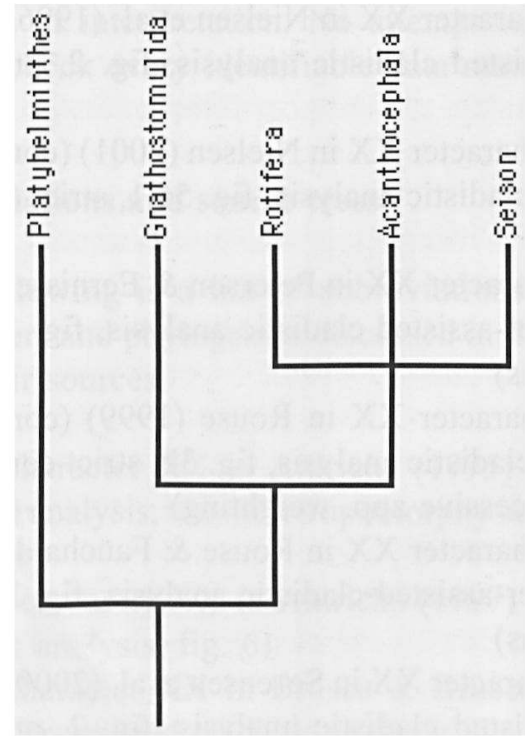

Fig. 1. Phylogenetic position of the platyhelminths and gnathostomulids according to Ahlrichs (1995), Garey et al. (1998), and Melone et al. (1998).

Part I: Comparing alternative phylogenetic hypotheses for the 'acoelomate' worms

I have devised a set of explicit criteria that I use as guidelines in the evaluation of competing sister group hypotheses:

(1) taxon sampling and fulfillment of domain of definition of each character. Is the range of selected terminal taxa broad enough to test all pertinent alternative hypotheses? I adopt a slightly modified version of the domain of definition as proposed by Dayrat \& Tillier 
(2000): the complete set of terminal taxa for which an included character is in principle applicable, irrespective of whether that character is uniformly present within all terminal taxa. A failure to fulfill the domain of definition of a character, i.e., restrictive taxon sampling, compromises the reliability of the phylogenetic significance ascribed to that character, so that it may be a symplesiomorphy or homoplasy rather than a unique synapomorphy.

(2) character sampling across studies.

(3) character coding. Are the adopted character states proper (natural) alternatives that cover the entire range of organismic variation, i.e., do the character states show a clear complement relation (Patterson, 1982, 1988)?

(4) quality of primary homology and character scoring. Are primary homologies properly identified, i.e., rooted in careful morphological analysis? Are the characters scored appropriately according to the adopted character definition? Is there consensus on the interpretation of the characters?

(5) quality of proposed diagnostic synapomorphies as secondary homologies. Are the proposed synapomorphies unique or homoplastic, reversals or convergences, losses or gains?

The combined consideration of the above criteria will provide an estimate of the relative quality of proposed hypotheses of metazoan phylogeny. The first four criteria represent the chief ingredients of all morphological cladistic analyses, and their combined input will determine the outcome of a cladistic analysis. The study of these ingredients will therefore allow one to infer the cause(s) of conflicting phylogenetic hypotheses, and to understand whether phylogenetic conclusions are the corroborated results of the balanced treatment of all pertinent information, or whether the results are unjustifiably biased. This information can serve as a basis for deciding which phylogenies may be dropped from consideration, and which hypotheses could profitably be explored in future studies.
1. Alternative phylogenetic hypotheses for Platyhelminthes

Many different phylogenetic placements have been proposed for the platyhelminths throughout the last century (see Tyler, 2001 for brief review), and the origin of their body plan has attracted recent attention in connection with the emergence of hypotheses for their placement on the basis of molecular sequence data (Balavoine, 1997, 1998; Jenner, 2000). Interestingly, 18S rDNA, myosin heavy chain type II (myosin II), and some mitochondrial gene (COI and $C y t b)$ data suggest that Acoela and Nemertodermatida are not in fact platyhelminths, but the earliest branching crown group bilaterians (RuizTrillo et al., 1999, 2002; Baguñà et al., 2001; Jondelius et al., 2002; Telford et al., in press). Apparent morphological support for this hypothesis was initially provided by Haszprunar (1996a, b), but all comprehensive morphological cladistic analyses with the exception of Zrzavý (2003) instead supported a position of platyhelminths nested within the protostomes either as a sister group to Nemertea, Gnathostomulida, or a larger clade of protostomes of variable membership. Several more restricted studies instead united the platyhelminths with gnathiferans. The following section evaluates the evidence for these competing hypotheses.

\section{(Platyhelminthes Gnathifera)}

Proposed synapomorphies: Ahlrichs (1995) (followed by Garey et al., 1998); Melone et al. (1998). See figure 1.

Ahlrichs (1995) (A13)

- internal sperm deposition and internal fertilization

- no mitosis in somatic cells

- filiform sperm

- lack of accessory centriole in sperm

\section{Comments}

Internal sperm deposition accompanied by internal fertilization is not a compelling synapomorphy of the platyhelminths and gnathiferans. The restricted taxon sampling of Ahlrichs (1995) does not allow 
the proper assessment of the phylogenetic significance of mode of sperm transfer and fertilization within the Metazoa, because these features (particularly internal fertilization) have a much wider distribution within the Bilateria than just the platyhelminths and gnathiferans. A further complication is introduced by the manual approach to cladogram construction adopted by Ah95. Application of the principle of parsimony leads to ambiguous results on Ahlrichs' cladogram. His cladogram is equally compatible with alternative hypotheses of character evolution, either interpreting internal insemination and fertilization as a gastroneuralian apomorphy (and hence a symplesiomorphy for platyhelminths and gnathiferans), or as a convergence between Platyhelminthes + Gnathifera and Cycloneuralia. For further details on these characters see the relevant sections below under Reproduction and sexual condition.

The lack of mitosis in differentiated somatic cells may be a valid character that should be tested through the study of a wide range of metazoans. Indeed, other phyla that are thought to be eutelic or to possess a fixed number of differentiated somatic cells such as the nematodes should be closely studied (see below also under Lack of mitosis in somatic or epidermal cells [eutely]).

Possession of filiform sperm has also been suggested as an autapomorphy of Plathelminthomorpha by $A x(1985,1989,1995)$, Eernisse et al. (1992), and Zrzavý et al. (1998). However, the phylogenetic significance of this character in none of these studies is compelling (see discussion under Filiform sperm for details). First, Ahlrichs (1995) did not perform a numerical cladistic analysis and consequently did not find the globally most parsimonious solution. Filiform sperm can be observed in various other phyla, where it may represent the plesiomorphic form of sperm, or a commonly evolved convergence within different monophyletic taxa (see discussion under Filiform sperm for further details). Furthermore, the domain of definition of filiform sperm is clearly not fulfilled. Various taxa that are characterized by possession of filiform sperm such as the entoprocts (Nielsen, 1971; Mariscal, 1975; Nielsen \& Jespersen, 1997) and the cycliophorans (Funch \& Kristensen, 1997), are not included in the analysis of Ahlrichs (1995). When one considers the taxa included in Ahlrichs' analysis, a form of filiform sperm, which would clearly fall within his broad definition, is also present in Gastrotricha, which may be very closely related to the plathelminthomorphans and syndermates (Zrzavý et al., 1998; Giribet et al., 2000).

Likewise, the lack of an accessory centriole in sperm cells is an equally unconvincing synapomorphy. For example, both the gastrotrichs and kinorhynchs possess ciliated sperm that lack an accessory centriole (Ahlrichs, 1995). Moreover, lack of an accessory centriole in sperm is probably correlated with the evolution of modified or filiform sperm, and this does not warrant the coding of an independent character. For example, the meiobenthic priapulid genus Tubiluchus possesses filiform sperm that probably evolved within the Priapulida (Storch et al., 2000), and it too is accompanied by the loss of an accessory centriole.

In conclusion, in order to approximate a globally most parsimonious solution, the distribution of Ahlrichs' characters would have to be re-assessed through a computer-assisted cladistic study of an expanded set of taxa.

Melone et al. (1998)

- statocysts present (Me60)

\section{Comments}

The use of statocysts as a synapomorphy for Platyhelminthes and Gnathifera is unconvincing. Curiously, Melone et al. (1998: 107) state that statocysts are primarily absent in Platyhelminthes. However, they are commonly found in the basal platyhelminth taxa such as Acoela, Nemertodermatida and Catenulida (Rieger et al., 1991b). Statocysts are widespread within the Metazoa, and for a proper fulfillment of the domain of definition more taxa have to be included in the analysis. More problematic, however, are the positive scorings of statocysts for the gnathiferans. So far, no unambiguous statocysts have been identified in any of these phyla (see under Statocysts). Expectedly, the sister group pairing of platyhelminths and gnathiferans in Melone et al. (1998) is a default result given the selection of platyhelminths as an out-group in a study that 



Fig. 2. Phylogenetic position of the platyhelminths, gnathostomulids, and nemerteans according to (A) Ax (1985, 1989, 1995) and Bartolomaeus (1993a); (B) Meglitsch and Schram (1991) and Schram \& Ellis (1994); (C) morphological analysis of Peterson \& Eernisse (2001); (D) Eernisse et al. (1992); (E) morphological analysis of Giribet et al. (2000); (F) morphological analysis of Zrzavý
et al. (1998). 
was primarily aimed at resolving phylogenetic relationships within Rotifera.

\section{(Platyhelminthes Gnathostomulida)}

This clade is known as Plathelminthomorpha. Proposed synapomorphies: Ax (1985, 1989, 1995; followed by Bartolomaeus, 1993a); Meglitsch and Schram (1991); Eernisse et al. (1992); Schram and Ellis (1994); Zrzavý et al. (1998); Giribet et al. (2000); Peterson \& Eernisse (2001). See figure 2.

Ax $(1985,1989,1995)$

- direct sperm transfer and internal fertilization

- filiform sperm

- no mitosis in somatic cells (Ax, 1995)

- hermaphroditism

\section{Comments}

Interestingly, the first three of these characters are also used by Ahlrichs (1995) to support an alternative sister group relationship between Platyhelminthes and Gnathifera (see above). This discrepancy in phylogenetic significance attributed to the same characters in Ahlrichs (1995) and Ax (1995) is easily explained by the observation that the manual cladistic analyses used in both studies focused on restricted sets of taxa (differing between the two studies), a method that tends to favor local over global parsimony. Consequently, although both studies use parsimony as their central principle, the most globally parsimonious solution for the characters in question can only result from a comprehensive study that includes all pertinent taxa at the same time (especially true for the first two widely distributed characters dealing with reproductive modes and sperm morphology). This conclusion also applies to the last synapomorphy proposed by Ax: hermaphroditism. Hermaphroditism is widespread within the Metazoa (see discussion under Sexes), and clearly the domain of definition of this character is not fulfilled in the various manual cladistic analyses by Ax. The study of Zrzavý et al. (1998) suggested multiple cases of convergent evolution of hermaphroditism, even though various taxa were misscored for the relevant character (Z126; see discussion below). Moreover, the value of hermaphroditism as a plathelminthomorphan autapomorphy is highly sensitive to cladogram topology. It may either be a highly homoplastic character between phyla (already well established within phyla, see under Sexes), or hermaphroditism may be a plesiomorphy for the plathelminthomorphans inherited from the last common ancestor of the Bilateria and the hermaphroditic ctenophores (sister taxa making up a monophyletic Acrosomata in the scheme of Ax, 1995). Rieger \& Tyler (1995), Haszprunar (1996b), and Kristensen \& Funch (2000) also argued against the plausibility of a monophyletic Plathelminthomorpha, principally by emphasizing the likelihood of convergence or plesiomorphy of the reproductive apomorphies.

Meglitsch \& Schram (1991)

- no anus (M16)

- no special muscle cells (M6)

- protonephridia (M31)

\section{Comments}

The secondary loss of an anus as a plathelminthomorphan autapomorphy is also found in Eernisse et al. (1992), Zrzavý et al. (1998), and Giribet et al. (2000). In contrast, Peterson \& Eernisse (2001) and Nielsen (2001) performed comprehensive cladistic studies that did not find this plathelminthomorphan support. Both these analyses considered an anus to be unambiguously present in gnathostomulids. The presence of a true anus in gnathostomulids homologous to those of other bilaterians is a contentious issue. Recent ultrastructural data (Knauss, 1979; Sterrer et al., 1985; Lammert, 1991) indicate a functional anus at the posterior end of the gut that consists of a tissue connection between dorsomedial epidermal cells and gut cells without a separating basal lamina. This morphology has at least been observed both in some filospermoidean and bursovaginoidean gnathostomulids. However, the phylogenetic significance of the singular morphology of this "anal pore" remains undecided. Recent phylogenetic analyses have divergently suggested that gnathostomulids either 1) unambiguously possess a complete, unidirectional gut with 
an anus that is homologous across Bilateria (Haszprunar, 1996b), 2) possess an autapomorphic anus (Haszprunar (1996a): H11; Zrzavý et al., 1998: Z72), 3) lack an anus either primitively (Ax, 1995), or 4) secondarily (Meglitsch \& Schram, 1991: M16; Wallace et al., 1996: Wa21), or 5) are polymorphic or uncertain for a complete gut (Littlewood et al., 1999a: L54; Melone et al., 1998: Me31; Zrzavý, 2003: ZII50). Interestingly, the recently described micrognathozoan Limnognathia maerski exhibits a very similar differentiation of the terminal end of the intestine, with interdigitating rectal and epidermal cells without an intervening basal lamina (Kristensen \& Funch, 2000, fig. 33).

The last two of the three characters found by Meglitsch \& Schram (1991) to support a monophyletic Plathelminthomorpha are problematic. The secondary loss of muscle cells in the platyhelminths and gnathostomulids is a spurious synapomorphy based on the misscoring of both taxa (in addition to gastrotrichs) for the absence of muscle cells. Furthermore, the character coding of M6 directly conflicts with the coding of the related character M13 so that taxa without muscle cells are nonetheless coded for either ectodermal or subepidermal muscles.

The presence of protonephridia is not a convincing plathelminthomorphan autapomorphy. First, protonephridia are widespread within the Metazoa, and they behave highly homoplastically in recent comprehensive phylogenetic studies (Nielsen et al., 1996; Zrzavý et al., 1998; Sørensen et al., 2000; Zrzavý, 2003). Moreover, any special similarity in protonephridial ultrastructure that could support monophyly of Plathelminthomorpha is lacking. Rather, the gnathostomulids share some similarities of protonephridial ultrastructure with several other phyla, including potentially closely related taxa, such as monociliate terminal cells with the gastrotrichs and Micrognathozoa (Ruppert, 1991b; Kristensen \& Funch, 2000). Second, although the presence of protonephridia in the platyhelminth ground plan is generally accepted, it should be noted that this determination hinges upon the phylogenetic position of the acoelomorphs (Acoela, Nemertodermatida) within Platyhelminthes, and of the Platyhelminthes within the Metazoa. The usual interpretation of the lack of protonephridia in the acoelo- morphs as a secondary loss (Rieger et al., 1991b; Ax, 1995), is largely dictated by the placement of the acoelomorphs within a clade of platyhelminths that otherwise all possess protonephridia. Interestingly, the morphological analysis of Littlewood et al. (1999a) (their figure 2b) placed acoelomorphs as the sister group to the remaining platyhelminths, thus making the interpretation of the evolutionary significance of the lack of protonephridia in acoelomorphs dependent upon out-group comparison. In striking contrast, the morphological cladistic analyses by Haszprunar (1996a, b) and Zrzavý (2003) placed the acoelomorphs as the earliest diverging branch of the bilaterians, consequently implying the lack of protonephridia as a plesiomorphy. This hypothesis is also supported by molecular data (Jondelius et al., 2002; Ruiz-Trillo et al., 2002; Telford et al., in press). However, Haszprunar's results should be regarded with caution since his phylogenetic analyses excluded many of the protostome phyla and all the deuterostomes.

In conclusion, Meglitsch \& Schram (1991) have not provided unambiguous support for the monophyly of Plathelminthomorpha, and their placement of the platyhelminths and gnathostomulids together within the Metazoa should therefore be regarded with due caution.

\section{Eernisse et al. (1992)}

- lack of anus with proctodeum (complete unidirectional alimentary canal) (E96)

- hermaphroditism (E117)

- filiform sperm (E118)

- direct internal fertilization (E119)

\section{Comments}

The computer-assisted analysis of Eernisse et al. (1992) appears to confirm the relevance of some of the features that were proposed to be important plathelminthomorphan autapomorphies by Ax (1985, 1989, 1995) and Meglitsch \& Schram (1991). However, do the results of Eernisse et al. (1992) really support plathelminthomorphan monophyly?

The secondary loss of an anus is also supported as a plathelminthomorphan autapomorphy by Meglitsch \& Schram (1991), Zrzavý et al. (1998), and Giribet et al. (2000), and it may indeed be a valid 
apomorphy when plathelminthomorphans are nested deeply within the Bilateria (see discussion above for more details). Including the proctodeum into the character definition is unnecessarily complicating. Gastrotricha and Micrognathozoa, for example, also lack a defined hindgut or proctodeum, although the gastrotrich ground pattern cannot be identified unambiguously (Ruppert, 1991b; Kristensen \& Funch, 2000). Many gastrotrichs and Limnognathia maerski also lack well-defined anuses.

For the last three characters Eernisse et al. (1992) did not fulfill the domains of definition. These characters apply to a range of excluded taxa as well. Eernisse et al. (1992) justified the scoring of presence of hermaphroditism when it was found in more than single isolated species. This would necessitate a change in scoring for the phoronids and nematodes, certainly when it is observed that Eernisse et al. (1992) did score the nemerteans and conchiferan molluscs polymorphic for hermaphroditism. The scoring of Solenogastres as gonochoristic is erroneous, since they are without exception simultaneous hermaphrodites (Scheltema et al., 1994). Equally important, apart from various excluded gonochoristic taxa, several hermaphroditic phyla that are either potentially closely related to plathelminthomorphans, such as the gastrotrichs and chaetognaths, or more distantly related, such as the ectoprocts and ctenophores, were excluded from the analysis of Eernisse et al. (1992). Clearly, increased taxon sampling is necessary for a better estimation of the phylogenetic significance of hermaphroditism.

As noted earlier, filiform sperm can be scored for a host of taxa not included in Eernisse et al. (1992) (see section under Filiform sperm), and several of the included taxa should be rescored, among others Solenogastres (Buckland-Nicks \& Scheltema, 1995), pogonophorans including vestimentiferans (Gardiner \& Jones, 1993; Southward, 1993, 2000), clitellates (Jamieson, 1992; Fernández et al., 1992), and onychophorans (Storch \& Ruhberg, 1993).

Several problems are apparent with the character direct internal fertilization. It combines two logically separate variables, namely mode of sperm transfer, here direct sperm transfer, hence direct internal fertilization, and mode of fertilization. Ax (1995) also listed both direct sperm transfer and internal fertilization as plathelminthomorphan autapomorphies (as did Ahlrichs, 1995 for his clade of Platyhelminthes + Gnathifera). However, mode of sperm transfer and fertilization exhibit no strict correlation (although a certain correlation cannot be altogether denied, see under Mode of sperm transfer and mode of fertilization), and could therefore be coded as separate characters. Moreover, the scoring observed in Eernisse et al. (1992) illustrates a rather subtle but important misunderstanding of the relation between mode of sperm transfer and fertilization, and between different modes of sperm transfer. The coding of E119 exhibits no complement relation, i.e., the alternative character states do not cover the entire range of organismic variation, making misscorings inevitable. The coding assumes that internal fertilization is always accompanied by direct sperm transfer. However, in view of the difference between indirect and direct sperm transfer (sperm deposited on outside of body or inside, respectively; see discussion under Mode of sperm deposition and mode of fertilization), various taxa have to be rescored, in particular the arthropods, which were subdivided into Crustacea, Uniramia (myriapods and insects), and Chelicerata. At least uniramians and chelicerates should be reassessed for their scoring of direct internal fertilization. External fertilization may be primitive for chelicerates in view of the frequently supported basal positions of pycnogonids and xiphosurans within the cheliceriform clade (Weygoldt, 1996; Ax, 1999; Wheeler \& Hayashi, 1998; Giribet et al., 2001, 2002; Waloszek \& Dunlop, 2002). However, the exact phylogenetic placements of these taxa within the Arthropoda remains a contentious issue (Edgecombe et al., 2000; Giribet \& Ribera, 1998, 2000; Giribet et al., 2001; Schram \& Jenner, 2001). Nevertheless, indirect sperm transfer is widespread within the remaining chelicerates, and copulation (direct sperm transfer) has evolved multiple times convergently (Schaller, 1979; Proctor, 1998). Similarly, within the uniramians (atelocerates), the myriapods and apterygote hexapods (insects) chiefly exhibit indirect sperm transfer, with copulation evolving at the base of the pterygotes (Wheeler et al., 2001). It is therefore likely that indirect sperm transfer is primitive for the myriapods and hexapods ( $\mathrm{Ax}$, 1999). However, a satisfactory understanding of 
the evolutionary changes in modes of sperm transfer within the Arthropoda will have to await the resolution of the continually perplexing problem of arthropod relationships (see Fortey \& Thomas, 1997; Richter, 2002). Interestingly, [and with immediate importance for the value of direct sperm transfer in uniting the gnathostomulids with the platyhelminths (Eernisse et al., 1992, Ax, 1985, $1989,1995)$, or the platyhelminths and the syndermates (Ahlrichs, 1995)] currently available evidence on the distribution of reproductive modes within Gnathostomulida strongly suggests that they possess indirect sperm transfer through hypodermic impregnation, rather than direct sperm transfer (Sterrer, 1972; Mainitz, 1989) (see under Mode of sperm deposition and mode of fertilization for a discussion of these features).

Finally, even if we allow a least restrictive character definition for E119 that retains the intended contrast between internal and external fertilization without distinguishing between direct and indirect sperm transfer, several misscorings for E119 remain. These include: Phoronida (characterized by internal fertilization in contrast to common opinion: Emig, 1990; Zimmer, 1991; 1997); Pogonophora (internal fertilization is indicated for pogonophorans, and suggested for at least one species of vestimentiferan: Southward, 1999), Solenogastres (possess internal fertilization: Buckland-Nicks \& Scheltema, 1995), and Clitellata. Despite the fact that many clitellates transfer sperm to their partner in copulation, in oligochaetes the fertilization nevertheless takes place outside the body in the secreted cocoon (Needham, 1989; Westheide, 1996). Evolutionary changes to internal fertilization have independently occurred in one family of oligochaetes (Eudrilidae, nested deeply within Oligochaeta: Jamieson, 1988), and in hirudineans (Jamieson, 1992; Fernández et al., 1992).

\section{Zrzavý et al. (1998)}

- filiform sperm (Z117)

- absence epidermal mitosis (Z191)

- lack of coeloblastula (Z11)

- lack of anus (Z72)

- sacular $\rightarrow$ asacular gonads (Z110)

- monoflagellate $\rightarrow$ biflagellate sperm (Z115)

- lack of compact acrosome (Z120)
- hermaphroditism (Z126)

- rare/absent $\rightarrow$ dominant asexual reproduction (Z128)

- two layered $\rightarrow$ simple cuticle (Z193)

- adult locomotion muscular $\rightarrow$ ciliary (Z258)

\section{Comments}

The phylogenetic analysis of Zrzavý et al. (1998) yielded 11 apomorphies in support of a monophyletic Plathelminthomorpha. However, closer inspection reveals that none of these are compelling, and most of the characters are misscored for various taxa.

Although filiform sperm is an unambiguous unique autapomorphy of Plathelminthomorpha in the morphological analysis of Zrzavý et al. (1998), its unambiguous or polymorphic presence can be scored for the ground patterns of various additional taxa (see detailed discussions under Eernisse et al. (1992) in this section and under Filiform sperm). Zrzavý et al. (1998) score this feature in a way (filiform sperm present only in the platyhelminths and gnathostomulids) that can only be explained by assuming that they uncritically adopted the scoring of $\mathrm{Ax}$ (1995), a study that aimed for local parsimony in the context of a restricted sample of taxa rather than attempting to document the global distribution of this character across the entire Metazoa.

The absence of epidermal mitosis was also suggested as a unique plathelminthomorphan autapomorphy. However, it is doubtful whether its value as a phylogenetic marker has been fully explored. For example, epidermal mitosis has also never been observed in the chaetognaths (Shinn, 1997), and the lack of mitosis in somatic cells has been scored for a broader range of taxa (Ahlrichs, 1995). Clearly, detailed study of an expanded set of taxa is needed (see below under Lack of mitosis in somatic or epidermal cells [eutely] for further discussion).

The lack of a coeloblastula is unconvincing since it is scored as "?" for several platyhelminth taxa as well as for the gnathostomulids.

The secondary lack of an anus may be a valid apomorphy, as was also suggested by the studies of Meglitsch \& Schram (1991) and Eernisse et al. (1992) (see discussions above).

The transformation from sacular to asacular go- 
nads is uncompelling since the gnathostomulids and platyhelminths do not evidently share a common ground pattern. Gnathostomulids are scored as possessing males with sacular gonads, while all platyhelminths except nemertodermatids are scored as having asacular gonads. Further difficulties with this character are the conflicting scorings for rhabditophoran platyhelminths in Zrzavý et al. (1998), Haszprunar (1996a), and the study to which this character can be traced, namely Rieger et al (1991b). Although Rieger et al. (1991b) and Rieger (1996a) report that sacular gonads are typical for virtually all rhabditophorans, save some prolecithophorans, Haszprunar (1996a) and Zrzavý et al. (1998) nevertheless incorrectly score rhabditophorans as possessing asacular gonads.

The change from monoflagellate to biflagellate sperm is invalid as a plathelminthomorphan autapomorphy. Although gnathostomulids were scored "?" for Z115, they possess either monoflagellate (considered typical), or aflagellate (atypical) sperm (Sterrer, 1972; Lammert, 1991). Biflagellate sperm has never been reported in the gnathostomulids, and it is a variable character within the platyhelminths.

The lack of a compact acrosome in sperm is convergent, and it appears to be associated with the presence of filiform sperm. Its value as a plathelminthomorphan autapomorphy is uncertain, however, since a compact acrosomal vesicle may be present in the tip of the filiform sperm of the filospermoid gnathostomulids (Sterrer et al., 1985, fig. 12.4a). Confirmation of this interpretation is needed.

Although hermaphroditism is characteristic of the plathelminthomorphans, hermaphroditism is also present in the ground patterns of many other phyla. The misscorings for this character in the matrix of Zrzavý et al. (1998) compromise its value as a phylogenetic marker. Taxa wrongly scored as being plesiomorphically hermaphroditic include Kinorhyncha (Kristensen \& Higgins, 1991), Priapulida (although occasional hermaphroditic individuals are known, Storch, 1991; Lemburg \& Schmidt-Rhaesa, 1999; Storch et al., 2000), and Nemertea (Turbeville, 1996; Norenburg \& Stricker, 2002). Nematomorpha (scored '?') can also be rescored as being gonochoristic (Bird \& Sommerville, 1989; Bresciani, 1991; Schmidt-Rhaesa, 1999), while Myzostomida (scored as 'gonochoristic') are hermaphrodites with the exception of a single species (Westheide, 1997; Grygier, 2000). Although Zrzavý et al. (1998) unambiguously scored the ectoprocts to be gonochoristic, this does not accurately characterize their sexual system. While all known ectoproct colonies are hermaphroditic, common gonochoristic zooids are mostly restricted to the stenolaemates. In light of this information, it should be noted that the terminal taxon Bryozoa (Ectoprocta) in Zrzavý et al. (1998) is solely comprised of the gymnolaemates and phylactolaemates. Zooids are typically hermaphroditic in the gymnolaemates and phylactolaemates, necessitating a rescoring of $\mathrm{Z} 126$, but different sources may give conflicting estimates for the relative frequency of hermaphroditic versus gonochoristic zooids: Nielsen (1990), Zimmer (1997), and Mukai et al. (1997) report that most gymnolaemates possess hermaphroditic zooids, while Reed (1991) reports that most gymnolaemates exhibit zooid gonochorism.

Abundant scoring problems among others for the platyhelminths and gnathostomulids reveal that dominant asexual reproduction is certainly not a reliable autapomorphy for Plathelminthomorpha. Because asexual reproduction has not been reported for any gnathostomulid, they should be rescored as having rare or absent asexual reproduction (they were scored'?'). Furthermore, although the nemertodermatids are scored for dominant asexual reproduction, without exception they reproduce sexually (Lundin \& Sterrer, 2001). Even though Macrostomorpha are unambiguously scored for having dominant asexual reproduction, their ground pattern state is actually rather uncertain since asexual reproduction by paratomy is only recorded for two of the thirteen major macrostomorphan taxa (Rieger, 2001), neither of which is the most basal macrostomorphan group. Despite Rieger's (2001) claim that paratomy was probably part of the macrostomorphan ground pattern, or even that of the Platyhelminthes as a whole (Ehlers, 1985), with four subsequent losses, it is more parsimonious to explain the distribution of paratomy in macrostomorphans by two cases of convergent evolution (phylogeny depicted in fig. 4.10 and 4.11 in Rieger, 2001). At the very least, the reconstruction of the primitive reproductive mode of the macrostomorphans is dependent upon out-group comparison. 
Since some of the potential out-groups are, and others are not characterized by asexual reproduction, and because recent comprehensive phylogenetic analyses (based on morphology, molecules or combined data) have not yet identified the most likely out-group for macrostomorphans (Littlewood et al., 1999a; Littlewood \& Olson, 2001; Joffe \& Kornakova, 2001), uncertainty about their ground pattern remains. Furthermore, the scoring of Z128 is puzzling for many other taxa as is discussed under Asexual/sexual reproduction. In conclusion, a thorough re-assessment of asexual/sexual reproduction is imperative.

The possession of a simple cuticle or glycocalyx by the plathelminthomorphans is shared with a range of other taxa, including the nemerteans, syndermates, and Micrognathozoa (Turbeville, 1991; Clément \& Wurdak, 1991; Dunagan \& Miller, 1991; Kristensen \& Funch, 2000), which have all been either closely associated with the platyhelminths or the gnathostomulids in other studies. Consequently, the possession of a glycocalyx or simple cuticle cannot discriminate between these alternative sister groupings. Furthermore, the coding of the alternative character state of a two-layered cuticle needs to be re-evaluated. Many of the taxa scored as possessing a two-layered cuticle actually have three or more layers in the cuticle, and this latter character has been proposed as an ecdysozoan autapomorphy, for example by Schmidt-Rhaesa et al. (1998) (see also Jenner, 2002 for a discussion of the coding of this character). The existence of this problem in the matrix of Zrzavý et al. (1998) can be explained by the observation that Z193 uncritically adopted the same character coding and scoring as Hl (Haszprunar, 1996a was used to compile Zrzavý et al.'s data matrix), which was applied to a more restricted set of taxa. Conflict was introduced in Zrzavý et al. (1998) by including the ecdysozoan taxa, which were not considered in Haszprunar (1996a) (see also discussion under Cuticle layers).

Finally, ciliary locomotion of adults is widespread. In addition, there are some problematic scorings such as for Ctenophora (incorrectly scored for muscular locomotion: Hernandez-Nicaise, 1991), and monogonont rotifers (scored ?, but they move mainly by ciliary action: Lorenzen, 1996c), where the scoring of this character is in logical conflict with that of character Z132 which codes for the absence or presence of free locomotion in the adult. Taxa that are scored as lacking free locomotion are at the same time scored as moving by means of muscular action (see also discussion under Locomotion).

Peterson \& Eernisse (2001)

- loss of acrosome as distinct organelle (P20)

- loss of perforatorium (P21)

- loss of prototroch (P48)

- loss of metatroch (P49)

- digestive gut without cilia (P93)

\section{Comments}

While Peterson \& Eernisse (2001) write that their finding of the gnathostomulids and platyhelminths as sister taxa is consistent with the results of $\mathrm{Ax}$ (1995), it should be noted that the analysis of Peterson \& Eernisse (2001) did not include any of the potential plathelminthomorphan synapomorphies proposed by Ax. These studies may thus provide independent evidence for the monophyly of Plathelminthomorpha.

I did not study the scoring of acrosomes or perforatoria (P20, P21) across the Metazoa, but a revised unpublished version of the data matrix of Peterson \& Eernisse (2001) rescored various taxa. Some pertinent remarks can nevertheless be made for the scoring of Plathelminthomorpha, Cycliophora, and Rotifera. P20 scored the gnathostomulids as lacking an acrosome, platyhelminths as possessing one, and the rotifers and Cycliophora as possessing a distinct acrosomal organelle. These designations should be considered as tentative. An acrosome may be present in the tip of the sperm of filospermoid gnathostomulids (Sterrer et al., 1985, fig. 12.4a), and acrosomal vesicles are typically unknown in the platyhelminths, except for some nemertodermatids (Watson, 1999). The presence of a cycliophoran acrosome is unconfirmed (Funch \& Kristensen, 1997; M. V. Sørensen personal communication), and while the Rotifera are solely scored on the basis of Seison, which possesses a distinct acrosome, the other rotifers lack acrosomes.

Moreover, a perforatorium (P21) is defined in the current literature on the basis of electronmicroscopical studies as subacrosomal or periacrosomal 




Fig. 3. Phylogenetic position of the platyhelminths, gnathostomulids, and nemerteans according to (A) Nielsen (1995); (B); Sørensen et al. (2000) (C) Nielsen (2001); (D) Nielsen et al. (1996).

material (Ehlers, 1993; Ax, 1995; note that in older literature the perforatorium may refer to the whole anterior part of the sperm or the entire acrosomal complex). Perforatoria can have different forms, ranging from relatively formless subacrosomal material to taxon specific morphologies including corkand rod-shaped structures (Adiyodi \& Adiyodi, 1983). Data from several taxa such as the polychaetes and echinoderms have shown that this material is likely to be involved in the acrosomal reaction during fertilization (Chia \& Bickell, 1983; Rice, 1992). It appears that wherever an acrosome is developed as a clear organelle or vesicle in the Bilataria, the perforatorium is also present (outside the Bilateria acrosomal vesicles have been reported for cnidarians, but no perforatorium; Ehlers, 1993). This may indicate a logical dependence between well-developed acrosomes and perforatoria for the bilaterians. Consequently P21 and character state 2 of P20 (acrosome as a distinct organelle) may not be independent.

The proposed synapomorphies are predominantly character losses. The significance of the loss of a prototroch and metatroch should be regarded with caution. See under Prototroch and under Metatroch for arguments that would necessitate a rescoring of various taxa for P48 and P49.

Although Micrognathozoa was not included in the analysis, this taxon also completely lacks cilia in the digestive gut, although they are present in 
the pharynx (Kristensen \& Funch, 2000), suggesting that this character may be a synapomorphy of a larger clade including the gnathostomulids. It should further be noted that several phyla are misscored for P93, and that all morphological cladistic analyses support convergent loss of gut cilia in various metazoan clades (see discussion under Intestinal cell ciliation).

\section{(Platyhelminthes Nemertea)}

This clade is known as Parenchymia. Proposed synapomorphies: Nielsen (1995); Nielsen et al. (1996); Nielsen (2001); Sørensen et al. (2000). See figure 3.

Parenchymia is a rather misleading name for the clade of the platyhelminths and nemerteans. It refers primarily to the supposed similarity in acoelomate organization of the body, with parenchymal cells (connective tissue or mesenchymal cells that are neither muscle nor nerve cells) filling the space between body wall and organ systems (Nielsen, 1985). Although parenchymal cells have been observed both in the platyhelminths and the nemerteans, they are thought to have evolved convergently between these phyla and within Platyhelminthes (Rieger, 1985; Turbeville, 1996) (see also discussions under Body cavities). Accordingly, cellular architecture of the body space has not been used in any recent cladistic analysis to unite the parenchymians.

Nielsen (1995); Nielsen (2001)

- larvae with diminutive hyposphere and no ventral nervous system and anus (see NI20)

- adults with only apical nervous system

- no chitin and chitinase

- general shape of larval ciliary bands

\section{Comments}

Although figure 11.4 in Nielsen (1995) reports "diminutive hyposphere," the discussion in chapter 25 makes it clear that a "reduced hyposphere" is meant. This is a phylogenetic assumption not necessarily supported by the phylogenetic analysis as it refers to the evolution of platyhelminth Müller's and Götte's larvae and nemertean pilidium larvae from more typical trochophore larvae (Nielsen, 1985, 1995). For this character as for that concerned with the general shape of the larval ciliary bands, it is important to ascertain that the polyclad larvae and nemertean pilidium larvae are parts of the platyhelminth and nemertean ground patterns, respectively. On the basis of current comparative ontogenetic and phylogenetic data this is very unlikely (see detailed discussions under Larva with strongly reduced hyposphere).

The presumed lack or reduction of the ventral part of the central nervous system is a difficult character and it cannot be scored independently from the supposed sole presence of an apical nervous system. This character is based on the fact that in coelomate spiralians such as the molluscs and annelids, the two components of the central nervous system, i.e., the cerebral ganglion and the ventrally to laterally located longitudinal nerve cords, develop from separate ectodermal anlagen (e.g. Golding, 1992). A similar situation is found in the onychophorans (Eriksson et al., 2003). After the longitudinal nerve cords are formed they secondarily connect to the already formed cerebral ganglia, thus forming the circumoesophageal connectives. However, the platyhelminths and nemerteans do not develop such circumoesophageal connectives. In contrast, their longitudinal nerve cords develop simply as posterior outgrowths from the cerebral (apical) ganglia (e.g. Hartenstein \& Ehlers, 2000; Younossi-Hartenstein \& Hartenstein, 2000), but in general the ontogeny of the platyhelminth and nemertean central nervous systems are not well known (Reuter \& Gustafsson, 1995). However, in contrast to the interpretation of Nielsen $(1995,2001)$, this nervous system configuration may not be restricted to the parenchymians, and may in fact be a plesiomorphy or homoplasy. In contrast to the "higher" molluscs (Ganglioneura or Ganglionata: Cephalopoda, Gastropoda, Bivalvia, Scaphopoda; see Haszprunar, 2000) polyplacophorans presumably develop both the pedal and lateral (pleurovisceral) nerve cords as posterior outgrowths from the cerebral ganglia (Hyman, 1967; Ponder \& Lindberg, 1997 following Hammersten \& Runnström, 1925). Interestingly, available information on the embryol- 
ogy of the aplacophorans (Solenogastres) suggests that at least the lateral (pleural) nerve cords develop as outgrowths from the cerebral ganglion as well (Thompson, 1960; Hyman, 1967). Assuming homology of the tetraneural nervous system across Mollusca, this indicates that the ontogeny of ventral nerve cords is variable. There is then nothing that would dispute homology between the main nerve cords in nemerteans and platyhelminths and molluscs. This might additionally suggest that the separation of the embryonic sources of pre- and posttrochal nervous system elements (cerebral ganglia and main nerve cords, respectively) that is thought to be characteristic of taxa such as the molluscs and the annelids (e.g. Dorresteijn et al., 1993; Raineri, 2000), may have evolved convergently.

I also fail to perceive the fundamental difference emphasized by Nielsen $(1994,1995,2001)$ between the parenchymian nervous system and the nervous system of the remaining protostomes in the absence or presence of a circumesophageal nervous concentration or brain, respectively. Rhabditophoran platyhelminths, nemerteans and many other protostome phyla share the possession of an anterodorsally located cerebral ganglion, which is connected to the ventral part of the central nervous system by connectives. Apart from the variable position of the mouth this construction appears very similar. Thus the adult nervous system of taxa such as the aplacophoran and polyplacophoran molluscs, onychophorans, various polychaetes, and nemerteans all localize the cerebral ganglia principally anterodorsal to the digestive canal, with cord-like connectives running caudad through the body (Scheltema et al., 1994; Eernisse \& Reynolds, 1994; Westheide, 1996; Eriksson \& Budd, 2000). In other taxa, the anterior nervous system may become more ganglionated resulting in the development of brain-like (ganglionate) circumesophageal connectives and subesophageal ganglia, e.g., in the higher molluscs (a clade composed of the Conchifera minus Monoplacophora is sometimes termed Ganglioneura or Ganglionata to reflect the evolution of ganglia), several polychaetes, tardigrades and arthropods (Westheide, 1996; Dewel \& Dewel, 1996; Dewel et al., 1999).

However, the current data is unequivocal. Reliable comparative embryological studies are scanty, frequently old, and accounts may be contradictory, e.g., Kowalevsky (1882) vs. Hammarsten \& Runnström (1925) for the ontogeny of the polyplacophoran nervous system. In addition, the uncertain phylogenetic position of the acoelomorphs, and the morphological variation in central nervous systems in taxa such as the molluscs and annelids, makes it very diffcult to infer primitive character states. Furthermore, recent studies indicate the sensitivity of the results to variation in analytical techniques, such as specificity of the neural markers employed for visualization of the nervous system (see Raineri, 2000). Furthermore, different pictures of nervous system morphology and neurogenesis may emerge when different species are used, or different developmental stages are studied (Friedrich et al., 2002) Finally, it is difficult to trace the origin of the elements of the adult nervous system in the earliest ontogenetic stages in taxa such as the molluscs and annelids, where larval and adult nervous systems may be remarkably independent (Lacalli, 1984; Golding, 1992; Marois \& Carew, 1997; Dickinson et al., 2000).

The lack of an anus in polyclad and pilidium larvae might be a unique parenchymian autapomorphy, but the validity of this character is again dependent upon the assumption that these larvae are included in the ground patterns of platyhelminths and nemerteans. As argued elsewhere in this paper, current information does not support this possibility. The same holds true for the character that deals with the general form of the larval ciliary bands. In contrast, lack of an adult anus has been considered a plathelminthomorphan apomorphy.

The lack of the ability to synthesize chitin may be a synapomorphy (albeit not unique) of the platyhelminths and nemerteans.

Nielsen et al. (1996)

- larvae or adult with downstream-collecting ciliary bands of compound cilia on multiciliate cells (N22)

\section{Comments}

N22 is highly homoplastic and is dependent on the unsupported premise that planktotrophic polyclad 


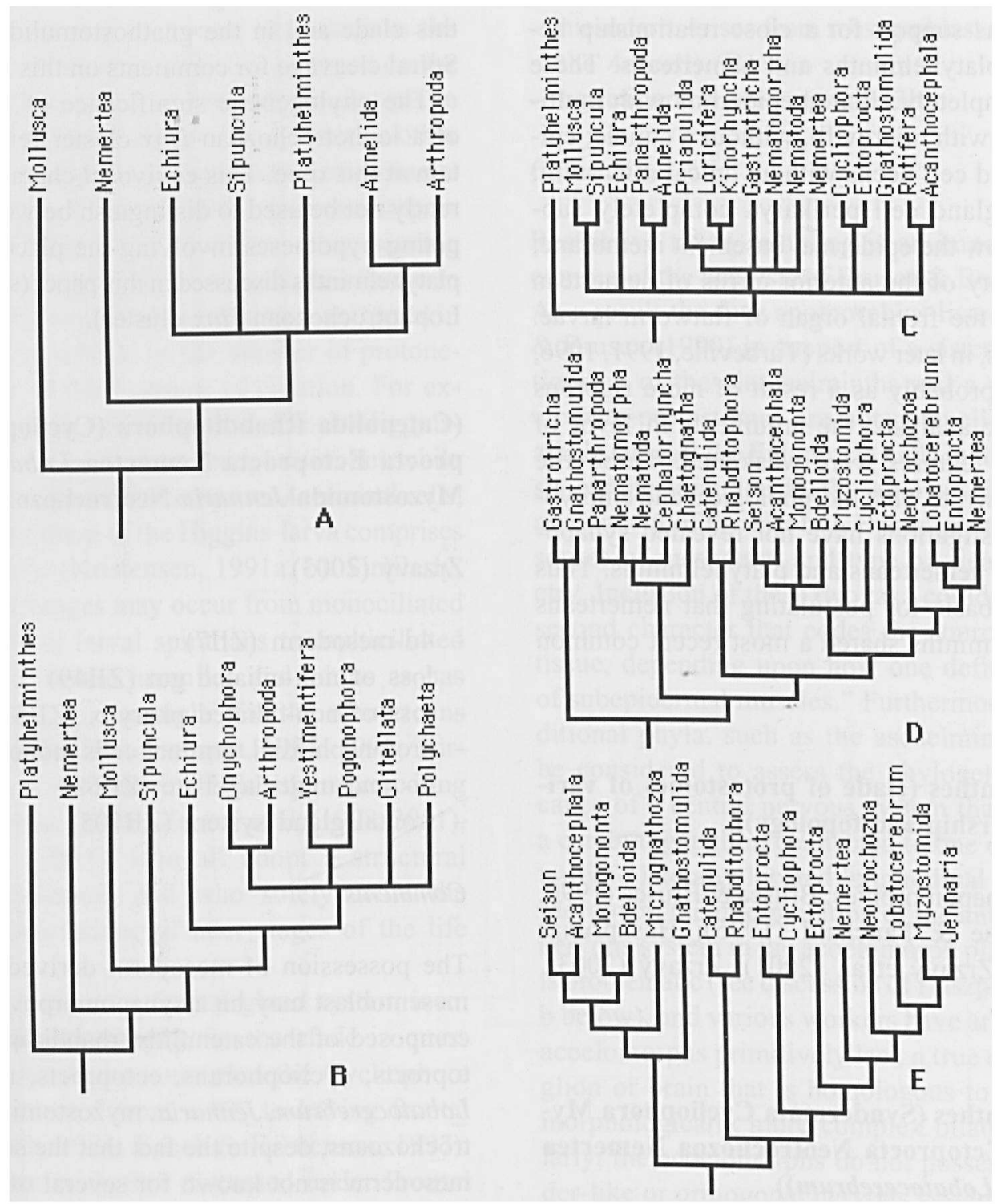

Fig. 4. Phylogenetic position of the platyhelminths, gnathostomulids, and nemerteans according to (A) Brusca \& Brusca (1990); (B) Rouse \& Fauchald (1995); (C) Brusca \& Brusca (2003); (D) morphological analysis of Zrzavý et al. (2001); (E) morphological analysis of Zrzavý (2003).

and pilidium larvae are plesiomorphic features for the platyhelminths and nemerteans respectively (see relevant comments under Larva with strongly reduced hyposphere). Moreover, although polyclad Müller's or Götte's larvae possess bands of pronounced cilia, these are not compound, and their function in planktotrophy remains unattested (Nielsen, 1987, 1995, 1998a). Downstream ciliary feeding is equally undocumented for nemertean pilidium larvae.
Sørensen et al. (2000)

- larva with strongly reduced hyposphere (S28)

\section{Comments}

This is a very weak character (see discussion under Larva with strongly reduced hyposphere).

" Although not within the context of a cladistic analysis, Turbeville \& Ruppert (1985) (followed by Bogitsh \& Harrison, 1991) interpreted some 
characters as support for a close relationship between the platyhelminths and nemerteans. These include completely ciliated epidermis with multiciliate cells with microvilli, without a cuticle; epidermal gland cell necks without apical microvilli; epidermal gland cell perikarya completely submerged below the epidermal basement membrane; and similarity of the anterior cirrus of nemertean larvae with the frontal organ of flatworm larvae. Nevertheless, in later works (Turbeville, 1991, 1996, 2002), and probably as a result of more rigorous phylogenetic analysis, the arguments in favor of using these features were weakened. Turbeville (1991: 326) wrote "that morphological and embryological investigations have not revealed synapomorphies of nemerteans and platyhelminths. Thus there is no basis for postulating that nemerteans and platyhelminths shared a most recent common ancestor."

\section{(Platyhelminthes (clade of protostomes of vari- able membership and topology))}

Proposed synapomorphies: Brusca \& Brusca (1990, 2003); Rouse \& Fauchald (1995); Haszprunar (1996a, b); Zrzavý et al. (2001); Zrzavý (2003). See figure 4.

(Platyhelminthes (Syndermata Cycliophora Myzostomida Ectoprocta Neotrochozoa Nemertea Entoprocta Lobatocerebrum))

Zrzavý et al. (2001)

- spiral quartet cleavage (ZI1)

- lophotrochozoan complement of Hox genes (ZI59)

\section{Comments}

Platyhelminthes in Zrzavý et al. (2001) represent Rhabditophora and Catenulida. The presence of spiral quartet cleavage is a proper putative synapomorphy for the platyhelminths and a clade of other protostomes. However, this would suggest convergent evolution of spiral quartet cleavage in this clade and in the gnathostomulids (see under Spiral cleavage for comments on this implication).

The phylogenetic significance of the presence of a lophotrochozoan Hox cluster remains uncertain at this time. This equivocal character can currently not be used to distinguish between the competing hypotheses involving the placement of the platyhelminths discussed in this paper (see also under Lophotrochozoan Hox cluster).

(Catenulida Rhabditophora (Cycliophora Entoprocta Ectoprocta Nemertea Lobatocerebrum Myzostomida Jennaria Neotrochozoa))

\section{Zrzavý (2003)}

- 4d-mesoderm (ZII7)

- loss of non-ciliated gut (ZII49)

- loss of non-ciliated pharynx (ZII51)

- protonephridial terminal cells monoflagellate $\rightarrow$ bi- and multiflagellate (ZII68)

- frontal gland system (ZII105)

\section{Comments}

The possession of mesoderm derived from a $4 \mathrm{~d}$ mesentoblast may be a synapomorphy for a clade composed of the catenulids, rhabditophorans, entoprocts, cycliophorans, ectoprocts, nemerteans, Lobatocerebrum, Jennaria, myzostomids, and neotrochozoans, despite the fact that the source of the mesoderm is not known for several of these taxa. The evolution of ciliated intestinal cells may be a convergent synapomorphy of the clade that includes the non-acoelomorph platyhelminths in Zrzavý (2003). The nemertodermatids appear to lack ciliated intestinal cells primitively, in contrast to the scoring of a "?" for ZII49 (Lundin \& Sterrer, 2001).

The possession of ciliated pharynges is a potential synapomorphy of the clade in question, however, the optimization of this character is ambiguous.

The possession of protonephridial terminal cells with more than one cilium is a proper potential synapomorphy of the clade under consideration. 
However, the scoring of certain taxa, such as the annelids and nemerteans, could be changed on the basis of a consideration of ontogenetic changes in protonephridial morphology. As with many other characters, protonephridial organization may change during ontogeny. Consequently, it becomes important to justify semaphoront choice when scoring protonephridial characters. Ontogenetic change in protonephridial organization may be manifested in different ways, such as in the number of protonephridial cells or their pattern of ciliation. For example, both adult loriciferans and their Higginslarvae possess a pair of protonephridia, but while the adult protonephridium comprises 4 terminal cells, the protonephridium of the Higgins-larva comprises 7 terminal cells (Kristensen, 1991a, b). Similarly, ontogenetic changes may occur from monociliated terminal cells in larval spiralians to multiciliated terminal cells in the juveniles and adults as has been observed, for example, for the nemerteans and the annelids (Bartolomaeus, 1985, 1995; Turbeville, $1991 \&$ refs. therein). This requires rescoring in Sørensen et al. (2000), Zrzavý et al. (1998, 2001), and Zrzavý (2003), who all adopt a structural homology criterion, and who solely score the situation characteristic of later stages of the life cycle.

The possession of a frontal gland system is an ambiguous synapomorphy for the clade in question, but it should be noted that the Cycliophora should be rescored from a "?" to having frontal glands (Funch, 1996; Funch \& Kristensen, 1997). This character is further discussed under Frontal gland complex.

\section{(Platyhelminthes (Nemertea Sipuncula Echiura Mollusca Annelida Pogonophora Arthropoda))}

\section{Brusca \& Brusca (1990)}

- cerebral ganglion issues pairs of longitudinal cords connected by transverse commissures (ladder-like), with tendency to emphasize ventral or ventrolateral cords (reduced to a single ventral cord in some taxa) (B64c)

- sheets of subepidermal muscles derived, at least in part, from $4 \mathrm{~d}$ mesoderm (B58)
- mesoderm arises from mesentoblast (primitively the $4 \mathrm{~d}$ cell) (B18)

- typical spiral cleavage (B13b)

\section{Comments}

Restrictive taxon sampling is an important shortcoming of the analysis of Brusca \& Brusca (1990). As a result the four apomorphies listed by Brusca $\&$ Brusca (1990) in support of a sister group relationship of the platyhelminths and a clade of coelomate protostomes are not compelling. For example, at least the Entoprocta and Gnathostomulida should be included in the analysis for a proper assessment of the last two characters that code the source of mesoderm and type of cleavage geometry. Inclusion of these two taxa could also test the second character that codes the source of muscle tissue, depending upon how one defines a "sheet of subepidermal muscles." Furthermore, many additional phyla, such as the aschelminths, have to be considered to assess the phylogenetic significance of a central nervous system that consists of a cerebral ganglion connected to one or more ventrally to laterally located longitudinal nerve cords. Moreover, the interpretation of the anterior central nervous system in the acoelomorph platyhelminths is problematic (see discussion of Haszprunar, 1996a, $\mathrm{b}$ below), and various workers have argued that the acoelomorphs primitively lack a true cerebral ganglion or brain that is homologous to the brain of morphologically more complex bilaterians. Similarly, the acoelomorphs do not possess a true ladder-like or orthogonal nervous system, and the interpretation of this feature in other phyla may be problematic, e.g., molluscs. The significance of the acoelomorphs for the reconstruction of the platyhelminth ground pattern should also be understood when scoring typical spiral cleavage for the platyhelminths. The acoelomorphs (published information is so far restricted to acoels) exhibit a unique duet cleavage geometry that may or may not be derived from typical spiral quartet cleavage (Henry et al., 2000). See below for complete discussions of spiral cleavage, cerebral ganglia, and mesentoblast derived mesoderm. 

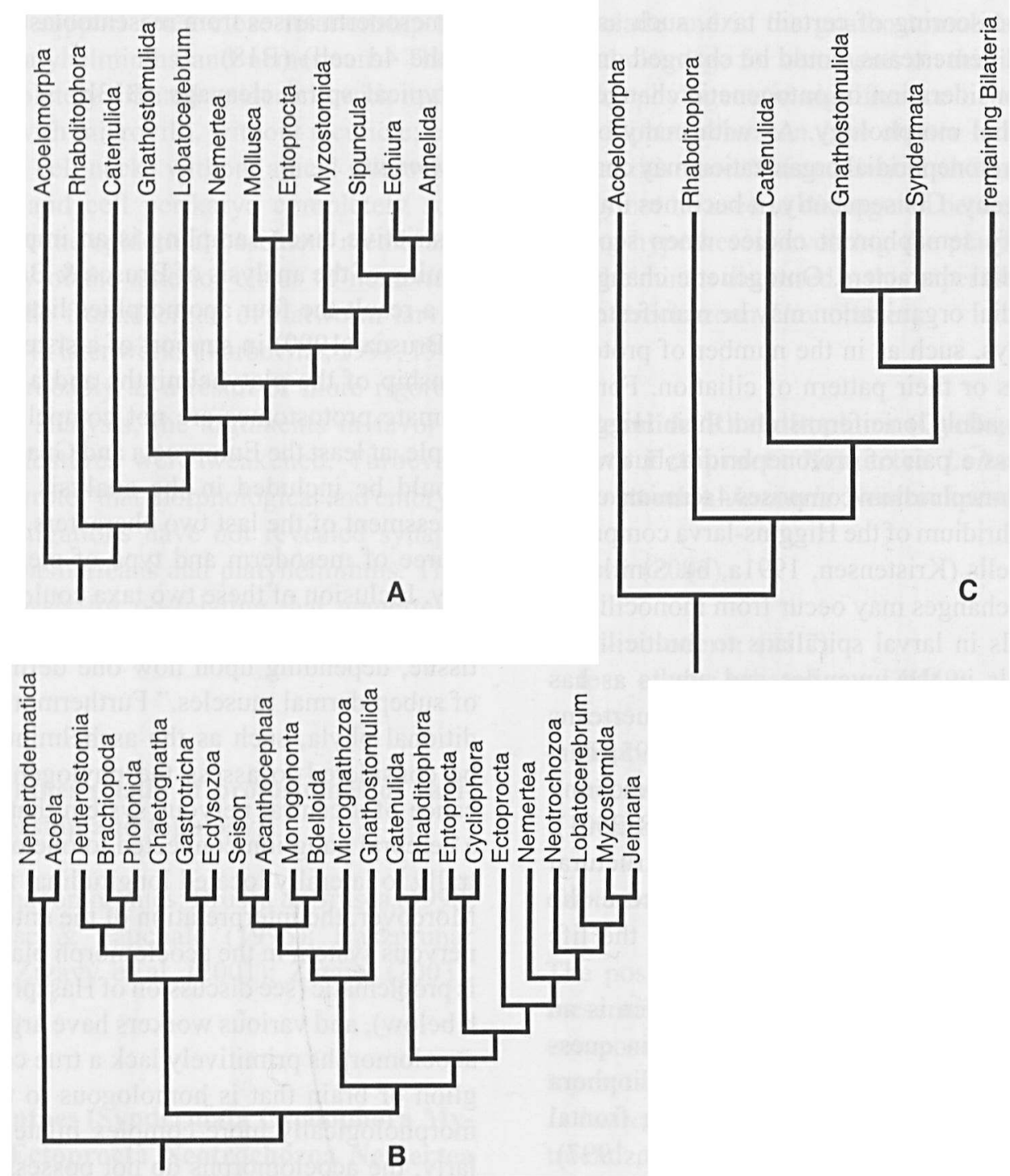

Fig. 5. Phylogenetic position of the platyhelminths, gnathostomulids and nemerteans according to (A) Haszprunar (1996a); (B) morphological analysis of Zrzavý (2003); (C) Haszprunar (1996b).

\section{(Platyhelminthes (Cycloneuralia Euspiralia Gnathifera Cycliophora))}

Brusca \& Brusca (2003)

- cleavage pattern fundamentally spiral (BI18)

- synaptic nervous system concentrated ventrally or ventrolaterally (BI52)

- entomesoderm derives from a single (mesentoblast) cell, typically the $4 \mathrm{~d}$ cell (BI74)

- sheets of subepidermal muscle derived at least in part from $4 \mathrm{~d}$ mesoderm (BI75)

\section{Comments}

Spiral cleavage may be a proper potential synapomorphy that unites the platyhelminths with a clade of other protostomes, but it is doubtful whether spiral cleavage can serve to distinguish all the protostomes (except the chaetognaths) from the deuterostomes and lophophorates as is suggested by the cladogram in Brusca \& Brusca (2003). Several scoring mistakes have to be corrected before the true phylogenetic significance of spiral cleavage can become apparent. BI18. incorrectly scored spiral cleavage 
as being possessed by the rotifers, acanthocephalans, onychophorans, tardigrades, and arthropods (see discussion under Spiral cleavage). Rescoring of BI18 for these taxa may help resolve the protostome polytomy of Brusca \& Brusca (2003).

The presence of a ventral nervous system is generally considered as one of the most reliable characteristics of the protostomes (Nielsen, 1994, 2001), as is reflected in the alternative name Gastroneuralia (the protostomes minus the lophophorates). Consequently, the possession of a ventral or ventrolateral nervous system appears to be a proper synapomorphy of the platyhelminths and the other gastroneuralians. However, certain striking differences in ventral nervous system morphology have nevertheless evolved within the gastroneuralians. Brusca \& Brusca (2003) code this diversity by erecting a separate and autapomorphic character state for the chaetognath nervous system. Yet, the entoprocts are simply scored the same as the other gastroneuralians, despite the fact that their ventral nervous system departs even more than the chaetognath nervous system from the longitudinal nerve cords that characterize most gastroneuralians. See also discussion under Orthogonal nervous system.

The derivation of the endomesoderm from the $4 \mathrm{~d}$ mesentoblast has been reported for phyla with spiral cleavage such as the entoprocts, platyhelminths, nemerteans, and the neotrochozoans. However, in contrast to the scoring for $\mathrm{BI} 74$, the $4 \mathrm{~d}$ mesentoblast has not been documented as the source of mesoderm in the panarthropod phyla (see discussion under Spiral cleavage). The same conclusion pertains to the scoring of the panarthropods for BI75.

(Platyhelminthes (Nemertea Mollusca Sipuncula Echiura Annelida Pogonophora Arthropoda Onychophora))

Rouse \& Fauchald (1995)

- no synapomorphies

\section{Comments}

The cladistic analysis of Rouse \& Fauchald (1995) yields a similar placement of platyhelminths as found by Brusca \& Brusca (1990), but the work of the former does not constitute independent support for the phylogenetic position of the platyhelminths because synapomorphies are lacking. The choice of the platyhelminths as an out-group led to a default position at the base of the cladogram of Rouse \& Fauchald (1995).

\section{Platyhelminthes as a basal bilaterian grade} Proposed synapomorphies: Haszprunar (1996a, b); Zrzavý (2003). See figure 5.

(Acoelomorpha (Rhabditophora (Catenulida Gnathostomulida Nemertea Lobatocerebrum Kamptozoa Mollusca Myzostomida Sipuncula Echiura Annelida)))

Haszprunar (1996a, b)

\section{Comments}

A number of studies subdivided Platyhelminthes into various different subgroups to test for the monophyly of the phylum: Eernisse et al. (1992): Acoelomorpha, Rhabditophora; Zrzavý et al. (1998), Littlewood et al. (1999a), Giribet et al. (2000), and Zrzavý (2003): Acoela, Nemertodermatida, Catenulida, various rhabditophoran subtaxa; Haszprunar (1996a, b): Acoelomorpha, Rhabditophora, Catenulida; Peterson \& Eernisse (2001): Acoela, Nemertodermatida, Catenulida, Rhabditophora; Zrzavý et al. (2001): Catenulida, Rhabditophora. Of these studies Haszprunar (1996a, b) found morphological support for the paraphyly of Platyhelminthes at the base of the Bilateria, with the acoelomorphs branching of first, followed by the rhabditophorans, and finally the catenulids as the closest relatives to the remaining bilaterians. The placement of the acoelomorphs as the earliest branching living bilaterians is of considerable interest because it would bridge "significantly the large differences between the diplo- and triploblastic level of organization" (Haszprunar, 1996b: 45). The apparent molecular support for this hypothesis (Ruiz-Trillo et al., 1999, 2002; Baguñà et al., 2001; Jondelius et al., 2002; Telford et al., in press; but see Littlewood et al., 1999a, Giribet et al., 2000 and Peterson \& Eernisse, 
2001 for critical comments on the molecular "support" for platyhelminth paraphyly) makes this hypothesis especially worthy of consideration. These results have already lead several zoologists to reexplore the heuristic value of the acoel level of organization as a bridge between the non-bilaterian and bilaterian body architectures (see Rieger \& Ladurner, 2001; Baguñà et al., 2001; see Hyman, 1940, 1959, and Salvini-Plawen, 1978 for a discussion of kindred views from a generation past).

Most critical for Haszprunar's hypothesis are those characters that set the Acoelomorpha apart from the remaining bilaterians, i.e., the synapomorphies supporting the monophyly of the sister clade of Acoelomorpha. These synapomorphies are:

- septate junctions (H6)

- cerebral ganglion (H13; Halla)

- orthogonal nervous system (H14)

- protonephridia (H20; HallIb)

- spiral quartet cleavage (H33)

- fixed cell fate during cleavage (H35)

Various molecular phylogenetic analyses that position the acoelomorphs at the base of the bilaterians cited several of these morphological traits in support of their conclusions (Ruiz-Trillo et al., 1999; Baguñà et al., 2001; Jondelius et al., 2002; Telford et al., in press). However, we must ask how convincing are these synapomorphies for uniting all bilaterians to the exclusion of the acoelomorphs?

Haszprunar (1996a) created a special character state to code for the supposedly unique ultrastructure of acoel septate junctions, which are of the anastomosing type also found in the echinoderms and hemichordates (Green \& Bergquist, 1982). It is relatively easy to see a plethora of unique types of septate junctions across the Metazoa, which are characteristic for more or less inclusive clades (Green \& Bergquist, 1982). It is simply a matter of emphasizing the unique features of the acoelomorph septate junctions over their similarities with other types of invertebrate septate junctions that allows the creation of a special character state, but that does not negate the fundamental homology of different types of septate junctions.

Haszprunar (1996a, b) argued that the plesiomorphic state for the acoelomorphs is the presence of a simple neural plexus that is comparable to that found in the cnidarians and ctenophores. This implies that the acoelomorphs primitively lack a cerebral ganglion, a conclusion reasonably supported by available information. However, several higher acoelomorphs have independently evolved anterior neural concentrations ('brains'). This is in agreement with the most recent interpretations of the acoelomorph central nervous system (Raikova et al., 1998, 2001). Raikova et al. (1998: 76) conclude that there appears to be a "deep gap [in nervous system organization] between the Acoela and the other Platyhelminths." Nevertheless, Raikova et al. (2001) also point out that further research is necessary to reach any definitive conclusions about the phylogenetic significance of acoelomorph neuroanatomy.

- The unique structure of the acoelomorph nervous system is also reflected in the organization of the nerve 'cords.' The acoelomorphs do not have an orthogonal nervous system with a pair of major longitudinal nerve cords that are regularly connected by transverse commissures (Rieger et al., 1991b; Raikova et al., 2001). It is therefore not surprising to find that recent studies on the origin and evolution of an orthogonal nervous system organization hardly mention acoelomorphs at all (Reuter \& Gustafsson, 1995; Reuter et al., 1998).

The lack of protonephridia seconds a basal position of the acoelomorphs in the metazoan phylogeny, implying that the acoelomorphs primitively lack protonephridia. The widespread distribution of nephridial systems within the remaining bilaterians led Jondelius et al. (2002) to propose the hame Nephrozoa for all Bilateria excluding the acoels and nemertodermatids. Interestingly, all cladistic studies considered in this paper, with the exception of Haszprunar (1996a, b), suggest that protonephridia are homoplastic within Bilateria, and have evolved not at the base, but within the Bilateria, usually at the base of a large protostome clade. In these analyses multiple losses of protonephridia are indicated for taxa such as Panarthropoda, and Nematoida (Nielsen et al., 1996; Nielsen, 2001; Sørensen et al., 2000; Zrzavý et al., 1998, 2001; Zrzavý, 2003). However, the evolution of excretory systems may be corroborated as a synapomorphy of the nephrozoans by mounting evidence for a structural and 
Table 1. Comparison of the treatment of potential evidence for platyhelminth paraphyly suggested by Haszprunar (1996a, b) in different cladistic analyses. The listed characters are diagnostic synapomorphies for a monophyletic clade of all bilaterians exclusive of acoelomorphs as suggested by the cladistic analysis of Haszprunar (1996a).

\begin{tabular}{|c|c|c|c|}
\hline Haszprunar (1996a) & Zrzavý et al. (1998) & Peterson \& Eernisse (2001) & Littlewood et al. (1999a) \\
\hline $\begin{array}{l}\text { acoelomorph type } \\
\text { septate junctions (H6) }\end{array}$ & $\begin{array}{l}\text { no separate character } \\
\text { state for acoelomorphs }(\mathrm{Z} 179)\end{array}$ & $\begin{array}{l}\text { no separate character state for } \\
\text { acoelomorphs (P3) }\end{array}$ & not included in analysis \\
\hline cerebral ganglion (H13) & ok $(\mathrm{Z} 237)$ & not included in analysis & $\begin{array}{l}\text { combined with orthogonal nervous } \\
\text { system (L55) and scoring assumes } \\
\text { evolutionary polarity for } \\
\text { acoelomorphs }\end{array}$ \\
\hline $\begin{array}{l}\text { orthogonal nervous } \\
\text { system }(\mathrm{H} 14)\end{array}$ & ok (Z245) & not included in analysis & $\begin{array}{l}\text { combined with cerebral ganglion } \\
\text { (L55) and scoring assumes } \\
\text { evolutionary polarity for } \\
\text { acoelomorphs }\end{array}$ \\
\hline protonephridia $(\mathrm{H} 20)$ & ok $(\mathrm{Z} 48)$ & not included in analysis & $\begin{array}{l}\text { Scoring assumes evolutionary } \\
\text { polarity for acoelomorphs (L56) }\end{array}$ \\
\hline spiral quartet cleavage (H33) & ok (Z7) & ok (P29) & $\begin{array}{l}\text { Scoring assumes evolutionary } \\
\text { polarity for acoelomorphs (L52) }\end{array}$ \\
\hline $\begin{array}{l}\text { fixed cell fate during cleavage } \\
\text { (H35) }\end{array}$ & ok (Z9) & not included in analysis & not included in analysis \\
\hline
\end{tabular}

functional continuum of cell types variously part of protonephridial and metanephridial systems across the Bilateria (see discussion under Podocytes/terminal cells/nephrocytes).

The duet spiral cleavage of the acoels (and purportedly also of the nemertodermatids: Nielsen, 2001) has often been considered as secondarily derived from spiral quartet cleavage, but this interpretation has obviously been influenced by the heretofore widely accepted position of the acoelomorphs within the Platyhelminthes (see under Spiral cleavage). All comprehensive morphological cladistic analyses suggest that spiral quartet cleavage has evolved at the base of a clade Spiralia, not at the base of the Bilateria.

New information on early acoel development (Henry et al., 2000) clearly contradicts the scoring of H35 (absence of fixed cell fate during cleavage). Although it may be concluded that the acoels possess what is for spiralians a remarkable capacity for regulative development in early ontogeny, it is not true that their cell fate determination during early development has more in common with cnidarian embryology than with the embryology of all other metazoan phyla, as is implied by the scoring of $\mathrm{H} 35$.

As was noted before, various cladistic analyses (Zrzavý et al., 1998; Littlewood et al., 1999a; Giribet et al., 2000, relying on Zrzavý et al.'s data matrix; Peterson \& Eernisse, 2001; Zrzavý, 2003) that were published subsequent to Haszprunar's (1996a) study, split up the platyhelminths sufficiently to allow a cladistic test of Haszprunar's hypothesis of platyhelminthan paraphyly at the base of the Bilateria. I will here explore whether these newer analyses can be considered as effective tests of Haszprunar's proposal.

The comprehensive taxon sampling in Zrzavý et al. (1998), Peterson \& Eernisse (2001), and Zrzavý (2003) in principle allowed a test of the position of the acoelomorphs at the base of the Bilateria to be tested. However, the first two studies supported a position of the acoelomorphs deeply within the Bilateria, while Zrzavý (2003) confirmed the position of the acoelomorphs basal to the other bilaterians. However, these analyses differed with respect to testing efficacy. The analysis of Peterson \& Eernisse (2001) did not include all characters that could serve to separate the acoelomorphs from the other platyhelminths and bilaterians (see Table 1). Therefore, their study was not a rigorous test of Haszprunar's hypothesis.

Peterson \& Eernisse (2001) identified the different positions of the acoelomorph platyhelminths in their morphological and molecular analyses (deep within, and at the base of the Bilateria, respectively) 
as an indication of major conflict between these data sources. However, in view of their restrictive sampling of morphological characters, this apparent conflict should be reassessed.

In contrast, the analysis by Zrzavý et al. (1998) included all characters that Haszprunar (1996a) suggested as supporting a basal position of the acoelomorphs. However, the coding and scoring of these characters were not necessarily the same, but this reflects justifiable differences in character interpretation (see Table 1). Therefore, this analysis constitutes a test of Haszprunar's hypothesis of platyhelminth paraphyly at the base of the Bilateria, and it is important that they did not confirm Haszprunar's results.

The analysis of Zrzavý (2003) included most of the characters that separated the acoelomorphs from the remaining bilaterians in Haszprunar's (1996a) study, with the exception of characters coding for septate junctions, and cell fate determination during early embryogenesis. Of these included characters, Zrzavý (2003) supported the presence of a cerebral ganglion (ZII92) and an orthogonal nervous system (ZII97) as synapomorphies of the Bilateria to the exclusion of the acoelomorphs. These features are discussed under Cerebral ganglion, brain, and Orthogonal nervous system, respectively. In contrast, spiral quartet cleavage (ZII2) and protonephridia (ZII66) were optimized as synapomorphies that evolved within the Bilateria in the analysis of Zrzavý (2003) (see discussion under Spiral cleavage and Protonephridia, respectively). In addition, Zrzavý (2003) identified several novel synapomorphies of the Bilateria to the exclusion of the acoelomorphs, viz., the presence of a hindgut and anus (ZII50), and sacular gonads (ZII89). These latter two features are discussed under Anus and Gonads asacular or sacular, respectively.

Littlewood et al. (1999a) did not attempt to test the position of the acoelomorphs at the base of the Bilateria, but their subdivision of Platyhelminthes into several subtaxa did allow a test of platyhelminth monophyly. However, the adopted character selection and character coding and scoring in Littlewood et al. (1999a) clearly indicate that their results were biased towards finding support for platyhelminth monophyly (see Table 1). First, the characters on cell fate and septate junctions were not included in their analysis, removing two of the six characters that could conceivably support platyhelminth paraphyly (but see comments above for a reinterpretation of these features). Second, the combination of two characters into a single character involving the presence of a cerebral ganglion and an orthogonal nervous system further removed one more character that could buttress platyhelminth paraphyly. Third, the scoring of the included characters favored the grouping of the acoelomorphs with the other platyhelminths by a priori assuming that the morphology of the acoelomorph nervous system, their lack of protonephridia, and the nature of acoel embryology could well be modifications of the 'normal' platyhelminth condition (acoelomorphs were scored as having cleavage homologous to spiral quartet cleavage, and '?' for the other two characters). We can thus conclude that Littlewood et al. (1999a) effectively removed any testing power for the hypothesis of platyhelminth paraphyly.

In conclusion, Haszprunar's (1996a, b) hypothesis for a paraphyletic Platyhelminthes at the base of the Bilateria is not supported by most comprehensive analyses, with the exception of Zrzavý (2003). This is perhaps not surprising since the taxon sampling of Haszprunar (1996a) was restricted to a selection of protostome taxa only. Consequently, his conclusion that acoelomorphs are basal bilaterians, rather than protostomes, is unsupported by his own data.

\section{Placing Platyhelminthes: comparison of alter- Inative hypotheses}

With the exception of Haszprunar's (1996a, b) analyses, the platyhelminths are universally placed within, not at the base of, the (protostomian) bilaterians on the basis of all other recent morphological cladistic analyses. These analyses have identified four potential sister groups of Platyhelminthes: Gnathifera, Gnathostomulida (Plathelminthomorpha hypothesis), Nemertea (Parenchymia hypothesis), and a larger assemblage of bilaterians that may either include coelomate spiralians only, or a larger clade of non-coelomate and coelomate bilaterians. Guided by the criteria listed at the beginning of this paper, 
is it possible to decide which of these hypotheses is the most probable?

With the exception of just two characters (lack of mitosis in somatic cells, lack of anus), none of the characters advanced in support of a sister group relationship of the platyhelminths with either the gnathiferans by Ahlrichs (1995) and Melone et al. (1998), or the gnathostomulids by Ax (1985, 1989, 1995) and Eernisse et al. (1992), fulfilled the domain of definition. These characters may thus represent symplesiomorphies or homoplasies. Similarly, none of the synapomorphies proposed by Brusca \& Brusca (1990) in support of a clade of platyhelminths and coelomate spiralians satisfied the domain of definition. The range of included taxa in these studies was too limited for a proper evaluation of the phylogenetic significance of the relevant characters.

The placement of the platyhelminths in the analyses of Nielsen et al. (1996) and Rouse \& Fauchald (1995) is biased by restrictive taxon sampling. Nielsen (1995) argued that the gnathostomulids were most likely derived polychaetes. The resulting exclusion of the gnathostomulids from the analysis of Nielsen et al. (1996) therefore did not allow support for a plathelminthomorphan clade to be tested. The placement of the platyhelminths as a sister group to a clade of coelomate spiralians in Rouse \& Fauchald (1995) is not supported by synapomorphies, but instead is a default result given the choice of platyhelminths as an out-group.

Differences in character selection contribute substantially to the existence of conflicting hypotheses for the phylogenetic placement of platyhelminths. For example, with the exception of a few characters (compact acrosome in sperm, protonephridia) neither Nielsen et al. (1996) nor Sørensen et al. (2000) included any of the characters found in support of Plathelminthomorpha in the other studies. Nielsen (2001) excluded the character on sperm acrosomes from his new data matrix without explicit comment. Furthermore, with the exception of a character on chitin synthesis, which is also included in the matrix of Zrzavý et al. (1998) (Z172), the characters advanced in support of the monophyly of Parenchymia by Nielsen (1995), Nielsen et al. (1996), and Sørensen et al. (2000) are unique to these studies. Interestingly, while the morphological matrix of Zrzavý et al. (1998) represents the most encompassing phylogenetic data set for the Metazoa compiled to date, it neither included nor discussed all characters previously suggested to be relevant for placing the platyhelminths, including mode of sperm transfer, mode of fertilization, larval similarities between platyhelminths and nemerteans, and presence of an accessory centriole in sperm. It should also be noted that some characters that have been proposed in support of a particular hypothesis are actually compatible with other, sometimes even all, advanced alternatives. For example, the presence of protonephridia was suggested as a plathelminthomorphan autapomorphy by the analysis of Meglitsch \& Schram (1991) (M31). However, the presence of protonephridia cannot be used to argue against the unison of the platyhelminths with gnathiferans, nemerteans, or a larger clade of spiralians, because protonephridia are uniformly present in all these taxa. A similar argumient can be made for other characters, such as possession of a lophotrochozoan complement of Hox genes, lack of prototroch and metatroch, and spiral quartet cleavage (compatible with all but Gnathifera as a platyhelminth sister group).

Two of the characters proposed in support of a monophyletic Plathelminthomorpha suffer from problematic character coding: the character on direct internal fertilization (E119) inappropriately combines mode of sperm transfer and mode of fertilization; the character on cuticle layers (Z193) should be adjusted for taxa exhibiting more than two distinct layers.

Four studies in particular suffer from lapses in character scoring, namely Meglitsch \& Schram (1991), Eernisse et al. (1992), Zrzavý et al. (1998), and Brusca \& Brusca (2003). One of the three characters in support of a monophyletic Plathelminthomorpha in Meglitsch \& Schram (1991) (M6) was incorrectly scored for both platyhelminths and gnathostomulids. Three of the four synapomorphies in Eernisse et al. (1992), and eight of the 11 synapomorphies in Zrzavý et al. (1998) proposed in support of a clade Plathelminthomorpha contained problematic and incorrect scorings for several taxa (Table 2). In addition, the scoring of Z258 (coding for mode of adult locomotion) is in logical conflict with Z132 
Table 2. Summary of alternative sister taxa for Platyhelminthes with diagnostic synapomorphies and comments. See text for discussion.

\begin{tabular}{|c|c|}
\hline Sister taxon & Synapomorphies and source \\
\hline Gnathifera & $\begin{array}{l}\text { internal sperm deposition (A13) } \\
\text { internal fertilization (A13) } \\
\text { no mitosis in somatic cells (A13) }\end{array}$ \\
\hline & $\begin{array}{l}\text { filiform sperm (A13) } \\
\text { lack of accessory centriole in sperm (A13) } \\
\text { statocysts (Me60) }\end{array}$ \\
\hline
\end{tabular}
statocysts (Me60)

\section{Gnathostomulida}

direct sperm transfer $(\mathrm{Ax}, 1985,1989,1995)$

internal fertilization (Ax, 1985, 1989, 1995)

filiform sperm (Ax, 1985, 1989, 1995; E118; Z117)

no mitosis in somatic cells (Ax, 1995)

hermaphroditism (Ax, 1985, 1989, 1995; E117; Z126)

lack of anus (M16, Z72)

no special muscle cells (M6)

protonephridia (M31)

lack of anus with proctodeum (E96)

digestive gut without cilia (P93)

direct internal fertilization (E119)

absence of epidermal mitosis (Z191)

lack of coeloblastula (Z11)

sacular $\rightarrow$ asacular gonads $(\mathrm{Z} 110)$

monoflagellate $\rightarrow$ biflagellate sperm (Z115)

lack of compact acrosome (Z120; P20)

loss of perforatorium (P21)

rare/absent $\rightarrow$ dominant asexual reproduction $(\mathrm{Z} 128)$

two layered $\rightarrow$ simple cuticle (Z193)

adult locomotory muscular $\rightarrow$ ciliary (Z258)

loss of prototroch (P48)

loss of metatroch (P49)

\section{Nemertea}

larvae with diminutive hyposphere, no ventral nervous system and anus (Nielsen, 1995, 2001) adults with apical nervous system only (Nielsen, 1995, 2001)

no chitin and chitinase (Nielsen, 1995, 2001)

\section{Comments}

domain of definition not fulfilled

domain of definition not fulfilled

may be homoplastic or symplesiomorphic (eutelic

taxa)

domain of definition not fulfilled

domain of definition not fulfilled

domain of definition not fulfilled, gnathiferans

misscored

domain of definition not fulfilled

domain of definition not fulfilled

domain of definition not fulfilled in the analyses of Ax and Eernisse et al. (1992), and several misscorings in Eernisse et al. (1992) and Zrzavý et al. (1998)

may be homoplastic or symplesiomorphic (eutelic taxa)

domain of definition not fulfilled in the analyses of Ax and Eernisse et al. (1992), and several phyla misscored in Eernisse et al. (1992) and Zrzavý et al. (1998)

Ok when plathelminthomorphs are nested within Bilateria

misscored for plathelminthomorphans and gastrotrichs

symplesiomorphy or homoplasy; platyhelminth ground pattern uncertain

Ok when plathelminthomorphs are nested within Bilateria

homoplastic

domain of definition not fulfilled; various misscorings and problematic character coding

may be characteristic of more taxa

scored "?" for gnathostomulids and several platyhelminth taxa

ground patterns of gnathostomulids and

platyhelminths not similar

gnathostomulids lack biflagellate sperm

homoplastic, and gnathostomulids may possess a

compact acrosome

not independent from $\mathrm{P} 20$

many misscorings and scored "?" for gnathostomulids and several platyhelminths

symplesiomorphy or homoplasy and incorrect character coding and scoring for several phyla Z258 conflicts with Z132, several taxa are misscored various misscorings

scoring Cycliophora problematic

based on the unsupported homology of presumed planktotrophic polyclad and pilidium larvae similar situation encountered in basal molluscs such as aplacophorans and polyplacophorans

Ok, but not unique 
Table 2. Continued.

\begin{tabular}{|c|c|c|}
\hline Sister taxon & Synapomorphies and source & Comments \\
\hline .. & $\begin{array}{l}\text { general shape of larval ciliary bands (Nielsen, } \\
\text { 1995, 2001) } \\
\text { larvae or adult with downstream-collecting ciliary } \\
\text { bands of compound cilia on multiciliate cells (N22) } \\
\text { larva with strongly reduced hyposphere (S28; NI20) }\end{array}$ & $\begin{array}{l}\text { based on the unsupported homology of presumed } \\
\text { planktotrophic polyclad and pilidium larvae } \\
\text { based on the unsupported homology of presumed } \\
\text { planktotrophic polyclad and pilidium larvae and } \\
\text { highly homoplastic } \\
\text { based on the unsupported homology of presumed } \\
\text { planktotrophic polyclad and pilidium larvae }\end{array}$ \\
\hline Various protostomes & $\begin{array}{l}\text { spiral quartet cleavage (ZI1) } \\
\text { lophotrochozoan complement of Hox genes (ZI59) } \\
4 \mathrm{~d} \text {-mesoderm (ZII7) } \\
\text { loss of non-ciliated gut (ZII49) } \\
\text { loss of non-ciliated pharynx (ZII51) } \\
\text { protonephridial terminal cells monociliated } \rightarrow \text { bi- } \\
\text { and multiciliated (ZII68) } \\
\text { frontal gland system (ZII 105) } \\
\text { cleavage pattern fundamentally spiral (BI18) } \\
\text { synaptic nervous system concentrated ventrally } \\
\text { or ventrolaterally (BI52) } \\
\text { entomesoderm derives from a single (mesentoblast) } \\
\text { cell, typically the } 4 \mathrm{~d} \text { cell (BI74) } \\
\text { sheets of subepidermal muscle derived at least in } \\
\text { part from } 4 \mathrm{~d} \text { mesoderm (BI75) }\end{array}$ & $\begin{array}{l}\text { ok, convergent with Gnathostomulida } \\
\text { equivocal } \\
\text { ok } \\
\text { ok, but nemertodermatids misscored } \\
\text { ok, but equivocal } \\
\text { ok, but different semaphoront choice may result in } \\
\text { different scoring for some taxa } \\
\text { ok, but Cycliophora misscored } \\
\text { misscored for syndermates and panarthropods } \\
\text { ok, but coding may be changed } \\
\text { misscored for panarthropods } \\
\text { misscored for panarthropods }\end{array}$ \\
\hline $\begin{array}{l}\text { Coelomate } \\
\text { protostomes }\end{array}$ & $\begin{array}{l}\text { cerebral ganglion and orthogonal nervous system } \\
\text { (B64c) } \\
\text { 4d- derived subepidermal muscle sheets (B58) } \\
\text { mesentoblast derived mesoderm (B18) } \\
\text { typical spiral cleavage (B13b) }\end{array}$ & $\begin{array}{l}\text { domain of definition not fulfilled, and uncertainty } \\
\text { over platyhelminth ground pattern } \\
\text { domain of definition not fulfilled } \\
\text { domain of definition not fulfilled } \\
\text { domain of definition not fulfilled }\end{array}$ \\
\hline
\end{tabular}

(adult ability to move) for all taxa that are typically sessile as adults. Three of the four synapomorphies that placed the platyhelminths as a sister group to the remaining protostomes in Brusca \& Brusca (2003) are misscored for both the syndermates and panarthropods (BI18), or the panarthropods only $(B I 74,75)$. While the analyses of Meglitsch \& Schram (1991), Eernisse et al. (1992), and Zrzavý et al. (1998) suggested that absence of an anus may be an autapomorphy of plathelminthomorphs, Nielsen (2001) misscored NI32 (mouth and anus) for the platyhelminths, while interpreting the gnathostomulids to have an anus. However, experimental rescoring of either the platyhelminths alone, or together with the gnathostomulids for lack of a true anus does not change the topology of the strict consensus of Nielsen (2001). Finally, the validity of the synapomorphies proposed in support of a clade Parenchymia by Nielsen (1995), Nielsen et al. (1996), and Sørensen et al. (2000), which was based upon larval similarities of presumed planktotrophic polyclad and pilidium larvae, is predicated upon the assumption that these larval types are parts of the ground patterns of the respective phyla. As argued under Larva with strongly reduced hyposphere, this premise is unsupported.

Finally, it may be illuminating to compare the diagnostic apomorphies of the proposed clades in terms of their biological significance. This may yield additional insights into the quality of the apomorphies, either as truly independent phylogenetic markers, or perhaps as (partly) correlated elements of one or a few adaptive complexes (see Arnold, 1990 and Lee, 1998b for examples of such character quality assessment). A significant proportion of the characters proposed to unite the platyhelminths with 
either the gnathiferans or the gnathostomulids refer to the reproductive system, notably internal sperm deposition, internal fertilization, and filiform sperm. Any confidence we might have in the value of these features as independent synapomorphies should be tempered by the probability that they are functionally correlated with the possession of small body size (see Olive, 1985 for a review of covariability of reproductive traits). A change to small body size may result in the loss of the ability to produce large quantities of gametes that can be freely discharged into the environment. Small bodied taxa may shift towards specialized modes of sperm transfer, internal fertilization, and the consequent evolution of modified sperm morphology to ensure fertilization success. For example, Ponder \& Lindberg (1997) convincingly showed that the gastropods frequently evolved direct sperm transfer through copulation, internal fertilization, and modified filiform sperm morphology, and that these correlated changes constitute one of the major trends of gastropod evolution. The perceived independence of these correlated characters may thus be misleading.

In conclusion, current morphological analyses have yielded very little unambiguous support for an inarguable placement of platyhelminths within the Metazoa. None of the six synapomorphies suggested in support of a clade of the platyhelminths and gnathiferans is compelling. The Plathelminthomorpha hypothesis appears to be the most prominently supported hypothesis, as judged by the number of independent studies that support this grouping and the proposed number of diagnostic apomorphies (Table 2). However, the only potential unique synapomorphy of the platyhelminths and the gnathostomulids is the loss of an anus if plathelminthomorphans are nested deeply within the Bilateria (characterized by possession of a unidirectional gut with an anus). However, the interpretation of the presence or absence of an anus in the gnathostomulids is debatable, and as is shown under Gnathostomulida, clade support for Plathelminthomorpha is not always robust in the face of experimental manipulation of the data matrix.

The only unique characters marshaled in support of the Parenchymia hypothesis by Nielsen
(1995), Nielsen et al. (1996), and Sørensen et al. (2000) are contradicted by the likely derived nature of presumed planktotrophic polyclad and pilidium larvae.

This leaves a clade of protostome phyla of unknown membership as currently the most likely sister group to the platyhelminths. The prevalence of scoring errors and differences in character selection between studies have so far prevented an effective cladistic test of alternative phylogenetic placements of the platyhelminths. In order to identify the platyhelminth sister clade a new analysis has to be performed that includes all pertinent and properly scored character information. The possession of spiral cleavage and $4 \mathrm{~d}$-mesoderm indicate that the platyhelminths (excluding the acoelomorphs) are part of a larger spiralian clade. Although spiral quartet cleavage can reasonably be assumed to be in the ground pattern of the platyhelminths (having been indeed observed in polyclads, lecithoepitheliates, and proseriates with hints in the macrostomids; Galleni \& Gremigni, 1989), detailed studies of early development and cell lineages (including mesoderm source) are so far limited to the polyclads (Thomas, 1986; Boyer et al., 1996a; Boyer et al., 1998). This leaves the platyhelminth ground pattern as uncertain.

\section{Placing Platyhelminthes in molecular and total evidence analyses}

Phylogenetic analyses of $18 \mathrm{~S}$ rDNA sequences, or myosin heavy chain type II sequences (Ruiz-Trillo et al., 2002) have not led to the identification of a reliable platyhelminth sister group, but we do see some consensus about the overall position of the platyhelminths in the Metazoa. The earliest analyses supported a position of the platyhelminths at the base of the Bilateria (e.g. Field et al., 1988), lending apparent support to the widespread textbook view of animal phylogeny (see Jenner, 2000 for a critique of this view). While this position was more or less maintained in further analyses with increased taxon sampling (Eernisse, 1997; Lipscomb et al., 1998; Winnepenninckx et al., 1998; Peterson \& Eernisse, 2001), the latest most comprehensive analyses instead support a lophotrochozoan affin- 
ity for the platyhelminths (Littlewood et al., 1998; Ruiz-Trillo et al., 1999, 2002; Giribet et al., 2000; Baguñà et al., 2001; Littlewbod \& Olson, 2001; Zrzavý et al., 2001; Jondelius et al., 2002). However, recent phylogenetic analysis of amino acid sequences of various nuclear genes also supports a basal bilaterian position of platyhelminths (Hausdorf, 2000), but these results should be interpreted with extreme caution because the sampling of metazoans is equally as restricted as the earliest $18 \mathrm{~S}$ studies that yielded spurious results. Only the mouse, human, Drosophila melanogaster, Caenorhabditis elegans, and the trematode Schistosoma are included. The study by Aguinaldo et al. (1997) emphasized that only platyhelminths with slowly evolving $18 \mathrm{~S}$ rDNA sequences should be included in phylogenetic analyses to prevent spurious results caused by long branch attraction. The lophotrochozoan placement of the platyhelminths is consistent with data on the complement of Hox genes present in rhabditophoran platyhelminths and mitochondrial gene arrangements (Balavoine, 1997, 1998, Rosa et al., 1999; Nickisch-Rosenegk et al., 2001). However, this conclusion only holds for Catenulida + Rhabditophora. It should be noted that the most comprehensive phylogenetic analysis of $18 \mathrm{~S}$ sequences to date places the platyhelminths with the acanthocephalans, mesozoans, gastrotrichs and nematomorphs at the base of clade of non-ecdysozoan bilaterians (Peer et al., 2000).

The acoels and nemertodermatids may be the earliest branching crown group bilaterians (RuizTrillo et al., 1999, 2002; Jondelius et al., 2002; Telford et al., in press). This hypothesis is supported by a variety of molecular data sources, including $18 \mathrm{~S}$ rDNA, 28S rDNA, and myosin II sequences. Phylogenetic analysis of amino acid sequences of elongation factor 1-alpha was reported to contradict the basal position of Acoela among the Bilateria by Berney et al. (2000). However, reanalysis of this and new data has led Littlewood et al. (2001) to the conclusion that, when considered in isolation, elongation factor 1-alpha sequences contain insufficient signal for a reliable placement of the acoels. Giribet et al. (2000) and Peterson \& Eernisse (2001) are skeptical about the basal position of the acoels found by Ruiz-Trillo et al. (1999), pointing to the possibility of long branch attraction of the acoel sequences to the long branch separating nonbilaterians and bilaterians. This criticism is probably not entirely justified. Ruiz-Trillo et al. (1999) performed a relative rate test to ascertain that only taxa with similar substitution rates were included in the analysis. Consequently, only the acoel Paratomella rubra was included in the final analysis. In contrast, Giribet et al. (2000) used two species that were not tested for their substitution rates, and Peterson \& Eernisse (2001) included three acoels that were rejected in the relative rate test by RuizTrillo et al. (1999). It can thus be concluded that long branch attraction of acoels to the base of the Bilateria is a possibility in the analyses of Giribet et al. (2000) and Peterson \& Eernisse (2001), but it is unwarranted to extend this criticism to the study of Ruiz-Trillo et al. (1999).

It is interesting to note that metazoan cladists solely interpret long branches as an indication of possible trouble. However, it could be argued instead that long branches may be expected to occur in particular in related taxa. The known distribution of short and long branch taxa for diverse organisms with entirely reasonable clusterings of the latter support this conjecture (Siddall, 1998). However, given current methods it is virtually impossible to decide beyond reasonable doubt whether a grouping of long branch taxa is artifactual or not.

The position of nemertodermatids is unreliable in the analyses of Zrzavý et al. (1998), Littlewood et al. (1999a, b), and Giribet et al. (2000) because the included 18S rDNA sequence of Nemertinoides elongatus turned out to be a sequence artifact (see also Jondẹlius et al., 2002). New nemertodermatid $18 \mathrm{~S}$ rDNA and mitochondrial sequence data are reported to support their position as basal crown group bilaterians branching off immediately after the acoels as the sister group to the remaining Bilateria (Baguñà et al., 2001; Jondelius et al., 2002; Telford et al., in press). New myosin II data also supports both acoels and nemertodermatids as basal crown bilaterians, while indicating that Acoelomorpha may be monopyletic, and these data are furthermore free from potential long branch attraction problems because the analyzed taxa show comparable substitution rates (Ruiz-Trillo et al., 2002).

So far, no clear picture about the sister group of platyhelminths has emerged from total evidence 
analyses of $18 \mathrm{~S}$ rDNA sequence data and morphology (Fig. 6). Zrzavý et al. (1998) place a paraphyletic platyhelminth grade at the base of the Bilateria. Giribet et al. (2000) and Zrzavý et al. (2001) place the platyhelminths (acoelomorphs excluded in the latter analysis) within a platyzoan clade either as part of the Protostomia (Giribet et al., 2000) or in an unresolved position (Zrzavý et al., 2001). Peterson $\&$ Eernisse (2001) determined a lophotrochozoan affinity of Catenulida + Rhabditophora, with acoelomorphs at the base of the Bilateria. The total evidence analysis of Zrzavý (2003) placed the acoels and nemertodermatids at the base of the Bilateria. Both of these taxa were represented by only a single sequence, and the nemertodermatid sequence Meara stichopi) was labeled as problematic by Zrzavý. Neither did another Meara sequence pass the relative rate test in Jondelius et al. (2002). Therefore Zrzavý's (2003) results should be considered with caution. Zrzavý (2003) placed Catenulida + Rhabditophora as a sister clade to a clade including the nemerteans, neotrochozoans, entoprocts, and $\mathrm{Lo}$ batocerebrum.

In conclusion, molecular sequence data indicates the probability of a basal bilaterian position of the acoelomorphs, with the remaining platyhelminths as probably lophotrochozoan protostomes. Total evidence analyses are in broad agreement with these results, but an exact sister group for Catenulida + Rhabditophora has not been revealed. A striking conclusion is that molecular sequence data currently do not clearly support any of the sister group relationships proposed on the basis of morphological evidence. The $18 \mathrm{~S}$ analyses of Giribet et al. (2000) and Zrzavý et al. (2001) come closest to the morphological hypothesis proposed by Ahlrichs (1995), who on the basis of a manual cladistic analysis concluded that the platyhelminths group with the gnathiferans, although the molecular data additionally suggest a potential close relationship with the gastrotrichs, myzostomids, and cycliophorans.

\section{Alternative Phylogenetic Hypotheses for Nemertea}

Two conflicting phylogenetic placements of nemerteans, the ribbon or proboscis worms, that have recently been defended on the basis of morpho- logical data merit special attention. The first postulates a close relationship to the Platyhelminthes, the second to a larger clade of coelomate spiralians that may or may not include panarthropods. Support for these contending hypotheses can largely be reduced to favoring the importance of either larval similarities between nemertean pilidium larvae and polyclad larvae, or the ontogenetic and structural similarity of nemertean rhynchocoel and blood vessels, and the coeloms found in coelomate spiralians. As is argued below, I think that the latter hypothesis is better supported, but difficulties with the interpretation of characters so far only allow a tentative conclusion.

\section{(Nemertea Platyhelminthes)}

See under Platyhelminthes.

\section{(Nemertea Annelida)}

Proposed synapomorphies: Cavalier-Smith (1998). Cavalier-Smith (1998) united these two phyla under the new name Vermizoa.

Cavalier-Smith (1998)

- closed blood vessels

- ciliated larvae without bivalved shells

- two ventrolateral or one primitively paired ventral nerve cord

\section{Comments}

Cavalier-Smith (1998) proposed a new taxon Vermizoa comprising Nemertea and Annelida, chiefly on the basis of the shared possession of "well-developed closed vascular systems." Although Cavalier-Smith (1998: 242) stated that “...there are no strong morphological reasons against grouping annelids and nemerteans in the same superphylum," we have to conclude that convincing evidence in favor of this grouping is also lacking. Apart from the fact that closed blood vascular systems also occur in a number of bilaterian phyla that can be considered as closely related to the annelids, current information on the development, ultrastructure, and 


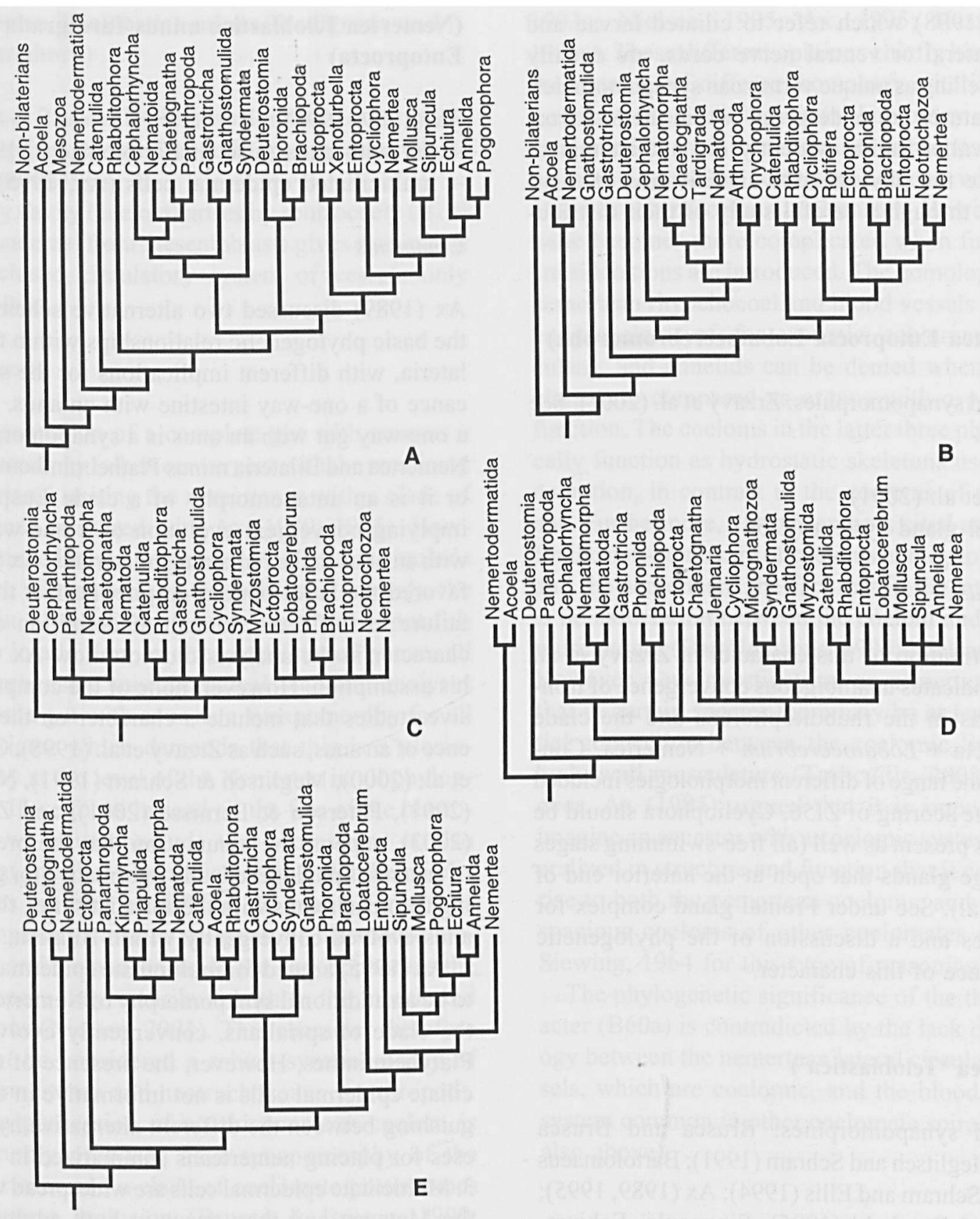

Fig. 6. Phylogenetic position of the platyhelminths, gnathostomulids, and nemerteans according to total evidence analyses. (A) Zrzavý et al. (1998); (B) Peterson \& Eernisse (2001); (C) Zrzavý et al. (2001); (D) Zrzavý (2003); (E) Giribet et al. (2000).

anatomical location of nemertean circulatory vessels strongly support their interpretation as coeloms. This forecloses homology with the blood vascular system of annelids or other bilaterians (Ruppert \& Carle, 1983; Turbeville \& Ruppert, 1985; Turbeville,
1986). This information also necessitates a reassessment of characters B60a (Brusca \& Brusca, 1990) and H23 (Haszprunar, 1996a) that are based on the same unfounded homology assumption.

The other two characters proposed by Cavalier- 
Smith (1998) which refer to ciliated larvae and ventrolateral or ventral nerve cords, are equally uncompelling as unique vermizoan synapomorphies. Both features are widespread within the Metazoa, and Cavalier-Smith evidently did not attempt to elucidate the phylogenetic significance of these features through a careful study of their distribution.

\section{(Nemertea Entoprocta Lobatocerebromorpha)}

Proposed synapomorphies: Zrzavý et al. (2001). See figure 4.

Zrzavý et al. (2001)

- frontal gland system (ZI56)

\section{Comments}

The distribution of this character in Zrzavý et al. (2001) indicates unambiguous convergence of frontal glands in the rhabditophorans and the clade Entoprocta + Lobatocerebrum + Nemertea. Considering the range of different morphologies included within the scoring of ZI56, Cycliophora should be scored as present as well (all free-swimming stages have large glands that open at the anterior end of the animal). See under Frontal gland complex for references and a discussion of the phylogenetic significance of this character.

\section{(Nemertea 'Teloblastica')}

Proposed synapomorphies: Brusca and Brusca (1990); Meglitsch and Schram (1991); Bartolomaeus (1993a); Schram and Ellis (1994); Ax (1989, 1995); Rouse and Fauchald (1995). Sipuncula, Echiura, Annelida, Mollusca, and Arthropoda form the core taxa of a sister clade to Nemertea in these studies. The clade may additionally include Entoprocta, Onychophora, and Tardigrada depending on which analysis is consulted. See figures 2 and 4.

\section{(Nemertea Teloblastica minus Tardigrada plus Entoprocta)}

Ax $(1989,1995)$

- gut with anus (also in Bartolomaeus, 1993a)

- multiciliated epidermal cells (Ax, 1995)

\section{Comments}

Ax (1989) discussed two alternative schemes for the basic phylogenetic relationships within the $\mathrm{Bi}$ lateria, with different implications for the significance of a one-way intestine with an anus. Either a one-way gut with an anus is a synapomorphy of Nemertea and Bilateria minus Plathelminthomorpha, or it is an autapomorphy of a clade Euspiralia, implying convergent evolution of a one-way gut with an anus in the remaining bilaterians. Ax (1995) favored the latter option, despite the fact that the failure to fulfill the domain of definition of this character in the analysis did not allow Ax to test his assumption. However, none of the comprehensive studies that include a character on the presence of an anus, such as Zrzavý et al. (1998), Giribet et al. (2000), Meglitsch \& Schram (1991), Nielsen (2001), Peterson \& Eernisse (2001), and Zrzavý (2003), support the assumption that the presence of an anus is phylogenetically informative for grouping the nemerteans with the spiralians, i.e., that the anus evolved convergently within Bilateria.

Ax (1995) argued for multiciliate epidermal cells to be an additional synapomorphy of Nemertea and the clade of spiralians, convergently evolved in Platyhelminthes. However, the presence of multiciliate epidermal cells is not informative in distinguishing between the different alternative hypotheses for placing nemerteans summarized in Table 3. Multiciliate epidermal cells are widespread within the Metazoa and they occur in both adults, and especially larvae, where they often make up the larval locomotory and feeding ciliary bands (Nielsen, $1987,1995)$, in particular in the protostome phyla that are commonly hypothesized to be closely related to the nemerteans. 


\section{(Nemertea Teloblastica minus Tardigrada and Onychophora)}

Brusca \& Brusca (1990) (also Sundberg et al., 1998)

- complete gut with mouth arising from blastopore (B10b)

- body cavity (coelom) arises by schizocoely (B19)

- mesoderm (from mesentoblast) gives rise in part to closed circulatory system of vessels only (B60a)

\section{Comments}

The postulation of a complete gut with anus as a synapomorphy (albeit convergent with the remaining bilaterians) uniting the nemerteans with a clade of spiralians is shared with Ax $(1989,1995)$. However, since Brusca \& Brusca (1990) did not include all taxa with an anus in their cladistic analysis, the domain of definition for this feature was not fulfilled. In addition, as already discussed above, all comprehensive morphological cladistic analyses support a gut with anus as a synapomorphy for all bilaterians, which demands that this is a plesiomorphy at the level of the Nemertea in all phylogenies. If we focus instead on the fate of the blastopore, we must conclude that protostomy is also present in other phyla such as the platyhelminths (just as in many nemerteans the mouth forms as a new opening close to the point of blastopore closure), and the phoronids. In the onychophorans the blastopore may contribute both to the mouth and the anus (Nielsen, 2001). This character therefore cannot be considered a robust synapomorphy of the nemerteans and neotrochozoans + arthropods.

The possession of a schizocoelous coelom is commonly proposed as a synapomorphy of the nemerteans and a clade of coelomate protostomes of diverse inclusion (Brusca \& Brusca, 1990; Eernisse et al., 1992; Rouse \& Fauchald, 1995). This hypothesis is supported by the interpretation of the nemertean rhynchocoel and lateral vessels as coeloms. However, there is no universal agreement on this point. Some authors, while conceding the general coelomic histological organization of nemertean blood vessels and rhynchocoel nevertheless argue a priori for the autapomorphic nature of the nemertean coeloms (Bartolomaeus, 1993a; Nielsen, 1995; Ax, 1995; Haszprunar, 1996a). These different opinions chiefly hinge upon the adoption of different homology criteria. When a structural or developmental homology criterion is adopted, there is no reason not to propose potential homology of the nemertean coeloms with those of other schizocoelous coelomates. However, the issue becomes more complicated when functional considerations are introduced. The homology of the nemertean rhynchocoel and blood vessels with the body coeloms of, for example, echiurans, sipunculans, and annelids can be denied when the coeloms are compared as organs with a particular function. The coeloms in the latter three phyla typically function as hydrostatic skeletons used in locomotion, in contrast to the coeloms of nemerteans. Furthermore, various authors cited as support for the non-homology of coeloms in the protostomes and the nemerteans the fact that in the latter there is no association between the coelom and somatic musculature (Bartolomaeus, 1994; Nielsen, 1995). However, new investigations on nemerteans show that in certain species there may be at least a partial connection between the coelomic lining and body wall musculature (Turbeville, 2002). Moreover, Ax (1995) argued that it is impossible to imagine an ancestor with a coelomic system so generalized in structure and function that it could give rise to both the nemertean coeloms, and the more spacious coeloms of other coelomates (see also Siewing, 1964 for this type of reasoning).

The phylogenetic significance of the third character (B60a) is contradicted by the lack of homology between the nemertean lateral circulatory vessels, which are coelomic, and the blood vascular system common in other coelomate spiralians (see also above).

\section{(Nemertea Teloblastica)}

Meglitsch \& Schram (1991)

- segmented or serial structures derived from mesoderm (M43)

$\bullet$

Nemerteans are primitively unsegmented animals. The only ground pattern mesodermal structures that are serially repeated are the gonads (Goodrich, 1895; 
gonads were interpreted as coeloms by Turbeville \& Ruppert, 1985; see also discussion in Bartolomaeus, 1999). The serially repeated transverse connectives between the lateral coelomic "blood vessels" in some nemertean species are without counterpart in other phyla and therefore phylogenetically uninformative. Consequently, the only shared similarity of serially repeated mesodermal structures in the nemerteans and annelids are the gonads. However, it is far from obvious how such a comparison could be extrapolated to other protostomes. For a meaningful phylogenetic character, it is essential to specify what mesodermal derivatives (muscles, gonads) are compared (see also Budd, 2001 for a discussion of seriality and segmentation in protostomes). Just as it is not likely that all mesodermal structures within a single organism are homologous, so too is it not straightforward to suggest homology of serially repeated mesodermal structures in different species regardless of which organs are compared. Because gonads are the only serially repeated mesodermal organs in the nemertean ground pattern we have to limit our comparison to gonads in the sister clade of the nemerteans proposed in Meglitsch \& Schram (1991). The first three taxa that branch off within the sister clade of the Nemertea in Meglitsch \& Schram (1991) are Mollusca, Echiura, and Sipuncula. Within the molluscs serially repeated gonads (two to four pairs) can be found only in the monoplacophorans. However, their derived phylogenetic position within the molluscs indicates that monoplacophorans are not particularly informative for the molluscan ground pattern. Serially repeated gonads can be excluded from the ground patterns of the echiurans and sipunculans. Consequently, M43 is not a compelling synapomorphy of nemerteans and Teloblastica.

\section{(Nemertea Teloblastica minus Tardigrada)}

Rouse \& Fauchald (1995)

- schizocoel (RI2)

- anus (RI5)

The interpretation of a schizocoel as a synapomorphy of Nemertea and a clade of coelomate protostomes is justified on the basis of current information, and is in agreement with the results of other studies (Brusca \& Brusca, 1990; Eernisse et al., 1992).

Although the domain of definition for the presence of an anus is not fulfilled in this study, similar to the situation observed in Ax $(1989,1995)$ and Brusca \& Brusca (1990), the interpretation of an anus as a synapomorphy of the nemerteans and a clade of teloblasticans would imply convergent evolution of the anus in aschelminths and deuterostomes. This is contradicted by all cladistic analyses with a comprehensive phylum sampling.

\section{(Nemertea 'Neotrochozoa')}

Proposed synapomorphies: Eernisse et al. (1992); Haszprunar (1996a); Zrzavý et al. (1998); Giribet et al. (2000); Peterson \& Eernisse (2001); Zrzavý (2003). Sipuncula, Mollusca, Echiura, Annelida form the core taxa of a sister clade to Nemertea in these studies. This clade may also include as separate taxa Pogonophora, Myzostomida, and Entoprocta. See figures 2,4 , and 5 .

\section{Neotrochozoa (plus Pogonophora)}

Eernisse et al. (1992)

- entomesoblast proliferation contributing to mesoderm (E7)

- schizocoelous formation of body cavity lined with mesodermal peritoneum (E17)

- swimming/feeding band(s) of cilia in larvae with compound cilia (E39)

- pelagic larvae with apical ciliary tuft and plate (E41)

- cerebral rhabdomeric larval ocelli or integumentary pigment cups (E43)

- dermal circular (or external transverse) muscular fibers (E131)

\section{Comments}

The presence of mesoderm derived from an entomesoblast (4d-mesentoblast) is certainly shared between the nemerteans and neotrochozoans (Biggelaar et al., 1996; Biggelaar et al., 1997), but problems of character coding and scoring mask the plesiomorphic nature of E7. It is misscored for the 
rhabditophoran platyhelminths, which do possess a 4d-mesentoblast (data mostly based on polyclads: Thomas, 1986; Boyer, 1997: Henry \& Martindale, 1997; Boyer et al., 1998). Recent findings on cell lineage in the acoel Neochildia fusca did allow the determination of endomesoderm, but did not indicate the presence of a $4 \mathrm{~d}$-mesentoblast (Henry et al., 2000). Phylogenetic information for resolving relationships between spiralians may reside in the mode and timing of mesentoblast formation (Biggelaar et al., 1996, 1997; Guralnick \& Lindberg, 2001), and it has been suggested on the basis of such data that the nemerteans are more closely related to the molluses and annelids than any of these phyla is to platyhelminths (Loon \& Biggelaar, 1998). However, a rigorous cladistic evaluation of mesentoblast timing in different phyla is still wanting (Nielsen \& Meier, 2002; but see Guralnick \& Lindberg, 2002). Interestingly, the scoring of E5 (entomesoblast cell 4d), and E7 (entomesoblast proliferation contributing to mesoderm) should be identical for taxa with an entomesoblast. Instead, the scoring is in conflict for the rhabditophoran platyhelminths, crustaceans, uniramians, chelicerates, and tardigrades. For these taxa the entomesoblast is scored as being present (incorrectly so for the arthropods and tardigrades; the ground patterns of these taxa lack spiral cleavage and a 4d-mesentoblast: Anderson, 1973; Scholtz, 1997; see discussion under Secondary body cavity, coelom, ontogenetic source), but mesoderm derived from that cell as being absent. Additionally, Entoprocta was not included in Eernisse et al.'s study despite the presence of mesentoblast derived mesoderm in this phylum (Nielsen, 1995, 2001).

The presence of a schizocoel lined by a mesodermal peritoneum may indeed unite the nemerteans and coelomate protostomes, exclusive of platyhelminths (see also Brusca \& Brusca, 1990; Rouse $\&$ Fauchald, 1995). However, the scoring for several taxa such as the arthropods and onychophorans should be reconsidered (scored as lacking a schizocoel or '?' respectively), and the inclusion of a peritoneum (non-contractile epithelium overlying myoepithelial or muscle cells) in the character definition is unnecessarily complicated and potentially misleading, since it may have evolved convergently within various monophyletic phyla, e.g., the Annelida: Bartolomaeus (1994). The reader is referred to the section below on Secondary body cavity, coelom, for a justification of these conclusions, and a complete discussion of the scoring of coelom characters across the Metazoa.

The value of the larval characters E39, E41, and E43 for placing the nemerteans among the spiralians is dependent on the postulation of pilidium larvae in the ground pattern of the phylum. Current information suggests this is unlikely. In particular, a recent molecular phylogeny of the nemerteans based upon four different genes (the nuclear genes for 28S rRNA and histone $\mathrm{H} 3$, and the mitochondrial genes for $16 \mathrm{~S}$ rRNA and cytochrome $c$ oxidase subunit I) strongly supports the evolution of the pilidium larva within the phylum Nemertea (Thollesson \& Norenburg, 2003). The palaeonemerteans, which lack pilidium larvae, form a basal paraphyletic stem lineage to the rest of the nemerteans. The only palaeonemertean with a pilidium larva in the life cycle (Hubrechtella dubia) now forms the sister group to the monophyletic heteronemerteans. Thollesson \& Norenburg (2003) name this new clade Pilidiophora based upon the unique shared possession of pilidium larvae.

It should be noted that an apical organ (E41) also appears to be routinely present in the 'larvae' (juveniles) of direct developing nemerteans (Hyman, 1951a; Iwata, 1968; Cantell, 1989; Turbeville, 1996; Henry \& Martindale, 1997), and may therefore be plesiomorphic for the nemerteans. I could only find support for the presence of 'larval' ocelli (E43) in directly developing nemerteans and in post-metamorphic juveniles of indirectly developing nemerteans, which leads to the conclusion that ocelli are not a larval nemertean trait.

The phylogenetic significance of circular dermal (body wall) muscles (E131) needs to be reassessed for two reasons. First, various taxa were dubiously or incorrectly scored, including the acoelomorphs, rhabditophorans, and gnathostomulids. Despite the scoring of E131, these taxa do possess circular body wall muscles, although they may be rather weakly developed and present only locally as in gnathostomulids (Hyman, 1951a; Lammert, 1991; Rieger et al., 1991b; Ladurner \& Rieger, 2000; Rieger \& Ladurner, 2001; Tyler, 2001; 
Tyler \& Hooge, 2001). Polyplacophorans and conchiferans were also scored as lacking circular components in the body wall musculature, in contrast to the aplacophorans which may represent the plesiomorphic condition for Mollusca (Haszprunar \& Wanninger, 2000). In fact, within the molluscs only the aplacophorans (Scheltema et al., 1994) possess all components of a typical orthogonal muscle grid that is characteristic of both coelomate and non-coelomate vermiform metazoans (including outer circular and inner longitudinal muscles; see Rieger \& Ladurner, 2001). However, it should be noted that recent studies on the embryology of molluscan muscle systems have shown that circular or ring-like muscle components are discernable in the body wall of developing gastropods, scaphopods, and polyplacophorans (Wanninger et al., 1999; Haszprunar \& Wanninger, 2000; Wanninger \& Haszprunar, 2002; P. Damen, pers. comm.). Nevertheless, the evolution of these muscles is currently far from clear, and a resolution of this problem is complicated by the fact that larval and adult molluscan muscle systems may be remarkably independent. Data on the early ontogeny of muscle systems in different phyla may yield as yet unexplored but promising sources of phylogenetic information that may help resolve the relationships of spiralians. For example, the relative timing of the development of circular and longitudinal muscle fibers may differ between phyla, with the acoel Convoluta pulchra first forming circular fibers followed by the development of longitudinal fibers, while the polychaete Capitella sp. first forms longitudinal fibers (Ladurner \& Rieger, 2000; Hill \& Boyer, 2001). The degree of association between larval and adult muscle systems might also provide valuable phylogenetic data. For example, the high degree of independence between larval and adult muscle systems in the molluscs (Wanninger et al, 1999; Haszprunar \& Wanninger, 2000) appears to be contrasted by the close association of these systems in the recently investigated acoels and polychaetes, where the larval muscle system appears to forms a grid for the development of the adult muscles (Ladurner \& Rieger, 2000; Hill \& Boyer, 2001).

Second, various taxa that also possess more or less clearly defined circular body wall muscles, which are sometimes restricted to particular body regions such as the pharynx or proboscis, were not included in the analysis of Eernisse et al. (1992). The result is that the domain of definition for this character was not fulfilled (e.g. gastrotrichs, hemichordates, chaetognaths, rotifers: Hyman, 1959; Clément \& Wurdak, 1991; Ruppert, 1991b; Lorenzen, 1996c; Shinn, 1997; Hochberg \& Litvaitis, 2001b).

\section{Neotrochozoa (plus Myzostomida)}

\author{
Haszprunar (1996a) \\ - compound cilia (H5) \\ - blood pigments (H24) \\ - only testis sacular $\rightarrow$ both testis and ovarium \\ sacular (H31)
}

\section{Comments}

Since compound cilia are only reported for pilidium larvae (Nielsen, 1987), which are not plesiomorphic for the nemerteans, this is a weak character for resolving the phylogenetic position of Nemertea. Because most alternative phylogenies (see Table 3) place the nemerteans at the base of a clade that is likely characterized by compound cilia, this trait will not be very helpful in arbitrating between available alternative phylogenetic hypotheses.

Haszprunar's character on respiratory pigments should be recoded and rescored for several taxa. First, it unites non-homologous oxygen-transporting proteins (see discussion under Respiratory pigments). Second, the entoprocts appear to be misscored because so far no respiratory proteins are known in that phylum (Terwilliger, 1998; C. Nielsen, pers. comm.). Third, the domain of definition is not fulfilled. Even when $\mathrm{H} 24$ is recoded to create separate characters for arguably homologous molecules, such as hemoglobin and hemerythrin, a number of taxa that possess either of these proteins is not included in the data matrix, e.g., Priapulida, Brachiopoda, Echinodermata, and Chordata (Terwilliger, 1998). We must conclude that the phylogenetic significance of this character needs to be reassessed. 
The transformation of $\mathrm{H} 31$, from the presence of sacular testes only, to the presence of both sacular testes and ovaries is ambiguouts. More importantly, the rhabditophorans were incorrectly scored as possessing asacular gonads, and the domain of definition of this character is clearly not fulfilled for H31. A proper evaluation of Haszprunar's hypothesis that sacular gonads (both testes and ovaries) characterize "in a very broad sense" (Haszprunar, 1996a: 17) a coelomate condition necessitates the inclusion of many bilaterian phyla not included in his analysis (see under Gonads asacular or sacular for further discussion of this character in other cladistic analyses).

\section{Neotrochozoa (plus Myzostomida and Pogonophora)}

\author{
Zrzavý et al. (1998) \\ - coelom as defined histologically (Z30) \\ - gonocoel (Z31) \\ - coelomocytes (Z33) \\ - podocytes (Z43) \\ - respiratory pigments (Z44) \\ - adult brain derived from/associated with larval \\ apical organ/apical pole (Z145) \\ - serial repetition in nerve collaterals (Z236)
}

\section{Comments}

The presence of a histologically defined coelom may be a proper (albeit convergent) synapomorphy of the nemerteans and other coelomate protostomes. It should be noted that in contrast to Z30 there is no support for the scoring of a histologically defined coelom in the ground patterns of Urochordata, Priapulida, and Tardigrada (see under Secondary body cavity, coelom for justification of this conclusion).

As is discussed under Gonocoel, the scoring and phylogenetic significance of such a cavity should be thoroughly reevaluated. The current scoring of $\mathrm{Z} 31$ is a poor reflection of the comparative morphology of metazoan gonads.

The scoring of Z33 does not reflect the widespread distribution of coelomocytes in almost all of the coelomate Metazoa. As is discussed below under Coelomocytes, the phylogenetic significance of these cells remains to be determined.

Podocytes could be a synapomorphy of the nemerteans and other coelomate protostomes, but convergence is likely, and even plesiomorphy of this character on this phylogenetic level cannot be ruled out. However, in order to reconstruct the proper pattern of podocyte distribution in the Metazoa, Z43 needs to be rescored for a number of phyla that are misscored as lacking podocytes (see under Podocytes/terminal cells/nephrocytes for discussion).

The phylogenetic significance of respiratory pigments has not been properly assessed in Zrzavý et al. (1998), nor in any of the other recent cladistic analyses that included a character on their presence, and Z44 suffers both from character coding and scoring errors (see under Respiratory pigments for discussion). Consequently, the support of this character for a monophyletic clade of nemerteans and other coelomate protostomes is dubious.

The brain of adult nemerteans develops close to the apical organ only in direct developing nemerteans, whereas the apical organ in pilidium larvae takes no part in the development of the adult central nervous system. This could justify the scoring of $\mathrm{Z145}$, but it depends on the interpretation that developmental stages that are part of the life cycle with direct development, which is presumed primitive for the phylum (Ax, 1995; Haszprunar et al., 1995; this paper), are in fact comparable to larvae. Z145 is misscored for several terminal taxa, and the observed scoring does not accurately reflect the adopted character definition. See under Adult brain derived from or associated with larval apical organ for a complete discussion.

As is taken up under Serial repetition of nerve collaterals, the phylogenetic significance of Z236 remains uncertain. Furthermore, various taxa have been misscored, and the character scoring covers a broad range of different morphologies, which creates doubt about the primary homology of this character. 
Table 3. Summary of alternative sister taxa for Nemertea with diagnostic synapomorphies and comments. See text for discussion.

\begin{tabular}{|c|c|}
\hline Sister taxon & Synapomorphies and source \\
\hline Platyhelminthes & see Table 2 \\
\hline Annelida & $\begin{array}{l}\text { closed blood vessels (Cavalier-Smith, 1998) } \\
\text { ciliated larvae without bivalved shells } \\
\text { (Cavalier-Smith, 1998) } \\
\text { two ventrolateral or one primitively paired ventral nerve } \\
\text { cord (Cavalier-Smith, 1998) }\end{array}$ \\
\hline
\end{tabular}

\section{Entoprocta Lobatocerebro- morpha}

'Teloblastica"'

\section{'Neotrochozoa'}

frontal gland system (ZI56)

gut with anus (Ax, 1989, 1995; B10b; RI5)

multiciliated epidermal cells (Ax, 1995)

schizocoel (B19; RI2)

mesoderm (from mesentoblast) gives rise in part to closed circulatory system of vessels only (B60a)

segmented or serial structures derived
ventral longitudinal nerve cord (ZII96)
gliointerstitial system (ZII98)

entomesoblast proliferation contributing to mesoderm (E7)

4d endomesoderm (P38)

mesodermal germ bands derived from 4d (P39)

lateral coelom derived from mesodermal bands (P40)

ventral nervous system (P103)

gliointerstitial cell system (P110)

schizocoelous formation of body cavity lined with mesodermal peritoneum (E17)

swimming/feeding band(s) of cilia in larvae with compound cilia (E39; H5)

pelagic larvae with apical ciliary tuft and plate (E41) cerebral rhabdomeric larval ocelli or integumentary pigment cups (E43)

\section{1}

dermal circular (or external transverse) muscular fibers (E131)

blood pigments $(\mathrm{H} 24 ; \mathrm{Z} 44)$

only testis sacular $\rightarrow$ both testis and ovarium sacular (H31)

coelom as defined histologically (Z30)

gonocoel (Z31)

coelomocytes (Z33)

podocytes (Z43)

adult brain derived from/associated with larval apical

organ/apical pole (Z145)

serial repetition in nerve collaterals (Z236)

\section{Comments}

not homologous

domain of definition not fulfilled: widespread in Metazoa

domain of definition not fulfilled:

widespread in protostomes

Should also be scored as present for Cycliophora

domain of definition not fulfilled, convergent

domain of definition not fulfilled: widespread in Metazoa

ok

nemertean blood vessels are not homologous with those of other bilaterians

) homology not supported

many phyla are misscored

various rescorings possible

ok, but in conflict with improperly scored E5

ok but convergent

ok (unique)

ok (unique), but status as independ ent character from P38 questionable spurious character coding and scoring

various rescorings possible various rescorings possible, and character definition is overly restrictive

dependent upon unsupported presence of pilidium larvae in nemertean ground pattern

ok

not a genuine larval character in nemerteans: only present in juveniles of direct developing nemerteans and post-metamorphic stages of indirect developers various misscorings and domain of definition not fulfilled unjustified character coding and various scoring problems domain of definition not fulfilled and scoring error for rhabditophorans various misscorings problematic scoring problematic scoring possibly ok, but various misscorings problematic scoring

various misscorings and uncertainty about primary homology

\footnotetext{
' The exact composition of this clade varies around a core of Sipuncula, Echiura, Annelida, Mollusca, and Arthropoda. See discussions of individual studies for details.

${ }^{2}$ The exact composition of this clade varies around a core of Sipuncula, Echiura, Annelida, and Mollusca. See discussions of individual studies for details.
} 


\section{Neotrochozoa (plus Myzostomida, Lobatocerebrum, and Jennaria)}

Zrzavý (2003)

- ventral longitudinal nerve cord (ZII96)

- gliointerstitial system (ZII98)

\section{Comments}

The possession of a ventral longitudinal nerve cord is an ambiguous synapomorphy of the nemerteans, neotrochozoans, myzostomids, Lobatocerebrum, and Jennaria. However, there are several problems with the scoring of this character. Sørensen et al. (2000) (S54) and Nielsen (2001) (NI46) are cited as sources for this character, and the three studies exhibit identical character scorings for taxa shared between them. However, the character definition adopted in Sørensen et al. (2000) and Nielsen (2001) differs from the one adopted in Zrzavý (2003). Sørensen et al. (2000) and Nielsen (2001) present a more restricted character definition, namely ventral nerve cords that are paired or secondarily fused during ontogeny. Consequently, the less restrictive definition used in Zrzavý (2003) should be reflected in a revised scoring. This is not the case. Many phyla are misscored as lacking ventral nerve cords. These include the hemichordates (based upon enteropneust anatomy which is considered representative of the hemichordate ground pattern in Zrzavý's analysis), cycliophorans (all motile stages of the life cycle have ventral nerve cords, Funch, 1996; Funch \& Kristensen, 1997; Obst \& Funch, in press), and micrognathozoans (Kristensen \& Funch, 2000).

Moreover, it is unclear why taxa such as the gnathostomulds, rotifers, platyhelminths, and nemerteans are scored as "?." These taxa do possess ventral or ventrolateral nerve cords (Clément \& Wurdak, 1991; Lammert, 1991; Rieger et al., 1991b; Turbeville, 1991). Although Zrzavý (2003) did not supply any justification for his scoring, the fact that Nielsen (2001) is cited as a source may indicate that Zrzavý (2003) accepts Nielsen's conclusion that at least the platyhelminths and nemerteans lack a ventral nervous system. However, the basis for this reasoning is highly questionable (see discussion under Alternative phylogenetic hypotheses for Platyhelminthes). Similarly, phyla such as Nema- toda, Nematomorpha and Priapulida with unpaired ventral nerve cords should be rescored for the restrictive characters in Sørensen et al. (2000) (S54) and Nielsen (2001) (NI46).

The possession of a gliointerstitial cell system is optimized as an unambiguous autapomorphy of the clade in question. However, the distribution of this character should be carefully reassessed as available evidence indicates the presence of this feature in taxa typically scored as lacking it (see discussion under Gliointerstitial cell system).

\section{Neotrochozoa}

Peterson \& Eernisse (2001)

- 4d endomesoderm (P38)

- mesodermal germ bands derived from 4d (P39)

- lateral coelom derived from mesodermal bands (P40)

- ventral nervous system (P103)

- gliointerstitial cell system (P110)

\section{Comments}

The interpretation of $4 \mathrm{~d}$ endomesoderm as a synapomorphy of the nemerteans and the neotrochozoans could in this case imply convergence with the rhabditophoran platyhelminths. However, it should be noted that for most of the taxa interposed between rhabditophorans and Nemertea + Neotrochozoa the cellular source of the mesoderm remains unknown (Cycliophora, Gnathostomulida, Ectoprocta). Thus, the overall homology of $4 \mathrm{~d}$ mesoderm cannot yet be discounted completely, which would remove this character as a synapomorphy of $\mathrm{Ne}$ mertea + Neotrochozoa. Current information, however, supports $\mathrm{P} 38$ as a convergent synapomorphy of Nemertea + Neotrochozoa.

The presence of mesodermal bandlets derived from $4 d$ (P39) may be a genuine synapomorphy of the nemerteans (Henry \& Martindale, 1998a) and neotrochozoans. Mesoderm bands appear to be absent in Platyhelminthes, as is also suggested by a recent cell tracing study of the polyclad Hoploplana inquilina (Boyer et al., 1998). The value of $\mathrm{P} 40$ as an independent character from P39 is questionable, because these bands of mesoderm later develop into 
schizocoels. Because P40 does not refer to the cellular source of the mesodermal bands, however, the onychophorans and arthropods could be scored as well in contrast to the scoring of $\mathrm{P} 40$, but fresh investigations on the embryology of these latter phyla are needed.

The analysis of Peterson \& Eernisse (2001) unequivocally (ACCTRAN optimization) suggests three independent origins of a ventral nervous system (P103): at the base of a clade (Nemertea Neotrochozoa), at the base of a clade (Rotifera Gnathostomulida Cycliophora Platyhelminthes), and at the base of a clade (Gastrotricha Chaetognatha Ecdysozoa). It is very doubtful whether this pattern of character transformations is a reliable reflection of nervous system evolution. The character scoring falsely suggests that the nervous system configurations observed in all other taxa, ranging from the poriferans and placozoans to the chordates and phoronids, are representative of a clear alternative character state, namely the lack of a ventral nervous system. Clearly, this is not the case. To unite the morphology of the placozoans (lacking a nervous system) and chordates (with a complex nervous system) in a common character state that contrasts with the ventral nervous system of the gastroneuralians is obviously not straightforward (see Jenner, 2002 for a general discussion of coding problems in metazoan cladistics). Furthermore, the hypothesis of three independent origins of a ventral nervous system is based on the distribution of the phoronids, brachiopods, ectoprocts, and entoprocts among the taxa with a ventral nervous system. Disregarding the ventral location of the entoproct central ganglion, which could justify a revised scoring for P103, it is at least equally likely that these taxa have modified their nervous systems concomitant with their adult sessility, which is consistent with the single origin of a ventral nervous system as supported by Nielsen (2001: NI46), and Zrzavý et al. (1998: Z234).

As is argued under Gliointerstitial cell system, the phylogenetic significance of this feature remains to be carefully assessed, as it may either be a homoplasy or a symplesiomorphy at this phylogenetic level.

\section{Placing Nemertea: comparison of alternative hypotheses}

The hypothesis that Nemertea and Annelida are sister groups proposed by Cavalier-Smith (1998) can be rejected. None of the three proposed synapomorphies can be upheld. Closed blood vessels are not homologous between the nemerteans and the annelids, and ciliated larvae and ventral or ventrolateral nerve cords are widespread among metazoans, and most likely plesiomorphic on this level. Although none of the included analyses yielded support of a sister group relationship between nemerteans and molluscs, the matrices of both Meglitsch \& Schram (1991) and Zrzavý et al. (1998) included a potential synapomorphy of this grouping. This character is discussed under Prototrochal lobes.

As is discussed under Platyhelminthes, the character support for a sister group relationship between the nemerteans and platyhelminths is mainly dependent upon the unsupported postulation of a life cycle with pilidium larvae in the ground pattern of Nemertea. The most extreme illustration of character selection differences between studies can be found, as discussed before, for the analyses of Nielsen $(1995,2001)$, Nielsen et al. (1996) and Sørensen et al. (2000), for which with the exception of a single character, all synapomorphies of a clade Parenchymia (Platyhelminthes and Nemertea) are unique to these studies. While not all studies include the same set of characters, it can nevertheless be concluded that most studies include one or several characters for source of mesoderm and mode of coelom formation, which generally support a close relationship of the nemerteans with the neotrochozoans, with or without the panarthropods. Turbeville (2002) showed that adding two new characters (schizocoel and gliointerstitial cell system) to the data matrix of Nielsen (2001) led to the collapse of the sister group relationship between the platyhelminths and the nemerteans, and to the placement of the latter as a sister phylum to the coelomate protostomes.

So far the most convincing synapomorphies that may be unique for the nemerteans and neotrochozoans or teloblasticans (plus or minus panarthropods) are the presence of mesodermal bands derived from $4 \mathrm{~d}$, and a schizocoel. Various other features may 
strengthen the nexus of the nemerteans and neotrochozoans or teloblasticans (schizocoelians) such as the presence of a coelom, '4d-mesoderm (not in panarthropods), and podocytes, but so far these features have been indicated to be convergent. Some of the most problematic characters that supposedly unite nemerteans with other phyla are the presence of respiratory pigments (Haszprunar, 1996a; Zrzavý et al., 1998) and a gonocoel (Zrzavý et al., 1998), the coding and scoring of which should be thoroughly reassessed before they can profitably be used again in a cladistic analysis.

The sister group relationship suggested by Zrzavý et al. (2001) is supported by one convergent character that could also be scored for Cycliophora. However, because the clade (Nemertea Entoprocta Lobatocerebrum) is placed as a sister group to a clade Neotrochozoa, this hypothesis is closely similar to the two remaining hypotheses that propose a sister group relationship with either a clade minimally composed of Neotrochozoa + (Pan)Arthropoda, known as Teloblastica or Schizocoelia, or Neotrochozoa alone. Whether nemerteans are strictly closely related to neotrochozoans or teloblasticans is dependent upon the resolution of the phylogenetic significance of characters that will not directly influence the placement of nemerteans, e.g., those uniting annelids and arthropods (see Nielsen, 2001 and Wägele \& Misof, 2001 for a discussion of these characteristics). The discussion of these features is outside the scope of this paper, but it must be noted that many of the characters that are marshaled in support of either the Teloblastica or Neotrochozoa hypothesis are in fact unable to distinguish between these two alternatives because these features are present in at least some panarthropods as well. This can be concluded, for example, for a gut with anus, a schizocoel, a histologically defined coelom, lateral coeloms, segmented mesodermal organs, ventral nervous system, gonad organization, podocytes, dermal circular muscles, and serially repeated nerve collaterals. The characters uniquely supporting a close affinity of Nemertea with Neotrochozoa (excluding panarthropods) are mainly those related to the presence of an entomesoblast and derived mesodermal structures (including coeloms), and various larval characters, such as an adult brain derived from, or associated with a larval apical organ (see discussion in Part II). However, as is discussed elsewhere in this paper, the phylogenetic value of most larval characters hinges on the unsupported assumption that pilidium larvae are part of the nemertean ground pattern.

Irrespective of the placement of the panarthropods, nemerteans group together with the neotrochozoans. This leads to an encouraging conclusion: the close relationship between the nemerteans and at least the neotrochozoans is currently the best defended hypothesis despite the many identified scoring problems, and it represents a consensus among all comprehensive morphological cladistic analyses.

\section{Placing Nemertea in molecular and total evidence analyses}

Analyses of $18 \mathrm{~S}$ rDNA sequences, intermediate filament proteins, and recently also myosin II sequences have not unambiguously identified a nemertean sister taxon (uncertain affinities to different neotrochozoans, entoprocts, brachiopods, cycliophorans, or rotifers are suggested), but their protostomian and lophotrochozoan affinity is generally supported (Eernisse, 1997; Erber et al., 1998; RuizTrillo et al., 1999, 2002; Giribet et al., 2000; Zrzavý et al., 1998, 2001; Peterson \& Eernisse, 2001; Jondelius et al., 2002). Until recently, monophyly of the phylum has been difficult to support in some studies (Winnepenninckx et al., 1998; Giribet et al., 2000), and even with a broader species sampling and the use of a restricted number of outgroup taxa, 18S rDNA data only weakly support the monophyly of Nemertea (Sundberg et al., 2001). Thollesson \& Norenburg (2003) provided the first robust molecular evidence for nemertean monophyly.

Total evidence analyses of morphological and $18 \mathrm{~S}$ rDNA data yield a clearer signal: nemerteans are either the sister group to the neotrochozoans, together comprising a clade Eutrochozoa (Zrzavý et al., 1998; Giribet et al., 2000; Peterson \& Eernisse, 2001 ), or they are closely related to the neotrochozoans plus Cycliophora, Entoprocta, and Lobatocerebrum (Zrzavý, 2003). In apparent conflict with these findings is the sister group relationship of the nemerteans and sipunculans purported in the 
total evidence analyses of Zrzavý et al. (2001), a topology that also receives some support from molecular data alone, but not from morphological data alone.

In conclusion, molecular data does not unambiguously support any of the sister group hypotheses based on morphological data, a situation that we already encountered with Platyhelminthes. On the other hand, most total evidence analyses provide support for a close relationship between Nemertea and Neotrochozoa, which is also suggested by most comprehensive morphological studies (Eernisse et al., 1992; Peterson \& Eernisse, 2001; Haszprunar, 1996a). However, in view of the ambiguous phylogenetic signal inherent in $18 \mathrm{~S}$ data alone, it is likely that this topology in total evidence analyses is mainly the result of the morphological signal. Morphological data matrices that instead support the monophyly of Teloblastica (Neotrochozoa + Panarthropoda) (Nielsen et al., 1996; Nielsen, 2001; Sørensen et al., 2000) have not yet been combined with $18 \mathrm{~S}$ sequence data.

\section{Alternative Phylogenetic Hypotheses for Gnathostomulida}

The history of initial phylogenetic speculations about Gnathostomulida are reviewed elsewhere (Sterrer et al., 1985; Kristensen \& Funch, 2000; Sørensen, 2002). Sørensen (2001) performed the first cladistic analysis of gnathostomulid genera. During the last decade at least five different sister group hypotheses have been defended on the basis of morphological cladistic analyses. Most prominent among these are the Plathelminthomorpha Hypothesis and the Gnathifera Hypothesis. According to the Plathelminthomorpha Hypothesis, Gnathostomulida and Platyhelminthes are sister taxa, while the Gnathifera Hypothesis supports a sister group relationship between Gnathostomulida and Syndermata (+ Micrognathozoa: Kristensen \& Funch 2000; Sørensen et al., 2000; Sørensen, 2001). The Plathelminthomorpha Hypothesis merits special consideration, first because it was forcefully championed by Ax (1985, $1989,1995)$ who described the group in the mid $1950 \mathrm{~s}$, and second because it is supported by most comprehensive morphological cladistic analyses published to date. The Gnathifera Hypothesis warrants special attention because despite the apparently superior quality of its supporting characters (relative to the plathelminthomorphan apomorphies), just two computer-assisted cladistic analyses (Sørensen et al., 2000 and Nielsen, 2001) support gnathiferan monophyly.

\section{(Gnathostomulida Platyhelminthes)}

See under Platyhelminthes.

\section{(Gnathostomulida Gastrotricha)}

Zrzavý et al. (1998) named this clade found in their total evidence analysis Neotrichozoa, and Cavalier-Smith (1998) named it Monokonta, in both cases based upon the secondary acquisition of partially or completely monociliary epithelia. Because Cavalier-Smith (1998) did not attempt to base his classification on the phylogenetic distribution of characters, his conclusions will not be discussed further here. Proposed synapomorphies: Zrzavý et al. (2001). See figure 4.

Zrzavý et al. (2001)

- loss of multiple protonephridial terminal cells (ZI30)

- protonephridial filter formed by weir-like fenestrations of the terminal cell's wall (ZI32)

(- serial protonephridia (ZI34)

\section{Comments}

Bartolomaeus \& Ax (1992) and Ax (1995) postulate the presence of a single protonephridial terminal cell in the ground pattern of the Bilateria, a conclusion not necessarily supported by the phylogenetic distribution of protonephridial types. For example, the analysis by Zrzavý et al. (2001) suggests that multiple terminal cells (ZI30) are plesiomorphic and that single terminal cells have evolved independently four times: in Gastrotricha + Gnathostomulida, Entoprocta + Lobatocerebrum, Mollusca, and Cycliophora. All other taxa either lack protonephridia or possess more than one protonephridial 
terminal cell. Several comments on character scoring are in order.

First, for a logically consistent character scoring it is critical to determine the comparable semaphoronts. The observed scoring suggests that both adults and larvae are used, but not consistently. For example, for the Mollusca and the Cycliophora the larval ground patterns are scored (single terminal cells; adult ground patterns lack protonephridia). However, for the Annelida only the adult morphology is scored (multiple terminal cells), despite the proposal of protonephridia with single terminal cells (head kidneys) in the ground pattern of annelid trochophore larvae (Bartolomaeus, 1993b, 1995, 1998; there is variation in the number of terminal protonephridial cells in different annelid larvae). Also, the Rhabditophora is scored as having multiple protonephridial terminal cells despite the demonstration of protonephridia with single terminal cells in polyclad Götte's larvae (Rohde, 2001), but this scoring is justified because Götte's larvae are not likely to be informative for the rhabditophoran ground pattern. Because ultrastructural studies of larval nephridia are not available for all taxa, character scoring is likely to change in future cladistic analyses.

Second, the scoring of single terminal cells for Entoprocta is based upon old data (Bartolomaeus $\&$ Ax, 1992). Newer studies indicate the presence of two terminal cells in the adult protonephridia (Franke, 1993; Nielsen \& Jespersen, 1997).

Third, although gastrotrich protonephridia are generally considered to be representative of the archetypal bilaterian type (Bartolomaeus \& Ax, 1992; $A x, 1995)$, and are scored accordingly for ZI30, protonephridia with more than one terminal cell are not rare within the phylum (Ruppert, 1991b). Therefore, pending further studies, the current scoring may be retained, but the exact phylogenetic distribution of different protonephridial types within Gastrotricha will eventually have to show whether this is justified or not.

Mapping the distribution of protonephridial types on the morphological trees of four recent cladistic analyses (Sørensen et al., 2000; Nielsen, 2001; Peterson \& Eernisse, 2001; Zrzavý et al., 2001) leaves the plesiomorphic number of protonephridial terminal cells uncertain. In contrast, the analysis of Zrzavý (2003) supports monociliate terminal cells as the plesiomorphic design for the Bilateria. It should be noted that protonephridia with single terminal cells appear to be restricted to small animals, such as larval molluscs, annelids, and cycliophorans. Smith \& Ruppert (1988) pointed out that within Annelida, large bodied adults appear to have multiple terminal cells while interstitial forms have single terminal cells (see also Westheide, 1986 for hints for a correlation of body size and nephridial architecture in polychaetes). Although it is very difficult to assess the value of such functional considerations for the phylogenetic significance of characters, the existence of these possible correlations may help to rationalize inferred character transformations.

Zrzavý et al. (2001) (ZI32) scored the presence of terminal cell fenestrations (pores or slits) that form a protonephridial.filter in five taxa: Gastrotricha, Gnathostomulida, Seison, Catenulida, and Myzostomida. A careful study of protonephridial ultrastructure shows that this scoring is inaccurate. Terminal cell fenestrations are additionally known from monogonont and bdelloid rotifers (in addition to seisonids), Phoronida, Platyhelminths (in addition to catenulids), Nemertea, Mollusca (larvae), Annelida (larvae, but they have also been reported from several adult interstitial polychaetes), Entoprocta, Kinorhyncha, Echiura (male Bonellia), and probably Acanthocephala (Bartolomaeus, 1985, 1989b, 1995, 1998; Bartolomaeus \& Ax, 1992; Schuchert, 1990; Clément \& Wurdak, 1991; Dunagan \& Miller, 1991; Rieger et al., 1991b; Turbeville, 1991; Bartolomaeus \& Ax, 1992; Smith, 1992; Ahlrichs, 1993a, 1995; Haszprunar \& Ruthensteiner, 2000; Rohde, 2001; Ruthensteiner et al., 2001).

It is clear that preciously little morphological study underlies the scoring of this character in Zrzavý et al. (2001) (and the identical scoring of ZII69 in Zrzavý, 2003, see below). Bartolomaeus \& Ax (1992) and Bartolomaeus (1995) hypothesized that terminal cell fenestrations, which may occur in different arrangements, are part of the plesiomorphic design of protonephridia. Accordingly, rescoring of ZI32 is necessary for any proper interpretation of the phylogenetic significance of fenestrated protonephridial terminal cells. At present, independent evolution of at least some of these instances 
appear probable, for example, the protonephridium in the dwarf male of the echiuran Bonellia (adult echiurans typically have metanephridia). However, an electronmicroscopical study of larval protonephridia in the echiurans is needed to exclude the possibility that the male of Bonellia has retained the larval protonephridia.

The presence of serially repeated protonephridia (ZI34) is not a compelling synapomorphy for Gnathostomulida + Gastrotricha because the latter is scored as '?.' Serial protonephridia are furthermore scored as present in the Annelida, Myzostomida, and Lobatocerebrum. The presence of serial protonephridia in the ground pattern of the Annelida is doubtful. A single pair of larval head kidneys may be assumed primitive for the annelids (Bartolomaeus, 1998, 1999), but the presence of serial protonephridia in adult polychaetes is uncertain. Bartolomaeus (1999) revised his earlier conclusion that segmental protonephridia are an annelid ground pattern feature (Bartolomaeus \& Ax, 1992), and concluded instead that their occurrence in certain taxa is secondary (in agreement with the cladistic analysis of polychaete relationships by Rouse \& Fauchald, 1997). Although the Micrognathozoa was not included in the analysis they possess two pairs of protonephridia (Kristensen \& Funch, 2000).

Taken together, these three characters do not unambiguously support the monophyly of a clade Gnathostomulida + Gastrotricha. Even accepting the scoring of these characters in Zrzavý et al. (2001), this sister group hypothesis is sensitive to the coding of one particular complex character, ZI 21 , which codes for the presence of a molted cuticle with epicuticle, exocuticle, and endocuticle, with sclerotization. I performed a character coding experiment in which this complex character was partitioned into several arguably independent characters (Jenner, 2002). This character decomposition allows cuticular similarities uniquely shared between the gastrotrichs and the introvertans or ecdysozoans, but not the gnathostomulids, to attain potential phylogenetic significance that would otherwise remain hidden in the larger character complex. This recoding also resulted in the collapse of the clade (Gastrotricha + Gnathostomulida).

Because the analysis of Zrzavý et al. (2001) places the clade of Gastrotricha and Gnathostomulida as a sister group to all the remaining protostomes, except the phoronids and brachiopods, it is instructive to evaluate whether the characters that support this clade compellingly separate the gnathostomulids and gastrotrichs from all other protostomes. The pertinent characters are presence of multiciliate cells (ZI16), multiciliate protonephridial terminal cells (ZI31), and adult brain derived from/associated with larval apical organ (ZI45). The large protostome sister clade to the Gnathostomulida + Gastrotricha is composed of two sister clades: Ecdysozoa + Chaetognatha (for convenience called clade $A$ ), and a clade of the remaining protostomes (clade B) (see figure 4d). I will argue that none of these characters is convincing in separating Gnathostomulida + Gastrotricha from the remaining protostomes.

It is not immediately clear whether ZI16 is only scored for ectodermal cells (as is hinted by Zrzavý et al's defense for the scoring of chaetognaths), or also for other parts of the body. ZI16 is scored as '?' for all members of clade A, except Onychophora, which is scored as possessing multiciliate cells based on their presence in the metanephridia. If only ectodermal cells are to be scored then Onychophora should be scored as possessing monociliate cells only, because Bartolomaeus \& Ruhberg (1999) clearly indicated the presence of monociliate epidermal cells (albeit rudimentary) in the developing embryo. This would shift the transformation of ZI16 towards the branch supporting clade B. In contrast, if multiciliation is scored across other tissues, e.g., generally for epithelia, than the onychophorans should be rescored as polymorphic since their developing coeloms are lined by cells with single rudimentary cilia (Bartolomaeus \& Ruhberg, 1999). Although the deuterostomes are unequivocally scored as lacking multiciliate cells, these are also found in the enteropneusts and chordates, and additionally in the chaetognaths (Shinn, 1997; Nielsen, 2001). Moreover, the evolutionary origin of multiciliate cells within the Metazoa cannot be properly assessed when the non-bilaterians are excluded from the analysis, with multiciliate cells being typical for the ctenophores. These amendments increase the likelihood of the origin of multiciliate cells at the base of Acrosomata, and thus their plesiomorphy on the level of clade $\mathrm{A}$ and 
B. This is in agreement with all comprehensive morphological cladistic analyses that included a character on cell ciliation in their matrices: Nielsen et al. (1996), Nielsen (2001), Sørensen et al. (2000), Peterson \& Eernisse (2001), and Zrzavý (2003) (characters N8, NI11, S12, P13, ZII25). We should finally note that the gastrotrichs are scored as '?' for ZI16. Two recent cladistic analyses of internal gastrotrich relationships could be interpreted to support this scoring, because monociliate epidermal cells are present in the basal-most clades while multiciliate epidermal cells are prevalent in the other taxa (all chaetonotids except Neodasys and many macrodasyiids) (Hochberg \& Litvaitis, 2000, 2001a). See also the discussion under Multiciliate epidermal cells.

ZI31 is scored as 'inapplicable' for clade A because protonephridia are lacking in these phyla. Hence the support of ZI31 for monophyly of clade $\mathrm{A}+\mathrm{B}$ is equivocal.

ZI45 is scored as '?' for all members of clade A except Arthropoda. As is argued under Adult brain derived from or associated with larval apical organ, this scoring cannot be defended, and this character therefore represents a potential apomorphy only of clade B. In conclusion, the characters that separate a clade Gnathostomulida + Gastrotricha from the remaining protostomes are not compelling, which in turn creates serious doubt about the reliability of both the gnathostomulid sister group as well as their overall position within the Metazoa in the analysis of Zrzavý et al. (2001).

\section{(Gnathostomulida Syndermata)}

This clade is known as Gnathifera. Proposed synapomorphies: Ahlrichs (1995, 1997; followed by Garey et al., 1998); Haszprunar (1996b); Melone et al. (1998); Kristensen \& Funch (2000); Sørensen et al. (2000); Sørensen (2001); Herlyn \& Ehlers (1997); Nielsen (2001); Zrzavý (2003). When Kristensen \& Funch (2000) described Limnognathia maerski (Micrognathozoa), they proposed a possible sister group relationship between (Syndermata plus Micrognathozoa) and the gnathostomulids. The computer-assisted cladistic analyses of Sørensen et al. (2000), and Zrzavý (2003) supported this grouping. See figures $1,3,4$, and 5 .
Ahlrichs (1995) (A15)

- z-elements of muscles present as isolated z-dots

- cross-striated jaw or mastax muscles

- protonephridial canal cell surrounds lumen completely

- buccal ganglion (integrated into pharynx?)

- nervus pharyngeus

- pair of caudal ganglia

- pharynx as ventral evagination of foregut

- pharyngeal cuticular hard parts secreted by pharynx epithelium

- cuticular hard parts connected across secreting epithelium by cross-striated muscles

- esophagus present

- gland characters: frontal glands, buccal or salivary glands

\section{Comments}

The Z-elements of striated muscles indicate the position where one sarcomere separates from the next. These elements come in a variety of forms, including discs, dots and lines, and their morphology may vary within phyla and between different muscles within a single individual, e.g., Gnathostomulida, Lammert (1991), and the gastropods, Voltzow (1994). Although the presence of Z-dots may be a synapomorphy of Gnathifera, it should be noted that similar structures are widespread within the Metazoa, including possibly closely related taxa, such as the kinorhynchs and gastrotrichs (Kristensen \& Higgins, 1991; Ruppert, 1991b). A comprehensive cladistic analysis including this character is necessary to evaluate its overall phylogenetic significance across the Metazoa.

Protonephridia with an intracellular canal lumen could be a genuine synapomorphy of the rotifers and gnathostomulids, but the only two computerassisted cladistic analyses that included this character (Zrzavý et al., 1998: Z52; Peterson \& Eernisse, 2001: P68) do not support this interpretation (see also under Protonephridia with channel cell completely surrounding lumen).

Ahlrichs (1995) proposed that the mastax ganglion found in Rotifera and the buccal ganglion of Gnathostomulida may be homologous and serve as a synapomorphy for Gnathifera. Haszprunar (1996b) and Melone et al. (1998) (characters HaVIIIa and 
Me11, respectively), as well as Nielsen (2001: 310) followed this reasoning, but a recent investigation by Herlyn \& Ehlers (1997) removed the basis for these conclusions. They could not confirm the presence of a buccal ganglion in Gnathostomulida, and they concluded after reinterpreting earlier studies that "the Gnathostomulida lack any buccal ganglion" (Herlyn \& Ehlers, 1997: 144). This removes one potential autapomorphy of Gnathifera (it also weakens the support for Nielsen's expanded Gnathifera that includes chaetognaths). This same holds true for the nervus pharyngeus or buccal nerves that connect the cerebral ganglion and mastax ganglion in rotifers. Herlyn \& Ehlers (1997) could not confirm the presence of comparable nerves in Gnathostomulida. Interestingly, a pair of buccal nerves, but not a buccal ganglion was observed for the probably closely related Limnognathia by Kristensen \& Funch (2000).

The presence of caudal ganglia is unconvincing as a synapomorphy for the Gnathostomulida and Syndermata because caudal ganglia are distributed more widely throughout the Metazoa (not found in Limnognathia), including the potentially closely related cephalorhynchs and nematodes (Kristensen, 1991 a; Kristensen \& Higgins, 1991; Storch, 1991; Wright, 1991). Consequently, a wider consideration of caudal ganglia across the Metazoa is required for a proper assessment of their phylogenetic value. Two phylogenetic analyses by Zrzavý et al. (1998, 2001 ) included this character and a comprehensive sampling of phyla, but they scored Rotifera only (1998), or Rotifera and Gnathostomulida (2001). Without providing a clear definition of a caudal ganglion that strictly applies to these taxa, this scoring is merely arbitrary. Information on the organs or tissues that are innervated from the caudal ganglia may be a first step in the construction of a meaningful character.

A broader set of taxa has to be considered to assess the phylogenetic value of an esophagus as well as the gland characters used to unite the gnathostomulids and the syndermates.

The remaining characters are also variously included in later computer-assisted cladistic analyses, and because they are part of one structural and functional complex, the muscular pharynx with its hard parts, they will be discussed together here.
The two principal characters that merit discussion are:

1) the presence of jaw elements with tube-like support rods composed of electron lucent material surrounding an electron-dense core (Ah1, HaVIIIa, Me18, K1, S42, NI34, G7, P90, L57, Z66, ZI26, Wa25, ZII52)

2) the presence of cross-striated pharyngeal muscles that attach to the jaw elements through epithelial cells (S43, NI35, ZII52)

The study of the trophi of the rotifer Seison by Rieger \& Tyler (1995) has refocused the attention upon the structural similarities of rotifer trophi and gnathostomulid jaw elements to which potential phylogenetic relevance had already been assigned in earlier studies (see Rieger \& Tyler, 1995 for literature). More recent transmission electron microscopical studies of gnathiferan (including micrognathozoan) jaw elements have confirmed their unique morphology found nowhere else within the Metazoa (Herlyn \& Ehlers, 1997; Kristensen \& Funch, 2000; Sørensen, 2000). Interestingly, the discovery of Micrognathozoa has been one of the main motivations for reconsidering the similarities in jaw structure between the rotifers and gnathostomulids (Kristensen \& Funch, 2000). Before the complete description of Limnognathia was reported in the literature, it already started to play an important role in the phylogenetic discussions about gnathiferans (Kristensen, 1995: he called Limnognathila New group 1"; Ahlrichs, 1997: he called Limnognathia "New group A" followed by Herlyn \& Ehlers, 1997). On the basis of scanning electron microscopy, Sørensen (2000) and Sørensen \& Sterrer (2002) suggested additional unique similarities between rotifer and gnathostomulid jaw elements, and Kristensen \& Funch (2000) proposed further homologies between jaw elements in rotifers, gnathostomulids, and Limnognathia, but these proposals have not yet been included in cladistic data matrices.

Despite the unique ultrastructural similarities of gnathiferan jaw elements, the inclusion of this character in a computer-assisted cladistic analysis does not guarantee a monophyletic Gnathifera, as is il- 
lustrated by Wallace et al. (1996), Zrzavý et al. (1998, 2001), Giribet et al. (2000), and Peterson \& Eernisse (2001). Interestingly, the only cladistic analyses with a sufficiently broad taxon sampling that found a monophyletic Gnathifera supported by the presence of this character are Sørensen et al. (2000) and Nielsen (2001). These studies incidentally are also the only ones that include a separate character on the mode of pharyngeal muscle attachment [ZII52 in Zrzavý (2003) combines the presence of pharyngeal hard parts and the type of pharyngeal muscle attachment]. Whether this coding of two separate characters rather than one causes this difference in topology is difficult to assess intuitively because the data matrices of Sørensen et al. (2000) and Nielsen (2001) are very similar, while they differ greatly from the data matrices of the other studies. I therefore performed two experiments.

In the first experiment, I analyzed the original data matrix of Nielsen (2001) (heuristic search, 100 random addition replicates, TBR branch swapping, excluding character 64 as Nielsen did for his strict consensus), and then I re-analyzed the matrix while excluding character NI35 that codes for the mode of pharyngeal muscle attachment. These two analyses yielded exactly the same strict consensus of the same four MPTs with a monophyletic Gnathifera, a situation identical to the analysis and results (fig. 56.1 ) of Nielsen (2001). The tree statistics changed so little between the analyses that they are not considered here.

In the second experiment, I re-analyzed the original data matrix of Peterson \& Eernisse (2001) [same analysis parameters as in the first experiment], and subsequently I introduced an extra character into their matrix coding for the pharyngeal muscle attachment type found in gnathiferans and scored accordingly. The first analysis yielded the 20 MPTs and well-resolved strict consensus found by Peterson $\&$ Eernisse (2001: fig. 1). In sharp contrast, the reanalysis with the second potential gnathiferan autapomorphy resulted in a dramatic collapse of the strict consensus tree, leaving one huge polytomy for Bilateria. The only clades that were retained are Ecdysozoa, Eutrochozoa, Deuterostomia, Brachiopoda + Phoronida and Platyhelminthes. The relationships between these and all other bilaterian phyla remained totally unresolved. In view of the low bootstrap supports for large portions of Peterson \& Eernisse's strict consensus, this result is not entirely surprising. Furthermore, Gnathostomulida no longer formed the sister group of Platyhelminthes, and its position and that of Rotifera remained entirely unresolved.

These experiments illustrate that the same change for two taxa in two different matrices can have entirely different effects, depending upon the differential presence or absence of conflicting phylogenetic signals and noise in these matrices. It also underlines the importance of character selection for determining the outcome of a cladistic analysis. Just adding one additional potential gnathiferan autapomorphy to the matrix of Peterson \& Eernisse collapses the Plathelminthomorpha together with most of the remaining Bilateria in an unexpected way. In this context, it becomes important to ask whether the separate coding of a character on the attachment of muscles to pharyngeal hard parts is justified, as is done in the matrices of Sørensen et al. (2000) and Nielsen (2001), but not in the other studies.

Cross-striated pharynx muscles that attach to the cuticular jaw elements are also found in the Micrognathozoa (Kristensen \& Funch, 2000). Naturally, when comparable pharyngeal hard parts are lacking in other taxa, they should logically be scored as 'inapplicable' for mode of pharyngeal muscle attachment, but neither Sørensen et al. (2000) nor Nielsen (2001) adopt this scoring. Sørensen et al. (2000) and Nielsen (2001) also score this feature as present in the Annelida. However, it is only found in the eunicid polychaetes, which are unlikely to be representative of the annelidan ground pattern (Rouse \& Fauchald, 1997). However, if we, nevertheless, choose to accept this scoring, we have to confront an interesting issue. Cross-striated body muscles (as opposed to cross-striated pharyngeal muscles) in taxa such as kinorhynchs, loriciferans, cycliophorans, and possibly nematodes (Wright, 1991, fig. 28) also do not attach directly to the cuticle, but through the intermediate of an epidermal cell (Kristensen \& Higgins, 1991; Funch \& Kristensen, 1997; Neuhaus et al., 1997b). Similarly, somatic muscles of the Micrognathozoa always attach through epidermal cells to the, in this case intracellular, skeletal plates that are located in the lateral 
and dorsal body regions (Kristensen \& Funch, 2000). In fact, a survey of muscle attachment types throughout the Metazoa reveals that the attachment of muscles to the cuticle through intermediate epithelial cells is much more widespread. It has, for example, been reported for arthropod muscles, tardigrade stylet muscles, cephalorhynch introvert retractor muscles (through tanycytes), the beak muscles in cephalopods (through beccublasts), gastrotrich muscles, cycliophoran muscles, ectoproct muscles (attachment to ectocyst), and chaetognath head muscles (Kristensen \& Higgins, 1991; Ruppert, 1991b; Mellon, 1992; Dewel et al., 1993; Budelmann et al., 1997; Funch \& Kristensen, 1997; Mukai et al., 1997; Shinn, 1997; Nielsen, 2001).

Recognizing the widespread distribution of this mode of muscle attachment is important for properly evaluating the phylogenetic significance of cross-striated muscle attachment to pharyngeal hard parts in gnathiferans, as it lessens the probability that this type of muscle attachment is a novel autapomorphy of Gnathifera independent from the presence of pharyngeal hard parts. This information can thus be used as an argument against coding a separate character for this mode of muscle attachment with a scoring restricted to gnathiferans (S43, NI35). This mode of muscle attachment may either be plesiomorphic at this level, or perhaps convergently evolved in different taxa, but further comparative ultrastructural studies are needed to elucidate this interesting issue.

Ahlrichs (1997)

- plates of parallel-layered cuticular rods building pharyngeal hard parts, jaws, which are embedded in a cuticular matrix (Ahl)

\section{Comments}

This character is discussed above.

\section{Haszprunar (1996b)}

- buccal apparatus with specific jaw, cross-striated muscles and buccal ganglion (HaVIIIa)

- caudal ganglion (HaVIIIb)

- protonephridial canal cell surrounds lumen completely (HaVIIIc)

- frontal (=praepharyngeal glands)? (HaVIIId)

\section{Comments}

These characters are discussed above.

Melone et al. (1998)

- pharyngeal ganglion (unpaired) present (Me11)

- masticatory apparatus: substructure tubular rods (Me18)

- photoreceptive eyespots absent (Me58)

\section{Comments}

The phylogenetic value of the absence of photoreceptive eyespots in the gnathostomulids and acanthocephalans as a gnathiferan autapomorphy needs to be carefully assessed through a comprehensive cladistic analysis that includes all applicable taxa. Furthermore, based on out-group comparison it must be concluded that the absence of eyespots is actually a plesiomorphy in the analysis of Melone et al. (1998), and thus phylogenetically uninformative at this level. The other two characters are discussed above.

Nielsen (2001)

- jaw-like structures with tubes composed of lucent material surrounding an electron-dense core (NI34)

- pharynx with cross-striated muscles, attached to jaws by epithelial cells (NI35)

- mouth region with chitinous membrane (NI63)

\section{Comments}

Nielsen (1995) tentatively regarded gnathostomulids as highly derived annelids, but Nielsen (2001) regarded them as a distinct taxon more closely associated with syndermates. NI63 is a modified version of N12 which coded for the presence of a mouth/ esophagus with a spiny/toothed membrane consisting of crystalline chitin, and both characters are scored present for Rotifera and Chaetognatha. Sørensen et al. (2000) argued against the scoring of N12 claiming that crystalline chitin has not been found in rotifers. In view of the adjusted character coding, the scoring of NI63 now appears to be justified, but a different problem is introduced. If all taxa with just a chitinous membrane (cuticle) in 
the mouth region are to be scored, several more taxa than just the rotifers and chaetognaths need to be scored as present, including the panarthropods and various cycloneuralians (Kleinow, 1993; Neuhaus et al. 1996; Neuhaus et al., 1997a; Lemburg, 1998; Schmidt-Rhaesa et al., 1998). Furthermore, as long as the presence of chitin has not been demonstrated in the gnathostomulids this character is not a convincing gnathiferan autapomorphy. The other two characters are considered above.

Syndermata plus Micrognathozoa

Kristensen \& Funch (2000)

- cuticular jaws with tubes composed of lucent material surrounding an electron-dense core (K1)

\section{Comments}

This character is discussed above.

Sørensen et al. (2000), Sørensen (2003)

- jaws of tubes composed of lucent material surrounding an electron-dense core (S42)

- pharynx with cross-striated muscles, attached to jaw elements by epithelial cells (S43)

- jaws with pincers caudally articulating into unpaired pedicle

\section{Comments}

S42 and S43 are discussed above. It should be noted that both S42 and S43 were scored as present for Chaetognatha and absent for Seisonida, but this is obviously a typing error in the text matrix (Table 1 in Sørensen et al., 2000; M. V. Sørensen, pers. comm.).

Based upon detailed comparative studies of the jaw apparatuses of the rotifers, gnathostomulids, and Limnognathia maerski Sørensen (2003) proposed a novel synapomorphy for gnathiferans based on the morphological similarities between the main jaws in $L$. maerski, the gnathostomulid lamellae symphysae, and the rotiferan rami.
Zrzavý (2003)

- jaws with tube-like support rods, attached to cross-striated pharyngeal muscles by epithelial cells (ZII52)

- extended non-contractile regions of the pharyngeal musculature (ZII59)

- mouth region with chitinous membrane (ZII60)

- protonephridial filter formed by weirlike fenestrations of the terminal cell wall (ZII69)

- protonephridial canal cell cilia absent (ZII70)

- loss of sperm acrosome (ZII79)

- sperm lacking accessory centriole (ZII83)

\section{Comments}

Character ZII52 is properly scored for the gnathiferans and Micrognathozoa. This character is further discussed above.

Character ZIII59 is a potential synapomorphy for a clade of the gnathiferans and Micrognathozoa, although this feature has not been observed in the latter.

Character ZII60 is scored as present for the chaetognaths and rotifers only, and is therefore no convincing synapomorphy of the clade in question. Furthermore, as is discussed above for character NI63, several additional phyla also possess a chitinous membrane in the mouth region, notably the panarthropods and various cycloneuralians. Accordingly, these phyla need to be rescored.

Although character ZII69 is optimized as an unambiguous synapomorphy for the clade in question, it is misscored for many phyla and should be thoroughly restudied. The scoring of this character is discussed above for character ZI32.

The lack of protonephridial canal cell cilia is an ambiguous synapomorphy of the clade in question, and ZII70 is scored only for the gnathostomulids and rotifers. At least one case of convergent loss of protonephridial canal cell cilia is known, viz. in the scaphopod molluscs (Ruthensteiner et al., 2001).

The loss of a sperm acrosome is an unambiguous (albeit convergent) synapomorphy of the clade in question. Althought I did not attempt to study the distribution of sperm acrosomes across the Metazoa, several comments on character scoring may nevertheless be made. Although the gnathostomulids are scored as lacking a sperm acrosome, one may 
be present in the tip of the sperm of filospermoid gnathostomulids (Sterrer et al., 1985, fig. 12.4a). The cycliophorans are scored as possessing a sperm acrosome, but this data is unconfirmed (Funch \& Kristensen, 1997; M. V. Sørensen personal communication).

Zrzavý (2003) scored the myzostomids, monogonont rotifers, Seison, and the acanthocephalans as having sperm without an accessory centriole. However, this scoring is inaccurate. Although I did not comprehensively study the distribution of this character across the Metazoa, sperm accessory centrioles are also lacking in the gastrotrichs, kinorhynchs, and nematomorphs (Ahlrichs, 1995; Schmidt-Rhaesa, 1999). Moreover, lack of an accessory centriole in sperm is probably correlated with the evolution of modified or filiform sperm, and this does not warrant the coding of an independent character. For example, the meiobenthic priapulid genus Tubiluchus possesses filiform sperm that probably evolved within the Priapulida (Storch et al., 2000), and it too is accompanied by the absence of an accessory centriole (see also discussion under Sperm without accessory centriole).

\section{(Gnathostomulida Cycloneuralia)}

Proposed synapomorphies: Wallace et al. (1996). See figure 7.

Wallace et al. (1996)

- smooth muscles absent (Wa11)

- pharynx shape triangular or star-shaped (Wa23)

\section{Cominents}

The absence of smooth muscles may unite the gnathostomulids with the cycloneuralians, but the restrictive taxon sampling in the analysis of Wallace et al. (1996) did not allow the domain of definition of Wall to be fulfilled. For example, the potentially closely related and recently discovered Micrognathozoa (Kristensen \& Funch, 2000), and probably also the Cycliophora (Funch \& Kristensen, 1997) lack smooth muscles. A survey of muscle ultrastructure across the Metazoa is necessary to fulfill the domain of definition of this character,

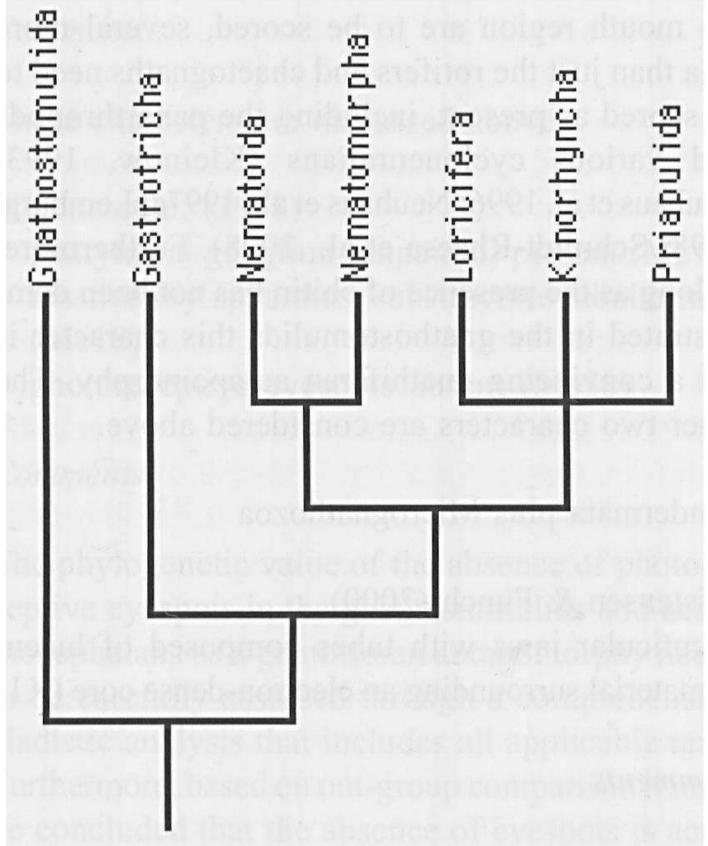

Fig. 7. Phylogenetic position of Gnathostomulida according to Wallace et al. (1996).

but this will not be attempted here. Additionally, it is noted that the observed scoring for Wall is not logically consistent. Gastrotricha is scored as polymorphic because striated muscles are known in this phylum, but this logic also demands a similar scoring of other taxa with polymorphic muscle cytology such as the Polychaeta and Rotifera.

A triangular or star-shaped pharynx lumen is not a compelling synapomorphy of the gnathostomulids and cycloneuralians. First, the pharynx of the gnathostomulids does not show a clearly triangular or starshaped lumen, and is therefore not particularly reminiscent of those of the cycloneuralians (Herlyn \& Ehlers, 1997). Second, the scoring of such a pharynx for the Priapulida (Wa23) is also not supported (Neuhaus, 1994; Nielsen, 2001). Moreover, triradiate pharynges are also found in various other taxa, including the pycnogonids, ectoprocts, onychophorans, and tardigrades (Ruppert, 1982; SchmidtRhaesa et al., 1998; Nielsen, 2001), taxa which were not included in the analysis of Wallace et al. (1996). However, Ruppert (1982) and Nielsen (2001) conclude that an overall homology of this pharynx shape is not likely. At least, the analysis of Wallace et al. (1996) has not included enough taxa (and misscored 
the Gnathostomulida and Priapulida) to properly evaluate the phylogenetic support of this character for placing the gnathostomulids.

Interestingly, when Wall is rescored for the Gastrotricha as simply possessing smooth muscles (no polymorphism, in agreement with the other scored terminals), and when Wa23 is rescored for the priapulids and gnathostomulids, the position of the gnathostomulids remains unresolved (strict consensus after exhaustive search).

\section{(Gnathostomulida Lobatocerebrum Nemertea 'Trochozoa')}

Proposed synapomorphies: Haszprunar (1996a) (based on tree B2 in his fig. 1.1).

Haszprunar (1996a)

- frontal gland present $\rightarrow$ absent (H17)

\section{Comments}

This character does not support the proposed sister grouping unequivocally because the first two taxa diverging after the Gnathostomulida (Lobatocerebrum and Nemertea) are scored as possessing frontal glands.

\section{Placing Gnathostomulida: comparison of alter- native hypotheses}

Which of the five discussed competing sister group hypotheses for Gnathostomulida is most firmly grounded in available morphological evidence? One proposed sister group, namely (Lobatocerebrum Nemertea Trochozoa Myzostomida) can be rejected outright. The sole character supposedly supporting this sister group hypothesis is equivocal and may thus be optimized on a different node in the cladogram. Moreover, restrictive taxon sampling did not allow Haszprunar's (1996a) analysis to test this hypothesis against any other alternative except the Plathelminthomorpha Hypothesis (but see below). The remaining hypotheses will be analyzed according to the set of criteria outlined at the beginning of this paper.
The two characters proposed to support a cycloneuralian affinity are either more widespread among metazoans and therefore potentially plesiomorphic on this level (smooth muscle absent), or invalid because the gnathostomulids lack this feature (triangular or star-shaped pharynx). Moreover, experimental rescoring of these features leaves the position of gnathostomulids unresolved.

Restrictive taxon sampling in the studies of $\mathrm{Ax}$ (1985, 1989, 1995), Ahlrichs (1995), Haszprunar (1996a, b), Kristensen \& Funch (2000), Melone et al. (1998), and Eernisse et al. (1992) compromises their testing power for the placement of the gnathostomulids. This is also reflected in the failure to fulfill the domain of definition for many of the characters in these studies. It must be concluded that these studies are a priori biased so that certain sister group relationships could in principle not be found. This is of course obvious for the manual cladistic analyses that do not allow a globally most parsimonious solution across all taxa. The consideration of restricted sets of taxa at any one time guarantees that the range of possible tree topologies is always heavily predetermined by taxon selection. However, even though the analysis of Wallace et al. (1996) included all taxa that have so far been proposed as sister groups to Gnathostomulida, taxon selection is restrictive because not all potential sister groups of the other taxa are included, e.g., nemerteans.

Differences in the proposed cladistic placements of the gnathostomulids may partly be reduced to differences in character sampling between the different cladistic analyses. For example, Sørensen et al. (2000) and Nielsen (2001) supported the Gnathifera hypothesis, but these studies excluded many of the characters that were suggested as being diagnostic apomorphies of a monophyletic Plathelminthomorpha in other studies. Conversely, Meglitsch \& Schram (1991) and Eernisse et al. (1992) supported the monophyly of Plathelminthomorpha, but neither of these analyses included a character on the similarities in jaw ultrastructure in the gnathostomulids and rotifers. It can therefore be concluded that the placement of the gnathostomulids in these latter two studies is no longer reliable. Naturally, this conclusion is only possible in retrospect, but it remains true that the results of these analyses are 
Table 4. Summary of alternative sister taxa for Gnathostomulida with diagnostic synapomorphies and comments. See text for discussion.

\begin{tabular}{ll}
\hline Sister taxon & Synapomorphies and source \\
\hline Platyhelminthes & see Table 2 \\
Gastrotricha & $\begin{array}{l}\text { loss of multiple protonephridial terminal cells (ZI30) } \\
\text { protonephridial filter formed by weir-like } \\
\text { fenestrations of the terminal cell's wall (ZI32) } \\
\text { serial protonephridia (ZI34) }\end{array}$
\end{tabular}

Comments

\section{Syndermata}

Cycloneuralia

Lobatocerebrum Nemertea Trochozoa (plus Myzostomida) z-elements of muscles present as isolated z-dots (A15) protonephridial canal cell surrounds lumen completely (A15; HaVIIIc)

buccal ganglion (integrated into pharynx?) (A15); pharyngeal ganglion (unpaired) (Mel1); HaVIIIa nervus pharyngeus (A15) pair of caudal ganglia (A15); caudal ganglion (HaVlllb)

cuticular hard parts connected across secreting epithelium by cross-striated muscles (A15); pharynx with cross-striated muscles, attached to jaw elements by epithelial cells (S43; NI35; ZII52)

esophagus present (A15)

gland characters (A15; HaVIIId)

jaw elements with tube-like support rods composed of electron lucent material surrounding an electrondense core (Ah1, HaVIIIa, Me18, K1, S42, NI34, G7) photoreceptive eyespots absent (Me58)

mouth region with chitinous membrane (NI63; ZII60) extended non-contractile regions of pharyngeal musculature (ZII59)

protonephridial filter formed by weir-like fenestrations of the terminal cell wall (ZII69)

protonephridial canal cell cilia absent (ZII70)

loss of sperm acrosome (ZII79)

smooth muscles absent (Wa11)

pharynx shape triangular or star-shaped (Wa23)

sperm lacking accessory centriole (Z1I83)

frontal gland present $\rightarrow$ absent (H17) convergent, and contentious scorings related to semaphoront choice

several misscorings

scored '?' for Gastrotricha, and problematic scoring for Annelida

domain of definition not fulfilled

ok

absent in Gnathostomulida

absent in Gnathostomulida

domain of definition not fulfilled

status as independent character is weak, and this mode of muscle attachment (irrespective of body region ) is more widespread than indicated by scorings

domain of definition not fulfilled

domain of definition not fulfilled ok

plesiomorphy in phylogeny of Melone et al. (1998) various misscorings

ok

misscored for many phyla

Ok

equivocal and Cycliophora misscored

possibly ok, but domain of definition not fulfilled misscorings for Gnathostomulida and Priapulida several misscorings

equivocal support and character coding and scoring is contentious currently superseded by the accumulation of new information. Similarly, the characters supporting the sister group relationship of the gnathostomulids and gastrotrichs in the analysis of Zrzavý et al. (2001) are uniquely included only in this study.

The potential effect of character coding is clearly illustrated by the experiment reported above for coding the gnathiferan jaw elements and the asso- ciated muscles as either one or two separate characters in the analyses of Peterson \& Eernisse (2001) and Nielsen (2001). Depending upon the nature of the data matrix under consideration, the decision to code this variation as one or two characters may have a rather dramatic impact or no noticeable impact at all (considering strict consensus trees) (Peterson \& Eernisse, 2001 and Nielsen, 2001, respectively). 
Character scoring problems are especially widespread among the proposed apomorphies for the Plathelminthomorpha (see under Platyhelminthes), but also for the sister groupings with Gastrotricha and Cycloneuralia. Scrutiny of the many synapomorphies proposed by Ahlrichs (1995) in support of a monophyletic Gnathifera revealed that two characters are no longer valid because these are lacking in the gnathostomulids (caudal ganglion and pharyngeal nerve). However, it should be clear that it is very difficult to assess the precise effects of the many scoring errors on the outcomes of the cladistic analyses. On the basis of the present discussion it is impossible to conclusively reject any of the proposed sister group hypotheses, except the one proposed by Haszprunar (1996a). The choice among the remaining alternatives requires the complete correction of all identified scoring errors, the removal of all taxon and/or character selection biases, and a reanalysis of the emended data set(s), which can only then function as a maximally effective cladistic test of available competing phylogenetic hypotheses. The current discussion is merely a first necessary step towards this ultimate goal. On the basis of character quality, a sister group relationship of the gnathostomulids and syndermates appears, for now, to be the best supported.

An interesting problem remains to be resolved. Despite the acknowledged weakness of the characters originally proposed in support of a monophyletic Plathelminthomorpha (widespread characters, probably convergent or plesiomorphic on this level) this clade is nevertheless supported in the majority of comprehensive cladistic studies. Intuitively, it could be expected that introduction of characters on the uniquely shared pharyngeal similarities with ultrastructurally identical jaw elements in the gnathostomulids and rotifers would immediately lead to the collapse of Plathelminthomorpha and a recovery of a clade Gnathifera.'This, obviously, did not happen. Despite the fact that the data sets of Wallace et al. (1996), Zrzavý et al. (1998), and Peterson \& Eernisse (2001) included this information, their morphological analyses preserved a monophyletic Plathelminthomorpha. Do these results therefore signify a strong and reliable phylogenetic signal for the Plathelminthomorpha?

As is discussed above, the character support for the Plathelminthomorpha in the analysis of Peterson
$\&$ Eernisse (2001) is very weak, and may be influenced by several coding and scoring difficulties. Also, rotifers, platyhelminths, and gnathostomulids together with Cycliophora form a clade. As an experiment, I left P21 (presence of perforatorium) out of the analysis because its status as a character independent from P20 is problematic. This resulted (heuristic search, 100 random addition replicates, TBR branch swapping) in the collapse of the whole bilaterian clade, exactly similar to the change in topology when a novel character on muscle-jaw attachment was introduced into the data set (see above). This result left the relationships between the platyhelminths, gnathostomulids and rotifers totally unresolved. It must be concluded that the instability of the results in the face of small changes in the data matrix of Peterson \& Eernisse (2001) indicates that there is no significant support for the Plathelminthomorpha Hypothesis. A rescoring experiment reported above for the data set of Wallace et al. (1996) leads to the same conclusion.

Although I have not performed any experimental reanalyses of the large matrix of Zrzavý et al. (1998), the secondary loss of an anus is the sole unique synapomorphy for the platyhelminths and gnathostomulids. The remaining characters exhibit a variety of problems that need to be resolved before the true character support for Plathelminthomorpha can be estimated.

Monophyly of the Gnathifera is not well supported in terms of sheer numbers of apomorphies. However, the quality of the unique similarities in pharyngeal morphology and the discovery of the Micrognathozoa that exhibits identical pharynx morphology to the rotifers and gnathostomulids lead to the conclusion that Gnathifera is the most robustly supported clade. Other features of Micrognathozoa such as the intracellular skeletal lamina similar to those in syndermates (but not a syncytial epidermis) further strengthens the gnathiferan nexus (Kristensen \& Funch, 2000; Sørensen et al., 2000).

\section{Placing Gnathostomulida in molecular and total evidence analyses}

On the basis of 18S rDNA sequence data Littlewood et al. (1998) found evidence for a sister group relationship between the gnathostomulids (one se- 
quence) and the chaetognaths forming a sister clade to a clade of nematodes. However, forcing a cladogram topology congruent with either the Plathelminthomorpha or Gnathifera Hypotheses, Littlewood et al. (1998) found trees six and two steps longer than the original 3081 steps. These three solutions were not statistically significant from one another. Unfortunately, the gnathostomulids, chaetognaths, and nematodes included in Littlewood et al's analysis were among the taxa with the longest branches, which could have caused them to group on the basis of chance similarities (long branch attraction). Indeed, a later study by Ruiz-Trillo et al. (1999) supported the conclusion that the gnathostomulid sequence and one of the chaetognath sequences used in the analysis of Littlewood et al. have significantly higher substitution rates then most other taxa.

In contrast, the molecular analysis of Zrzavý et al. (1998) suggested a sister group relationship of the gnathostomulids [the same sequence as in Littlewood et al., 1998] and the gastrotrichs positioned low within the Bilateria, but in the absence of any measures of support, or information on branch lengths, it is virtually impossible to assess the robustness of this topology.

Giribet et al. (2000) added two new gnathostomulid sequences to their analysis and found support for a clade of Gnathostomulida + Cycliophora (the latter was not included in the previous two studies). This clade was part of a larger protostomian clade, Platyzoa, that also included the syndermates, platyhelminths, and gastrotrichs.

The results of the analysis by Zrzavý et al. (2001) are comparable to those of Giribet et al. (2000). Gnathostomulida is placed as a sister group to Gastrotricha as part of a clade Platyzoa with the difference that this clade additionally includes the newly sequenced myzostomids.

Peterson \& Eernisse (2001) positioned the gnathostomulids at the base of the Bilateria as a sister group to acoels, but they conclude that the long branches of these taxa could cause them to be artificially attracted to the non-bilaterians which are separated from the bilaterians by a long branch.

The results of a relative rate test performed by Ruiz-Trillo et al. (1999) suggest that the gnathostomulid sequence used in Littlewood et al. (1998), Zrzavý et al. (1998, 2001), and Peterson \& Eernisse
(2001), and at least one of the three sequences used in Giribet et al. (2000), exhibit a relatively high substitution rate, which introduces the danger of long branch attraction. This possibly also explains the position of gnathostomulids basal in the Bilateria in the trees of Zrzavý et al. (1998) and Peterson \& Eernisse (2001) as due to an artifact of long branch attraction between the gnathostomulid sequence and the long branch separating the bilaterians from the non-bilaterians. Interestingly, Jondelius et al. (2002) provided the first molecular support for a sister group relationship of the gnathostomulids and syndermates as part of a basal bilaterian clade that diverges after the split of acoels and nemertodermatids and the other bilaterians. However, although these are the first molecular results fully congruent with morphological support for a monophyletic Gnathifera (molecular data for Micrognathozoa is currently lacking), they should be interpreted with caution. The single gnathostomulid sequence used in Jondelius et al. (2002) does not pass their relative rate test, and it is the same sequence that is rejected on the basis of the relative rate test in Ruiz-Trillo et al. (1999). Instead, Jondelius et al. (2002) tried to circumvent potential long branch attraction problems by reducing the amount of homoplasy in their data set by recoding their data as nucleotide triplets. Nevertheless, in their maximum parsimony analysis the gnathostomulids group with syndermates and a nematomorph at the base of the Bilateria (minus acoels and nemertodermatids). None of the sequences of these taxa passed the relative rate test, which led to their exclusion from the maximum likelihood analysis in Jondelius et al. (2002). Thus, on the basis of current molecular information, we cannot decide whether the gnathostomulids are platyzoan lophotrochozoans or basal bilaterians, i.e., part of a clade that diverges from the remaining Bilateria after acoels and nemertodermatids have split off.

The total evidence analysis of Zrzavý et al. (1998) supported a sister group relationship of Gnathostomulida and Gastrotricha that was also apparent in the molecular analysis, and this clade was maintained even when a weighting ratio of $7 / 1$ was applied to morphological/molecular data sets.

The total evidence analysis of Giribet et al. (2000), which included the same morphological data set as 
Zrzavý et al. (1998) with the exception that they excluded several taxa, supported Gnathostomulida as a sister taxon to a clade of Cycliophora + Syndermata.

The total evidence analysis of Zrzavý (2003) supported a close relationship between Gnathostomulida and the syndermates (including Micrognathozoa).

When the total evidence analyses of Zrzavý et al. (1998) and Giribet et al. (2000) (their figs. $3+$ 7 , and $4+5$, respectively) are compared a striking conclusion presents itself. Despite some topological congruence, the degree of incongruence is remarkable, which can be illustrated by considering the overall position of gnathostomulids in the trees. Zrzavý et al. (1998) place gnathostomulids at the base of their clade Ecdysozoa. In contrast, Giribet et al. (2000) place them far removed from ecdysozoans in a clade Platyzoa, which as a whole constitutes the sister group to a clade of the remaining non-ecdysozoan protostomes. Because the morphological data of these analyses were identical, this topological discrepancy must chiefly reside in different molecular phylogenetic signals. The two analyses differ mainly in two respects, one empirical, and one relating to methods of sequence comparison. First, Giribet et al. (2000) included 37 additional metazoan sequences (19 of these were molluscan and arthropodan), while excluding all non-bilaterians included in Zrzavý et al. (1998). Because the non-bilaterians are separated from the bilaterians by a long branch (see Giribet et al., 2000 and Peterson \& Eernisse, 2001), this may have an effect on the placement of the long branch gnathostomulid sequence. Second, and perhaps more importantly, Zrzavý et al. (1998) employed a traditional sequence alignment procedure, whereas Giribet et al. (2000) used direct optimization (see their paper for details). This probably explains most of the incongruence between these two total evidence studies, especially since both used the same analysis parameters, i.e., equal weighting of both molecules and morphology, and transversions and transitions.

The total evidence analysis of Zrzavý et al. (2001) unsurprisingly yielded a sister group relationship between Gnathostomulida and Gastrotricha, which is also supported by the molecular and morphological data sets separately.
The total evidence analysis of Peterson \& Eernisse (2001) supported gnathostomulids as the sister taxon to all Bilateria except acoelomorphs.

It is to be expected that the total evidence placement of gnathostomulids is equally adversely affected by the potential long branch attraction as the strictly molecular placements. The problematic molecular data combined with the difficulties in character scoring and selection in the morphological data sets currently prevent any unambiguous conclusion to be drawn concerning the phylogenetic placement of the gnathostomulids in total evidence analyses.

\section{Part II: Character evaluations}

The previous section discussed the character support for competing phylogenetic hypotheses for placing Platyhelminthes, Nemertea, and Gnathostomulida within the Metazoa. This part puts these character discussions into a broader context by extending them beyond the 'acoelomate' worms. This part will also provide the necessary justifications for several of the conclusions of Part I. The overall phylogenetic significance of the characters across the Metazoa is evaluated by focusing on how different cladistic analyses employ the same characters.

The character discussions follow a general layout. First, the character will be defined. Second, all observed character codings of the character across different studies will be listed. Third, character scoring conflicts between different phylogenetic studies will be tabulated and discussed. Note that the tables of observed scoring conflicts do not necessarily include all characters listed under Character coding because not all taxa are included in each study, some studies did not provide an explicit data matrix, and in some instances the codings differ too much for a straightforward comparison of their scorings. Fourth, the phylogenetic significance of the character will be evaluated. It should be noted that the following character discussions offer no definitive comparative treatments. The main aim of this section is to identify and shed some light on controversial character interpretations, and to identify the limits of our understanding of the phylogenetic significance of the characters. 
This section focuses primarily on character scoring issues. Special problems with character coding are in some cases discussed, but I have not attempted to do so comprehensively. For example, it may not be obvious in some instances how many character states should be used to encompass a particular spectrum of organismic variation. In addition, the treatment of inapplicable characters (necessary when different characters are coded for the absence/presence of a feature, and for the different forms of the feature when it is present) varies widely between studies. For a general discussion of these issues the reader may consult Jenner (2002). That paper specifically addresses pitfalls of currently adopted character coding strategies in metazoan cladistics for all characters included in five of the most recent cladistic analyses of the Bilateria or Metazoa incorporating morphological data (Giribet et al., 2000; Sørensen et al., 2000; Nielsen, 2001; Peterson \& Eernisse, 2001; Zrzavý et al., 2001). The analyses of Zrzavý (2003) and Brusca \& Brusca (2003) were not available for that study.

The following is a list of all discussed characters. Those marked with an asterisk have been discussed above in connection with the relevant studies, whereas the unmarked characters are discussed in this section.

\section{Epidermis and cuticle}

Multiciliate epidermal cells

Cuticle layers

\section{Reproduction and sexual condition}

\section{Sexes}

Asexual/sexual reproduction

Mode of sperm deposition and mode of fertilization Filiform sperm

Spermatozoa without accessory centriole

Spermatozoa with compact acrosome* (see under Alternative phylogenetic hypotheses for Platyhelminthes)

Spermatozoa with perforatorium

Sperm flagella

Gonads asacular or sacular

Gonocoel

\section{Development}

Spiral cleavage

Blastula

Fixed cell fate during cleavage* (see under Alternative phylogenetic hypotheses for Platyhelminthes)

Mesodermal germ bands derived from $4 d^{*}$ (see under Alternative phylogenetic hypotheses for Nemertea)

\section{Larval characters}

Prototroch

Metatroch

Larvae or adults with downstream-collecting ciliary system

Larva with strongly reduced hyposphere

Prototrochal lobes

General shape of ciliary bands in platyhelminths and nemerteans* (see under Alternative phylogenetic hypotheses for Platyhelminthes)

Ciliated larvae without bivalved shells* (see under Alternative phylogenetic hypotheses for Nemertea)

Pelagic larvae with apical ciliary tuft and plate* (see under Alternative phylogenetic hypotheses for Nemertea)

Cerebral rhabdomeric larval ocelli or integumentary pigment cups* (see under Alternative phylogenetic hypotheses for Nemertea)

\section{Body cavities}

Secondary body cavity, coelom

Histological (ultrastructural), morphological, structural definition

Ontogenetic source

Developmental or morphogenetic mode

Coelomocytes

Lateral coelom derived from mesodermal bands* (see under Alternative phylogenetic hypotheses for Nemertea)

\section{Nervous system and sensory organs}

Statocysts

Adults with apical nervous system only* (see un- 
der Alternative phylogenetic hypotheses for Platyhelminthes)

Cerebral ganglion, brain

Adult brain derived from or associated with larval apical organ

Serial repetition of nerve collaterals

Orthogonal nervous system (see also under Alternative phylogenetic hypotheses for Platyhelminthes and below under Serial repetition of nerve collaterals)

Two ventrolateral or one primitively paired ventral nerve cord* (see under Alternative phylogenetic hypotheses for Nemertea)

Ventral nervous system* (see under Alternative phylogenetic hypotheses for Platyhelminthes and Alternative phylogenetic hypotheses for Nemertea)

Buccal (pharyngeal) ganglion* (see under Alternative phylogenetic hypotheses for Gnathostomulida)

Pharyngeal nerves* (see under Alternative phylogenetic hypotheses for Gnathostomulida)

Caudal ganglion* (see under Alternative phylogenetic hypotheses for Gnathostomulida)

\section{Nephridial characters}

Protonephridia

Podocytes/terminal cells/nephrocytes

Multiple protonephridial terminal cells* (see under Alternative phylogenetic

hypotheses for Gnathostomulida)

Protonephridial terminal cell fenestrations* (see under Alternative phylogenetic hypotheses for Gnathostomulida)

Serial protonephridia* (see under Alternative phylogenetic hypotheses for Gnathostomulida)

Protonephridia with channel cell completely surrounding lumen

\section{Muscles}

Muscle cells

Subepidermal muscle sheet derived from $4 d-$ mesentoblast* (see under Alternative phylogenetic hypotheses for Platyhelminthes)

Dermal circular (or external transverse) muscular fibers* (see under Alternative phylogenetic hypotheses for Nemertea)

Z-elements present as Z-dots* (see under Alternative phylogenetic hypotheses for Gnathostomulida)

Cross-striated pharynx muscles connected to jaw elements through epithelial cells* (see under Alternative phylogenetic hypotheses for Gnathostomulida)

Smooth muscles* (see under Alternative phylogenetic hypotheses for Gnathostomulida)

\section{Digestive system}

Intestinal cell ciliation

Anus

Complete gut with mouth arising from blastopore* (see under Alternative phylogenetic hypotheses for Nemertea)

Jaw elements with tube-like support rods composed of electron lucent material surrounding an electron-dense core* (see under Alternative phylogenetic hypotheses for Gnathostomulida)

Mouth region with chitinous membrane* (see under Alternative phylogenetic hypotheses for Gnathostomulida)

Pharynx shape triangular or star-shaped* (see under Alternative phylogenetic hypotheses for Gnathostomulida)

\section{$\underline{\text { Miscellaneous characters }}$}

Frontal gland complex

Locomotion

Lack of mitosis in somatic or epidermal cells (eutely)

Chitin and chitinase* (see under Alternative phylogenetic hypotheses for Platyhelminthes)

Lophotrochozoan complement of Hox genes

Septate junctions

Closed blood vessels

Segmented or serial mesodermal structures* (see under Alternative phylogenetic hypotheses for Nemertea)

Compound cilia

Respiratory pigments

Gliointerstitial cell system 


\section{Epidermis and cuticle}

\section{Multiciliate epidermal cells}

Multiciliate epidermal cells may have multiple separate cilia or compound cilia.

\section{Character coding}

S12: ciliation of epidermal cells monociliate/multiciliate/no cilia

P13: ciliated epidermis a/p with monociliated cells/ $\mathrm{p}$ with multi- or mono- and multiciliated cells P14: densely multiciliated epidermis $a / p$

H4; Wa7; ZII25: epidermis with monociliated cells/ polyciliated cells

E31: multiciliary epidermis with ciliated rootlets $\mathrm{a} / \mathrm{p}$

Table 5. Scoring conflicts of multiciliated epidermal cells.

\begin{tabular}{|c|c|c|c|c|}
\hline & Absent & $\begin{array}{l}\text { Present (also } \\
\text { polymorphic) }\end{array}$ & $?$ & $\begin{array}{l}\text { Proposed } \\
\text { scoring }\end{array}$ \\
\hline Phoronida & $\begin{array}{l}\text { P13; E31; } \\
\text { ZII25 }\end{array}$ & $\mathrm{S} 12$ & & Absent \\
\hline Brachiopoda & $\begin{array}{l}\text { P13; E31; } \\
\text { Z1125 }\end{array}$ & $\mathrm{S} 12$ & : & Absent \\
\hline Chordata & E31 & P13 & ZII25 & Absent \\
\hline
\end{tabular}

\section{Character scoring and phylogenetic significance}

Epidermal cells in the phoronids are typically monociliate (Herrmann, 1997). The presence of multiciliated epidermal cells as is scored for S12 is doubtful. Although Gilmour (1978) reported the presence of multiciliated cells on the tentacles of actinotroch larvae, subsequent studies have not confirmed their presence (Nielsen, 1987; Pardos et al., 1991; Nielsen \& Riisgård, 1998). Only monoand sparse biciliate cells were reported.

A similar situation pertains to the reports of multiciliated epidermal cells in Brachiopoda. Whereas Gilmour (1981) reported the presence of multiciliated tentacular epidermal cells, their presence was not confirmed by later studies (Nielsen, 1987; Nielsen \& Riisgård, 1998; Nielsen, 2001).

The presence of multiciliate epidermal cells in the ground pattern of Chordata (P13) is unlikely given the predominance of non-ciliated epidermal cells in chordates and the presence of scattered monociliated cells in the epidermis of cephalochordates (Ruppert, 1997; Nielsen, 2001).

The distribution of multiciliate epidermal cells recorded in the analyses of Sørensen et al. (2000) and Peterson \& Eernisse (2001) is consistent with their interpretation as a autapomorphy of Acrosomata (equivocal in Sørensen et al., 2000). However, convergence of multiciliate cells is likely, for example, as is indicated by their sporadic occurrence in the cnidarians (Nielsen, 2001) and the likelihood of their evolution within Gastrotricha (Hochberg \& Litvaitis, 2000). In a recent study Zrzavý (2003: 73 ) concluded in contrast to traditional ideas (see Hochberg \& Litvaitis, 2000) that "ancestral gastrotrichs were originally multiciliate regardless of their final phylogenetic position," and that "primitive monociliation [traditional view] is less parsimonious." However, this conclusion cannot be upheld.

The hypothesis of relationships within the gastrotrichs proposed by Zrzavý (2003: fig. 5) suggests that the monociliate Dactylopodolida are the sister group to the remaining, chiefly multiciliate, gastrotrichs. This leaves the gastrotrich ground pattern of epidermal cell ciliation uncertain. Consequently, the primitive form of ciliation is dependent upon the state found in out-groups, in contrast to Zrzavý's claim that the ancestral pattern of ciliation for the gastrotrichs can be determined "regardless of their final phylogenetic position" (Zrzavý, 2003: 73).

The analysis of Zrzavý (2003) suggests that the gastrotrichs are the sister phylum to the Ecdysozoa. Zrzavý (2003: 73) concluded that "Ecdysozoa were probably multiciliate originally." However, because epidermal cilia are generally lacking in the ecdysozoans, these phyla were all scored as "?s", with the exception of the onychophorans, which were scored as possessing multiciliate cells. The presence of multiciliate cells in the metanephridia of the onychophorans is the basis of this scoring in Zrzavý (2003: 73). However, because this character (ZII25) supposedly codes for the ciliation of epidermal cells, the onychophorans should be rescored. Bartolomaeus \& Ruhberg (1999) identified monociliate epidermal cells in the developing embryo. A recent 
study by Eriksson et al. (2003: fig. 51) confirmed the presence of monociliate cells in the hypocerebral organ of the onychophorans. This organ develops from invaginated ectodermal cells. When the onychophorans are rescored accordingly, the ground pattern of the gastrotrichs remains uncertain when the character is mapped on Zrzavý's morphological phylogeny. When epidermal cell ciliation is optimized on Zrzavý's (2003) preferred total evidence phylogeny, monociliate cells are inferred to be the primitive condition for the ecdysozoans, with a reversal to multiciliate cells within the gastrotrichs.

\section{$\underline{\text { Cuticle layers }}$}

Cuticles that cover the body surface of the metazoans come in many guises, from a simple, thin glycocalyx interspersed between epidermal microvilli to thick, multilayered 'true' cuticles that may even form adorning structures such as spines, scales and hooks, and that may overlay epidermal cells lacking microvilli (Rieger, 1984). Different types of cuticle are generally distinguished on the basis of differences in the number of layers, their ultrastructural appearance, e.g., granular, fibrillar, laminate, and their chemical composition (containing collagen or chitin). A general terminology for cuticle layers applicable across different phyla distinguishes an outermost epicuticle (often laminate), a middle exocuticle (often proteinaceous), and a innermost endocuticle (often fibrillar) (see Lemburg, 1998 and Schmidt-Rhaesa et al., 1998 for useful reviews).

\section{Character coding}

$\mathrm{H1}$; Z193: cuticle simple/two-layered

ZI21: molted cuticle with epicuticle, exocuticle, and endocuticle, with sclerotization $\mathrm{a} / \mathrm{p}$

ZII35: cuticle with epicuticle and basal layer $\mathrm{a} / \mathrm{p}$ ZII36: molted sclerotized cuticle with the basal layer differentiated as exocuticle and endocuticle $a / p$ Eh3: stratified cuticle with epicuticle and basal layer $\mathrm{a} / \mathrm{p}$

\section{Character scoring and phylogenetic significance}

Although no attempt is made here to present a complete discussion of cuticular organization throughout the Metazoa, some comments on the adopted coding and scoring of $\mathrm{H} 1$ and $\mathrm{Z} 193$ are in order. Haszprunar (1996a) introduced a character (H1) involving layering of the cuticle into a cladistic analysis of the spiralians. Haszprunar (1996a) based his character coding primarily upon earlier work by Rieger $(1980,1981,1984)$. The two character states of $\mathrm{H} 1$ distinguish a simple cuticle, which is equivalent to a glycocalyx, from a more complex cuticle, which is composed of an outer thin epicuticle and a thicker basal layer. The latter character state was proposed as a synapomorphy of the Trochozoa, Nemertea and Lobatocerebrum (with one reversal to a glycocalyx covered epidermis in Nemertea). However, the phylogenetic significance of a layered cuticle could not be fully appreciated within the context of the limited sample of protostome phyla included in Haszprunar's study.

Subsequently, Zrzavý et al. (1998) introduced the same character (Z193) with identical coding for the first time into a cladistic analysis of all metazoan phyla. However, the scoring of Z193 does not accurately reflect the cuticular organization of many phyla. Although all phyla also included in the analysis of Haszprunar (1996a) were scored identically (and correctly) for Z193, the scoring for many other bilaterian phyla presents problems. First, all the lophophorate and deuterostomian phyla were scored as having a simple cuticle (glycocalyx only). This scoring ignores the (bi- or multi-) layered cuticles that are present in a number of these taxa, including the echinoderms, brachiopods, ectoprocts, possibly urochordates (often containing cells; fig. 8A in Burighel \& Cloney, 1997), and even the cnidarians (Rieger, 1980, 1984; Byrne, 1994; Cavey \& Märkel, 1994; Chia \& Koss, 1994; Heinzeller \& Welsch, 1994; Smiley, 1994; Mukai et al., 1997; Williams, 1997). The cuticles of these phyla exhibit varying degrees of similarity to those found in non-ecdysozoan protostomes, and are certainly not simple glycocalices.

Second, the bilaterian phyla that were scored as '?' for cuticle organization such as the chaetognaths, echiurans, and cycliophorans, also exhibit a 
cuticle ultrastructure that closely resembles the cuticle found in taxa that were scored as possessing a layered cuticle (Pilger, 1993; Funch, 1996; Funch \& Kristensen, 1997; Shinn, 1997).

Third, the panarthropods and cycloneuralians were scored as possessing two-layered cuticles, similar to those observed in the trochozoans and Lobatocerebrum. Interestingly, recent studies have yielded more detailed information on cuticle structure in these taxa that clearly shows their distinct organization, and which has been used to help resolve their relationships (Neuhaus, 1994; Neuhaus et al., 1996; Lemburg, 1995, 1998; Schmidt-Rhaesa et al., 1998, 2002). In particular, a bilayered cuticle with (tri)laminate epicuticle and proteinaceous basal layer is considered as a synapomorphy of the cycloneuralians (see also Eh3). A trilayered cuticle with a (tri)laminate epicuticle, a proteinaceous median layer (exocuticle) and a fibrillar basal layer (endocuticle) with chitin is considered a synapomorphy of either the introvertans or the ecdysozoans (Ehlers et al., 1996; Neuhaus et al., 1996, 1997b; Lemburg, 1998; Schmidt-Rhaesa et al., 1998; see Jenner, 2002 for a coding experiment of cuticles).

It should be noted that a considerable degree of ultrastructural variation may be observed between different phyla, as well as within single phyla according to the body region or the ontogenetic stage that is being considered. For example, Nematoida lack chitin in the basalmost cuticle layer, adult nematomorphans lack the trilaminate epicuticle, and the cycloneuralian pharyngeal and epidermal cuticle may show variation in both organization and composition (for example in spines and scalids), e.g., Ruppert (1982, 1991b), Neuhaus et al. (1997a), and Lemburg (1998). Wägele et al. (1999) and Wägele \& Misof (2001) recently criticized the phylogenetic value of a complex cuticle character, such as a trilayered cuticle with trilaminate epicuticle and chitinous endocuticle, and proposed to decompose it into smaller traits that show less than perfect congruence in their distribution. I agree with Zrzavý (2001) that such reductionism is of limited value when pushed too far, but it raises the interesting issue of how to demarcate characters. If it can be ascertained that different components of a complex character have independent evolutionary histories, then it is worthwhile to explore the phy- logenetic significance of the different parts of a complex character (see Jenner, 2002 for a character coding experiment of cuticle layers).

The exact pattern of cuticle evolution remains to be determined. Nevertheless, Rieger's (1984) suggestion of the likelihood of convergent specializations in cuticle structure in different lines of metazoans appears to be supported to a certain degree by recent ultrastructural studies. For example, an epicuticular organization similar to the trilaminate epicuticle proposed for the introvertan or ecdysozoan ground patterns has also been observed for the Cycliophora, some molluscs, and brachiopods. However, in the molluscs and brachiopods the mode of formation of the epicuticle appears to differ from that of the introvertans and panarthropods. In these latter groups the epicuticle is secreted by the tips of epidermal microvilli (Schmidt-Rhaesa et al., 1998). Finally, considerable variation in cuticular layering within monophyletic phyla further indicates the existence of at least a certain amount of convergent evolution (Ruppert, 1982; Wright, 1991).

\section{Reproduction and sexual condition}

\section{$\underline{\text { Sexes }}$}

A number of cladistic analyses have coded characters with alternative character states for the presence of separate sexes (gonochorism, dioecious sexual system), or hermaphroditism (monoecious sexual system). These studies did not attempt to distinguish different types of hermaphroditism, such as simultaneous or sequential hermaphrodites (protandry, protogyny), or whether the male and female gonads are separate or part of the same (hermaphroditic) organ.

\section{Character coding}

Z126; E117: sex condition gonochoristic/hermaphroditic

Ca6: sexes not always separate/always separate C22: animals dioecious/monoecious/dioecious (dioecious system in vertebrates is coded separately) Me40: reproduction monoecious/dioecious Wa28: hermaphroditism a/p 
Table 6. Scoring conflicts of sexual system (gonochoristic [dioecious]/hermaphroditic [monoecious]).

\begin{tabular}{|c|c|c|c|c|c|}
\hline & Gonochoristić & Hermaphroditic & $?$ & Polymorphic & Proposed scoring \\
\hline Urochordata & E117 (Chordata) & $\mathrm{C} 22$ & $\mathrm{Z} 126$ & & Hermaphroditic \\
\hline Pterobranchia & $\mathrm{Z126}$; $\mathrm{C} 22$ & & & $\mathrm{Ca} 6$ & Polymorphic \\
\hline Phoronida & $\mathrm{E} 117 ; \mathrm{C} 22$ & & $\mathrm{Z} 126$ & $\mathrm{Ca} 6$ & Polymorphic \\
\hline Ectoprocta & $\mathrm{Z} 126 ; \mathrm{C} 22$ & $\mathrm{Ca} 6$ & & & Hermaphroditic or polymorphic \\
\hline Kinorhyncha & E117 & Z126; Wa28 & & & Gonochoristic \\
\hline Priapulida & E117 & $\mathrm{Z} 126, \mathrm{Wa} 28$ & & & Gonochoristic \\
\hline Nematoda & $\mathrm{Z} 126 ; \mathrm{E} 117$ & $\mathrm{Wa} 28$ & & & Gonochoristic \\
\hline Nematomorpha & Wa28 & & $\mathrm{Z} 126$ & & Gonochoristic \\
\hline Polychaeta & Z126 & Wa28 & & & Gonochoristic \\
\hline Sipuncula & Z126 & & & $\mathrm{Ca} 6$ & Gonochoristic \\
\hline Mollusca & $\begin{array}{l}\text { E117 (based on } \\
\text { aculiferans) }\end{array}$ & & $\mathrm{Z} 126$ & & Gonochoristic \\
\hline Nemertea & & Z126 & & E117 & Gonochoristic \\
\hline Cnidaria & E117 & & $\mathrm{Z} 126$ & & $?$ \\
\hline
\end{tabular}

\section{Character scoring and phylogenetic significance}

The scoring conflicts reveal that there is substantial disagreement about the nature of the plesiomorphic sexual condition in various phyla.

It is beyond doubt that hermaphroditism is plesiomorphic for the urochordates as it is characteristic of almost all ascidians, all appendicularians, except the 'model system' Oikopleura dioica which has separate sexes, and all thaliaceans (Cloney, 1990; Godeaux, 1990; Galt \& Fenaux, 1990; Burighel \& Cloney, 1997; Fenaux, 1998). Consequently, the scoring of Z126 must be changed. The scoring of gonochorism for Chordata in Eernisse et al. (1992) is ambiguous because the urochordates are typically hermaphroditic whereas the cephalochordates and vertebrates are typically gonochoristic.

The unambiguous scoring of gonochorism for pterobranch hemichordates for Z126 and C22 is wrong. Although the rhabdopleurids are gonochoristic, the cephalodiscids can also be hermaphroditic (Goldschmid, 1996b; Benito \&` Pardos, 1997) This justifies the scoring of a polymorphism as is done for Ca6 (Christoffersen \& Araújo-de-Almeida, 1994, included the cephalodiscids and rhabdopleurids as separate terminal taxa).

The unambiguous scoring of gonochorism for the phoronids (Z126, C22) should be adjusted because both gonochoristic and hermaphroditic species are known (Zimmer, 1991; Herrmann, 1997), and the plesiomorphic state appears uncertain at present.
The scoring of gonochorism for the ectoprocts (Z126, C22) should be adjusted. While all known ectoproct colonies are hermaphroditic, common gonochoristic zooids are mostly restricted to the stenolaemates, while zooids are typically hermaphroditic in gymnolaemates and phylactolaemates. Although the phylogenetic relationships of the major groups of ectoprocts remains in dispute (Woollacott \& Harrison, 1997; Nielsen, 2001), a recent morphological cladistic analysis indicates that the stenolaemates are not likely to be representatives of the plesiomorphic ectoproct (Todd, 2000). Moreover, it should be noted that the terminal taxon Bryozoa (Ectoprocta) in Zrzavý et al. (1998) is solely comprised of gymnolaemates and phylactolaemates, and should thus be rescored.

The scoring of Z126 and Wa28 for the kinorhynchs is wrong. All known kinorhynchs have separate sexes (Needham, 1989; Kristensen \& Higgins, 1991; Lorenzen, 1996g).

Priapulids are plesiomorphically gonochoristic (although occasional hermaphroditic individuals are known), in contrast to the scoring for Z126 and Wa28 (Storch, 1991; Lemburg \& Schmidt-Rhaesa, 1999; Storch et al., 2000).

Nematoda typically have separate sexes (Bird \& Sommerville, 1989; Wright, 1991; Lorenzen, 1994), with occasional hermaphrodites, in contrast to the scoring for Wa28.

Nematomorphs are also gonochoristic (Bird \& Sommerville, 1989; Bresciani, 1991; SchmidtRhaesa, 1999) in contrast to the scoring for Z126. 
The unambiguous scoring of hermaphroditism for the polychaetes in Wallace et al. (1996) is misleading. Polychaetes are typically gonochoristic, but hermaphroditism has evolved multiple times independently within different polychaete families (Schroeder \& Hermans, 1975; Westheide, 1996; Fischer, 1999).

The polymorphic scoring of $\mathrm{Ca} 6$ for the sipunculans is overly cautious. Sipunculans are gonochoristic with the exception of one species (Golfingia minuta) (Rice, 1989, 1993).

Although Z126 records a '?' for the sexual system of Mollusca, it is very likely that possession of separate sexes is the plesiomorphic state for the phylum. With the exception of hermaphroditic solenogasters, for which hermaphroditism may be an autapomorphy within the Mollusca, all basal molluscan taxa such as the caudofoveates, polyplacophorans, and monoplacophorans typically have separate sexes. Gonochorism is also the primitive state for the gastropods, bivalves, cephalopods and scaphopods (Salvini-Plawen, 1990; Haszprunar, 1992; Eernisse \& Reynolds, 1994; Götting, 1996; Salvini-Plawen \& Steiner, 1996; Haszprunar \& Schaefer, 1997; Shimek \& Steiner, 1997; Ponder \& Lindberg, 1997).

Although hermaphroditic nemerteans are known, these typically are derived freshwater and terrestrial forms, and are mostly restricted to the monostiliferan hoplonemerteans, which are not representative of the nemertean ground pattern (Turbeville, 1991, 1996; Norenburg \& Stricker, 2002; Thollesson \& Norenburg, 2003). Separate sexes are more common in the phylum, and representative of the more basal taxa. Therefore gonochorism can be regarded as the plesiomorphic sexual condition.

Both gonochorism and hermaphroditism occur within Cnidaria, and the plesiomorphic state is uncertain (Schäfer, 1996).

Placozoa is scored '?' with regard to the sexual condition, but both sperm and oocytes have been observed within a single individual (Grell \& Ruthmann, 1991; Ruthmann, 1996). This may indicate that the animals are hermaphroditic. In contrast, this may indicate that the placozoans have internal fertilization. Lobatocerebrum is a hermaphrodite (Rieger, 1980), and orthonectid mesozoans are gonochoristic with only one exception (Ax, 1995; Haszprunar, 1996c).
The prevalence of scoring problems necessitates a re-analysis of the phylogenetic significance of the nature of the sexual system in the Metazoa. However, what is clear is that this character is quite variable, both between and within phyla, and for several phyla the ground pattern remains uncertain, e.g., cnidarians, and phoronids. Convergent changes in the sexual system are documented within many established monophyla such as the brachiopods (Long \& Stricker, 1991), nemerteans (Norenburg \& Stricker, 2002), molluscs (Eernisse \& Reynolds, 1994), annelids (Schroeder \& Hermans, 1975; Glasby et al., 2000), tardigrades (Dewel \& Dewel, 1997), and nematodes (Lorenzen, 1994).

An interesting question that remains to be addressed is what polarity of evolutionary change is more common: from gonochorism to hermaphroditism, or vice versa? In order to arrive at a definitive answer a robust phylogenetic framework is needed, but as a first estimate on the basis of a review of the published literature I think that there is a bias towards the evolution of hermaphroditism from gonochorism. Even in highly speciose taxa that are plesiomorphically hermaphroditic such as the clitellates, platyhelminths or urochordates, changes from hermaphroditism to separate sexes appear to be very rare. This picture is consistent with the situation found in less speciose, and primitively hermaphroditic, phyla such as the gnathostomulids, ctenophores, and chaetognaths, where changes to separate sexes are unknown. Interestingly, in the one primitively hermaphroditic phylum where a significant number of species did לhange their sexual system, the Gastrotricha, the change has not been towards gonochorism but rather to a unique life cycle of parthenogenetic reproduction followed by simultaneous hermaphroditism during the life of a single individual (chaetonotid gastrotrichs: Weiss, 2001). In contrast, many instances of the evolution of hermaphroditism are know in primitively gonochoristic phyla (see discussion above and Ghiselin, 1969). Obviously, this problem needs to be approached on a much finer scale as well, because gonochoristic and hermophroditic individuals may even occur within the same species. 


\section{Asexual/sexual reproduction}

Asexual (vegetative) reproduction may be effected through a diversity of means such as budding, fission, or development from an unfertilized ovum (parthenogenesis). Asexual reproduction is the sole means by which many phyla form their distinctive colonies. However, it seems hardly possible to find sufficient similarities between the diverse modes of asexual reproduction in different phyla to propose primary homology of all these processes throughout the entire Metazoa.

\section{Character coding}

$\mathrm{H} 30$; Z128: asexual (vegetative) reproduction dominant/rare, absent

Wa33: agamic reproduction (fission or budding) $\mathrm{a} / \mathrm{p}$

Wa27: parthenogenesis a/p

\section{Character scoring and phylogenetic significance}

First, it should be noted that the alternative character states for $\mathrm{H} 30$ and $\mathrm{Z} 128$ are not unambiguously determined as long as no explicit cut-off point is defined that separates rare from dominant occurrence of asexual reproduction. The relative frequency of a trait within a higher taxon is less relevant for reconstructing a ground pattern than is the distribution of that trait within that group. The scored ground patterns are thus inherently uncertain for $\mathrm{H} 30$ and $\mathrm{Z} 128$.

Asexual reproduction by parthenogenesis and budding (paratomy) is the dominant reproductive mode in the catenulid platyhelminths (Rieger, 1986b; Rieger et al., 1991b), contra the scoring of H30.

Asexual reproduction is unknown in the gnathostomulids, justifying the scoring of $\mathrm{H} 30$.

Asexual reproduction by diverse means is found in various polychaetes and clitellates (Schroeder \& Hermans, 1975; Needham, 1990), but whether the ability for asexual reproduction is primitive for these taxa appears uncertain.

Dominant asexual reproduction is an imprecisely defined character that is certainly not a reliable autapomorphy for Plathelminthomorpha (scored "?". for several platyhelminths and gnathostomulids).
Table 7. Scoring conflicts for relative frequency of asexual reproduction.

\begin{tabular}{lllll}
\hline & $\begin{array}{c}\text { Dominant Rare or } \\
\text { absent }\end{array}$ & $\begin{array}{l}\text { Proposed } \\
\text { scoring }\end{array}$ \\
\hline Catenulida & $\mathrm{Z} 128$ & $\mathrm{H} 30$ & & Dominant \\
Gnathostomulida & & $\mathrm{H} 30$ & $\mathrm{Z} 128$ & Absent \\
$\begin{array}{l}\text { Polychaeta } \\
\text { Clitellata }\end{array}$ & $\mathrm{H} 30$ & $\mathrm{Z} 128$ & $?$ \\
\hline
\end{tabular}

The scoring of this character in Zrzavý et al. (1998) is puzzling. For example, dominant asexual reproduction is scored for taxa where: 1) the normal life cycle consists of an asexual and sexual part such as in Cycliophora (Funch \& Kristensen, 1997), 2) where asexual species are known but where asexual reproduction is neither the only reproductive mode, nor present in the majority of species, such as in Echinodermata (Holland, 1991; Chia \& Walker, 1991; Hendler, 1991; Smiley et al., 1991), 3) where the scoring is simply incorrect, such as in Nemertodermatida (Lundin \& Sterrer, 2001), Enteropneusta (Hadfield, 1975), Cephalochordata (Ruppert, 1997; Whittaker, 1997), scalidophorans and nematoidans (Lorenzen, 1996b). In contrast, the urochordates, which frequently exhibit asexual reproduction, are unambiguously scored as showing rare or no asexual reproduction. The unambiguous scoring of rare or absent asexual reproduction in the entirely parthenogenetic bdelloid rotifers seems to indicate that only asexual reproduction through budding or fission processes are scored for Z128. Furthermore, a certain extent of convergent evolution of asexual reproduction appears undeniable in taxa where sexual reproduction is the dominant and arguably primitive mode, such as in Sipuncula (Rice, 1975), Pogonophora (according to Southward, 1975 asexual reproduction is only present in Sclerolinum species; see Halanych et al., 2001 and Rouse, 2001 for molecular and morphological estimates of its phylogenetic position within the pogonophores), and Tardigrada (Pollock, 1975; Dewel et al., 1993). It is clear that a thorough re-assessment of asexual reproduction for future cladistic analyses is imperative.

.Wa33 scored agamic reproduction present for priapulids, but all known priapulids reproduce solely sexually (Lemburg \& Schmidt-Rhaesa, 1999; Storch et al., 2000). 
Wa27 codes for the presence of parthenogenesis, and the gastrotrichs, nematodes, and rotifers are unambiguously scored as possessing parthenogenesis. Although the presence of parthenogenetic reproduction in bdelloid (obligate) and monogonont (facultative) rotifers provides some certainty about the ground pattern state (not conclusive since Seison reproduces sexually), it is far from obvious that parthenogenesis is part of the nematode ground pattern (Lorenzen, 1996a) The phylogenetic distribution of parthenogenesis in gastrotrichs clearly suggests that it evolved within that phylum (Hochberg \& Litvaitis, 2000; Weiss, 2001). These findings support convergent evolution of parthenogenesis in these phyla.

The evolutionary significance of asexual modes of reproduction has fascinated zoologists throughout the history of zoology. Interestingly, from Huxley $(1859)$ to Buss $(1983,1987)$ research on asexual reproduction can be regarded as taking place within the same broader context: to arrive at a satisfactory explanation of the nature and origin of individuality. In a first attempt at classifying the divergent modes of asexual reproduction found throughout the animal kingdom, Huxley (1859:219) wrote that "all these modes of development pass into one another." [See Desmond, 1994 for the fascinating context of Thomas Huxley's work on parthenogenesis, and the role of this research in fueling his own ascent in the scientific community by polarizing his views opposite to those of the zoological doyen of Victorian England, Richard Owen.] However, in view of the lack of similarity between the different modes of asexual reproduction, one cannot maintain the validity of a broadly construed character such as H30 and Z128. Nevertheless, various recent hypotheses for the origin of higher level taxa have assumed the importance of asexual processes of reproduction, especially when budding results in colonial organization. The Eumetazoa, Bilateria, Deuterostomia, and even Vertebrata have recently been derived from a primitively colonial organization (Rieger, 1986a, 1988, 1994a, b; Rieger et al., 1991a; Lacalli, 1997, 1999, 2000; Dewel, 2000 ), and coloniality in phyla such as the entoprocts, pterobranchs and urochordates may consequently be regarded as plesiomorphic. However, the hopes for phylogenetically linking coloniality in different phyla should be tempered by recent insights (largely from molecular phylogenetics) that suggest that coloniality, even where it occurs in a dominant proportion of the species, has evolved independently within phyla. For example, although a cladistic framework of relationships is still lacking for entoprocts, there appears to be agreement among recent workers that the solitary entoprocts of the family Loxosomatidae may represent a paraphyletic grade giving rise to the monophyletic colonial forms (Nielsen, 1995; Emschermann, 1996). $18 \mathrm{~S}$ rDNA sequence data suggest that the colonial pterobranchs arose from within the solitary enteropneusts (Cameron et al., 2000; however, large subunit rDNA places the pterobranch Cephalodiscus as a sister group to enteropneusts: Winchell et al., 2002). Coloniality has arisen a number of times convergently within the ascidians (Swalla et al., 2000; Swalla, 2001; Stach \& Turbeville, 2002). One potential pathway to further our understanding of the evolution of asexual modes of reproduction, especially cases of budding or fission, that has proven profitable is to explore the relationship with regenerative capacities in both adult and larval metazoans, especially in terms of the deployment of similar sets of genes in these forms of non-embryonic development (Vickery \& McClintock, 1998; Bely \& Wray, 1999; Lacalli, 1999).

\section{Mode of sperm deposition and mode of fertiliza- tion}

Various studies have included characters coding for mode of sperm deposition or sperm transfer, and mode of fertilization. Clearly, these aspects of reproductive biology are as closely linked to each other, as they are to other characters such as sperm morphology (see under Filiform sperm). However, because an exact correlation is lacking, serious difficulties of character coding are introduced when broad ranges of phyla are analyzed simultaneously.

\section{Character coding}

A13: internal sperm deposition and internal fertilization

Direct sperm transfer and internal fertilization in Ax $(1985,1989,1995)$ 
Eh4: internal fertilization $a / p$

E119: external fertilization/direct internal fertilization

Me44: copulation: intradermic/vaginal

Wa29: hypodermic impregnation $\mathrm{a} / \mathrm{p}$

\section{Character scoring and phylogenetic significance}

It is difficult to identify natural suture lines along which to sort the diversity of modes of sperm transfer and fertilization observed in the animal kingdom. Some characters only code mode of sperm transfer, such as Me44 and Wa29, while other combine modes of sperm transfer and fertilization, such as A13 and E119. Each of these coding decisions has its specific strengths and weaknesses.

In the invertebrate zoology literature a common distinction is made between direct and indirect sperm transfer. Direct sperm transfer is restricted to instances of true copulation, in which sperm is directly deposited into the genital openings of the partner, usually through the penetration of a penis. This equates direct sperm transfer (copulation) with internal sperm deposition in the genital system. All other modes of sperm transfer in which contact between sexual partners may be required, but in which gametes are not deposited directly into the genital system are referred to as indirect transfer. These terms are applied to very different phyla. Concrete examples of indirect sperm transfer are hypodermic insemination and dermal impregnation (depositing sperm under the epidermis or on the outside of the body, respectively), as found, for example, in various platyhelminths and onychophorans. Instances of true copulation in which sperm is transferred into the female genital opening is classified as direct sperm transfer in these two phyla (Galleni \& Gremigni, 1989; Ruhberg, 1990). It should be noted that the term 'copulation' is rather artificially restricted to instances of direct sperm transfer or internal sperm deposition. We might as well term indirect modes of sperm transfer "copulation' as long as there is physical contact between the sexual partners (in agreement with the coding of Me44). There is a gray area between direct and indirect modes of sperm transfer, which makes it difficult to characterize some phyla. For example, chaetognaths exhibit a distinctive mating behavior
(Shinn, 1997), which is followed by a so-called pseudo-copulation that does not involve a penetration, but the sperm is nevertheless deposited on the outside of the partner's gonopore through contact between the secual partners (Kapp, 1996; Shinn, 1997). Technically, this mode of sperm transmission should thus be classified as indirect sperm transfer. However, were the sperm deposited on the inside of the gonopore, we would call it direct sperm transfer.

It is difficult to determine primary homology of mode of sperm deposition or transfer in different phyla, because there is much variation in the exact details, even within closely related phyla such as the gastrotrichs, platyhelminths and gnathiferans.

There are two clear alternative character states for mode of fertilization: external and internal. External fertilization refers to fusion of the male and female gametes outside the body of the parent, while internal fertilization refers to fusion of the gametes inside the body of the parent. However, introducing such a simple character with two character states into a phylogenetic analysis of all animal phyla would be grossly misleading. One cannot homologize all instances of internal fertilization found in the Metazoa. There are two reasons for this.

First, mode of fertilization can be quite variable within phyla, and internal fertilization has evidently evolved convergently within well-established monophyletic taxa. Examples can be found in phyla distributed among all the major clades of metazoans such as the cnidarians, molluscs, annelids, priapulids, echinoderms, and urochordates (Haszprunar, 1988; Fautin et al., 1989; Storch, 1991; Rowe et al., 1991; Rice, 1992; Burighel \& Cloney, 1997; Ponder \& Lindberg, 1997). The variation in reproductive modes can be extensive in some cases. For example, massive diversity and probably extensive convergence in reproductive and developmental patterns characterizes the Annelida (Fischer, 1999). A minimum estimate of $25 \%$ of all described polychaete families show more than one mode of fertilization (Glasby et al, 2000). However, this does not preclude, in principle, the possibility of homology of internal fertilization in at least some phyla.

Second, the association of internal fertilization with very different modes of sperm transfer makes 
homology of internal fertilization across the Metazoa exceedingly unlikely. For example, there is little reason to posit primary homology of the internal fertilization found in such divergent groups as the acanthocephalans and the phoronids. Acanthocephalan males use their highly specialized copulatory bursa to attach to a female, after which the male's penis papilla is inserted into the female's vagina for internal sperm transfer and fertilization (Crompton, 1989; Lorenzen, 1996f). In contrast, the phoronids spawn their sperm freely in the seawater. When the sperm, bundled into a spermatophore, comes into contact with a tentacle of another individual, the spermatophore dissolves into a mass of amoeboid sperm that digests the wall of the recipient's tentacle in order to enter into the mesocoelom. For other species of phoronid it appears that sperm is swallowed and then reaches the body coelom through the stomach wall (Zimmer, 1991, 1997). Rather than supporting homology of internal fertilization in these two taxa, it is tempting to speculate that this information suggests independent evolution of internal fertilization as part of reproductive strategies that adapt the acanthocephalans and phoronids to their respective life styles. The acanthocephalans are obligatory endoparasites residing as adults in the digestive system of vertebrates, an environment likely to be unfavorable to the survival and migration of sperm. The phoronids are sessile animals that might boost fertilization success for a modest number of eggs through internal fertilization. Although the data is merely suggestive, the phoronid's closest relatives, the brachiopods, exhibit external fertilization, and appear to spawn larger numbers of eggs (Long \& Stricker, 1991; Zimmer, 1991).

Actual fertilization is a very rarely observed phenomenon in many phyla. Consequently, the determination of mode of fertilization is typically based upon indirect methods. .The presence of internal fertilization may be inferred on the basis of diverse clues such as: 1) sperm morphology, 2) the observation of sperm on, or in, a female/hermaphroditic animal, e.g., entoprocts (Nielsen, 2001), 3) the presence of specialized organs for receiving sperm, such as seminal receptacles, 4) the presence of structures used in copulation, such as male claspers, 5) the observation of the act of copulation, such as in the gnathostomulids (Mainitz, 1989), 6) females releasing developing embryos, e.g., in meiobenthic priapulids (Lemburg \& Schmidt-Rhaesa, 1999), or 7) the observation of fertilization-related physical changes in the oöcyte before it is released from the animal, e.g., formation of a fertilization membrane and commencement of cleavages in placozoan oöcytes (Grell \& Ruthmann, 1991; Ruthmann, 1996). Even the developmental mode of an animal has been used to infer mode of fertilization. For example, direct development is accepted as indicative of internal fertilization in Meiopriapulus (Storch et al., 2000: 16). This interpretation is apparently supported by the ultrastructure of the thread-like sperm in Meiopriapulus (fig. 9A in Storch, 1991) which strongly resembles that of the meiobenthic Tubiluchus for which internal fertilization has been ascertained. Unfortunately, Fig. 9 in Storch (1991) is most likely mislabeled. The text (p. 345,346 ) unambiguously refers to fig. 9 as representing Tubiluchus, and Ahlrichs (1995: 248) additionally noted that the labeling of Storch's fig. 9 contradicts an earlier publication. Thus, unfortunately sperm ultrastructure for Meiopriapulus is still unknown. In conclusion, although the use of indirect criteria to infer fertilization mode is a virtual practical necessity, and although it may yield reasonably reliable results in many groups, it should nevertheless be employed with caution (see Rice, 1992; Rouse \& Pitt, 2000).

Because there is an undeniable correlation between mode of sperm transfer and fertilization, various authors have united both aspects into a single character (e.g. A13, E1 19, and discussions in Ax, 1985, 1989, 1995). Indeed, a key to recognizing the true phylogenetic significance of modes of sperm transfer and fertilization is probably their simultaneous consideration. However, the lack of a perfect correlation introduces difficulties. For example, intuitively one might expect to find a perfect correlation between copulation and internal fertilization on the one hand, and free-spawning of sperm and external fertilization on the other. Indeed, such a distinction is so universally recognized that an almost unanimous attendant assumption prevails about evolutionary polarity, viz., the primitiveness of free-spawning of sperm and external fertilization and the derived condition of direct sperm transfer and internal fertilization (Ax, 1995: 133; Rieger, 
1996a: 203; Biggelaar et al., 1997: 367). However, copulation does not necessarily imply internal fertilization as is illustrated by the oligochaetes (Needham, 1989; Westheide, 1996), and free-spawning does not necessarily imply external fertilization as is illustrated by the poriferans, entoprocts, and phoronids (Fell, 1989, 1997; Emschermann, 1996; Zimmer, 1991, 1997). Moreover, direct sperm transfer and internal fertilization may be primitive for certain taxa (Rouse \& Fitzhugh, 1994; McHugh \& Rouse, 1998), and the hypothesis that these characteristics may also be primitive for supraphyletic clades cannot currently be discounted without argument (Olive, 1985; Buckland-Nicks \& Scheltema, 1995).

Finally, it is instructive to scrutinize and compare the adopted character coding and scoring in the different cladistic analyses. Wa29 and Me44 are restricted to coding mode of sperm transfer only. There are some scoring difficulties associated with Wa29. Gnathostomulida are scored as lacking hypodermic impregnation. However, the filospermoideans probably inject their sperm into the body of the partner (Mainitz, 1989; Sterrer, 1996), while hypodermic impregnation is also a possibility for the scleroperalian bursovaginoids (Mainitz, 1989). Furthermore, the unequivocal scoring of hypodermic impregnation for Rotifera is based upon its presence in monogonont rotifers (Clément \& Wurdak, 1991). The character is inapplicable in the parthenogenetic bdelloids, and it is not found in Seison. Seison mating has not been observed (Gilbert, 1989; Ahlrichs, 1995), but it is likely that the male transfers a spermatophore to the female (possibly into the cloacal opening) using a longitudinal fold of the head region (Ahlrichs, 1995). Pending the resolution of conflicting phylogenies of rotifers and acanthocephalans on the basis of molecular and morphological data (Garey et al., 1996, 1998; Melone et al., 1998; García-Varela et al., 2000; Mark Welch, 2001; Garcîa-Varela et al., 2002; Herlyn et al., 2003), the ground pattern states for Rotifera and Syndermata remain ambiguous. Furthermore, hypodermic impregnation is known in other taxa throughout the Metazoa that were not included in the analysis of Wallace et al. (1996), such as the molluscs, onychophorans, and tardigrades (Bertolani, 1990; Brahmachary, 1989; Ruhberg, 1990).
However, the scattered distribution of hypodermic impregnation in these taxa, and the very variable means by which the process occurs indicate convergent evolution. These include the use of diversely organized penises and penis stylets such as in the rotifers and polychaetes, buccal stylets in the tardigrades, and the lysing of the mate's epidermis after sperm deposition at the body surface in the onychophorans (Bertolani, 1990; Ruhberg, 1990). However, for more restricted sets of phyla, hypodermic impregnation may yet turn out to be a synapomorphy.

Me44 homologizes intradermic and vaginal copulation for the platyhelminths and gnathiferans. Vaginal copulation is supported by the analysis of Melone et al. (1998) as an unambiguous synapomorphy for Syndermata. However, the scoring of Seison as possessing vaginal copulation is not supported by observations (Ahlrichs, 1995), and the unique morphology of the male reproductive system in Seison, including a headfold that functions in the take-up of sperm before transfer to the female, indicates that the mode of sperm transfer is an autapomorphy for Seison. Based on current information, it is most likely that vaginal copulation has independently evolved in the stem lineages of Acanthocephala and within Monogononta where only few species exhibit this character (Hyman, 1951b; Gilbert, 1989). A final problem with Me44 is the scoring of intradermic copulation as the character state for an hypothetical ancestor used for determining character polarity. None of the published comprehensive morphological cladistic analyses support a sister group relationship between the Platyhelminthes and Gnathifera, as is assumed by Melone et al. (1998), and in view of the different out-groups suggested for these taxa in different cladistic analyses the plesiomorphic mode of copulation remains undetermined.

A13, E1 19, and Ax $(1985,1989,1995)$ link mode of sperm deposition and fertilization. This is no problem for Ahlrichs (1995) and the studies of Ax because they considered only restricted sets of taxa. However, one should not indiscriminately unite these features in phylogenetic analyses using a broad range of taxa such as Eernisse et al. (1992) (E119) did. The character states of E119 do not readily complement the taxa selected, i.e., they do not constitute 
clear alternatives that cover the character variation observed in the terminal taxa. In such a situation, misscorings are inevitable (see relevant section under Platyhelminthes for further discussion of character E119).

Finally, the phylogenetic significance granted to internal fertilization (and internal sperm deposition) in Ahlrichs (1995) (synapomorphy for the platyhelminths and gnathiferans), Ehlers et al. (1996) (gastrotrich autapomorphy), and Ax (1989, 1995) (synapomorphy of platyhelminths and gnathostomulids) should be reconsidered as potentially closely related taxa also possess internal fertilization. Internal fertilization is common for cycloneuralians such as the gastrotrichs (Ruppert, 1991b), nematodes (Wright, 1991; Lorenzen, 1996a), nematomorphs (Bresciani, 1991; Schmidt-Rhaesa, 1999), and possibly also the loriciferans and kinorhynchs (Kristensen, 1991a; Kristensen \& Higgins, 1991). Nanaloricus mysticus possesses round-headed sperm, and may exhibit external fertilization which would be unique among loriciferans (R. M. Kristensen, pers. comm., Kristensen \& Brooke, 2002). In fact, macrobenthic priapulids are the only exceptions among aschelminths in possessing external fertilization and a 'primitive' type sperm (generally referred to as "ectaquasperm") (Storch et al., 2000). Schmidt-Rhaesa (1996: 243) argued that the external fertilization and primitive type sperm found in macrobenthic priapulids may represent the plesiomorphic condition for the aschelminths, and that internal fertilization has evolved convergently in the different phyla. However, the diversity of sperm morphologies within the aschelminths currently defies any clear conclusions, since in the analyses of Ax (1989, 1995), Ahlrichs (1995), and Ehlers et al. (1996), the sampling of taxa was too restricted for a proper assessment of the evolutionary significance of mode of fertilization. So far, mode of fertilization has not yet been submitted to a comprehensive computer-assisted cladistic analysis that included all metazoan phyla.

Mode of sperm transfer, copulatory behavior, and mode of fertilization are highly diverse, may vary within phyla, and are functionally linked to each other. These features are not fully congruent, and there are many different morphologies associated with these processes. The issue of primary homol- ogy should be approached with utmost care in future analyses.

\section{Filiform sperm}

Sperm morphology is a highly variable character both within, e.g., the molluscs, platyhelminths, gnathostomulids, and gastrotrichs, and between phyla. However, two general types of sperm are commonly recognized:

(1) sperm with a more or less globular head region, a short middle piece with mitochondria, and a posterior flagellum.

(2) sperm that is thread-like or filiform with a slender head region and elongated nucleus and mitochondria, and a cylindrical middle piece.

The first type of sperm is often designated as primitive and the second as modified. However, the evolutionary significance of these adjectives should be carefully assessed within the context of the taxa studied, and although widespread in the literature, any Metazoa-wide generalizations without recourse to a phylogenetic framework are on shaky ground.

Although variation in sperm morphology has been used as a phylogenetic indicator on lower taxonomic levels, its significance for higher level phylogenetics is less obvious. Nonetheless, a number of recent studies included a character on the absence or presence of filiform sperm to reconstruct metazoan phylogeny (Ax, 1989, Ax, 1995; Eernisse et al., 1992; Ahlrichs, 1995; Zrzavý et al., 1998).

\section{Character coding}

E118; Z117; A13: filiform sperm a/p

G5: filiform sperm without accessory centriole $a / p$

Table 8. Scoring conflicts of filiform sperm.

\begin{tabular}{llll}
\hline & Absent & Present & Proposed scoring \\
\hline Rotifera & Z117 & A13 & Present \\
Acanthocephala & Z117 & A13 & Present \\
\hline
\end{tabular}

\section{Character scoring and phylogenetic significance}

The observed conflict in character scoring illustrates the need for the consistent adoption of an 
exact definition of filiform sperm. Recent analyses agree in scoring the platyhelminths (primarily based upon acoelomorph sperm, Hendelberg, 1983; Watson, 1999; Raikova et al., 2001) and gnathostomulids (based upon filospermoid sperm, Lammert, 1991) as possessing filiform sperm. Accordingly, Ax (1985, 1989, 1995), Eernisse et al. (1992), and Zrzavý et al. (1998) interpreted filiform sperm as an autapomorphy of Plathelminthomorpha (Platyhelminthes and Gnathostomulida). However, Sterrer et al. (1985) hinted that comparison with the sperm of Gastrotricha would be commendable. Later, Ahlrichs (1995) also scored Rotifera and Acanthocephala as possessing filiform sperm and considered filiform sperm as a synapomorphy of Platyhelminthes and Gnathifera. This scoring reflects a character definition that accommodates filiform sperm exhibiting considerable ultrastructural variation, including location of the attachment point of the sperm flagellum on the cell body (posterior if present in plathelminthomorphans, anterior and recurving to posterior in syndermates), possession of a spiral (gnathostomulids) or non-spiral nucleus, and the presence (Seison, gnathostomulids) or absence (monogonont rotifers, most platyhelminths) of acrosome vesicles.

However, it is unlikely that the phylogenetic significance of filiform sperm has been properly resolved in any of the above mentioned phylogenetic studies. There is mounting evidence that the acoelomorphs cannot be used as a representative of the platyhelminth ground pattern as they may be the basalmost extant bilaterians (Ruiz-Trillo et al., 1999, 2002; Baguñà et al., 2001; Jondelius et al., 2002; Telford et al., in press). Also, variation in sperm morphology can be quite extensive within phyla, for example the Gnathostomulida. Mapping the known sperm morphologies (Alvestad-Graebner \& Adam, 1983) on a recent cladogram (Sørensen, 2002) for the Gnathostomulida is at least consistent with regarding filispermoid sperm as plesiomorphic for the group, but the fact that Filispermoida and Bursovaginoidea are sister groups, with diverse sperm types distributed in the latter taxon, at best allows only a tentative conclusion. Filiform sperm is also found in other phyla that are both potentially closely related as well as more distantly related to the platyhelminths and gnathiferans, such as Cycliophora, Entoprocta, Gastrotricha, Myzostomida, Chaetognatha, Onychophora, Arthropoda including Pentastomida, and Pterobranchia (Alvariño, 1983; Franzén, 1983b; Storch, 1993; Storch \& Ruhberg, 1993; Eeckhaut, 1995; Benito \& Pardos, 1997; Shinn, 1997; Funch \& Kristensen, 1997; Nielsen \& Jespersen, 1997; Ruppert, 1991b; Ahlrichs, 1995; Weiss, 2001). These taxa were either incorrectly scored as lacking filiform sperm (Zrzavý et al., 1998), not included in the analysis (Eernisse et al., 1992), or the lack of a numerical phylogenetic analysis did not yield the most parsimonious solution (Ax, 1985; Ax, 1989, Ax, 1995; Ahlrichs, 1995). The morphology of filiform sperm varies quite considerably between different phyla, and needs to be carefully considered if one wants to adopt a character that is meaningfully applicable across the entire Metazoạ.

Franzén (1956) highlighted the link between sperm structure and fertilization biology, and this principle has been widely accepted. Therefore, in order to understand the phylogenetic significance of structural variation in metazoan sperm, we need to gain insight into the functional organization of sperm. Changes in modes of sperm transfer, sperm storage, and fertilization biology are frequently associated with changes in sperm morphology (Franzén \& Rice, 1988), and a filiform sperm morphology with an elongated nucleus and sperm head appears to be one of the most common modifications that evolved convergently within various phyla, including the molluscs (Ponder \& Lindberg, 1996, 1997), annelids (Franzén \& Rice, 1988; Rice, 1992; Kupriyanova et al., 2001), ascidians (Burighel \& Cloney, 1997), priapulids (Storch, 1991; Storch et al., 2000), arthropods (Jamieson, 1987, 1991), nemerteans (Franzén, 1983a; Turbeville, 1991; Stricker \& Folsom, 1998), Lobatocerebrum (Rieger, 1980), hemichordates, based upon presence of filiform sperm in the pterobranch Rhabdopleura, and the possible nesting of the pterobranchs within a paraphyletic Enteropneusta characterized by non-filiform sperm (Cameron et al., 2000; Peterson et al., 2000a; Peterson \& Eernisse, 2001). In most of these cases it is possible to relate the presence of filiform sperm with reproductive modes that appear modified with respect to the presumed primitive mode, in particular copulation and internal fertili- 
zation, but a precise correlation in the context of a phylogenetic framework is necessary for a conclusive determination of character state polarity. In contrast, available information suggests that the presence of filiform sperm is primitive in various other phyla such as the cycliophorans, entoprocts, and chaetognaths. This widespread occurrence has led some authors to suggest homology of all filiform sperm across the Bilateria (Buckland-Nicks \& Scheltema, 1995). However, the phylogenetic significance of this widespread distribution of filiform sperm remains to be carefully assessed. Despite a common name, filiform sperm covers a range of ultrastructural variation, and the undeniable relationship between sperm morphology and fertilization biology makes determination of primary homology very difficult. Furthermore, studies of sperm ultrastructure in taxa such as the acoels show that sperm morphology may be quite variable even between closely related congeneric species (Raikova et al., 2001).

At present, the pattern of evolution of sperm morphology across the Metazoa remains unresolved. Some remarkable similarities in the morphology of filiform sperm have undoubtedly evolved convergently, as for example the peculiar V-shaped filiform sperm found in phoronids and the unrelated, morphologically highly modified echinoderm $X y l o p l a x$, in which the centriolar apparatus is located anterior in the sperm with a flagellum recurving along the sperm body (Rowe et al., 1991; Zimmer, 1991). Interestingly, this feature received recent recognition as a unique synapomorphy of a clade of Cycliophora, Myzostomida and Syndermata, which was named Prosomastigozoa after the anterior insertion point of the sperm flagellum (Zrzavý et al., 2001: character ZI38). Previous phylogenetic studies proposed this type of sperm as unique for the rotifers and acanthocephalans (Wallace et al., 1996; Ahlrichs, 1997; Zrzavý et al., 1998; Kristensen \& Funch, 2000; Sørensen et al., 2000: characters Wa38, Ah5, Z121, K7, and S25 respectively). Brusca \& Brusca (2003) also only score this feature as present in the syndermates (BI25). However, none of these studies included or scored this feature as present for phyla such as the Phoronida and Chaetognatha, which possess strikingly similar sperm (Zimmer, 1991; Alvariño, 1983).
Spermatozoa without accessory centriole

The most common situation for metazoan sperm (also typical of other metazoan cells possessing motile cilia) is the possession of a diplosome comprising the basal body of the cilium and an accessory centriole which are typically oriented perpendicular to each other. Various taxa lack an accessory centriole in their sperm cells, notably syndermates.

\section{Character coding}

P19; ZI39; A13; ZII83: spermatozoa without accessory centriole $\mathrm{a} / \mathrm{p}$

G5: filiform sperm without accessory centriole $\mathrm{a} / \mathrm{p}$

Table 9. Scoring conflicts for spermatozoa lacking an accessory centriole.

\begin{tabular}{|c|c|c|c|}
\hline & $?$ & $\begin{array}{l}\text { Absent } \\
\text { (acc. } \\
\text { centriole } \\
\text { present) }\end{array}$ & $\begin{array}{l}\text { Present } \\
\text { (acc. } \\
\text { centriole } \\
\text { lacking) }\end{array}$ \\
\hline Cycliophora & P19; ZII83 & & ZI39 \\
\hline Catenulida & ZI39; ZII83 & & P19 \\
\hline Rhabditophora & ZI39; ZII83 & & P19 \\
\hline Annelida & ZI39; ZII83 & P19 & \\
\hline Chaetognatha & ZI39; ZII83 & P19 & \\
\hline Gastrotricha & ZI39; ZII83 & P19 & \\
\hline Entoprocta & ZI39; ZII83 & P19 & \\
\hline Ectoprocta & ZI39; ZII83 & P19 & \\
\hline Onychophora & ZI39; ZII83 & P19 & \\
\hline Phoronida & ZI39; ZII83 & P19 & \\
\hline Tardigrada & ZI39; ZII83 & P19 & \\
\hline Gnathostomulida & ZI39; ZII83 & & P19; A13 \\
\hline Nematoda & $\mathrm{ZI} 39$ & P19; ZII83 & \\
\hline Nematomorpha & Z139 & P19; ZII83 & \\
\hline
\end{tabular}

Character scoring and phylogenetic significance

Zrzavý et al. (2001) and Peterson \& Eernisse (2001) exhibit a substantial number of scoring conflicts. However, in an unpublished revised version of the data matrix of Peterson \& Eernisse (2001) all these scoring conflicts with the exception of Cycliophora and Nematoida, are resolved in favor of the scoring of ZI39 (K: J. Peterson, pers. comm.). I have not comprehensively studied the distribution of 
sperm accessory centrioles in the Metazoa.

The scoring of spermatozoa lacking an accessory centriole in Cycliophora for ZI39 is puzzling considering the character discussion provided. Zrzavý et al. (2001) clearly state that there is no information available on the fine structure of cycliophoran sperm, yet they base their scoring on the lack of accessory centrioles in ciliated cells of the buccal region. Obviously, this is an unjustifiable rationale for scoring sperm ultrastructure.

The nematodes, nematomorphs, and bdelloid rotifers are scored 'inapplicable' of "absent" for accessory centrioles (ZI39; ZII83). This is in agreement with available data for nematomorphs and bdelloids, but not necessarily for nematodes. No centrioles have been observed at all in the aflagellate nematomorph sperm (Bresciani, 1991; SchmidtRhaesa, 1996), and no sperm known for bdelloids. However, a pair of centrioles can usually be discerned in the aflagellate nematode sperm (Wright, 1991), which might justify the scoring of an accessory centriole. In any case, there appears to be little basis for proposing that a lack of an accessory centriole in the nematodes, nematomorphs, and bdelloid rotifers is homologous. These taxa show a pair of centrioles in aflagellar sperm, no centrioles in aflagellar sperm, or no sperm at all, respectively. No. attempt was here made to verify the scoring for the other phyla.

\section{Sperm flagella}

\section{Character coding}

Z115: sperm flagella monoflagellate/aflagellate/ biflagellate

Wa37: sperm flagellum a/p

\section{Character scoring and phylogenetic significance}

No scoring conflicts were observed for Z115 and Wa37. The presence of monociliated sperm is evidently the plesiomorphic character state for the Metazoa, but a fair number of changes towards biflagellate or aflagellate sperm have been described for different phyla. The total lack of sperm flagella in the nematodes and nematomorphs may support a monophyletic Nematoida. The patterns of distribu- tion of other instances of aflagellate sperm are suggestive of convergent evolution. For example, aflagellate sperm is known in catenulid and macrostomid platyhelminths, rhombozoans (all scored accordingly in Z115), and it also has apperently evolved independently within the gastrotrichs (Ruppert, 1991b), kinorhynchs (Kristensen \& Higgins, 1991), and arthropods (Jamieson, 1991). The biflagellar sperm found within the platyhelminths evolved independently from that which is found within the arthropods (Jamieson, 1987).

\section{Gonads asacular or sacular}

Haszprunar (1996a) introduced a character coding for the organization of gonads into a cladistic analysis of a subset of metazoan phyla (H31). A distinction was made between sacular and asacular gonads, following the definition proposed in Rieger et al. (1991b; see also Rieger, 1996a). Sacular gonads are defined on the basis of a somatic cellular lining (mesodermal according to Rieger, 1996a) surrounding the germ cells. Asacular gonads lack such a lining, and may thus not be well-defined organs. In this case, the germ cells mature between the body wall and gastrodermis, often close to the base of the latter.

\section{Character coding}

H31; Z110: gonads asacular/sacular in males/sacular in both sexes

HaVIIb: testis sacular $a / p$

ZII89: sacular gonads a/p

\section{Character scoring and phylogenetic significance}

H31 and Z110 both misscored the rhabditophorans. Rieger et al. (1991b) clearly report that sacular gonads are typically found in virtually all the rhabditophorans, except for a few of the prolecithophorans. Furthermore, in contrast to the scoring of Z110, available ultrastructural data for Cycliophora does not allow a clear characterization of their gonad organization (Funch \& Kristensen, 1997). A few instances of variation for the presence of gonadal somatic lining cells are known, e.g., within the nematodes the male of Caenorhabditis possesses gonads without somatic lining cells but instead dis- 
plays a basal lamina that surrounds the testis, and the ovary of the gastrotrich Lepidodermella squamata lacks lining or duct cells (Wright, 1991; Ruppert, 1991b). However, these abberant conditions are of little relevance to the ground pattern states for these phyla.

Rieger et al. (1991b) considered diffuse asacular gonads to represent the primitive metazoan condition, as exemplified by certain cnidarians, acoelomorphs, most catenulids, and some prolecithophorans (derived rhabditophorans). Sacular gonads were thought to be characteristic of virtually all the rhabditophorans, and other non-coelomate phyla (Rieger et al., 1991b; Rieger, 1996a). Coelomate animals were considered as a separate category of gonad organization. However, Haszprunar (1996a) instead scored sacular gonads as present in the coelomates. In his study with a limited sampling of phyla he found sacular gonads to be a synapomorphy for Nemertea, Myzostomida, and Trochozoa. However, the broader taxon sampling in Zrzavý et al. (1998) and Zrzavý (2003) showed that sacular gonads may be a synapomorphy for all of the bilaterians, with subsequent reversals to an asacular condition in several taxa such as the acoelomorphs, catenulids, gnathostomulids, seisonid rotifers (Ahlrichs, 1995), and Lobatocerebrum (the subsequently described Limnognathia [Micrognathozoa] also lacks gonadal somatic lining cells: Kristensen \& Funch, 2000).

It should be noted that the character scoring in Zrzavý et al. (1998) and Zrzavý (2003) assumes homology of all organized gonads irrespective of the presence or nature of their lining. Thus sacular gonads are scored as present for taxa with real cellular gonadal linings, such as the coelomate phyla, and taxa lacking cellular linings, such as Seison annulatus (fig. 48 in Ahlrichs, 1995). The ovaries of the Micrognathozoa also lack any cellular lining, and appear to be solely made up of the oocytes themselves (Kristensen \& Funch, 2000). A detailed ultrastructural comparison of gonad morphology in the coelomates and non-coelomates may reveal additional phylogenetic characters.

\section{Gonocoel}

The gonocoel is the lumen of the gonad. Defined in this way, without taking the ultrastructure of the gonadal lining (acellular or cellular) into account, a gonocoel can be identified in virtually all bilaterians that possess sacular gonads (excluding the acoelomorph and catenulid platyhelminths). However, the character definitions adopted in recent cladistic analyses consider gonocoels to be restricted to gonads with a cellular lining, or more precisely, to coelomate taxa with retroperitoneal gonads.

\section{Character coding}

$\mathrm{H} 27$; Z31: gonocoel (retroperitoneal gonads) a/p E20: gonocoel (coelom reduced to perigonadal region) $\mathrm{a} / \mathrm{p}$

ZI44: retroperitoneal gonads with gonocoel $a / p$

Table 10. Scoring conflicts for gonocoel.

\begin{tabular}{llll}
\hline & Absent & Present & $?$ \\
\hline Nemertea & H27 & Z31 & ZI44 \\
Arthropoda & ZI44 & & Z31 \\
Tardigrada & ZI44 & Z31 & \\
Onychophora & ZI44 & Z31 & \\
\hline
\end{tabular}

Character scoring and phylogenetic significance

So far, four different cladistic analyses have included a character on the distribution of gonocoels within the Metazoa (Eernisse et al., 1992; Haszprunar, 1996a; Zrzavý et al., 1998, 2001). Unfortunately, none of these analyses has yielded any meaningful insight into the evolutionary origin or diversification of gonad cavities.

Eernisse et al. (1992) defined a gonocoel as a coelom reduced to the perigonadal region. This character definition a priori introduces an unwarranted assumption about character polarity. E20 was only scored for molluscs and panarthropods, which are sometimes assumed to have reduced coeloms, although this assumption is by no means supported by all present phylogenetic hypotheses. When a more rigorous structural definition for a gonocoel is applied, for example a cavity lined by a cellular epithelium or coelom, we find no special similarity at all that would justify the exclusive scoring for molluscs and panarthropods in the analysis of Eernisse et al. (1992). Other coelomate taxa included in the study would than have to be scored for pres- 
ence of a gonocoel as well, including the remaining coelomate protostomes, lophophorates, and deuterostomes.

Haszprunar (1992, 1996a) proposed that the gonocoel is a synapomorphy of the Mollusca and Eucoelomata which he defined as all protostome and deuterostome coelomate phyla, and the lophophorates. Restrictive taxon sampling in Haszprunar's (1996a) analysis (leaving out all eucoelomate phyla except Sipuncula, Echiura and Annelida) did not allow him to test the hypothesis that a gonocoel is a synapomorphy of the eucoelomates. The broader taxon sampling in the analysis of Zrzavý et al. (2001), which included the lophophorates and deuterostomes, in principle allowed this hypothesis to be tested, but instead Zrzavý et al. (2001) simply adopted the same scoring as Haszprunar (1996a), i.e., only the neotrochozoan phyla were scored as having a gonocoel. Zrzavý et al. (1998) did score Nemertea, Tardigrada and Arthropoda in addition to neotrochozoans, but they failed to score the remaining coelomate phyla.

It is interesting that both E20 and Z31 score the nemerteans and tardigrades as possessing gonocoels as well. This indicates that a gonocoel is present in all taxa with complete (Nemertea: Turbeville, 1991) or incomplete (Tardigrada; Dewel et al., 1993) cellular gonadal linings (without distinguishing between germinal and somatic lining cells). Adoption of such a character definition would necessitate rescoring many other phyla in addition to the coelomate lophophorates and deuterostomes, including the rhabditophoran platyhelminths (Rieger et al., 1991b), entoprocts (Nielsen \& Jespersen, 1997), Lobatocerebrum (only in males; Rieger, 1980), nematodes (Wright, 1991), nematomorphs (at least Gordiida; Lanzavecchia et al., 1995; SchmidtRhaesa, 1999), and priapulids (Storch, 1991). For all these groups cellular gonadal linings have been reported. Obviously, a thorough restudy (including the confirmation of light microscopical information in taxa such as the platyhelminths and Lobatocerebrum) and rescoring of gonocoel characters is required to assess the variation present in the animal kingdom. Only then can we study the evolutionary relationship between gonads with and without (presumed mesodermal) cellular linings.

Additionally, scoring presence of a gonocoel for taxa with diverse body cavity organizations suggests primary homology of the gonadal cavities of non-coelomate taxa such as the platyhelminths, both with the restricted coelomic cavities such as present in the nemerteans and molluscs, and with the relatively spacious body coeloms of taxa such as the annelids and sipunculans. This proposal of homology would revive what was thought to be a largely settled debate about the gonocoel theory, a key ingredient of which is the homology of all histologically defined coeloms, including gonocoels and body coeloms (Goodrich, 1895). This theory was proposed in the late $19^{\text {th }}$ century and was advocated by various zoologists. Although some recent commentaries on it have been more or less positive (Kozloff, 1990; Salvini-Plawen, 1998a), it is currently most frequently dismissed as having "lost its explanatory power" (Bartolomaeus, 1999: 23; Willmer, 1990; Meglitsch \& Schram, 1991).

\section{Development}

\section{Spiral cleavage}

Spiral cleavage is a stereotypical cleavage pattern that is characterized by an oblique orientation of the mitotic spindles with respect to the primary (animal-vegetal) egg axis. The spindle orientations alternate in successive cleavage divisions creating tiers of blastomeres with alternating orientations relative to each other (see Boyer \& Henry, 1998 and Henry \& Martindale, 1999 for a discussion of the ontogenetic and evolutionary variations upon the theme of spiral cleavage). Importantly, spiral cleavage produces blastomeres with closely similar developmental fates between taxa, as is convincingly illustrated by corresponding cell lineages in the neotrochozoans. Two forms of spiral cleavage are usually recognized in recent cladistic analyses: "general" spiral cleavage which is scored for a broad range of taxa and spiral quartet cleavage, which is a subcategory of the first type, and which is generally considered lacking in acoelomorph platyhelminths. 
Table 11. Scoring conflicts for spiral cleavage.

\begin{tabular}{|c|c|c|c|c|}
\hline & Absent & Present & $?$ & Proposed scoring \\
\hline Nematoda & $\begin{array}{l}\mathrm{P} 29, \mathrm{Z} 6, \mathrm{Z7}, \mathrm{ZI} 1, \mathrm{ZII} 2 \\
\mathrm{E} 1, \mathrm{Wa} 31, \mathrm{M} 18, \mathrm{BI} 18\end{array}$ & E2 & & Absent \\
\hline Arthropoda & $\mathrm{Z6}, \mathrm{Z7}, \mathrm{ZI1}, \mathrm{ZII} 2, \mathrm{P} 29$ & $\begin{array}{l}\text { E1 (Crustacea), } \\
\text { E2 (Crustacea), } \\
\text { M18, B118 }\end{array}$ & & Absent \\
\hline Rotifera & $\mathrm{Z6,} \mathrm{Z7,} \mathrm{ZI1}, \mathrm{M} 18$ & Wa31, BI18 & P29, ZII2 & Absent \\
\hline Acanthocephala & $\mathrm{Z6}, \mathrm{Z7}, \mathrm{ZI1}, \mathrm{M} 18$ & Wa31, BI18 & ZII2 & Absent \\
\hline Acocla & $\begin{array}{l}\text { P29 (quartet), Z7, ZI1 and } \\
\text { ZII2 (quartet),H33 (quartet } \\
\text { for acoelomorphs), E1 } \\
\text { (quartet for acoelomorphs) }\end{array}$ & $\begin{array}{l}\text { Z6 (duet), H32 } \\
\text { (duet for } \\
\text { acoelomorphs), E2 } \\
\text { (acoelomorphs) }\end{array}$ & & Absent \\
\hline Catenulida & & Z6, Z7, ZI1, ZII2, P29 & $\mathrm{H} 32, \mathrm{H} 33$ & Present \\
\hline Pogonophora & $\mathrm{E} 1$ (quartet), $\mathrm{Z7}$ (quartet) & $\mathrm{E} 2, \mathrm{C} 11, \mathrm{RI} 1, \mathrm{M} 18$ & $\mathrm{Z} 6$ & Present \\
\hline Kinorhyncha & 27 & & $\begin{array}{l}\mathrm{Z} 6, \mathrm{ZII} 2, \mathrm{P} 29, \mathrm{Wa} 31 \\
\mathrm{M} 18, \mathrm{E} 1, \mathrm{E} 2, \mathrm{BI} 18\end{array}$ & $?$ \\
\hline Loricifera & man & & $\begin{array}{l}\mathrm{Z} 6, \mathrm{ZII} 2, \mathrm{P} 29, \mathrm{Wa} 31 \\
\mathrm{M} 18, \mathrm{BI} 18\end{array}$ & $?$ \\
\hline Nematomorpha & $\begin{array}{l}\mathrm{P} 29, \mathrm{Z} 6, \mathrm{Z} 7 \text { and } \mathrm{ZII} 2 \text { (quartet), } \\
\mathrm{Wa} 31, \mathrm{M} 18, \mathrm{BI} 18\end{array}$ & & ZIl (quartet) & Absent \\
\hline Tardigrada & $\mathrm{Z7}, \mathrm{P} 29$ & $\mathrm{M} 18, \mathrm{BI} 18$ & Z6, ZI1, ZII2, E1, E2 & $?$ \\
\hline Onychophora & Z6, Z7, ZI1, P29, ZII2 & $\mathrm{M} 18, \mathrm{BI} 18$ & $\mathrm{E} 1, \mathrm{E} 2$ & Absent \\
\hline Gnathostomulida & & $\begin{array}{l}\text { Z6, Z7, ZI1, ZII2, E2, } \\
\text { Wa31, H32, H33, M18, } \\
\text { P29, BI18 }\end{array}$ & E1 (quartet) & Present \\
\hline Choanoflagellata & P29 & & $\mathrm{Z6}, \mathrm{Z7}$ & Inapplicable \\
\hline
\end{tabular}

\section{Character coding}

P29; Z6; Eh2; G4: spiral cleavage a/p

Z7; ZI1; ZII2; E1: spiral quartet cleavage $a / p$

H32; Wa31: non-spiral cleavage/spiral cleavage

H33: spiral quartet cleavage $a / p$

E2: spiral cleavage with nuclear migration $a / p$

B13b: typical spiral cleavage $a / p$

Ca3: cleavage spiral/radial or biradial

C11: cleavage spiral/radial

M18: cleavage radial/spiral quartet cleavage

N20; NI18; S26: spiral cleavage with 4d-mesoderm $\mathrm{a} / \mathrm{p}$

Hald; L52: spiral cleavage with two or four quartets of micromeres and mesoderm out of micromere $2 \mathrm{~d}$ or $4 \mathrm{~d}$ (or probably derived from this pattern in L52) $a / p$

BI18: cleavage fundamentally spiral/fundamentally $\mathrm{radial} / \mathrm{neither}$ clearly spiral nor radial/unique ctenophoran cleavage

\section{Character scoring and phylogenetic significance}

Nematodes clearly do not have spiral cleavage (Lorenzen, 1996a; Nielsen, 2001), contra E2, Costello (1955), and Anderson (1981).

Reports of spiral cleavage in Arthropoda (even Panarthropoda) are restricted to some crustaceans, in particular the cirripedes (e.g. Anderson, 1969; Costello \& Henley, 1976), and pycnogonids (e.g. Anderson, 1973; Nielsen, 2001). However, I follow the conclusions of Scholtz's (1997) detailed discussion and regard the presence of spiral cleavage in the arthropod ground pattern as unlikely (although not definitely disproven), in agreement with the scoring of Zrzavý et al. (1998, 2001), Zrzavý (2003) and Peterson \& Eernisse (2001). Recent cell lineage studies of amphipod and decapod crustaceans also do not support the presence of spiral cleavage in arthropods (Gerberding et al., 2002; Hertzler, 2002; Scholtz \& Wolff, 2002; Wolff \& Scholtz, 2002). 
The scoring of spiral cleavage for the rotifers in Wallace et al. (1996) and Brusca \& Brusca (2003) is not without precedent, but the current literature showcases a wide range of interpretation of rotiferan cleavage, from spiral cleavage (Wallace et al., 1996), through "modified" spiral cleavage (Costello, 1955; Costello \& Henley, 1976; Anderson, 1981; Gilbert, 1989) to radial cleavage (Valentine, 1997). This clearly illustrates that the early cleavage pattern of rotifers is very difficult to interpret and to characterize either as radial or spiral cleavage. Siewing (1979) argued against the interpretation of rotiferan cleavage as spiral or "modified" spiral primarily because the D quadrant in rotifers contributes chiefly to the ventral side of the body, in contrast to spiralians where the D quadrant denotes the dorsal side of the body (see also Table 31.1 in Nielsen, 2001). Initially, Nielsen (1995) rejected the comparison of the rotiferan cleavage pattern with spiral cleavage, but later Nielsen (2001) suggested that the four cleavage quadrants of rotifers are reminiscent of the four quadrants of the spiral quartet cleavage of spiralians. However, he noted at the same time that "the spiral pattern is lacking" (Nielsen, 2001: 300). The interpretation of rotiferan cleavage as "modified" spiral appears to hinge upon the phylogenetic position of Rotifera among spiral cleaving phyla, which is a possibility but by no means certain. I therefore tentatively conclude that the scoring of Zrzavý et al. $(1998,2001)$ and Peterson $\&$ Eernisse (2001) are both supported by available data in contrast to the scoring of Wallace et al. (1996).

A similar uncertainty exists about the cleavage pattern of the acanthocephalans, which has led some authors to conclude that they exhibit a "distorted" spiral cleavage pattern (Crompton, 1989). The unambiguous scoring of a clear spiral pattern for the acanthocephalans (Wa31, BI18) is wrong (Crompton, 1989; Nielsen, 2001). Early syncytialization of the embryo and cell migrations are not particularly indicative of spiral cleavage, warranting the scoring of Zrzavý et al. $(1998,2001)$ versus Wallace et al. (1996).

Duet cleavage has been clearly documented for acoels (Boyer et al., 1996b; Boyer \& Henry, 1998; Henry \& Martindale, 1999; Henry et al., 2000; Nielsen, 2001). Until recently this highly distinc- tive cleavage pattern was almost universally interpreted as a form of modified spiral quartet cleavage, at least until recent molecular and morphological cladistic analyses suggested the possibility that the acoels as well as the nemertodermatids might constitute the basal-most bilaterian crown taxa (Haszprunar, 1996a, b; Ruiz-Trillo et al., 1999, 2002; Baguñà et al., 2001; Jondelius et al., 2002; Telford et al., in press). The interpretation of duet cleavage as related to spiral quartet cleavage becomes much less plausible with this basal placement of the acoelomorphs. It is quite obvious that the presumed phylogenetic placement of the acoels has had a considerable effect upon the interpretation of their cleavage as a modified spiral quartet type. This is clearly revealed by considering the change of opinion in the studies of acoel development by B. C. Boyer, J. Q. Henry and M. Q. Martindale from 1996 to 2000. The turning point occurred in 1999 when RuizTrillo et al. (1999) published their remarkable 18S rDNA phylogeny that suggested that acoels were not closely related to the other platyhelminths, but instead represented the earliest diverging of extant bilaterian lineages. This proposition conformed to some earlier ideas based upon phylogenetic analysis of morphological data by Haszprunar (1996a, b). The papers up to and including 1999 (Boyer et al., 1996b; Boyer \& Henry, 1998; Henry \& Martindale, 1999) all reported that duet cleavage could be interpreted as modified spiral quartet cleavage by focusing on potential similarities with this cleavage type. In contrast to this, Henry et al. (2000) emphasized the distinctness of acoel duet cleavage and additionally suggested that certain features were perhaps more reminiscent of early development in diploblasts (in particular ctenophores). In view of continuing controversy about the placement of acoels within the Metazoa, it is therefore reasonable to expect that the debate about the evolutionary origin of acoel cleavage will continue. It should also be noted that although some workers score the nemertodermatids as having spiral cleavage, e.g., Littlewood et al. (1999a: L52), I could not trace any reliable published account, and Nielsen (2001) relies on a personal communication from $\mathrm{O}$. Israelsson as authority for duet cleavage in this taxon (see also Telford, 2001:261). If acoels and nemertodermatids are sister taxa, this leaves the cleavage pat- 
tern in the acoelomorph ground pattern uncertain.

Although catenulid development is very poorly documented, they appear to have spiral quartet cleavage (Thomas, 1986; Rieger, 1996c). However, modern investigations are badly needed to confirm the few early reports from the mid-1900s by E. Reisinger and S. Bogomolow (I have not been able to consult these papers).

The Pogonophora (including Vestimentifera) provide another instructive example of how phylogenetic speculations can color character interpretations (see Rouse \& Fauchald, 1995 for the rather tumultuous systematic history of the pogonophorans). Rouse \& Fauchald (1995: 272) think that Ivanov's early reports of modified radial cleavage in pogonophores "may have been an interpretation based on expectation" because of the supposed affinity of the pogonophores with hemichordates. Later Ivanov (1988) reported modified spiral cleavage at the same time as he advocated a phylogenetic affinity to both protostomes and deuterostomes. This is in line with the interpretation of modified spiral cleavage reported in most recent papers (Ivanov, 1988; Bakke, 1990; Southward, 1999; Salvini-Plawen, 2000; Nielsen, 2001).

The nature of embryonic development of both the loriciferans and kinorhynchs remains unknown to date, contra Z7 (Nielsen, 2001). Note that these taxa are also misscored in table 2.3 in Hall (1999), which summarizes the distribution of metazoan cleavage patterns. Other taxa in Hall's table for which spiral cleavage was incorrectly scored include the poriferans, ctenophores, cycliophorans, gastrotrichs, and acanthocephalans.

Various geometries have been proposed for nematomorph cleavage, recently including modified spiral cleavage (Brusca \& Brusca, 1990), radial cleavage (Zrzavý et al., 1998: Z5), or modified radial cleavage (Valentine, 1997 after Bresciani, 1991; I have failed to trace the source of this information in Bresciani, 1991). Schmidt-Rhaesa (1999) suggests that the 4-cell stage embryo is similar to those of various nematodes and gastrotrichs, in which two pairs of two cells are positioned in planes perpendicular to each other (see also Nielsen, 2001: 97). However, after the 4-cell stage, the blastomeres do not cleave simultaneously. The scoring of the nematomorphs as lacking 'unmodified' spiral cleav- age in Wallace et al. (1996), Zrzavý et al. (1998), Zrzavý (2003), Brusca \& Brusca (2003), and Peterson \& Eernisse (2001) appears to be justified.

In the only modern study of early tardigrade embryology, Eibye-Jacobsen (1996/1997) believed that the cleavage pattern may be a modified spiral type. However, the data is not compelling, and all recorded scorings in the literature can currently be defended.

Although E1 and E2 scored a '?' for spiral cleavage in the Onychophora and M18 and BI18 scored it present, cleavage has been described for a number of species, without revealing any traces of spiral cleavage (Anderson, 1973; Ruhberg, 1990; Nielsen, 2001), thus justifying the scoring in Zrzavý et al. (1998, 2001), Zrzavý (2003), and Peterson \& Eernisse (2001).

It is perhaps surprising to learn that the widely adopted inclusion of gnathostomulids in the Spiralia is primarily based upon a sole report of spiral cleavage from more than 30 years ago by Riedl for Gnathostomula jenneri (Riedl, 1969; Mainitz, 1989). Nevertheless, spiral quartet cleavage was observed, in contrast to the scoring for E1.

Because the colonial choanoflagellates do not develop through embryonic cleavage stages, they should be scored as 'inapplicable' in agreement with Z6 and Z7, and in contrast to P29.

Zrzavý et al. (1998) included mesozoans in their analysis, and they scored spiral cleavage for rhombozoans. Although the early cell divisions indeed appear to be consistent with a spiral cleavage pattern, it is more difficult to characterize the later cleavages (see figs. 3 and 4 in Furuya et al., 1992). However, the molecular phylogenetic information from 18S rRNA and 18S rDNA sequences, as well as a characteristic Hox gene peptide motif, indicate that dicyemid rhombozoans are bilaterians, and possibly lophotrochozoans that are closely related to phyla with spiral cleavage (Katayama et al., 1995; Pawlowski et al., 1996; Peer \& Wachter, 1997; Kobayashi, Furuya \& Holland, 1999; see also Telford, 2000). This would buttress the interpretation of their cleavage as of the spiral quartet type.

N20, NI18, S26, L52, and HaId combined cleavage pattern and source of mesoderm into one complex character. This combination appears to be justified for the trochozoans, where mesoderm is 
typically derived from the $4 \mathrm{~d}$-cell. However, this combination leads to erroneous scorings for some other taxa. First, even accepting spiral cleavage in the arthropod ground pattern (but see above), the sources of mesoderm are not in agreement with those in the trochozoans, i.e., not from mesentoblast $4 \mathrm{~d}$ (Anderson, 1973; Siewing, 1979; Scholtz, 1997; Nielsen, 2001). The scorings of N20 and S26 for arthropods should be changed from ' 1 ' to ' 0 ' accordingly. Second, although spiral quartet cleavage may be accepted for Gnathostomulida, their later development, including the source of mesoderm, has not yet been traced. NI18, S26, and L52 should be rescored from ' 1 ' to '?' accordingly. Finally, the scoring of a '?' for S26 in the ctenophores is incorrect. Recent cell lineage tracing studies have unequivocally established the unique cleavage program of ctenophores, with no similarity in either the cleavage pattern or the cell fates (including mesoderm) to spiral cleavage (Martindale \& Henry, 1999).

If we study the distribution of spiral (quartet) cleavage on the most recent comprehensive morphological phylogenies that included this character, some interesting conclusions emerge. First, a single evolutionary origin of spiral cleavage is supported by Eernisse et al. (1992), Nielsen et al. (1996), Nielsen (2001), Zzravy et al. (1998, 2001); Zrzavý (2003), Sørensen et al. (2000), and Peterson $\&$ Eernisse (2001), with two possible exceptions. The spiral cleavage reported for Rhombozoa may have independently evolved (Zrzavý et al., 1998), but in view of the continuing uncertainty about their phylogenetic position, which may be among other spirally cleaving phyla (Kobayashi et al., 1999), a definitive conclusion is not yet possible. The second possible instance of independent evolution of spiral cleavage was suggested by the analysis of Zrzavý et al. (2001) for the gnathostomulids. In their analysis, the gnathostomulids grouped together with the gastrotrichs to form a clade Neotrichozoa (the total evidence analysis of Zrzavý et al., 1998 and 2001 also supported this grouping, in contrast to the morphological analysis of Zrzavý et al., 1998) that formed the sister group to the remaining protostomes. However, as is discussed in detail under Gnathostomulida, the morphological character support for this clade is limited to protonephridial fea- tures, which exhibit a variety of scoring problems. Moreover, the synapomorphies that group all protostomes except gnathostomulids and gastrotrichs are also not compelling (see under Gnathostomulida). We can thus conclude that the probability of the gnathostomulids independently having evolved spiral cleavage from the remaining spiralians is not high.

Second, the phylogenetic positions of several phyla that have cleavage patterns that are difficult to interpret suggest that they indeed may have modified plesiomorphically present spiral cleavage. These include the ectoprocts (Nielsen et al., 1996; Nielsen, 2001; Peterson \& Eernisse, 2001), panarthropods (Nielsen et al., 1996; Sørensen et al., 2000; Nielsen, 2001), and syndermates (Sørensen et al., 2000; Nielsen, 2001; Zrzavý et al., 2001). However, reports of "modified" spiral cleavage patterns in the gastrotrichs and nematodes receive no support through their phylogenetic placement. The phylogenetic significance of reports of early spiral-like cleavages in the hydrozoans, scyphozoans and anthozoans (Uchida \& Yamada, 1968; SalviniPlawen, 1978) are uncertain at best. A variety of cleavage patterns is found within the Cnidaria, but although some species exhibit invariant cleavage geometries, the plesiomorphic state may rather be more or less "chaotic" cleavage, i.e., a kind in which no regular cleavage pattern can be discerned, even between embryos from the same spawning (Davidson, 1991; Martindale \& Henry, 1998; Martin, 1997). Whatever the precise ancestral cleavage pattern, regular spiral cleavage is unknown for the cnidarians: Similarly, the phylogenetic significance of the recently described hexactinellid spiral cleavage (Boury-Esnault et al., 1999) is doubtful.

\section{Blastula}

A blastula stage of development can be recognized in members of all metazoan phyla. It represents the developmental stage before the germ layers are formed. Two types of blastulae are commonly recognized in animals with eggs containing little to moderate amounts of yolk, a coeloblastula, and a sterroblastula (also called stereoblastula). The coeloblastula is hollow, with a central blastocoel surrounded by, in its simplest form, a single cell 
layer thin epithelium. A sterroblastula is solid, lacking a blastocoel. Generally one finds coeloblastulae in taxa that exhibit radial cleavage, whereas taxa with spiral cleavage frequently, but not exclusively, display sterroblastula.

\section{Character coding}

P27: blastula stage $a / p$

Z11: coeloblastula $\mathrm{a} / \mathrm{p}$

\section{Character scoring and phylogenetic significance}

P27 scores all metazoan taxa for which there is evidence of the early embryological stages as having a blastula, in agreement with the universal interpretation of the blastula stage as a shared character of all Metazoa.

Zrzavý et al. (1998) introduced a character on the presence of a coeloblastula for sorting animal relationships. Most non-bilaterians and all deuterostomes are scored to possess a coeloblastula, while many protostomes received a '?.' The phylogenetic significance of this character appears to reside mainly in the support it provides for several taxa that have supposedly lost a hollow blastula, namely Silicispongea (demosponges and hexactinellids), Syndermata, and Platyhelminthes (nemertodermatids and catenulids were scored '?'). Several comments on scoring and character definition are in order.

Although it is true that silicisponges typically form a solid blastula stage, a coeloblastula has been observed in various demosponges (Fell, 1989; Harrison \& De Vos, 1991). Similarly, Z11 scores rotifers as unambiguously lacking coeloblastulae, but a small blastocoel has at least been observed during parthenogenetic development in the monogonont rotifer Asplanchna (fig. 3.1 in Gilbert, 1989). This hints at an important aspect of blastula formation throughout different phyla: it is highly variable with a resulting uncertainty about the ground pattern states of many groups.

A major determinant of variation in early ontogenetic processes such as blastulation and gastrulation is the amount and location of yolk in the eggs, as is clearly illustrated by the divergent early embryogenesis of many arthropods, cephalopod molluscs, and amniotes with yolk-rich eggs, e.g., Arendt
\& Nübler-Jung (1999b). It is therefore not surprising to discover that many phyla with holoblastic cleavage exhibit both coelo- and sterroblastulae. This is, for example, clearly illustrated by the nemerteans, platyhelminths [coeloblastulae are described for proseriates, and acoels (Convoluta sp.) may also possess a blastocoel; J. A. M. van den Biggelaar, pers. comm., but they are lacking in polyclads], molluscs, annelids, tardigrades, sipunculans, and cnidarians (Uchida \& Yamada, 1968; Anderson, 1973; Verdonk \& Biggelaar, 1983; Thomas, 1986; Fautin et al., 1989; Galleni \& Gremigni, 1989; Rice, 1989; Eibye-Jacobsen, 1996/1997; Ellis \& Fausto-Sterling, 1997; Henry \& Martindale, 1997; Younossi-Hartenstein \& Hartenstein, 2000). This intraphylum character variation indicates the likelihood of widespread convergent evolution.

It it important to note that the differences between a typical coeloblastula and sterroblastula are not sharp. The only consistent difference that distinguishes solid from hollow blastulae is the amount of interstitial space between the basal surfaces of the blastomeres. It only takes a tiny amount of intercellular fluid to accumulate between the basal sides of the blastomeres, e.g., nematodes (Schierenberg, 1997), to change from a solid sterroblastula to a hollow coeloblastula, and it is easy to discern a continuum in organization from a solid sterroblastula to a coeloblastula with only a minimal blastocoel, such as in gastrotrichs, nematodes and chaetognaths, to a 'textbook' coeloblastula as is found in echinoderms and cephalochordates. Even when the many '?'s in the data matrix of Zravy et al. (1998) are filled in, in view of these considerations it is doubtful whether this character will yield an unambiguous phylogenetic signal.

\section{Larval characters}

\section{Prototroch}

The prototroch is one of the defining features of a trochophore larva (Rouse, 1999; Nielsen, 2001). A prototroch is a pre-oral horseshoe or ring of (usually) compound cilia on multiciliate cells derived from trochoblasts, which exhibit a constant cell lineage and pattern of organization (Damen \& Dictus, 1994; Nielsen, 1995; Rouse, 1999). The prototroch 
cilia usually beat posteriorly, and together with the post-oral metatroch and a circumoral ciliated field it forms a complex character', the downstream-collecting system, which is discussed elsewhere in this paper.

\section{Character coding}

NI61: larva with prototroch $\mathrm{a} / \mathrm{p}$

P48; E40; R130; ZII42: prototroch a/p

Table 12. Scoring conflicts for prototroch.

\begin{tabular}{llll}
\hline & Absent & Present & ? \\
\hline Platyhelminthes & E40; P48; & NI61 & \\
& R130, ZII42 & & \\
Rotifera & R130 & P48; NI61 & ZII42 \\
Ectoprocta & & P48, ZII42 & NI61 \\
Annelida & E40 & P48; NI61; & \\
& (Clitellata) & R130, ZII42 & \\
& & (Polychaeta) & \\
Nemertea & E40; R130, & P48; NI61 & \\
& ZII42 & & R130 \\
Pogonophora & & E40 \\
\hline
\end{tabular}

\section{Character scoring and phylogenetic significance}

The polarized opinions about the homology of the ciliated larvae of platyhelminths and coelomate protostomes (trochophore larvae) have at least been in place since the mid 1980s. At this time Ehlers (1985) established his hugely influential and comprehensive phylogenetic system of the Platyhelminthes that provided the first effective ingredients for an argument against a line of reasoning with a long history that defended a phylogenetic link between the ciliated, and presumably planktotrophic, platyhelminth larvae and trochophore larvae (for different styles of this reasoning through time see Balfour, 1880; Jägersten, 1972; Ruppert, 1978; Salvini-Plawen, 1980b; Lacalli, 1984; Nielsen, 1995, 2001; Peterson et al., 2000a; Telford, 2001). Ehlers' platyhelminth phylogeny established a rigorous framework for interpreting the data on ciliated platyhelminth larvae that allowed the formulation of two key revisions.

First, only polyclad Götte's and Müller's larvae posses a pre-oral band of relatively long cilia (not compound) on multiciliate cells that rim the cili- ated lobes of the larvae that might be termed a prototroch. However, the exact cell lineage origin of this ciliated band has not been elucidated. Importantly, polyclads were not placed basal in Ehlers' phylogeny. Catenulida, Acoelomorpha, and Macrostomida are the earliest diverging taxa, and all are characterized by the lack of a biphasic life cycle with a ciliated larva (the significance of the Luther's larva of the catenulid Rhynchoscolex simplex with multiple pre-oral bands is uncertain at best). Consequently, the likelihood of a ciliated larval form in the ground pattern of the Platyhelminthes would be low. This conclusion can essentially be maintained, despite changing positions of polyclads within the Platyhelminthes in more recent cladistic analyses (see Littlewood et al., 1999a), and the removal of the acoelomorphs from the Platyhelminthes on the basis of molecular data (Ruiz-Trillo et al., 1999, 2002; Jondelius et al., 2002; Telford et al., in press).

Second, the restricted distribution of these ciliated larvae to certain taxa within the Polycladida even leaves the polyclad ground pattern uncertain (see discussion under Larva with strongly reduced hyposphere for references). Therefore, it can be concluded that evidence for the existence of a prototroch in the ground pattern of the platyhelminths (NI61) is very weak. This argument based on information about the phylogenetic distribution of larval characters within phyla is also followed by others such as Ax (1995) and Haszprunar et al. (1995). Arguments in favor of the primitive status of polyclad larvae result either from equating morphological similarity with homology, or from a priori assuming that convergent evolution is highly unlikely.

A closely parallel argument can explain the disagreement about the presence of a prototroch in Nemertea. Ciliated pilidium larvae possessing a ciliated band are restricted to the heteronemerteans and the palaeonemertean Hubrechtella dubia (Henry \& Martindale, 1997). The lack of a cladistic hypothesis of nemertean relationships hindered a definitive conclusion until very recently. However, the molecular phylogenetic analysis of Thollesson \& Norenburg (2003) based upon four different genes (nuclear 28S rRNA, H3, and mitochondrial 16S rRNA and COI) strongly suggest that pilidium lar- 
vae evolved once within the Nemertea as a synapomorphy of Heteronemertea $+H$. dubia. An earlier more restricted molecular phylogeny of the Nemertea based upon 18S rDNA sequences (Sundberg et al., 2001 ) is consistent with this conclusion. Interestingly, Maslakova et al. (1999) and Maslakova \& Norenburg (2001) reported purported intracapsular development of a "hidden larvae" in the hoplonemertean Tetrastemma candidum, and hints of a prototroch in the directly developing palaeonemertean Carinoma tremaphoros, respectively. These findings might suggest the possibility that indirect development may be ancestral for the entire phylum Nemertea. However, the significance of these findings remains uncertain.

Nevertheless, Nielsen $(1995,2001)$ (followed by Peterson \& Eernisse, 2001) interprets the classical cell lineage analyses of Hörstadius (1937) and the later cell lineage studies of Henry \& Martindale (1998a) as providing support for the thesis that the large ciliary band of pilidium larvae is homologous to a prototroch and thus part of the nemertean ground pattern. Henry \& Martindale (1998a) found that in the pilidium larva of Cerebratulus lacteus the cells corresponding to the prototroch-forming cells in other phyla at least also contribute to the prototroch, but in addition other cells contribute to the pilidium ciliary band as well. Gene expression experiments of a trochoblast specific gene construct (derived from molluscs) in nemerteans (Biggelaar et al., 1997; Loon \& Biggelaar, 1998) does not support the existence of a prototroch in the pilidium of Cerebratulus (Klerkx, 2001, and pers. comm.).

Nielsen $(1987,1995,2001)$ advocates the homology of the rotiferan trochus with the prototroch and the cingulum with the metatroch of trochophore larvae, an argument followed by Peterson \& Eernisse (2001). Note that P48 accidentally misscored Rotifera in the data matrix. Although these homology proposals are not buttressed by cell lineage data on the source of the ciliary bands in rotifers, they are in accordance with the widespread view that rotifers are paedomorphic animals with respect to taxa that possess trochophore larvae (see Jenner, 2001b for logical conflict between this hypothesis and phylogenetic evidence in Nielsen, 2001). However, available comprehensive morphological or total evidence $(+18 \mathrm{~S}$ rDNA sequence data) cladistic analyses frequently position Rotifera outside a clade Trochozoa (characterized by trochophore larvae) (Meglitsch \& Schram, 1991; Nielsen, 1995; Nielsen et al., 1996; Zrzavý et al., 1998, Zrzavý, 2003; Sørensen et al., 2000; Peterson \& Eernisse, 2001). The paedomorphic origin of Rotifera and homology of their ciliary bands with those of trochophore larvae thus remain dependent upon out-group comparison and the assumption of a trochus and cingulum in the rotiferan ground pattern.

Nielsen $(1995,2000,2001,2002 a)$ suggest that the corona of gymnolaemate ectoproct cyphonautes larvae may be homologous to a prototroch. This is based on the labeling of blastomeres according to the nomenclature of spiral cleavage, with a resulting identification of the source of the corona as being very similar to that found for the prototroch in spiralians. However, although the corona may be composed of multiciliate cells that normally beat in the same direction as prototroch cells, the coronal cilia are not compound as is typical for prototrochs. In addition, the corona partakes in a unique feeding mode distinct from that of the downstream feeding system (see discussion below) of which prototrochs are a part (Nielsen \& Riisgård, 1998; Nielsen, 2002b). In view of the continuing uncertainty about ectoproct phylogeny (Woollacott \& Harrison, 1997; Todd, 2000) and the concomitant uncertainty about the ancestral ectoproct life cycle, the scoring of the prototroch as a '?' seems the best option (NI61).

The presence of a prototroch in clitellates is primarily based on the presence of trochoblasts and transient ciliation in the embryos of some species (Rouse, 1999). The absence of a genuine prototroch is likely secondary, an argument that is indirectly supported by the undoubted nesting of the clitellates within a trochozoan clade, and possibly within a paraphyletic Polychaeta (Westheide et al., 1999; McHugh, 2000; Martin, 2001). Polychaetes are typically considered to be more representative of the annelid ground pattern than the clitellates.

There is some disagreement about the presence of a prototroch in Pogonophora (including Vestimentifera). A pre-oral ciliary band of multiciliated cells has been demonstrated in the larvae and juveniles of pogonophorans and vestimentiferans (Gardiner \& Jones, 1994; Southward, 1999; Nielsen, 
2001), but the interpretation of this band as a prototroch is not accepted by all current workers (see Salvini-Plawen, 2000). However, it should be noted that the ciliary bands or rings may be present before a stomodeum or mouth develops, making the exact identification of these bands problematic.

The scoring of a prototroch in Cycliophora (P48) deserves a final comment. This unambiguous scoring belies the tentativeness of the clues offered by the morphology of the chordoid larva. Funch (1996; see also Kristensen \& Funch, 2002) interprets the chordoid larva as a "modified trochophore" chiefly on the basis of its ciliation pattern, with two anterior bands, a ventral field, and a ciliated foot. From anterior to posterior these bands are compared to the prototroch, metatroch, neurotroch, and telotroch of trochophore larvae. However, neither of the two key diagnostic features that allow one to designate a band of multiciliate cells as a prototroch is present. The cellular source of the ciliated bands is unknown, and the lack of a digestive system and mouth in the chordoid larva does not allow the confirmation of a pre-oral position of the anterior-most ciliary band or the post-oral position of the second band (presumed metatroch). Other types of pre-oral bands are known in annelid larvae, such as akrotrochs and meniscotrochs (Rouse, 1999), and additional information is therefore needed to ascertain the identity of the ciliary bands in the chordoid larva. The fact that the chordoid larva is not planktotrophic argues against the presence of a metatroch homologue. Metatrochs are typically lacking in lecithotrophic larvae (Strathmann, 1993; Nielsen, 2001). Also, the cilia of the presumed cycliophoran metatroch beat laterally (Funch, 1996: 236), which appears to indicate a direction of ciliary beat opposite to those of typical metatrochs, which beat towards the prototroch. Similarly, information on the direction of ciliary beat has created ambiguity about the interpretation of the post-oral ciliary band in sipunculan pelagosphera larvae, but I do not regard this data alone to be sufficient for rejecting the presence of a metatroch.

In conclusion, the different scorings of a prototroch for the platyhelminths, nemerteans, rotifers, ectoprocts, and clitellates can all be defended, given that one ignores uncertainties about ground pattern states. For the platyhelminths and the nemerteans, the availability of a phylogenetic framework allows a relatively strong case to be built for the lack of a prototroch in the ground pattern. Not until the ground pattern states of the other phyla become better known, will we be able to decide whether the prototroch evolved once and reversed subsequently in various phyla such as Gnathostomulida (Nielsen, 2001; Peterson \& Eernisse, 2001) or evolved convergently in different phyla.

\section{Metatroch}

A metatroch is a post-oral ciliary band of compound cilia on multiciliate cells found in protostome larvae. The direction of ciliary beat typically is opposite that of the prototroch, i.e., directed anteriorly.

\section{Character coding}

P49; R131; ZII43: metatroch a/p

Table 13. Scoring conflicts of metatroch.

\begin{tabular}{llll}
\hline & Absent & Present & $?$ \\
\hline Rotifera & R131 & $\begin{array}{l}\text { P49; ZII43 } \\
\text { (Bdelloida, } \\
\text { Monogononta }\end{array}$ & P49 \\
Mollusca & R131 & ZII43 \\
\hline
\end{tabular}

\section{Character scoring and phylogenetic significance}

Nielsen $(1987,1995,2001)$ assumes the homology of the rotiferan cingulum and the metatroch of trochophore larvae, followed by Peterson \& Eernisse (2001) and Zrzavý (2003). It should be noted that the scoring for a prototroch (ZII42) and metatroch (ZII43) in the Bdelloida and Monogononta is in conflict in Zrzavý (2003). Although Zrzavý argues that he provisionally accepts the homology of the ciliary bands in the rotifers and trochozoans, the bdelloid and monogonont rotifers are nevertheless scored as possessing a metatroch but as "?" for a prototroch. Further conflict is introduced into the data matrix of Zrzavý for ZII47, which codes for an opposed-band larval feeding system. See under Prototroch and Downstream-collecting ciliary system for further discussion.

A metatroch is found in the bivalves and gastro- 
pods among molluscs, making it unlikely that a metatroch is part of the molluscan ground pattern. The character state found in the nearest out-groups then becomes relevant. Although recent cladistic analyses have not yet come to an agreement on the most likely sister group of the molluscs, most of them posses a metatroch. However, the detailed analysis of Rouse (1999) indicates that a metatroch has evolved independently multiple times within Polychaeta (optimization criterion independent).

P49 score Cycliophora for having a metatroch. See discussion under Prototroch for arguments against this scoring.

Nielsen $(1987,2001)$ argues against the scoring for R131 and P49 that the post-oral band of compound cilia in sipunculan pelagosphera larvae is not a metatroch but instead is an additional ciliary band that evolved within Sipuncula. Nielsen's chief argument is that the ciliary beat of this band is directed posteriorly, a direction that is opposite that of metatrochs in other phyla. However, an emphasis on positional information allows the identification of this post-oral ciliary band as a metatroch.

\section{Larvae or adults with downstream-collecting cili- ary system}

The most widely adopted definition of a downstream collecting ciliary system is the presence of two opposed ciliary bands composed of multiciliate cells that are used in feeding (Nielsen, 1987; 1995; Nielsen \& Riisgård, 1998). This system is also commonly referred to as the opposed band or double band system (Strathmann, 1978, 1993; Rouse, 2000a; Riisgård et al., 2000), and when strictly larvae are considered, it refers to the presence of both a pre-oral prototroch of relatively long cilia and a post-oral metatroch of relatively short cilia that enclose a circumoral ciliated food groove (cells with single cilia). However, to accommodate several polychaete taxa with feeding larvae lacking a metatroch (solely using the prototroch), Rouse redefined a downstream-collecting ciliary system as any ciliary system that uses the prototroch for downstream feeding (Rouse, 2000b, c).

\section{Character coding}

N22; NI21; S29; Z136: larvae or adults with downstream-collecting ciliary system $\mathrm{a} / \mathrm{p}$

M46: downstream particle capture/upstream particle capture in adults

M47: downstream particle capture/upstream particle capture in larvae

E42: nutritive metatroch with opposed bands $a / p$ Ca 17: downstream (multiciliate)/upstream (monociliate) feeding system

R140; ZII47: opposed band larval feeding a/p

\section{Character scoring and phylogenetic significance}

The scoring of M46 and M47 is highly problematic, and therefore not included in table 14. These characters are discussed below. The phylogenetic significance of downstream-collecting ciliary bards has generated a considerable amount of discussion in the recent literature. Nielsen's $(1985,1987,1995$, 1998a, 2001) detailed studies are responsible for the introduction of downstream- and upstreamcollecting ciliary systems into recent phylogenetic

Table 14. Scoring conflicts of downstream-collecting ciliary system.

\begin{tabular}{|c|c|c|c|c|}
\hline & Absent & Present & $?$ & Proposed scoring \\
\hline Ectoprocta & N22; NI21; S29; Z136 & $\mathrm{Ca} 17$ & ZII47 & Absent \\
\hline Rotifera & $\mathrm{R} 140$ & $\begin{array}{l}\text { N22; NI21; S29; } \\
\text { Z136; ZII47 }\end{array}$ & & Present in adults or? \\
\hline Platyhelminthes & S29; E42; R140; ZII47 & N22; Z136 (only in Polycladida) & NI21 & Absent? \\
\hline Sipuncula & $\begin{array}{l}\text { N22; NI21; S29; Z136; } \\
\text { ZI147; R140; E42 }\end{array}$ & $\mathrm{Ca} 17$ & & Absent \\
\hline Mollusca & $\mathrm{R} 140$ & $\begin{array}{l}\text { N22; NI21; S29; Z136; E42 (only } \\
\text { polymorphic in Conchifera) }\end{array}$ & ZII47 & Absent \\
\hline Nemertea & $\mathrm{R} 140 ; \mathrm{E} 42 ; \mathrm{ZII} 47$ & N22; S29; Z136 & $\mathrm{N} 121$ & Absent \\
\hline Myzostomida & R140; ZII47 & $\mathrm{Z} 136$ & & $?$ \\
\hline
\end{tabular}


discussions. Nielsen initially developed his ideas of the phylogenetic significance of these distinct types of ciliary feeding systems in close connection with his controversial trochaea theory (Nielsen \& Nørrevang, 1985). Other authors have readily incorporated these features into their own phylogenetic analyses (Meglitsch \& Schram, 1991; Eernisse et al., 1992; Zrzavý et al., 1998; Rouse, 1999; Sørensen et al., 2000). In the contemporary phylogenetic literature, C. Nielsen and G. W. Rouse are the most conspicuous exponents of conflicting views on the evolutionary significance of downstream-collecting ciliary systems. Nielsen has vigorously defended the evolution of a larval downstream-collecting ciliary system in the stem lineage of Protostomia, and its retention as a plesiomorphy in the ground pattern of the Spiralia (Nielsen, 1985, 1987, 1995, 1998a, 2001). In contrast, Rouse (1999, $2000 \mathrm{a}, \mathrm{b}, \mathrm{c})$ explicitly tested Nielsen's hypothesis with a cladistic analysis, and reached a rather different conclusion that emphasized the multiple independent origins of larval downstream-collecting ciliary systems between different trochozoan phyla as well as within the polychaetes. However, there are problems with the testing power of Rouse's approach, principally in connection with uncritical selection of taxa and characters, and character scoring (Jenner, in press). Before I discuss the different views on the evolution of downstream feeding systems, I will first attempt to resolve the observed scoring conflicts.

The scoring of the ectoprocts for $\mathrm{Ca} 17$ and ZII47 is inaccurate, and results from an undue emphasis on the presence of multiciliate cells making up the ciliary bands rather than on the actual feeding mechanism in which these ciliary bands participate. Ectoproct larvae and adults have a unique morphology of the ciliary bands (Nielsen, 2002b), and they employ a unique type of upstream-collecting ciliary mechanism that uniquely uses multiciliate cells (Nielsen \& Riisgård, 1998).

The scoring of $\mathrm{Ca} 17$ is also incorrect for the sipunculans. Sipunculan trochophores are lecithotrophic, and when a planktotrophic pelagosphera larva is developed later in ontogeny they do not use opposed-band feeding. However, their exact feeding mechanism remains unknown to date.

Zrzavý (2003) scored the bdelloids and mono- gononts as "?" for a prototroch, and as possessing a metatroch. Yet, despite the fact that both a prototroch and a metatroch are essential components of a downstream system, bdelloids and monogononts are unambiguously scored as possessing an opposedband ciliary feeding system.

Rouse (1999) is the only study that included a character on downstream ciliary feeding that did not score rotifers as present. This has two reasons. First, R140 only coded for the presence of a downstream ciliary system in larvae, and rotifers are considered as lacking larvae. Second, although the rotiferan ground pattern remains uncertain in the absence of a generally accepted cladistic hypothesis of their internal relationships, most recent authors accept that a ciliary system with opposed bands (pre-oral trochus and post-oral cingulum) is derived, albeit commonly present, within the phylum (Haszprunar et al., 1995; Lorenzen, 1996c). The uncertainty about the rotiferan ground pattern has allowed the other analyses to adopt an opposite scoring. Interestingly, out-group comparisons in the most comprehensive and most recent morphological and total evidence analyses do not support the plesiomorphy of a downstream-collecting ciliary system in rotifers (Nielsen et al., 1996; Nielsen, 2001; Zrzavý et al., 1998, 2001; Peterson \& Eernisse, 2001). Finally, it should be noted that the scoring of a downstream-collecting ciliary system in seisonid rotifers (S29) is erroneous. Seisonids merely possess some rudimentary circumoral ciliation (Markevich, 1993; Ahlrichs, 1995).

Nielsen (2001: NI21) reconsidered the scoring of a downstream ciliary system for platyhelminths (N22), and changed it from present to '?' based on the presence of presumed planktotrophic polyclad larvae (see Z136). Considering that downstream ciliary feeding using compound cilia has neither been observed for polyclad larvae (Nielsen, 1987, 2001), nor for any other platyhelminth, larval or adult, the scoring of S29, E42 and R140 is the most appropriate (absence of downstream ciliary system).

Within the molluscs, downstream ciliary feeding is only known from gastropods and bivalves, while the larvae of the other major taxa are strictly lecithotrophic. Available evidence from comparative embryology and gastropod phylogenetics indicates the likelihood of a primitive lecithotrophic 
gastropod larva, while the primitive larval type of bivalves remains uncertain (Haszprunar, 1992; Haszprunar et al., 1995; Cragg, 1996; Ponder \& Lindberg, 1997; but see Page, 1994). This makes the presence of a lecithotrophic larva in the ground pattern of the Mollusca currently the most likely hypothesis, in agreement with the scoring of R140.

Direct observations on the feeding mechanism of presumed planktotrophic nemertean pilidium larvae are lacking (Nielsen, 1987, 1998a), justifying the scoring of a '?' (NI21). Moreover, because pilidium larvae are not assumed to be part of the nemertean ground pattern, the scoring of N22, S29 and Z136 is inappropriate, and the scoring of absence of downstream ciliary feeding (E42, R140) is currently the most defensible option.

Rouse (1999, 2000b) scored the myzostomids as not having feeding larvae although both a prototroch and metatroch may be present, while Zrzavý et al. (1998) scored them for having downstream ciliary feeding. The scoring for myzostomids is currently uncertain at best. The feeding mechanism of myzostomid larvae remains unknown (Jägersten, 1972; Nielsen, 1998a), but Jägersten (1972) noted that myzostomid eggs are relatively yolk-poor, and that larvae possess well-differentiated guts with a mouth and anus, which is often indicative of larval planktotrophy. It should also be noted that pictures of the trochophores of Myzostomida in the recent literature sometimes label the presence of a prototroch, but these illustrations clearly show a postoral ciliary band that cannot be interpreted as a prototroch (see for example Grygier, 2000: fig. 2.8; Nielsen, 2001: fig. 19.1; Jägersten, 1972: fig. 43; all these illustrations are based on Jägersten, 1939). Nevertheless, young trochophore larvae of Myzostomum parasiticum may possess two bands, presumably a pre-oral prototroch and a post-oral metatroch (Prenant, 1959: fig. 571), but between three and four days of age the (meta-)trochophore appears to lose its pre-oral ciliary band (compare figs. 571D and E in Prenant, 1959).

The scoring for Cycliophora also deserves a brief discussion, even though the available analyses do not exhibit scoring conflicts. The cladistic analyses that included this taxon (Sørensen et al, 2000; Zrzavý et al., 1998) unambiguously score the presence of downstream-collecting ciliary feeding, while
Z135 also scores larval planktotrophy for Cycliophora. First, none of the free-living larval stages of Symbion pandora has a digestive tract, ruling out larval planktotrophy, and necessitating a rescoring of Z135. Second, the homology of the downstream-collecting ciliary system of the adult feeding stage of $S$. pandora to other downstream ciliary systems is not straightforward. In their detailed functional study of downstream-collecting ciliary systems Riisgård et al. (2000) describe for the first time the feeding mechanics of the cycliophoran buccal funnel. After defining the downstream system as being composed of two parallel ciliary bands of compound cilia surrounding a ciliated mouth field of cells with separate cilia, they conclude that the ciliary feeding system of $S$. pandora conforms to this definition, and it is similar in structure and function to the downstream ciliary systems observed in the other taxa. However, the morphology of the cycliophoran ciliary system does not conform precisely to the adopted definition. The feeding stage of $S$. pandora possesses a single ring of compound cilia, not two bands (Riisgård et al., 2000 interpret the opposite sides of the single circle of cilia as "opposed bands"), and a circumoral ciliated field of cells with separate cilia is lacking (the ciliation of the mouth cavity consists of several rows of compound cilia on multiciliate cells). Thus, although the cycliophoran ciliary feeding system is functionally very similar to that of spiralian ciliary feeding trochophore larvae, its detailed morphology creates uncertainty about its homology with other downstream systems, in contrast to the scoring of S29. Funch \& Kristensen (1997: 469) similarly conclude that "the cilia of the mouth ring and buccal funnel in the Cycliophora do not work with opposed bands."

Several comments on character coding are in order. It should be noted that the character states of M46 and M47 do not display a complement relation, resulting in misscoring of all phyla that lack ciliary feeding systems as adults or larvae. This resulted in the scoring of downstream-collecting (and upstream-collecting) ciliary systems in phyla such as mesozoans and nematodes. Obviously, M46 and M47 have to be recoded.

The scoring of E39 will not be discussed here because the definition is not restricted to larval 
feeding, but also refers to larval locomotion, resulting in a broader range of scored taxa. The definition of an opposed-band ciliary feeding system for E42 reads "nutritive metatroch with opposed bands; postoral (segmentally added) paired ciliary bands beating in opposite directions and serving in food capture," and is claimed to be derived from two sources in the literature, viz., Strathmann (1978) and Salvini-Plawen (1988). However, these sources do not offer such a definition of an opposed-band feeding system. Instead, the definition incorporates one pre-oral and one post-oral ciliary band that beat towards each other. Multiple post-oral ciliary bands are never involved in this feeding mechanism.

Quite different phylogenetic significances have been attached to this character in recent phylogenetic studies of the Metazoa (see also table 3 in Jenner \& Schram, 1999). Nielsen has been the strongest advocate of a single evolutionary origin of larval downstream ciliary feeding, but Nielsen's computerassisted parsimony analyses (Nielsen et al., 1996; Nielsen, 2001) have not confirmed his narrativebased scenario. N22 was found to be apomorphic for Spiralia + Ectoprocta, and convergently evolving in rotifers, with several reversals in the sipunculans, ectoprocts, and panarthropods. NI21 originated twice independently, once in molluscs + annelids, and once in the rotifers + entoprocts, with reversals in the gnathostomulids and panarthropods. The convergent origin of downstreamcollecting ciliary feeding is also supported by the analyses of Zrzavý et al. (1998) (three unambiguous convergent origins of Z136 under ACCTRAN in rotifers, polyclads and the rest), and Rouse (1999) (multiple convergences of R140 in entoprocts, echiurans, and various times within the polychaetes). In fact, the analysis of Sørensen et al. (2000) provided the only recent cladistic support for a single origin of downstream ciliary feeding. However, the single origin of S29 is caused by the scoring of 'inapplicability' or '?' in taxa such as Gnathostomulida and Micrognathozoa, instead of 'absence' of downstream ciliary feeding. Inapplicability scoring for, for example, Gnathostomulida can be defended if S29 strictly coded larval morphology (gnathostomulids lack larvae). However, S29 also coded for the adult situation, and therefore logic would dictate the scoring for the gnathostomulids as lacking a downstream ciliary system, just as the rotifers (adults) were unambiguously scored as possessing it. This would create results in accordance with the convergent evolution of downstream ciliary feeding as suggested by the other recent morphological cladistic analyses. Alternatively, S29 is only applicable for taxa with multiciliate cells, and therefore inapplicable for the gnathostomulids. However, Micrognathozoa do have multiciliate cells and should therefore be scored as lacking a downstream system.

The major obstacle for reaching agreement on the phylogenetic significance of downstream ciliary feeding (apart from estimates of the relative likelihood of character gains and losses) is uncertainty and/or disagreement about the ground patterns of several phyla. For example, opposed band larval feeding in the annelids is only known (functional studies) for three families: Serpulidae, Oweniidae (Miner et al., 1999), and Capitellidae (Pernet \& Schroeder, 1999), while several other families do possess the necessary ciliary bands but confirmation of their function is lacking. Yet, with the exception of Rouse's (1999, 2000a, b, c) analyses, the annelids, or at least the polychaetes are scored as unequivocally having downstream-larval ciliary feeding. And even when character mapping on a phylogeny suggests a particular scenario of character evolution, some authors maintain that reliable inferences have to await a better understanding of the relative probabilities of character change in different directions (Strathmann \& Eernisse, 1994; Budd \& Jensen, 2000). Disagreements divide researchers such as Nielsen and Rouse about the relative likelihood of independent gains or losses of larval downstream feeding.

If we accept parsimony optimization of characters on a phylogeny, then current analyses at least suggest a certain degree of convergence of downstream ciliary feeding in both adults and larvae, in particular supporting the independent origin of this trait in the rotifers and the other taxa.

Larva with strongly reduced hyposphere

This character refers to larvae in which the postprototrochal region (hyposphere) is significantly reduced in size. However, because it is specifically 
applied to platyhelminth and nemertean larvae in which the existence of a prototroch (defined on the basis of the cell lineage of the prototrochal cells) is contentious, the hyposphere can be regarded as the part of the larva posterior to the rim of long cilia in polyclad and pilidium larvae. The infusion of a phylogenetic assumption into the definition of this feature makes it a particularly interesting character.

\section{Character coding}

S28; NI20: larva with strongly reduced hyposphere $\mathrm{a} / \mathrm{p}$

\section{Character scoring and phylogenetic significance}

The reduction of the larval hyposphere is interpreted as a synapomorphy for Platyhelminthes and Nemertea (Parenchymia) in Nielsen (1995), Nielsen (2001), and Sørensen et al. (2000). Support for the validity of this character is largely derived from the detailed comparative studies of larval nervous systems and ciliary bands performed by Lacalli (1982, 1983, 1984; Lacalli \& West, 1985). These studies demonstrated various morphological similarities shared between the polychaete trochophore larvae, the polyclad Müller's and Götte's larvae (lobophore larvae), and the nemertean pilidium larvae. However, the definition of this character mixes a justifiable morphological component with a much more contentious assumption.

The character refers explicitly to a larva with a reduced hyposphere, which embodies an a priori assumption of the direction of evolutionary change: the derivation of a reduced hyposphere from an ancestral state with a more developed hyposphere. Sørensen et al. (2000: 301) qualify the character as "..a trochophora type of larva with a strongly reduced hyposphere," while Nielsen (1995: 214) concludes that "...the larvae could perhaps be interpreted as trochophores with strongly reduced hypospheres..." These interpretations appear to be quite colored by the trochaea theory according to which "...these larvae should be modified trochophores..." Nielsen (1995: 211). The introduction of a phylogeny-dependent element into a cladistic character is unjustifiably circular, and is in direct conflict with the main goal of cladistic analyses, namely the establishment of patterns of character distribution that may function as starting points of hypotheses of evolutionary character transformation. At the very least, in order for this phylogeny-dependent character to be valid, parenchymians should be nested within a clade of protostomes that are unambiguously characterized by the possession of trochophore larvae with a more fully developed hyposphere. Although this may be consistent with the results from Nielsen et al. (1996) and Sørensen et al. (2000), other recent studies place the platyhelminths and nemerteans outside a clade characterized by trochophore larvae, or do not indicate any close relationship between these two phyla in the first place (Eernisse et al., 1992; Haszprunar, 1996a; Rouse \& Fauchald, 1995; Giribet et al., 2000).

Since this character is chiefly based upon information from the studies of Lacalli, it should be noted that Lacalli himself is very reserved in drawing concrete evolutionary conclusions with regard to the similarities of polyclad lobophore and pilidium larvae (Lacalli, 1982; Lacalli \& West, 1985). Interestingly, Lacalli's only explicit evolutionary scenario exhibits an opposite polarity of change to that proposed by Nielsen (1995) and Sørensen et al. (2000). Lacalli (1984) envisions that polyclad larvae are the more ancestral larval form from which trochophore larvae could have evolved.

Although the typical helmet-shaped pilidium indeed appears to possess a very small post-trochal area or hyposphere, various different types of pilidium larvae are known, including two types called Pilidium recurvatum and $P$. incurvatum (figs. 21 and 23 in Jägersten, 1972; fig. 27.1 in Nielsen, 1995). These two types possess a hyposphere of "normal" proportions, comparable in relative dimensions to that of many trochophore larvae, and including a posterior telotroch. Interestingly, both Jägersten (1972: 99) and Nielsen (1985: 257) suggested that the typical helmet-shaped pilidium larva may well have evolved from this type of pilidium with a normally proportioned hyposphere. Also, different types of Müller's and Götte's larvae may differ in the extent of their hyposphere, so that some forms may possess quite sizable hypospheres. The determination of the primitive form of either the pilidium or 
the Müller's and Götte's larvae remains pure conjecture at this time. These considerations further lessen the probability that a small or reduced hyposphere is homologous in polyclad and pilidium larvae.

Distinct from the difficulties that are introduced through the blending of a proper morphological component and an unnecessary a priori assumption of the direction of evolutionary change, it is questionable whether the similarities of polyclad and nemertean pilidium larvae could be scored as primary homologies in the first place. First, the placement of the polyclads within the platyhelminths on the basis of current morphological and molecular phylogenetic data does not support the hypothesis that polyclad Müller's and Götte's larvae are part of the platyhelminth ground pattern (Carranza et al., 1997; Campos et al., 1998; Zrzavý et al., 1998; RuizTrillo et al., 1999; Giribet et al., 2000). Of course, this does not prove that these polyclad larval types could not in principle be primitive platyhelminth larvae retained from the ancestral life cycle, but current data do not even unambiguously support the presence of Müller's and Götte's larvae in the polyclad ground pattern. More likely, these larvae evolved within the polyclads (Ehlers, 1985; Ax, 1995). Second, new phylogenetic evidence supports the notion that pilidium larvae are not present in the nemertean ground pattern (Thollesson \& Norenburg, 2003). Earlier, Nielsen $(1995,2001)$ himself admitted as much. He states that (2001: 288): “...the life cycle involving a pilidium larva is definitely not ancestral." This statement implies that pilidium larvae have evolved within a monophyletic Nemertea, and that they are therefore inconsequential for linking Nemertea to any other phylum (see Jenner, $2001 \mathrm{~b}$ for an example of logical conflict in Nielsen, 2001 centered upon the presumed primitiveness of pilidium larvae).

\section{Prototrochal lobes}

This character describes whether or not the prototroch is located on lobes.

\section{Character coding}

M66: prototrochal lobes (velum, pilum) a/p Z139: prototroch developed as ciliated lobes $a / p$

\section{Character scoring and phylogenetic significance}

M66 and Z139 both score only Nemertea and Mollusca. The homology of this feature between nemerteans and mollusks is unlikely. The presence of a pilidium larva in the nemertean ground pattern is contradicted by new phylogenetic evidence (Thollesson \& Norenburg, 2003). Molluscan veliger larvae are only found within bivalves and gastropods. Even assuming that they are part of the bivalve and gastropod ground patterns, they are most likely not present in the ground pattern of the entire phylum (lacking in the aplacophorans, polyplacophorans, monoplacophorans, scaphopods, and cephalopods) (Nielsen, 1995, 2001). [However, see Cragg, 1996, Salvini-Plawen \& Steiner, 1996, Hadfield et al., 1997, and Ponder \& Lindberg, 1997 for arguments based upon the distribution of veligers within bivalves and gastropods that they may not be ancestral characters for both taxa, and that convergent evolution of the veliger even within the gastropods is likely]. This character only illustrates the prevalence of convergent evolution, and will only introduce noise into the phylogenetic analyses of Meglitsch \& Schram (1991) and Zrzavý et al. (1998).

\section{Body cavities}

"Hohlräume sind begrifflich schwer zu fassen, da sie im Prinzip keine Eigenstruktur aufweisen, so dass sie nur indirekt begriffen werden können. Ein Vergleich von Hohlräumen stellt sich deshalb als besonders schwierig dar." -Ahlrichs (1995: 151)-

"In der Tat lassen sich Hohlräume - also sozusagen negative Strukturen - nicht ebenso homologisieren wie Organe mit ihren positiven Strukturen"-Remane (1963a: 483-384)-

This section discusses body cavities located roughly between the epidermis and gut. The interpretation of metazoan body cavities can be regarded as one of the most controversial, yet influential, subjects of comparative zoology perhaps only being rivaled in scope and intensity by discussions on the evolutionary significance of larval forms. The evolutionary importance that body cavities have held in 
past thinking is reflected by extensive discussions of various competing historical theories of animal evolution. These centered on divergent interpretations of body cavities such as the enterocoel and gonocoel theories, and by the central role of body cavities in the naming of many different higher level animal taxa (Coelomata, Pseudocoelomata, Rhynchocoela [nemerteans], Paracoelomata [approx. Ecdysozoa sensu lato Zrzavý et al., 1998], Procoelomata [stem-group metazoans above platyhelminth grade of organization; Bergström, 1989], Archicoelomata [stem-group bilaterians and some modern descendants [Ulrich, 1972], Coelomopora [Hemichordata + Echinodermata]).

Because a cavity by its nature lacks defining features, body cavities can only be characterized with reference to surrounding structures, such as a peritoneal coelomic lining, or structures internal to the body cavity, e.g., coelomocytes. Since the nature of an animal's body cavity is intimately related to many of these structures, attempts to understand the evolution of body cavities has to address a considerable variety of topics, including nephridial, circulatory, muscular, and gonadal systems, body segmentation, functional morphology of locomotion, and life cycles stages.

Acoeloms, pseudocoeloms, and coeloms, primary and secondary body cavities

According to traditional concepts of body cavity design, bilaterian metazoans can be arranged into three grades of organization: acoelomate, pseudocoelomate, and coelomate (Hyman, 1940; 1951a; Ax, 1995; Rieger, 1996a). This structural division is frequently interpreted as a phylogenetic series in the classical Anglo-Saxon literature, symbolizing the traditional textbook tree that is supposedly rooted in Hyman's influential multivolume work on The Invertebrates. However, a close reading of Hyman reveals that although she regarded the structural distinctions to be established on firm anatomical observations, the phylogenetic significance of body cavities was less clear (Jenner, 2000). This threefold distinction is based primarily on light microscopical data, but since then, electron microscopical studies have necessitated a revision of these concepts (Ruppert, 1991a; Rieger, 1996a) prima- rily by showing that acoelomate and pseudocoelomate organizations are arbitrary points along an ultrastructural continuum. In addition, insufficient attention has been paid to the nature of animal life cycles in the scoring of body cavity characters in recent cladistic studies, and this has seriously constrained recent thinking about the evolution of animal body cavities (see Jenner, 2001a).

Ultrastructurally there is a clear distinction between the acoelomate/pseudocoelomate organization on the one hand and the coelomate organization on the other. Acoelomate and pseudocoelomate animals can be said to possess a primary body cavity, which may be a retained blastocoel, and coelomate animals a secondary body cavity. A primary body cavity ranges from a virtual absence of any "cavity", such as the very narrow interstitial spaces in gnathostomulids, to a more spacious cavity lined by ECM and bordered by the basal surfaces of the epidermis and gut, such as found in priapulids. A secondary body cavity is always in contact with the apical surfaces of its mesodermal epithelial cell lining. These terms have been in the literature for a long time (Hyman, 1951a; Remane, 1963a; SalviniPlawen \& Splechtna, 1979), but they have not always been defined in a rigorous fashion, and in some cases different uses of these terms have been misleading. This paper will only discuss the secondary body cavity or coelom.

\section{Secondary body cavity, coelom}

"There is nothing to indicate that the various coeloms are homologous, and this opinion is now shared by most authors" -Nielsen (2001: 86)-

"It is reasonable to consider that the coelomic cavity is an autapomorphy distinguishing triploblasts from diploblasts" -Dewel (2000: 43)-

"Only one major morphological character remains as an unambiguous defining trait of the Bilateria: the coelom" -Martindale et al. (2002)-

How do we define a coelom? The scope of the chosen definition depends crucially on the criteria of homology that one employs. It is important to distinguish between different levels of comparison, rang- 
ing from descriptive histology and gross anatomy, to functional morphology and morphogenetic processes (Remane, 1963a; Siewing, 1969; SalviniPlawen \& Splechtna, 1979; Ahlrichs, 1995; Minelli, 1995). A failure to make this distinction has led to confusing controversies, especially in regards to particular structures in specific taxa, such as the nature of the molluscan gono-pericardial system (Salvini-Plawen, 1968; Salvini-Plawen \& Splechtna, 1979; Salvini-Plawen \& Bartolomaeus, 1995; Morse \& Reynolds, 1996), the nemertean rhynchocoel and circulatory system (Turbeville \& Ruppert, 1985; Turbeville et al., 1992; Bartolomaeus, 1993a; Ax, 1995; Nielsen, 1995), and the body coeloms of chaetognaths (Telford \& Holland, 1993; Christoffersen \& Araújo-de-Almeida, 1994; Schram \& Ellis, 1994; Shinn, 1994; Ghirardelli, 1995; Nielsen, 1995; Kapp, 1996; Stocker, 1998). The following section will discuss coeloms from three different perspectives: 1) structure, 2) ontogenetic origin, and 3) morphogenesis.

\section{1) Histological (ultrastructural), morphological, $\underline{\text { structural definition }}$}

To avoid unwarranted hypotheses of homology implied by the ambiguously used term 'coelom'. for mesothelium lined cavities in a wide variety of animals, Salvini-Plawen \& Bartolomaeus (1995) recommended the application of the term 'mesothelocoel' to the broad category of strictly structurally defined coeloms. This applies to all coelomic cavities irrespective of ontogenetic origin, structural elaboration, e.g., lined by a peritoneum or not, exact configuration, anatomical position, and function. Finding it unnecessary to introduce a new term, but recognizing the merit of a clear homology proposal, I will use in this paper the simple term "coelom' as equivalent to 'mesothelocoel.'

Since its original formulation by Haeckel in the late $19^{\text {th }}$ century, the definition of a coelom has been constrained with respect to the vertebrate pleuroperitoneal cavity with its peritoneal lining (Ruppert, 1991a). Hyman's often adopted definition reflects this constraint: "A true coelom is thus defined as a space between digestive tract and body wall which is bounded on all sides by tissue of entomesodermal origin and lined by peritoneum" (1951a: 23). This definition is echoed in many recent works, such as Brusca \& Brusca (1990), Willmer (1990), Meglitsch \& Schram (1991), Eernisse et al. (1992) (E17, 18), Brusca et al. (1997), Raff (1998), Stocker (1998), Anderson (1998), and Almeida \& Christoffersen (2000). It implies a pseudostratified or stratified coelomic lining in which muscle or epithelio-muscle cells are entirely separated from the coelomic fluid by a non-muscular epithelial peritoneum. The coeloms of various invertebrates reasonably conform to this definition. For example, chaetognath trunk and tail coeloms are at least partially covered by peritoneocytes (Shinn, 1994, 1997), polychaete perivisceral coeloms with peritoneocytes covering the somatic lining (Fransen, 1988; Bartolomaeus, 1994), the nemertean rhynchocoel with non-muscular lining cells (Turbeville \& Ruppert, 1985; Turbeville, 1991), and echinoderm podia lined with peritoneocytes (Rieger \& Lombardi, 1987). However, many other phyla do not answer this definition since their coeloms are lined by a simple myoepithelium lacking an overlying non-contractile peritoneum, e.g., many polychaetes (Fransen, 1988; Bartolomaeus, 1994), hemichordates (Benito \& Pardos, 1997), and some echinoderms (Rieger \& Lombardi, 1987). Current evidence suggests that peritoneal linings have evolved convergently within the coelomate bilaterians, such as within the polychaetes, sipunculans, echiurans, and echinoderms (Rieger, 1986a; Rieger \& Lombardi, 1987; Bartolomaeus, 1994). Note that Nielsen (1995, 2001) adopts a broad definition of a peritoneum that coincides with what I accept here as a definition of a mesothelium (Ruppert, 1991a), which designates both genuine non-contractile coelomic linings and myoepithelia as peritonea.

Other authors adopted a less restricted definition of a coelom, exemplified by Ruppert (1991a): a compartment in the connective tissue compartment, between epidermis and gastrodermis that is lined by a mesodermally derived epithelium, or mesothelium. Corresponding definitions are found in Nielsen (1985, 1995), and Haszprunar (1996a) (H26). For practical purposes, Ahlrich's (1995) purely topographical definition of a secondary body cavity as a compartment within a compartment, i.e., the primary body cavity, bounded by the gut and epidermis, corresponds closely to this definition of 
a coelom (note that Salvini-Plawen \& Bartolomaeus, 1995, strictly use this term to refer to a true 'body coelom'). Eernisse et al. (1992) coded at least ten characters (E14-23) directly related to body cavities, employing various homology criteria. Other recent phylogenetic studies either of the entire Metazoa, or a subset of taxa supplied no definition, but judging from their data matrices, Meglitsch \& Schram (1991) (M42), Zrzavý et al. (1998) (Z30), and Wallace et al. (1996) (Wa2) employed broad structural definitions of a coelom. Although gonocoels and metanephridia may also answer this broad definition of a coelom, they are not scored accordingly in these analyses.

\section{Character coding}

H26; Z30: histologically defined coelom a/p M42: no body cavity/coelom (metacoel)

\section{H28; Z32; ZI11; ZII19: (eu)coelom a/p}

M42 suggests the homology of the coeloms of protostomes with only the metacoel in deuterostomes, including lophophorates. Although this scoring is not explicitly outlined in the text, it in fact reflects an assumption of metazoan evolution that coincides with a theory propounded by SalviniPlawen $(1982,1985,1998$ a) on the origin of oligomerous metazoans (the lophophorates and deuterostomes).

Table 15. Scoring conflicts of histologically defined coelom (absent, present).

\begin{tabular}{llll}
\hline & Absent & Present & $\begin{array}{l}\text { Proposed } \\
\text { scoring }\end{array}$ \\
\hline Priapulida & $\mathrm{M} 42$ & $\mathrm{Z} 30$ & Absent \\
Chactognatha & $\mathrm{M} 42$ & $\mathrm{Z} 30$ & Present \\
Entoprocta & $\mathrm{Z} 30, \mathrm{H} 26$ & $\mathrm{M} 42$ & Absent \\
Myzostomida & $\mathrm{Z} 30, \mathrm{H} 26$ & $\mathrm{R} 125$ & Absent \\
Urochordata & $\mathrm{M} 42$ & $\mathrm{Z} 30$ & Present \\
\hline
\end{tabular}

\section{Character scoring and phylogenetic significance}

There have been different opinions about the nature of the body cavity of priapulids expressed in the literature (Land \& Nørrevang, 1985). However, it is probable that the priapulid ground pattern does
Table 16. Scoring conflicts of a eucoelom (absent, present).

\begin{tabular}{|c|c|c|c|c|}
\hline & Absent & Present & $?$ & $\begin{array}{l}\text { Proposed } \\
\text { scoring }\end{array}$ \\
\hline Mollusca & $\mathrm{H} 28 ; \mathrm{Z} 32$ & ZII19 & ZI11 & Absent \\
\hline Nemertea & $\mathrm{H} 28 ; \mathrm{Z} 32$ & ZII19 & $\mathrm{Z} 111$ & Absent \\
\hline Chaetognatha & & ZII19 & Z32; ZIII & Present \\
\hline Ectoprocta & $\mathrm{Z} 32$ & ZII19 & ZI11 & Present \\
\hline Arthropoda & $\mathrm{Z} 32$ & & ZI11; ZII19 & Absent \\
\hline Tardigrada & $\mathrm{Z} 32$ & & ZI11; Z1119 & Absent \\
\hline Onychophora & $\mathrm{Z} 32$ & & ZI11; ZII19 & Absent \\
\hline
\end{tabular}

not include a coelom lined by a mesothelium, contrary to Zrzavý et al. (1998). Instead, a spacious primary body cavity represents the likely priapulid ground pattern (Storch, 1991; Neuhaus, 1994; Ahlrichs, 1995; Schmidt-Rhaesa, 1996; Adrianov \& Malakhov, 1996). The body cavity is surrounded by non-epithelial muscle cells (Storch, 1991). Earlier reports of the presence of an epithelial (peritoneal) coelomic lining resulted from the incorrect interpretation of ECM (basal lamina) surrounding non-epithelial muscle cells as a coelomic epithelium (Land \& Nørrevang, 1985). Amebocytes may also be found in close apposition to the longitudinal body wall musculature (McLean, 1984; Adrianov \& Malakhov, 1996) that give the appearance of scattered coelomic lining cells. A genuine coelomic cavity that answers a histological definition occurs only in one, Meiopriapulus fijiensis, of the 18 currently described extant species of priapulids (Shirley \& Storch, 1999). The small coelomic cavities surrounding the mouth cone (in addition to the spacious non-coelomic major body cavity) of the meiobenthic priapulid Meiopriapulus fijiensis represent a peculiarity within priapulids (Storch, 1991). In addition, Meiopriapulus may be considered to be one of the most evolutionarily derived of the extant priapulids (Adrianov \& Malakhov, 1996; Wills, 1998).

Lack of robust ultrastructural data on the body cavity lining in the chaetognaths, the apparently aberrant developmental mechanism by which the body cavities arise in the embryo, and the questionable permanence of these cavities throughout ontogeny have provided room for debate on the nature of chaetognath body cavities and their phylogenetic placement (Hyman, 1959; Meglitsch \& Schram, 1991; Schram \& Ellis, 1994; Backeljau et 
al., 1993, Brusca \& Brusca, 1990; Willmer, 1990; Ghirardelli, 1995). Current ultrastructural information favors the interpretation that the chaetognath ground pattern comprises histologically defined coeloms in the head, trunk and tail regions of the adult body, in contrast to Meglitsch \& Schram (1991), e.g., Welsch \& Storch (1982); Shinn (1994, 1997); Kapp (1996). Nielsen (1985, 1995) considered the chaetognath coeloms to be autapomorphies, and he placed greater emphasis on the supposed aschelminth features of the chaetognaths. The suggestion by Telford \& Holland (1993) that the lack of a peritoneum suggests that the chaetognath coelom is a pseudocoelom either rests on the employment of the term peritoneum to refer to any mesothelium (see also Nielsen, 1995), or incorrectly interprets the peritoneum to be a diagnostic feature of a coelom, and it passes over the fact that at least an incomplete layer of peritoneocytes lines the adult trunk and tail coeloms (Welsch \& Storch, 1982; Shinn, 1994, 1997).

The scoring of a coelom in Entoprocta in Meglitsch \& Schram (1991) is a lapsus, since entoprocts obviously do not possess a coelom during any stage of their life cycle (Nielsen, 1971; Emschermann, 1996).

Urochordates do not form the typical mesodermal coeloms (enterocoels) that characterize various other deuterostomes (Nielsen, 1995; Welsch, 1995; Presley et al., 1996), although the pericardium of ascidians and thaliaceans has been considered by some to be a coelom (Godeaux, 1990; Burighel \& Cloney, 1997).

The tardigrades have been reported to possess every type of body cavity distinguishable in invertebrates including an enterocoel, schizocoel, pseudocoelom, hemocoel, and mixocoel (Nelson, 1982; Kinchin, 1994). Resolution of this problem has been hindered by the lack of convincing embryological data, as well as by terminological ambiguities associated with body cavities. No coeloms have been reported in postembryonic stages, and the adult body cavity answers the definition of a primary body cavity with all tissues and organs lined by a basal lamina (Dewel et al., 1993). Nevertheless, Meglitsch \& Schram (1991), and Zrzavý et al. (1998) score presence of a coelom, whereas Eernisse et al.'s (1992) character scoring is apparently contradic- tory; it scores both presence of a coelom and a pseudocoelom (E15, E21, E23). Additional discussion of the tardigrade body cavity is provided under Developmental or morphogenetic mode of the coelom.

The possession of a coelom by the myzostomids is disputed. Rouse \& Fauchald (1995, 1997), Fauchald \& Rouse (1997), Rouse (1999), and Westheide (1996) accept the presence of a coelom or coelomoducts in myzostomids. In contrast, Haszprunar (1996a), Zrzavý et al. (1998), and Eeckhaut et al. (2000) argue against these interpretations. Eeckhaut (1995) could not confirm the coelomic nature of the genital ducts, and since this is the most recent study on the internal ultrastructure of myzostomids, it is reasonable to conclude on the basis of current information that myzostomids lack a coelom.

Haszprunar'(1996a) introduced a character into metazoan cladistics coding for the eucoelomate condition. This character is basically a finer subdivision of histologically defined coeloms. The coelom is associated with the body wall muscles, and acts primarily in locomotion in the eucoelomate phyla. This means that at least part of the coelomic lining is differentiated into either true muscle cells or myoepithelial cells that form part of the body wall. Haszprunar proposed this character as typical of the echiurans, sipunculans, annelids, and the lophophorate and deuterostome phyla. Haszprunar claimed that nemerteans and molluscs are not eucoelomate because in these groups the coeloms are not clearly associated with the body musculature and locomotion. The subsequent analyses by Zrzavý et al. (1998, 2001) and Zrzavý (2003) incorporated this character, but some interesting changes in scoring happened between these studies.

The coeloms in the molluscs and nemerteans have nothing to do with body wall musculature in contrast to the scoring of ZI11 and ZII19 (however, see Turbeville, 2002 for hints of a connection between body wall muscles and the coelomic circulatory vessels in an interstitial nemertean). The chaetognath coelomic lining contributes muscles to the body wall in contrast to the scoring of Z32 and ZI1 1 (Shinn, 1997). The coelomic lining in the ectoprocts may contribute muscles to the body wall (Mukai et al., 1997; Nielsen, 2001). Finally, postembryonic stages of the panarthropod phyla do not 
have body wall muscles that are part of intact coelomic cavities, in contrast to the scoring of ZI11 and ZII19. Also, there is no evidence that suggests that the transient coeloms in the embryonic stages of the onychophorans and arthropods ever form part of the body wall muscles (Bartolomaeus \& Ruhberg, 1999). It is surprising to find that Zrzavý et al. (2001) ignored all this information by scoring these phyla as "? $\mathrm{s}$ " for ZII1. The changes in scoring for the nemerteans and molluscs in Zrzavý (2003) are puzzling. They are "treated here as eucoelomate" (Zrzavý, 2003: 77), despite the fact that they are not according to the adopted definition. However, Zrzavý (2003) does not explicitly state the definition for ZII19. From the comments that Zrzavý provides, and in view of the scoring of the nemerteans and molluscs as eucoelomate it appears that ZII19 actually codes for a histologically defined coelom. However, in that case the arthropods and onychophorans would also have to be scored as present for ZII19 because during embryogenesis they possess transient coeloms that may be retained in the adult as the nephridial sacculi (see above and Bartolomaeus \& Ruhberg, 1999).

What is the phylogenetic significance of a structurally defined coelom in the Metazoa? The phylogenetic analysis of Haszprunar (1996a) suggests the independent evolution of a histologically defined coelom in Mollusca, Nemertea, and the clade (Sipuncula (Echiura (Polychaeta Clitellata))), whereas the analysis of Zrzavý et al. (1998) suggests the homology of the coelom in these taxa, and convergent evolution of a coelom in Panarthropoda, Chaetognatha, Priapulida, and the clade (Lophophorata Deuterostomia). The analysis of Meglitsch \& Schram (1991) implied the monophyletic origin of a coelom as a synapomorphy of the Coelomata. However, the incorrect scoring of entoprocts has an important effect, so that a re-analysis after rescoring of entoprocts results in the collapse of the clade Coelomata. Nielsen (1995, 1998b) argued that coeloms in protostomes and deuterostomes are convergent, but the phylogenetic analysis of Nielsen et al. (1996) does not provide unambiguous support for or against the monophyletic origin of a coelom.

The threefold convergence of the histologically defined coelom in Haszprunar (1996a) is interpreted on the following topology:
(Nemertea (Entoprocta Mollusca)(Myzostomida (Sipuncula(Echiura(Polychaeta Clitellata))))

This supposes the primary lack of a coelom in entoprocts and myzostomids. However, it is equally parsimonious to suggest homology of coeloms in these taxa with the secondary loss of a coelom in the myzostomids and entoprocts. The same ambiguity results from the analysis of Rouse (1999): homology of the coelom in the euspiralians implies secondary loss of the coelom in the entoprocts and myzostomids, or primary lack of a coelom in the entoprocts implies convergence of the coelom in the nemerteans and other euspiralians. Indeed some authors favor the derivation of the entoprocts from coelomate ancestors (Bergström, 1997; CavalierSmith, 1998), while others suggest it is more likely that the entoprocts are derived from a small noncoelomate ancestor (Haszprunar, 1996a). The diversity of current phylogenetic hypotheses (either morphological, molecular, or combined) for the placement of entoprocts defies any clear resolution. The same holds true for the myzostomids. Their traditional positioning within the Polychaeta has received cladistic support from the analyses of Rouse \& Fauchald (1997), which is in line with a secondary absence of a coelom. Their nesting within the coelomate neotrochozoans in Zrzavý et al. (1998) supports the same conclusion. However, their placement in the analysis of Haszprunar (1996a) is ambiguous as to the evolutionary significance of their body cavity organization, whereas the analysis of Zrzavý et al. (2001) appears to support their primary non-coelomate nature (see Jenner, in press, for a critical discussion of recent attempts to place Myzostomida within the Metazoa).

Phylogenetic analyses of metazoan relationships based primarily on $18 \mathrm{~S}$ rRNA/DNA data have yielded a range of different results and interpretations for body cavity evolution that can broadly be classified into three categories:

1) Homology of coeloms in early studies, that suffer seriously from taxon sampling defects (especially the exclusion of non-coelomates), and the incorporation of fast clock organisms, such as $C$. elegans (Field et al., 1988; Raff et al., 1989; Ghiselin, 1989; Turbeville et al., 1992; Telford \& Holland, 1993; Raff et al., 1994; Philippe et al., 1994; 
Winnepenninckx et al. 1995b). Consequently, the phylogenetic support for a monophyletic (Eu)Coelomata reported in these studies 'cannot be regarded as reliable (see also Jenner, 2000).

2) Studies that do include a broader sampling of taxa, but in which phylogenetic inferences are constrained by the influence of unconvincing interpretations from the morphological literature. These analyses are strongly biased towards accepting coelomate monophyly and the overall homology of coeloms on the basis of unsubstantiated ad hoc assumptions, and they derive non-coelomate body organizations from coelomate precursors (Winnepenninckx et al., 1995a; Mackey et al., 1996; Balavoine, 1997, 1998; Balavoine \& Adoutte, 1998; Aguinaldo \& Lake, 1998; Adoutte et al., 1999, 2000; Knoll \& Carroll, 1999; Dewel, 2000). A typical and the clearest illustration of such reasoning is the first comprehensive molecular study of pseudocoelomate phylogeny by Winnepenninckx et al. (1995a). They write (p. 1136) "The most fundamental evolutionary implication of multiple origins of pseudocoelomates is that the body cavity type is of less phylogenetic significance than previously considered...and implies that body cavities that appear to be pseudocoeloms could easily be derived by the modifications of existing eucoeloms." This is particularly revealing since both cladograms in Winnepenninckx et al. (1995a) favor the alternative interpretation of the primitive lack of a coelom and the independent evolution of coeloms in the protostomes and deuterostomes (Aguinaldo \& Lake, 1998; p. 884-885 adopt the same falacious reasoning). In this case as in the other studies cited above, non-coelomate bilaterians are assumed to be modified coelomates, a conclusion supposedly supported by several morphological findings.

The most convincing morphological evidence suggesting the evolution of non-coelomate organisms from coelomate ancestors involves an analogy with interstitial polychaetes that reduced the coelom with decreased body size, e.g., Fransen (1988), and Rieger (1994b). However, the evidence is hardly compelling because in many compact-bodied interstitial annelids the organization and polarity of the somatic and visceral muscular systems remain distinct from that found in acoelomate and pseudocoe- lomate phyla (Fransen 1988; Bartolomaeus, 1994). The dwarf male of Bonellia viridis potently illustrates that extreme size reduction need not lead to complete loss of coelomate characters (Schuchert \& Rieger, 1990). Ruppert (1991a) also argued that some aschelminths may be derived from ancestors with perivisceral coeloms because the nematomorphs, some nematodes, priapulids, and acanthocephalans have body cavities resembling perivisceral coeloms; the somatic mesoderm resembles nonstratified hypertrophied myoepithelium found in various coelomate invertebrates. However, with the exception of the meiofaunal priapulid, Meiopriapulus fijiensis, in none of these taxa has a mesodermal myoepithelium or peritoneum been convincingly demonstrated. Ruppert's hypothesis does imply actual inheritance of a coelom in non-coelomates. Lorenzen (1985) similarly derives the pseudocoelomates from coelomate ancestors, with direct inheritance of the coelom. In contrast, Rieger (1986a, $1994 \mathrm{a}, \mathrm{b}$ ) derived the acoelomates and pseudocoelomates by progenesis from an ancestor with a biphasic life cycle with a macroscopic coelomate adult and a microscopic acoelomate/pseudocoelomate larva. This would explain the striking diffences between larval and adult body organization and at the same time explain the similarities between classic primary larvae and non-coelomate adults, e.g., muscle system, body cavity, excretory system, etc. However, actual reduction of a coelom is not implied by this hypothesis because the non-coelomates derived from ancestral larval stages before ontogenetic differentiation of the coelom.

3) Studies that include a broader sampling of taxa, support the potential convergence of coeloms or the primary lack of coeloms in various non-coelomate bilaterians. However, these papers do not necessarily explicitly discuss the evolution of body cavity organization in their papers, e.g., Eernisse (1997), Littlewood et al. (1998), Ruiz-Trillo et al. (1999), Giribet \& Ribera (1998), Zrzavý et al. (1998), Giribet \& Wheeler (1999), and Peterson \& Eernisse (2001). Although these comprehensive studies do not.yield a completely resolved bilaterian clade, a conservative parsimony estimation indicates that convergent evolution of a structurally defined coelom is more likely than its overall homology across 
Bilateria (see also Jenner, 2000). However, the analyses of Giribet et al. (2000) and Zrzavý et al. (2001) do not yield unambiguous conclusions about the homology of coeloms.

What are we at this point to conclude? Comprehensive morphological and molecular cladistic studies have not as yet supplied convincing support for a monophyletic origin of the coelom, but this conclusion is based upon the assumption that gain and loss of a coelom are equally probable. With respect to the acoelomate worms, parsimony appears to support the homology of the nemertean coelomic cavities (circulatory vessels, rhynchocoel) with the coeloms of the neotrochozoans. The secondary loss rather than the primary absence of a coelom in the platyhelminths and gnathostomulids is not strongly supported. Ultimately, a definitive conclusion will depend on a robust hypothesis of relationships indicating the distribution of non-coelomate and coelomate taxa and a fuller understanding of the relative probabilities of character gains and losses (if that can be achieved at all).

\section{2) Ontogenetic source}

The embryonic origin of the mesoderm has been considered as especially relevant for the consideration of the homology of secondary body cavities. On the basis of comparative anatomy a classical distinction has been made between ectomesoderm (ectomesenchyme, mesectoderm) and endomesoderm (mesendoderm or "true" mesoderm), and these being derived from ectoderm and endoderm respectively (Hyman, 1951a; Salvini-Plawen \& Splechtna, 1979). A note on terminology is in order here. Ectodermally derived mesoderm is variably referred to in the literature as ectomesenchyme or ectomesoderm. Careful use of terms is necessary since these terms are used by different authors to connote very precise homology proposals especially regarding the homology of mesoderm in the Bilateria and the mesogloea in cnidarians and ctenophores. Since mesogloeal cells do not form true organs (although the rather arbitrary definition of an "organ" might call this argument into question) it was suggested that designating them as a germ layer, i.e., mesoderm, would be inappropriate (Siewing, 1969). Consequently, ectomesenchyme became the pre- ferred term for cells located between the epidermis and gastrodermis. For different perspectives on terminology see Hyman (1951a), Salvini-Plawen (1998a), Salvini-Plawen \& Splechtna (1979); Siewing $(1969,1977)$; Starck \& Siewing (1980); Hall (1998). It should be noted, however, that most recent papers have settled with ectomesoderm and have not been explicitly concerned with issues of terminology. In this paper I use the terms ectomesoderm and ectomesenchyme interchangeably.

The differentiation between ecto- and endomesoderm has long been regarded as very important for resolving invertebrate relationships. Various authors have argued for the phylogenetic distinctness of ectomesoderm and endomesoderm with the former evolving before the latter (Wilson, 1898; Hatschek, 1911; Hyman, 1951a; Siewing, 1969; Salvini-Plawen \& Splechtna, 1979). Ectomesenchyme is widespread in spiralians and diploblasts, and was thought to be totally lacking in deuterostomes. In addition, the conviction that ectomesoderm solely contributed to larval structures led to its designation as larval mesoderm. Recently, zoologists have turned away from this rigid division, and now generally admit an underlying unity between endo- and ectomesoderm (Ruppert, 1991a; Nielsen, 1995; Hall, 1998) as is reflected in the cladistic character codings of mesodermal characters, e.g. N27, and Z17. Recent cell lineage studies, in particular on the presumed basal spiralian platyhelminths and nemerteans, confirm and extend the results from earlier studies that indicate that the ecto- and endomesoderm are not strictly separate sources of larval and adult tissues, respectively (confirmed also by unpublished observations of $\mathrm{P}$. Damen for the gastropod $\mathrm{Pa}$ tella). Both may contribute to larval and adult structures, and frequently mix in the development of particular structures. Both larval and adult tissues are composed of a mixture of ecto- and endomesoderm in a variety of spiralians (Boyer et al., 1996a, 1998; Boyer \& Henry, 1998; Henry \& Martindale, 1998a, 1999). The presence of both ecto- and endomesoderm, derived from opposing ventrally and dorsally positioned blastomeres respectively, is posited for the spiralian ancestor (Boyer et al., 1996a, 1998; Henry \& Martindale, 1999). Ectomesoderm in spiralians may be derived from either the first (leeches, possibly echiurans), second (molluscs, 
polychaetes, nemerteans, and polyclads), or third quartet micromeres (molluscs, nemerteans, echiurans, and polychaetes) (Boyer 't al., 1996a; Boyer \& Henry, 1998; Henry \& Martindale, 1998a, 1999). However, modern cell lineage tracing methods are necessary to confirm these observations.

Interestingly, some profound evolutionary changes have occurred in different taxa in the relative contribution of ecto- and endomesoderm to adult spiralian tissues. The mesoderm of the prosobranch gastropod Paludina (Viviparus) has been reported to originate either as outpocketings of the archenteron, or exclusively as ectomesoderm (Verdonk \& Biggelaar, 1983). Although the need for reinvestigation is obvious, the second interpretation has attained widespread approval (Raven, 1966; Hyman, 1967; Webber, 1977; Salvini-Plawen \& Splechtna, 1979; Nielsen, 1995). Similarly, all mesodermal derivatives of the polychaete Eunice may be exclusively composed of ectomesoderm (Nielsen, 1995).

The deuterostomes are traditionally regarded as completely lacking ectomesoderm (Salvini-Plawen \& Splechtna, 1979; Rieger, 1996a), but even in the deuterostomes we can trace the ectodermal origin of various mesodermal organs such as the pericardium in enteropneusts and asteroids (Ruppert, 1991a). Although based on older data, the reports of ectodermal cells that ingress into the blastocoel of various phoronid species (see Bartolomaeus, 2001) also warrant a detailed reconsideration. However, whether exclusive homology can be maintained between ectomesodermal structures throughout the animal kingdom is doubtful. The ectomesodermal origin of structures in spiralians is firmly rooted in corresponding cell lineages (first, second, and third quartet micromeres), but the deuterostomal ectomesodermal derivatives mentioned by Ruppert (1991a) do not share these origins. For example, the pulsatile vesicle that develops from ectomesenchyme in the enteropneust tornaria larva (Ruppert $\&$ Balser, 1986) and the ectomesodermal structures developed from the second or third micromere quartet in the trochophore larva of gastropod molluscs (Verdonk \& Biggelaar, 1983; Dictus \& Damen, 1997 ) are both formed in the larva and may both contribute to adult structures (the pericardium and foot, respectively). However, there is nothing that suggests a single phylogenetic origin of these ectomesodermal structures. So far, the lack of convincing homology between the ectomesenchyme of nonbilaterians and bilaterians, or protostomes and deuterostomes, leaves the phylogenetic significance of the fundamental separation of ecto- and endomesoderm unresolved.

It should be noted that current textbooks provide a definition of endomesoderm that is inaccurate and which may lead to confusion with respect to the phylogenetic significance of endomesoderm. It is stated that endomesoderm is derived from endoderm (Meglitsch \& Schram, 1991; Gilbert \& Raunio, 1997), implying that mesoderm derives from already differentiated endoderm. A developmentally more accurate characterization of endomesoderm that is in line with recent investigations of the source of mesoderm in diverse animal phyla is rooted in detailed cell lineage (Boyer et al., 1996; Boyer \& Henry, 1998; Henry \& Martindale, 1998a, b, 1999; Henry et al., 2000; Martindale \& Henry, 1999). Endomesoderm would then be defined as mesoderm deriving from cells that will produce both endodermal and mesodermal progeny. Similarly, ectomesoderm is mesoderm deriving from cells that produce both ectodermal and mesodermal progeny. For example, it was recently found that the acoel platyhelminth, Neochildia fusca, produced only endomesoderm (Henry et al., 2000). This conclusion was based on the fact that mesoderm derives solely from the progeny of third duet macromeres, cells that only produce mesoderm and endoderm. However, the third duet macromeres arise from second duet macromeres that also produce ectodermal offspring. Therefore, the last split of developmental competence should be applied as an unambiguous criterion to diagnose ecto- or endomesoderm. These considerations have some consequences for character scoring (see under Muscles).

For the purpose of this discussion, I will provisionally accept all mesoderm to be homologous, coincident with a least restrictive topological definition as the middle body layer (Ruppert, 1991a), while further awaiting evidence hinting at the nature of the primitive form(s) of mesoderm. Especially needed are detailed cell lineage studies on the origin of mesoderm in various aschelminths. Since mesoderm as a secondary germ layer is a 
quintessentially inductive product (Hall, 1998), molecular developmental analysis of mesoderm induction processes may hold the key to uncovering the evolutionary unity or true diversity of mesoderm (see also below).

Alternatively, one may distinguish different sources of mesoderm in the Bilateria. The most common distinction made in recent cladistic studies is between archenteron derived mesoderm, and mesoderm derived from one or more cells near the blastopore (N27, N28, NI25, NI26, Z17, Z20, ZI2, ZI10, S33, S34, P36, P38, B18, B22, M19, Ca1).

\section{Character coding}

N27; NI25; S33; Z17: mesoderm formed from 4dcell, blastopore rim, or ectomesoderm $\mathrm{a} / \mathrm{p}$

N28; NI26; S34; Z20; ZI10; ZII9; B22: mesoderm formed from archenteron $\mathrm{a} / \mathrm{p}$

P36: endomesoderm derived from gut $\mathrm{a} / \mathrm{p}$

M19: ectomesoderm/4d mesoderm

Cal: $4 \mathrm{~d}$ mesoderm/mesoderm from archenteron (endomesoderm)

B18: mesoderm arises from mesentoblast (primitively the $4 d$ cell) a/p
E5; P38; ZI2; ZII7: 4d (endo)mesoderm a/p H37; Z19: entomesoblast (4d/2d) a/p

\section{Character scoring and phylogenetic significance}

Sørensen et al. (2000) doubted the presence of mesoderm as a germ layer in the ctenophores (see discussion above), therefore scoring '?' This conclusion is supported by the fact that during the development of neither the cnidarians nor the ctenophores a true mesodermal cell sheet is present. This reasoning also explains why Peterson \& Eernisse (2001) scored mesoderm absent in the ctenophores (P34, P36, P38), but this scoring is in apparent conflict with an earlier study that supported endomesoderm as a synapomorphy of ctenophores and bilaterians (Peterson \& Davidson, 2000), and the scoring for P35 (presence of endomesodermal muscles). The scoring conflicts hinge upon the definition of a germ layer. If a germ layer is strictly defined (classical definition) as an epithelium, then the ctenophores lack a third germ layer. However, when a broader definition is accepted that is not restricted to an epithelium but that may also encompass individual cells, then the ctenophores do

Table 17. Scoring conflicts of archenteron derived mesoderm.

\begin{tabular}{|c|c|c|c|}
\hline & Absent & Present & $?$ \\
\hline Ctenophora & P36 & $\mathrm{Z} 20 ; \mathrm{N} 28 ; \mathrm{NI} 26$ & S34 \\
\hline Pterobranchia & & $\begin{array}{l}\text { Z20; ZII9 (Hemichordata); } \\
\text { N28; NI26; S34; Cal }\end{array}$ & $\mathrm{P} 36$ \\
\hline Phoronida & S34 & Z20; ZII9; N28; NI26; S34; ZI10; Cal & \\
\hline Ectoprocta & Z20; ZII9; N28; NI26; S34; ZI10 & & P36; Cal \\
\hline Cephalorhyncha & $\mathrm{ZI10} ; \mathrm{P} 36$ & & Z20; ZII9; N28; NI26; S34 \\
\hline Tardigrada & & $\mathrm{Z} 20$ & N28; NI26; S34; P36;ZI10;ZI19 \\
\hline Syndermata & Z110; ZII9 (Acanthocephala) & & $\begin{array}{l}\text { Z20; ZI19 (Rotifera); N28; NI26; } \\
\text { S34; P36 (Rotifera) }\end{array}$ \\
\hline Gnathostomulida & $\mathrm{Z} 20$ & & S34; ZI10; ZII9; NI26; S34 \\
\hline Cycliophora & S34 & & $\mathrm{Z} 20 ; \mathrm{Zl10}$ ZII9; P36 \\
\hline
\end{tabular}

Table 18. Scoring conflicts of mesoderm derived from $4 \mathrm{~d}$, blastopore rim, or ectomesoderm.

\begin{tabular}{|c|c|c|c|c|}
\hline & Absent & Present & & $?$ \\
\hline Chaetognatha & N27; NI25; Z17, Z19; ZI2; ZII7; P38 & & & S33 \\
\hline Loricifera & S 33 & & & N27; NI25; Z17; Z19; ZI2; ZII7; P38 \\
\hline Tardigrada & $\mathrm{Z17} ; \mathrm{Z19}$; P38 & E5 & & N27; NI25; S33; ZI2; ZII7 \\
\hline Acanthocephala & N27; NI25; Z17; Z19; ZI2; ZII7 & & & $\mathrm{S} 33$ \\
\hline Acoela & P38; ZII7 & $\mathrm{Z17} ; \mathrm{Z19}$ & $\cdot$ & \\
\hline Gnathostomulida & & $\mathrm{Z} 17 ; \mathrm{H} 37$ & - & NI25; S33; P38; Z12; E5; Z19; ZII7 \\
\hline
\end{tabular}


possess endomesodermal cells (as do bilaterians). Because ctenophores clearly possess cells that later develop such mesodermal structures as different muscles, I support this latter interpretation.

The most complete account of pterobranch development is that for Rhabdopleura normani (Lester, $1988 \mathrm{a}, \mathrm{b})$. The embryo is largely filled with a mass of yolk-filled mesenchymal cells that give rise to the coeloms and only during late metamorphosis to the gut. An archenteron was not observed, tentatively justifying the scoring of P36 as opposed to the other studies. However, an invaginated archenteron has supposedly been reported for Cephalodiscus (Nielsen, 2001), but I have not been able to consult the original sources.

An archenteral origin of at least ingressed individual mesodermal cells has been reported for some phoronids which supports the scoring of most studies except S34 (Kumé, 1968b; Emig, 1990; Zimmer, 1991, 1997), but there remains conflict between different reports of phoronid mesoderm (Lüter, 2000 ). An origin of mesoderm from the vegetal plate of the embryo before gastrulation is reported for other species. Freeman \& Martindale (2002) recently found that mesoderm in the phoronids develops at the boundary between ecto- and endoderm, and that it can form from both ectodermal and endodermal cells.

Mesoderm has not been reported to originate from the archenteron in ectoprocts as is reflected in the scoring of most cladistic analyses, but the exact source of the mesoderm remains a mystery, which also justifies the scoring of P36 and Cal (Nielsen, 1971, 1990; Reed, 1991; Lüter, 2000).

The only cephalorhynch phylum for which some embryological data exists is Priapulida. The mesoderm does not appear to form from the archenteron (Nørrevang \& Van der Land, 1989; Nielsen, 2001), nor has a mesentoblast been observed, but I have not been able to consult the few available original works on priapulid embryology. With nothing known about the embryology of either the kinorhynchs or loriciferans, the scoring of a '?' for mesoderm source is currently the best option for cephalorhynchs.

Since the first embryological studies on the Tardigrada, the origin of their mesoderm has been problematic. Although the scoring of archenteron derived mesoderm in Zrzavý et al. (1998) is frequently presented in the literature, a recent study by Eibye-Jacobsen (1996/1997) revealed that most accounts of tardigrade embryology are based on questionable interpretations [just three papers with original observations from the late $19^{\text {th }}$ to the early $20^{\text {th }}$ century]. Mesodermal origin from the archenteron could not be confirmed and remains as yet unknown (see also under Developmental or morphogenetic mode), also against E5 that scored presence of entomesoblast for tardigrades.

E5 scored $4 \mathrm{~d}$ mesentoblast present for the arthropods. The sources of mesoderm are not in agreement with those in the trochozoans, i.e., mesoderm arises not from mesentoblast $4 \mathrm{~d}$ (Anderson, 1973; Siewing, 1979; Scholtz, 1997; Nielsen, 2001; Gerberding et al., 2002; Hertzler, 2002; Wolff \& Scholtz, 2002).

$\mathrm{H} 37$ and Z19 scored the myzostomids as possessing a $4 \mathrm{~d}$ mesentoblast, but in view of the absence of cell lineage data ZI2 properly rescored them as '?.'

Archenteron derived mesoderm has not been reported for the syndermates (ZI10), but the precise cellular source of the mesoderm remains unknown, as reflected in the scoring of most analyses.

The source of the mesoderm in the gnathostomulids is unknown (Mainitz, 1989), contra H37, $\mathrm{Z17}, \mathrm{Z1} 19$ and Z20. Two presumable mesoblasts were observed by Riedl (1969) but neither their identity as descendants from a $4 \mathrm{~d}$ mesentoblast, nor their derivatives could be confirmed.

The source of mesoderm is unknown for $\mathrm{Cy}$ cliophora, contra S34.

If the origin of the chaetognath coeloms is a reliable proxy for estimating the source of the mesoderm, then mesoderm is formed from the archenteron (Alvariño, 1990; Nielsen, 2001), against S33. However, see discussion below on the current crude criteria for establishing mesoderm source.

Although ectomesoderm and a $4 \mathrm{~d}$ mesentoblast are not found in the acoels (contra Z19), it is possible that mesoderm derives from the blastopore (Z17) (Henry et al., 2000). However, it is also possible that the mesoderm becomes only distinguishable after gastrulation has internalized the descendants of the third duet blastomeres that will eventually produce mesoderm. 
A note on character coding is in order here. HaId, $\mathrm{H} 37$, L52, and Z19 are characters that unite cells $4 \mathrm{~d}$ and $2 \mathrm{~d}$ as the source of (endo)mesoderm in various spiralians. This inappropriately unites a true source of mesoderm (4d) with an important source of ectoderm $(2 \mathrm{~d})$. The $2 \mathrm{~d}$ cell is the primary somatoblast in neotrochozoans and provides a major contribution to the ectoderm, e.g., the shell and foot in the molluscs, the segmented ectoderm in polychaetes, and larval ectoderm and peripheral nervous system in polyclads (Schroeder \& Hermans, 1975; Verdonk \& Biggelaar, 1983; Boyer et al., 1998). Only in the nemerteans has $2 d$ been reported to contribute to mesoderm (Boyer et al., 1996a; Boyer \& Henry, 1998). The derivation of mesoderm from the $2 \mathrm{~d}$ cell in the acoel platyhelminths, e.g., Haszprunar, (1996b) is not supported by recent cell lineage analysis, which instead suggests that all acoel mesoderm derives from third duet macromeres (Henry et al., 2000).

Different analyses largely agree on the phylogenetic significance of mesoderm derived from $4 \mathrm{~d}$, blastopore ridge, or ectomesoderm. Nielsen et al. (1996) and Nielsen (2001) support this character as a synapomorphy for Gastroneuralia + Ectoprocta, excluding Syndermata and Chaetognatha, or Chaetognatha only, respectively. Zrzavý et al. (1998) and Sørensen et al. (2000) support it as a synapomorphy for Gastroneuralia minus Chaetognatha and Ectoprocta. In contrast, there appears to be some disagreement about the phylogenetic significance of archenteron derived mesoderm. Nielsen et al. (1998), Nielsen (2001), Peterson \& Eernisse (2001), and Zrzavý et al. (1998) suggest that the archenteron is the plesiomorphic source of mesoderm in the Metazoa, whereas Sørensen et al. .(2000), Zrzavý (2003) and Zrzavý et al. (2001) support archenteron derived mesoderm as a synapomorphy for Brachiopoda, Phoronida, and Deuterostomia (also including Chaetognatha in the first two studies).

It should be realized that these differences in the phylogenetic significance of archenteron derived mesoderm are actually quite small. The alternatives appear to hinge upon a different scoring for Ctenophora, which is the sister group to Bilateria in these analyses (not included in Zrzavý et al., 2001). The scoring of archenteron derived mesoderm for the ctenophores favors the optimization of this char- acter as the plesiomorphic source of mesoderm in the Metazoa. Alternatively, when the ctenophores are scored absent for archenteron derived mesoderm, then the character is favored as a synapomorphy for a clade of lophophorates (minus Ectoprocta) and deuterostomes. An exception is the analysis by Peterson \& Eernisse (2001), which scored ctenophores as lacking archenteron derived mesoderm, but which could support the archenteron as the plesiomorphic mesoderm source by virtue of the basal positions of taxa with this type of mesoderm in all major bilaterian clades.

One may be tempted to conclude that the widespread adoption of characters that dichotomize the source of mesoderm as either the archenteron, or the $4 \mathrm{~d}$ mesentoblast, blastopore ridge, or ectomesoderm, accurately reflects a unified understanding of the evolution of mesoderm sources in the Metazoa. I have serious reservations about this. The interplay of three key issues needs to be confronted: 1) the criteria used to define characters and character states, 2) the nature of mesoderm as an induction product, and 3) heterochrony in the timing of mesoderm origin.

The recent cladistic analyses at least universally agree on considering archenteron derived mesoderm typical, if not necessarily apomorphic, for deuterostomes: "mesoderm is in all cases formed from the endoderm (archenteron), never from the blastopore lips as in the protostomes" (Nielsen, 2001: 375). This conclusion is actually very misleading.

The main problem is that different criteria are used to diagnose the origin of mesoderm in the protostomes and deuterostomes, and these criteria maintain the existence of arbitrarily dichotomized characters or character states. The origin of the mesoderm within the protostomes has been best established for the spiralians and nematodes. The exact cellular source of mesoderm in these taxa is known through detailed cell lineage studies. For most spiralian phyla, for example, it has been clearly established that the mesoderm takes origin from the $4 \mathrm{~d}$ mesentoblast, which may be distinguished as early as the 24-cell stage embryo (caenogastropods) (Biggelaar \& Haszprunar, 1996). In all these taxa, the cells that are specified to become mesoderm can be recognized early in embryogenesis in an area around or close to the future blastopore, 
typically before gastrulation commences, and thus before an archenteron is formed. In the following discussion spiralian ectomesoderm is not considered, only $4 \mathrm{~d}$ mesoderm and blastopore edge mesoderm, which are considered closely similar.

Recent cladistic analyses thus score the origin of mesoderm in these phyla according to the onset of mesoderm specification. In contrast, the source of deuterostome mesoderm is diagnosed by a wholly different criterion, namely the first sign of a morphological differentiation in the mesoderm. In taxa such as the echinoderms and enteropneusts this differentiation occurs with the evagination of coelomic sacs. However, as is also discussed for brachiopods under Developmental or morphogenetic mode, equating the onset of coelomogenesis with the timing of mesoderm origin is not straightforward, and this linking has led to meaningless character scorings in recent cladistic analyses. Lüter (2000: 25) clearly illustrates this confusing perspective: "Although formation of coelomic compartments differs among echinodermate taxa ... enterocoely $\ldots$ is the only way initial mesoderm is formed in Echinodermata." Coelomogenesis and mesoderm origin are, however, not the same. Applying coelomogenesis and mesoderm specification as different criteria to score mesoderm source in the deuterostomes and gastroneuralians, respectively, only serves to create a false impression of their fundamental difference.

Since the mesoderm is an induction product (that does not automatically exclude a role for segregated factors in cell fate specification), it is critical to know the exact timing of this induction when we construct a character on the basis of the source or origin of mesoderm. For those deuterostomes for which experimental and cell lineage data exist, principally several echinoderms and ascidians, e.g. Davidson et al. (1998), Davidson (2001), Sweet et al. (1999), it is beyond doubt that mesoderm specification is already established during the blastula stages, long before the first signs of morphologically differentiated mesoderm become apparent.

Similarly, Freeman \& Martindale (2002) showed that mesoderm in the phoronids is induced at the boundary between the ectoderm and endoderm, and that mesoderm may develop both from endoderm and ectoderm. Consequently, this argues against the clean dichotomy of mesoderm source in the deuterostomes and protostomes as maintained by Nielsen (2001, 2002a).

Under this new perspective it becomes immediately obvious that a quintessential "mesoderm from archenteron" taxon such as Echinodermata is actually a "mesoderm from blastopore edge" taxon. For example, when we consider that "stereotype for deuterostome development" (Pearse \& Cameron, 1991: 572), the sea urchin, we must conclude that the blastomeres that are specified as mesoderm can already be precisely identified on a blastula fate map. The same conclusion can be drawn when we study the fate map of an ascidian cleavage stage embryo. The prospective mesoderm cells in the blastulae of these taxa are located around the blastopore edge before involuting into the archenteron where they will eventually form the source for morphologically differentiated mesodermal derivatives. A focus on specification rather than morphological differentiation may also lead to different character scorings for vertebrates, even though embryonic cell lineages play a far less prominent role in vertebrate development than in embryos from most other phyla. Vertebrates are universally scored as having archenteron derived mesoderm, and lacking ectomesoderm, but mesoderm induction commences well before gastrulation with prospective endoderm inducing presumptive ectoderm cells to become mesoderm (Kessler \& Melton, 1994; Hall, 1998).

This novel perspective may also provide a broad explanatory umbrella that can accommodate a cell type that is otherwise often considered an echinoderm oddity: the mesodermal mesenchyme cells that contribute various cell types to the larval body plan. Secondary mesenchyme cells develop from the tip of the growing archenteron during gastrulation, and they are found in members of all living classes. Primary mesenchyme cells develop from the vegetal plate before gastrulation starts, but they are often considered a peculiarity for the echinoids, ophiuroids, and holothuroids (Holland, 1991; Chia \& Walker, 1991; Hendler, 1991; Pearse \& Cameron, 1991; Smiley et al., 1991; Wray, 1997; Davidson et al., 1998). The presence of primary mesenchyme in these taxa appears to correlate with the presence of larval calcareous spicules or ossicles, and at least 
for the echinoids and ophiuroids it is known that the spicules are produced by ingressed primary mesenchyme cells. The crinoids, which are the acknowledged sister group to the other living groups of echinoderm (Littlewood et al., 1997; Janies, 2001), are not reported to have primary mesenchyme, but crinoid gastrulation (Hyman, 1955; Holland, 1991) appears to start with a unipolar ingression of cells that might be interpreted as primary mesenchyme. 1 therefore think that the presence of primary mesenchyme, or pre-gastrulation mesoderm, in the ground pattern of Echinodermata (at least the living groups) remains a viable hypothesis.

The distinct characters or character states in the recent cladistic analyses merely formalize an arbitrary distinction that is maintained by application of different diagnostic criteria of mesoderm origin in different taxa. I consider the perspective elaborated here to be better grounded in observations on metazoan development. This perspective could serve as the basis for a revised character coding and scoring that would clearly contradict those adopted in recent cladistic analyses. The new perspective primarily argues against the exclusive scoring of mesoderm derived from around the blastopore for gastroneuralians, removing a potential gastroneuralian synapomorphy, and raising the possibility that it represents a synapomorphy for a more inclusive clade, possibly the Bilateria or Acrosomata. The possibility of the latter hypothesis hinges upon the scoring of ctenophores as possessing archenteron derived mesoderm rather than mesoderm derived from the blastopore region. (Nielsen et al., 1996; Nielsen, 2001; Zrzavý et al., 1998). Again, this character scoring formalizes an arbitrary distinction by giving a later ontogenetic state precedence over an earlier one. Ctenophore mesoderm is developed from oral micromeres that are formed at the oral pole, which is comparable to the vegetal or blastoporal pole in bilaterians. Later during gastrulation the oral micromeres shift from their initial position in the area where the blastopore will form, to a position lining the archenteron (Martindale \& Henry, 1997). In this context, a recent statement by Martindale \& Henry (1999) deserves some comment.

Until very recently the traditional interpretation of mesogloea in ctenophores either questioned homology with bilaterian mesoderm (Rieger, 1996a, b), or indicated a possible phylogenetic link with spiralian ectomesoderm (Siewing, 1977; SalviniPlawen \& Splechtna, 1979). Recent studies have shifted these interpretations towards the ctenophores either possessing true mesoderm that unites them with the deuterostomes (Nielsen, 1995), or possessing endomesoderm. This latter interpretation would provide a possible synapomorphy of either the ctenophores and protostomes (Martindale \& Henry, 1997), or the ctenophores and bilaterians (Martindale \& Henry, 1998, 1999; Peterson \& Davidson, 2000). However, the comparability of ctenophore and bilaterian mesoderm is complicated by the lack of consensus on the relationship between the oral-aboral axis of diploblasts and the antero-posterior axis of bilaterians (Goldstein \& Freeman, 1997; Henry \& Martindale, 1998b). In the first modern fate mapping study of the phylum, Martindale \& Henry (1999) claimed that ctenophore endomesodermal derivatives originate from the animal and not the vegetal pole of the ctenophore embryo, which is apparently unique in the Metazoa. If this is so, then comparability of blastopore edge derived mesoderm in the bilaterians and ctenophores becomes problematic. However, Martindale \& Henry (1999) place great emphasis on the site of polar body extrusion as the marker of the embryonic animal pole, indicating that the ctenophoran blastoporal pole is shifted 180 degrees relative to that of most bilaterians. Instead, I would accept as a more likely alternative that the site of the blastopore is comparable across the eumetazoans, with a secondary shift in the site of polar body extrusion occurring in the ctenophores (Siewing, 1977). This interpretation is indirectly supported by experimental data for the ctenophores. Under normal, undisturbed conditions, the site of first cleavage and polar body formation coincide. When the site of polar body formation is changed, as may sometimes occur either under natural circumstances, or by centrifugation experiments, the antero-posterior axis of the embryo is developed in relation to the site where the first cleavage is initiated (this becomes the oral pole) independent of the position of the polar bodies, a situation also found in Cnidaria (Freeman in Goldstein \& Freeman, 1997). Thus there appears to be little reason for not comparing the blastoporal pole of ctenophoran and bilaterian embryos. 
Finally, the possibility of heterochronic shifts in mesoderm development during evolution must be confronted if our goal is to code and score meaningful phylogenetic characters. Because mesoderm induction takes place while the embryo undergoes cleavage and/or gastrulation movements, a small change in the timing of mesoderm induction could mean the difference between mesoderm located around the blastopore ridge, and mesoderm derived from presumptive endoderm invaginated into the archenteron. Finding out whether such heterochronic changes are possible could inform us whether it would be better justified to code separate characters, or merely different character states for mesoderm derived from the blastopore region (including 4d), and the archenteron. The former coding method hypothesizes logical independence of nonhomologous traits, whereas the second method hypothesizes that the observed variation may be homologous, but different, manifestations of the same character that may be transformed into each other. The majority of cladistic analyses adopt the coding of separate characters (N27, NI25, S33, Z17, N28, NI26, S34, Z20, ZI10, B22, P38, ZI2, B18), while only two studies reflect the variation as alternative character states (Ca1, E7). Can we decide which is the best supported alternative?

Interestingly, the currently upheld dichotomy between blastopore ridge and archenteron derived mesoderm has not always been interpreted as fundamental. Already MacBride (1895) and Wilson (1898) suggested that the fundamental source of bilaterian mesoderm was the archenteron, either located in one to a few cells (gastroneuralians), or more cells (deuterostomes). Simple differential growth of the archenteron could bridge the gap between these opposite organizations, and both authors indicated a belief in an evolutionary connection between these different sources of mesoderm. The possibility of evolutionary changes in the developmental timing of the origin of mesoderm (heterochrony) can, for example, be generally illustrated for the gastropods and other spiralians (Biggelaar \& Haszprunar, 1996; Ponder \& Lindberg, 1997; Guralnick \& Lindberg, 2001), and specifically for a shift for mesoderm derivation from the blastopore edge to mesoderm derived from the archenteron in the mollusc Chiton polii (Raven, 1966), and between early (blastopore edge) and late (archenteron) development of skeletogenic mesenchyme as indicated by the sea urchin Eucidaris (Davidson et al., 1998). The distinction between primary and secondary mesenchyme cells in echinoderms may also be a reflection of heterochronic changes. It is at least suggestive to regard the observed variation in the timing of mesenchyme development (before or during gastrulation) within the different echinoderm taxa (Strathmann, 1988) as the results of heterochrony. Also, simply looking where mesoderm is located at different times during the development of a single animal indicates no fundamental distinction between mesoderm from the blastopore region or the archenteron. For example, before the left and right mesentoblasts in the scaphopod mollusc Dentalium start to produce endodermal and mesodermal célls, they remain part of the archenteron lining during "gastrulation (Verdonk \& Biggelaar, 1983), a situation similar to that claimed typical of deuterostomes. Similar examples for the presence of blastopore edge mesoderm in the deuterostomes are given above. Taken together, I believe it is better to create a single character to describe the different sources of metazoan mesoderm (with the possible exception of spiralian ectomesoderm), rather than to construct separate characters.

Although the incorporation of the timing of cell specification processes into our character definitions will be important for a proper understanding of the evolutionary significance of different mesodermal sources, recognizing specified mesoderm before it is morphologically differentiated poses a practical problem. To recognize mesoderm before it is morphologically differentiated, we need the help of molecular markers for mesodermal cells, or detailed cell lineage tracing studies. Such studies are necessary if we want to depart from the currently used crude morphological criteria, e.g., the formation of coelomic sacs, for determining the cellular source of mesoderm.

In summary, different criteria for diagnosing the origin of mesoderm in the protostomes (specification) and the deuterostomes (coelomic pouching) have lead to artificially dichotomous character coding and scoring that hinders a proper understanding of mesoderm origins. Studies of mesodermal induction in deuterostomes show that mesoderm 
may be determined in a comparable region near the blastopore as in the gastroneuralians (Arendt \& Nübler-Jung, 1997). In addition, a demonstration of the possibility of heterochronic shifts in the timing of mesoderm specification in various taxa, and the significant proportion of taxa with unknown origins of mesoderm scored in recent studies (Nielsen et al., 1996, Zrzavý et al., 1998; Sørensen et al., 2000), leads us to conclude that mesoderm derived from the blastopore ridge is not a reliable synapomorphy for the gastroneuralians.

\section{3) Developmental or morphogenetic mode}

"It is reasonable, then, to regard an enterocoelous method of coelom formation as symplesiomorphic in Bilateria..." -Dewel (2000: 43)-

"The enterocoelous condition of deuterostomes apparently is a myth..." -Bergström (1997: 11)-

Schizocoely and enterocoely are generally presented as the two principal alternative morphogenetic modes for coelom formation in the Bilateria and characteristic for protostomes and deuterostomes, respectively (Hyman, 1951a; Ruppert \& Barnes, 1994; Rieger, 1996a; Brusca et al., 1997). This traditional division is maintained in recent large scale morphological cladistic analyses of the Metazoa (Brusca \& Brusca, 1990; Meglitsch \& Schram, 1991; Eernisse et al., 1992; Zrzavý et al., 1998) that characterize different taxa by one or the other morphogenetic mode. Such a clear dichotomy is perhaps simplistic because it does not accurately reflect the variation seen between or within bilaterian taxa (or even individuals). The difficulty of maintaining this clean dichotomy is effectively demonstrated if one consults different parts of the most recently published English language compendium on the comparative embryology of the metazoans (Gilbert \& Raunio, 1997). In this work, Brusca et al. (1997) characterize the deuterostome branch of their phylogeny by possession of an 'enterocoelous coelom,' while Whittaker (1997: 371) writes on "...schizocoely, a more deuterostomal and vertebrate characteristic."

A related but not identical division derives mesoderm either from epithelial or non-epithelial cells.
Mesoderm of epithelial origin forms by evagination of pouches from the archenteron, and coincides with coelom formation through enterocoely (Ruppert \& Barnes, 1994; Salvini-Plawen \& Splechtna, 1979). In contrast, non-epithelial mesoderm can be derived from both ectoderm and endoderm and is generally referred to as (ecto- or ento-) mesenchyme. Hyman (1951a: 19) summarized: "Since in fact all methods of mesoderm origin except the enterocoelous are more or less mesenchymal, no very sharp line can be drawn between them." Embryonic mesenchyme may either be retained as mesenchymal mesodermal tissue in the adult, or reorganized into coelomic epithelium by undergoing a mesenchymal-to-epithelial transition, a process referred to as schizocoely. However, it should be noted that not all schizocoelous mesoderm originates from non-epithelial mesoderm. Some species of polychaetes, for example, may derive their mesoderm from epithelially organized mesoderm bands (Turbeville, 1986; Rieger, 1986a). At this time not enough species from different phyla have been investigated by ultrastructural methods to derive phylogenetic information from the tissue organization of the embryonic mesodermal bands in teloblasticans (schizocoelians).

The syntecocoel (Salvini-Plawen \& Splechtna, 1979; Salvini-Plawen \& Bartolomaeus, 1995), or neocoel (Remane, 1963a), or mesenchymal coelom (Hyman, 1951a), is usually not discussed and is never scored in cladistic studies, although it is an equally distinctive developmental mode of coelom formation as entercoely and schizocoely. It refers to coeloms lined by an epithelium arising through a coalition of individual mesenchymal cells, such as the molluscan pericardium and the phoronid preoral hood coelom (protocoel). However, it should be noted that a recent ultrastructural investigation of the ontogeny of phoronid coeloms by Bartolomaeus (2001) could not confirm the presence of a protocoel. Instead, the presence of a large extracellular cavity is suggested in the actinotroch preoral hood.

It may appear surprising that various authors regard the mechanically rather simple processes of schizocoely and enterocoely as so fundamentally distinct that they cannot even conceptualize how one developmental mode could have given rise to 
another, for example Inglis (1985), Moore \& Willmer (1997). These authors favour the convergent evolution of coeloms over their homology with the possibility of transformations between their developmental modes. Other authors have devised schemes of linear evolutionary transformations between schizocoely and enterocoely with opposite polarities of change such as a change from schizocoely to enterocoely in Hadzi (1953), Salvini-Plawen \& Splechtna (1979), Salvini-Plawen (1982), Ivanov (1988), Dzik (1993), and Christoffersen \& Araújode-Almeida (1994), or from enterocoely to schizocoely in Remane (1963b), Siewing (1972), Cavalier-Smith (1998), Knoll \& Carroll (1999), and Dewel (2000).

Considered as mechanical processes, schizocoely and enterocoely do not represent complex features strictly associated with coelom formation. Enterocoely is mechanically identical to processes of outpouching, folding, or invagination that are common in the development of many epithelial structures in metazoans, such as embolic formation of the archenteron, neurulation movements in chordates, formation of pharyngeal pouches in vertebrates, or the formation of the stomodeum and proctodeum in many phyla. Schizocoely is the hollowing of a solid cell mass and is likely to be effected, at least partially, by the secretion of fluid between the cells, e.g., schizocoely of polychaete coeloms by fluid accumulation, although the source of the fluid has not been ascertained (Bartolomaeus \& Ruhberg, 1999). Secretion is a basic feature of many cell types, and schizocoelic formation of cavities can be seen in the development of a host of structures in many taxa across the Metazoa, from the development of a spacious blastocoel in mollusc embryos (Verdonk \& Biggelaar, 1983), through the formation of the gonocoels in nematomorphs (Lanzavecchia et al., 1995), to the hollowing out of the entocodon in hydrozoan gonozoids to form the subumbrella of the medusa (Boero et al., 1998).

If we accept that schizocoely and enterocoely as purely (and clearly defined) mechanical processes that do not constitute features of great complexity, then it becomes crucial to precisely qualify the spatio-temporal characteristics of these mechanisms. It is the link of these processes to a source of mesoderm that is important for a proper understanding of their phylogenetic significance. This realization holds the key for resolving some problematic character scorings, notably for brachiopods and phoronids (see discussions below).

\section{Character coding}

B19: schizocoel (E17: schizocoel lined by peritoneum) $a / p$

B23: body tripartite and enterocoelous (E18: enterocoel lined by peritoneum) $\mathrm{a} / \mathrm{p}$

BI77: no body cavity formed during embryogenesis/schizocoelous coelom/enterocoelous coelom M52; Z27: schizocoel/enterocoel

Ca19: schizocoely/enterocoely/highly modified enterocoely

RI2, R125: coelom (schizocoel) a/p

$\mathrm{C} 1$ : schizocoel/anterior three pouches enterocoelic/ all vestiges of schizocoëlic metamery are lost Hyman (1951a), Ruppert \& Barnes (1994): mesenchymal vs. epithelial origin of coelom

\section{Character scoring and phylogenetic significance}

The enterocoelic origin of tardigrade coelomic cavities is an example of a poorly documented character that nevertheless, and due to paucity of new investigations, has become ensconced as textbook fact and a cladistic data matrix entry (Pollock, 1975; Nelson, 1982; Meglitsch \& Schram, 1991: M52). Nielsen $(1995,1997)$ expressed serious reservations about the reliability of the few published works on tardigrade embryology, and Eibye-Jacobsen (1996/ 1997) could not confirm the enterocoelic nature of tardigrade coeloms. However, a schizocoel has also not been confirmed (BI77). At present the mode of coelomogenesis in the tardigrades remains uncertain.

Modern accounts of onychophoran development are still primarily based on the observations of Manton (1949). Following Manton, Siewing (1969), Anderson (1973), Nielsen (1995), and Scholtz (2002) describe and depict the origin of solid mesodermal bands in onychophorans and the subsequent formation of pairs of coelomic cavities by schizocoely. Walker (1995) confirms schizocoely of somites in Opisthopatus cinctipes.

I accept schizocoely in the arthropods, for which 
Table 19. Scoring conflicts of mode of coelomogenesis.

\begin{tabular}{|c|c|c|c|c|c|}
\hline & $\begin{array}{c}\text { Enterocoely } \\
\end{array}$ & Schizocoely & $\begin{array}{l}\text { Polymorphic } \\
\text { (schizocoely/ } \\
\text { enterocoely) }\end{array}$ & $\begin{array}{l}? \\
\text { (unknown) }\end{array}$ & $\begin{array}{l}\text { Proposed } \\
\text { scoring }\end{array}$ \\
\hline Tardigrada & M52 & $\mathrm{BI} 77$ & & $\mathrm{Z} 27$ & $?$ \\
\hline Onychophora & & M52, RI2, W59, BI77 & & $\mathrm{E} 17, \mathrm{E} 18, \mathrm{Z} 27$ & Schizocoely \\
\hline Arthropoda & & M52, RI2, W59, BI77 & & $\mathrm{Z} 27$ & Schizocoely \\
\hline Brachiopoda & $\begin{array}{l}\text { M52, Z27 (articulates), } \\
\text { C1, Ca19, BI77 }\end{array}$ & Ca 19 (lingulaceans) & E17, E18 & $\begin{array}{l}\text { Z27 (inarticulates), } \\
\mathrm{Ca} 19\end{array}$ & $?$ \\
\hline Phoronida & $\mathrm{C} 1, \mathrm{E} 18, \mathrm{Ca} 19, \mathrm{BI} 77$ & M52 & & $\mathrm{Z} 27$ & $?$ \\
\hline Ectoprocta & $\mathrm{BI} 77$ & M52 & & $\mathrm{Ca} 19,227$ & $?$ \\
\hline Urochordata & $\mathrm{Cl}, \mathrm{Z27}, \mathrm{BI} 77$ & E17 (Chordata) & & & ? or schizocoely \\
\hline Cephalochordata & $\mathrm{C} 1, \mathrm{Z27}, \mathrm{M} 52, \mathrm{BI} 77$ & E17 (Chordata) & & & $\begin{array}{l}\text { Enterocoely or } \\
\text { polymorphic }\end{array}$ \\
\hline Vertebrata & $\mathrm{Cl}, \mathrm{BI} 77$ & E17 (Chordata) & & $\mathrm{Z} 27$ & Enterocoely or? \\
\hline Pterobranchia & $\mathrm{Cl}, \mathrm{Z} 27, \mathrm{~B} 23, \mathrm{~B} 177$ & & & M52 & Schizocoely or? \\
\hline Nemertea & & M52, E17, BI77 & & $\mathrm{Z} 27$ & Schizocoely \\
\hline Pogonophora & & E17, M52, RI2 & $\mathrm{Cl}$ & $\mathrm{Z} 27$ & $?$ \\
\hline
\end{tabular}

Anderson (1973) summarized the classical support. However, new studies of onychophoran and arthropod coelomogenesis will be of considerable interest to illuminate the lingering problem of the homology or analogy of metamerism in onychophorans, arthropods, and annelids.

Brachiopods have been scored for all possible modes of coelomogenesis throughout almost a century of reports (Lüter, 2000). A resolution of their true mode(s) of coelomogenesis is of considerable importance considering the conflicting phylogenetic placements of brachiopods and phoronids suggested by morphological and molecular sequence data (rDNA and Hox genes) (Lüter \& Bartolomaeus, 1997). Morphological analyses typically support an affinity of the brachiopods with the deuterostomes, which frequently, but not universally, exhibit enterocoely (the protostome relationship supported by the analysis of Peterson \& Eernisse, 2001 is an exception), and molecular data unambiguously places them among the protostomes which are typically schizocoelous. I know of no confirmed instances of enterocoely in the protostomes, except perhaps for the unique mode of 'enterocoely' reported for chaetognaths and articulate brachiopods; Kapp (2000, Long \& Stricker (1991).

A process that resembles typical enterocoely, in which the archenteral epithelium evaginates to form the rudiments of the coelomic sacs, has been reported for articulate brachiopods only. This mode of coelomogenesis is usually called modified enterocoely, and it is characterized by downwardly growing cellular curtains that subdivide the archenteral lumen into a central future gut lumen flanked by two lateral coelomic sacs (Long \& Stricker, 1991). This is the strongest indication of enterocoely in the brachiopods, but recent studies by Freeman (1993) and Lüter (2000) could not confirm this mode of coelom formation. Instead, Freeman reported that mesoderm delaminates from the dorsal-anterior region of the archenteron, and Lüter's data supports schizocoely (contra Lüter's own interpretation). Below I discuss Lüter's definitions of schizocoely and enterocoely in detail. The presence of schizocoely is supported by descriptions for the embryology of inarticulate brachiopods as well (Hyman, 1959; Kumé, 1968a; Chuang, 1990; Long \& Stricker, 1991; Zimmer, 1997), but convincing pictorial support appears weak (Hyman, 1959; Kumé, 1968a). Enterocoely in one form or another is not reported for inarticulates.

Nielsen (1991) reported that mesoderm originates as a single thin sheet of cells in the inarticulate craniid brachiopod Crania. The mesoderm then spreads between ecto- and endoderm, a process that might be interpreted as highly modified enterocoely. However, a clear evagination of the archenteron, i.e., the key feature of enterocoely, was not observed, Moreover, Freeman (2000) could not confirm Nielsen's results. Freeman (2000) instead found that 
the mesoderm originates through ingression of individual cells from the archenteron (also reported for the inarticulate Discinisca; Freeman, 1999). This mode of mesoderm formation excludes enterocoely as the process of coelomogenesis and is at least consistent with schizocoely or syntecocoely.

The recent investigations of brachiopod coelomogenesis by Lüter (2000) merit special attention. Being acutely aware of the danger of terminological confusion with respect to coelom formation, Lüter (2000) attempted to give clear definitions of schizocoely and enterocoely. Unfortunately, he adds to the confusion by combining mode of coelomogenesis and source of the mesoderm in his definition of enterocoely. Lüter (2000: 23) states that schizocoely is "only a mechanism," while in contrast enterocoely "describes both a developmental process and the origin of the mesodermal cells involved." This definition of enterocoely is not a problem as long as there is an obligatory link between the archenteron as the mesoderm source and the process of enterocoely, an interpretation frequently promoted by textbooks. For example, Kozloff (1990: 835) writes: "[deuterostome] mesoderm is derived from the archenteron, so they are enterocoelous." However, this strict correlation is refuted in exactly those articulate brachiopods that Lüter (2000) has chosen to study. He clearly describes that in both investigated species the mesoderm originates as a solid mass that later hollows out. Consequently he writes that "without looking at the origin of the mesoderm cells, one could conclude that proliferation of a solid mesodermal anlage ... is to be considered schizocoely" (Lüter, 2000: 26). Yet, instead he concludes that the actual mechanism of coelom formation is "of secondary interest," and that "as long as the mesoderm ... originates from the archenteral epithelium the mode of coelom formation is to be, described as enterocoely" (Lüter, 2000: 23). Lüter clearly gives primacy to the source of the mesoderm as the final arbiter on the mode of coelomogenesis. I think this line of reasoning only leads to confusion. It is very important to apply clear definitions, because not to do so may have major phylogenetic consequences.

Recent authors, perhaps justifiably so, tend to emphasize the phylogenetic significance of mesoderm source over that of mode of coelomogenesis
(Lüter, 2000; Nielsen, 2001). This leads to grouping of the brachiopods and phoronids with the deuterostomes (based on the archenteron as the source of mesoderm, but see discussion under Ontogenetic source). However, an emphasis on mode of coelomogenesis might instead support a position of these phyla among schizocoelous protostomes such as the neotrochozoans, a position at least more compatible with $18 \mathrm{~S}$ rRNA/DNA phylogenies. However, I do not wish to defend here the phylogenetic primacy of either mesoderm source, or mode of coelomogenesis. I merely want to stress the importance of clear definitions, and to caution against unwanted constraining effects that improper definitions may have on phylogenetic reasoning.

In conclusion, I am reluctant to unambiguously score a particular mode of coelomogenesis as plesiomorphic for the brachiopods based on my study of the recent investigations of brachiopod embryogenesis (I have been unable to study most of the older sources). Despite the unambiguous determination that mesoderm derives from the archenteron or its base (both in the inarticulates and articulates), I tentatively conclude that reports of enterocoely, i.e., epithelial folding, appear unsupported, in contrast to M52, Z27, C1, Ca 19, Nielsen (1991, 2001), Lüter (2000), and Bartolomaeus (2001). This would remove one potential synapomorphy for brachiopods and deuterostomes. However, the archenteral origin of mesoderm would still support a relationship to deuterostomes.

How distinct is the mesodermal origin in the brachiopods and, for example, neotrochozoans? To answer this question, we first need to estimate which of the living brachiopod taxa is most likely to be representative of the primitive mode of development. Recent morphological and molecular phylogenetic analyses indicate strong support for a monophyletic Articulata, but the monophyly of the inarticulates (lingulaceans, discinaceans, and craniaceans) is less robustly supported. In fact, both morphological and molecular (SSU rDNA) phylogenetic analyses provide some support for a paraphyletic inarticulate grade at the base of a monophyletic Articulata (Carlson, 1995; Williams et al., 1996; Cohen et al., 1998; Cohen, 2000). It would thus be most profitable to consider inarticulate development. Interestingly, the mesodermal cells are 
found to arise either at the base of the archenteron at the junction between ectoderm and invaginating endoderm (Glottidia and Discinisca: Freeman, 1995, 1999), or from various regions of the invaginating archenteron where they ingress into the blastocoel as individual cells (Crania: Freeman, 2000) and in Lingula solid mesodermal cell masses flank the archenteron (Chuang, 1990; Long \& Stricker, 1991; Zimmer, 1997). These patterns show some striking resemblances to the origin of mesoderm in neotrochozoans. For example, in polychaetes and molluscs the $4 \mathrm{~d}$ mesentoblast is located at the posterodorsal side of the archenteron, in close connection with it (Anderson, 1973; Verdonk \& Biggelaar, 1983). Mesodermal bands are also formed as solid cell masses that cavitate by schizocoely to form the coeloms. The presence of individual mesodermal cells or mesodermal cell masses in inarticulates is at least compatible with schizocoely (or with syntecocoely) and not with enterocoely, but more studies are definitely needed. The origin of mesodermal cells from an already invaginated archenteron as observed in Crania and articulates may be derived within the Brachiopoda and may simply be the result of a shift in the timing of mesoderm origination to somewhat later stages. This also has a counterpart in the neotrochozoans. For the scaphopod Dentalium it has been observed that the left and right mesentoblasts remain part of the archenteral lining before they start dividing to form enteroblasts and mesoblasts (Verdonk \& Biggelaar, 1983).

These similarities appear to indicate that a fundamental gap in the source of mesoderm and mode of coelomogenesis does not exist between the brachiopods and various protostomes. This may be a significant conclusion considering the placement of brachiopods among the protostomes in molecular phylogenetic studies, and it underscores the potential importance of considering heterochrony for understanding the relationships between apparent differences in mesoderm source and mode of coelom formation (see also under Ontogenetic source and Budd \& Jensen, 2000). Naturally, more modern embryological studies are imperative for an eventual definitive conclusion. It is possible that the reports on both the different sources of mesoderm and the different modes of coelomogenesis are reflections of a highly variable developmental program in the brachiopods, or that reported variations (e.g. Nielsen, 1991, Freeman, 2000, and Grobe, 2000 on Crania) are merely artifacts resulting from the use of different analytical techniques (light or electron microscopy and none of the available studies used a mesoderm specific marker to recognize mesoderm).

The (modified) enterocoely reported for phoronids in Emig (1982), Brusca \& Brusca (1990, 2003), Eernisse et al. (1992), Carlson (1995), Williams et al. (1996), and Bartolomaeus (2001) refers to the archenteron as the source of the mesoderm and ultimately the coelom. As is discussed above for brachiopods, the source of these problematic scorings is a conflation of mode of coelomogenesis with source of the mesoderm. Although phoronid mesoderm arises from the archenteron, genuine outpocketings of the archenteron, i.e., enterocoely, have not been observed for any phoronid (Bartolomaeus, 2001: 136 cites a study by Malakhov \& Temereva, 1999 for a report of a "pouching of entodermal material" which would hint at true enterocoely. Unfortunately, I have not been able to consult that publication). In fact, the phoronids exhibit various modes of coelom formation during the ontogeny of a single individual, and between different species, depending on which coelom (proto- meso- or metacoel) is considered (Emig, 1982, 1990; Zimmer, 1991, 1997). However, Bartolomaeus (2001) concluded that a protocoel is absent in the phoronids.

It is also tempting to conclude that a priori hypotheses of the phylogenetic placement of the phoronids have colored the interpretation of phoronid mesoderm and coelom development to a certain extent. For example, the modified enterocoely reported in some works fits nicely with the deuterostome affinity of the lophophorates suggested in those same works (Brusca \& Brusca, 1990; Carlson, 1995; Williams et al., 1996). In general, the lophophorates are frequently favored as intermediary taxa bridging the gap between the protostomes and deuterostomes, as is illustrated in various works including Hyman (1959), Salvini-Plawen (1982, 1998a, b), and Christoffersen \& Araújo-de-Almeida (1994).

In Ectoprocta one may observe syntecocoely, schizocoely (of tentacle coelom), and unique modes of coelomogenesis (Reed, 1991; Mukai, 1982). Typical enterocoely (outpouching of the archenteron) 
has not been observed, in contrast to the scoring of BI77. The obscure origin of the mesoderm, and the unique and variable modes 'of coelomogenesis in the ectoprocts (Mukai, 1982; Reed, 1991; Zimmer, 1997) have led some authors to doubt the homology of ectoproct coeloms with those of other bilaterians (Nielsen, 1971, 1995; Meglitsch \& Schram, 1991). Nevertheless, the ectoprocts do possess a histologically defined coelom, albeit frequently with only incomplete coelomic linings (Mukai et al., 1997). At this time our limited understanding of mesodermal differentiation in ectoprocts does not allow any unambiguous character scoring.

The presence of a coelom has not yet been unequivocally confirmed for urochordates (see under Secondary body cavity, coelom for references), with the pericardium as the best candidate. The appearance of the pericardium within a solid mass of mesodermal cells in thaliaceans (Godeaux, 1990) might be suggestive of schizocoely, but this information is not sufficient to infer with any certainty the mode of urochordate coelomogenesis. Although mesoderm cells may be derived from the archenteron wall, enterocoely has never been reported (Schaeffer, 1987). The observed character scorings thus appear to be unfounded.

All somites of amphioxus are derived from the archenteron, and enterocoely of at least the first pairs of coelomic cavities has been well established for approximately a century (Schaeffer, 1987; Presley et al., 1996; Holland et al., 1997; Stach, 2000; Nielsen, 2001). The more posteriorly formed somites, however, are more solid and the cavities that can be discerned in them may arise through schizocoely.

It is difficult to unambiguously determine the characteristic mode of vertebrate coelomogenesis (Schaeffer, 1987; Ghiara, 1995; Nielsen, 2001). The textbook deuterostome character of enterocoely is not generally present in most major vertebrate taxa (Romer \& Parsons, 1986), and vertebrate enterocoely is by some authors denied altogether (Ghiara, 1995). Nevertheless, a mode of enterocoely similar to that of cephalochordates is at least observed for the anterior coeloms of the phylogenetically basal lampreys, and this may tentatively be accepted as a vertebrate ground pattern state (Goodrich, 1958; Schaeffer, 1987; Presley et al., 1996; Nielsen, 2001).
Summarizing the data available for Chordata as a whole, it can be concluded that their characterization as strictly schizocoelous (E17) is clearly incorrect.

In contrast to the enteropneusts, coelom formation has not been exhaustively studied in the pterobranchs, and consequently the mode of coelomogenesis remains uncertain (Hyman, 1959; Hadfield, 1975). For example, Bergström et al. (1998) claim schizocoely, Brusca \& Brusca $(1990,2003)$ and Zravy et al. (1998) score enterocoely, and Meglitsch \& Schram (1991) score unknown mode of coelomogenesis. Since embryological studies have not convincingly demonstrated enterocoely, the scoring of this character in recent cladistic studies is incorrect, and must have a different explanation. The demonstration of the archenteron as the source of mesoderm and the prevalent phylogenetic assumption of enterocoely as a quintessential deuterostome synapomorphy have likely biased the interpretation of pterobranch coelomogenesis towards accepting enterocoely (see earlier for a similar explanation of the problematic scorings of enterocoely in brachiopods and phoronids). Schizocoely of mesoand metacoels and the pericardial cavity, on the other hand, has been demonstrated (Lester, 1988a, b), but this information has so far not entered cladistic data matrices.

Lateral vessels in the nemertean Prosorochmus americanus develop by schizocoely from longitudinal solid mesodermal tissue strands (Turbeville, 1986). This remains the sole recent study supporting schizocoely in nemerteans, justifying the scoring for M52 and E17.

Over thirty years of debate about the origin of pogonophoran (including vestimentiferan) mesoderm and mode of coelomogenesis have not been able to resolve the controversy (Ivanov, 1988; Gardiner \& Jones, 1994; Rouse \& Fauchald, 1995; Southward, 1993, 1999; Salvini-Plawen, 2000). Notwithstanding, great phylogenetic significance has been attributed to the mode of coelomogenesis as the ultimate arbiter for either protostome or deuterostome affinities of the pogonophorans in both past (Ulrich, 1972) and recent studies (Christoffersen \& Araújo-de-Almeida, 1994; Malakhov et al., 1997). Those studies that score schizocoely for pogonophores (Meglitsch \& Schram, 1991; Eernisse et al., 
1992; Rouse \& Fauchald, 1995) all place them among the protostomes. Reports for the perviate (frenulate) pogonophorans range from species with either schizocoely or enterocoely to species that exhibit both modes in the embryology of a single individual, and for the vestimentiferans schizocoely has been reported. Consequently, recent cladistic studies have scored the pogonophorans in diverse ways. Perhaps surprisingly, none of these studies scored exclusively enterocoely despite that enterocoely seems to be on a stronger evidential footing than schizocoely (Ivanov, 1988; Soutward, 1993; Salvini-Plawen, 2000). I will not attempt to 'resolve' the issue here by arbitrary allegiance to one of the alternatives, and I would rather conclude that it is not possible to assign a typical or primitive mode of coelomogenesis to the pogonophores.

I do not attach great phylogenetic significance to mode of coelomogenesis when separated from the source of mesoderm. This is in agreement with various other studies that tend to downplay the phylogenetic importance of mode of coelomogenesis (Nielsen, 1995; Nielsen et al., 1996; Bergström, 1997; Budd \& Jensen, 2000). Variation within phyla with undoubtedly homologous coeloms, such as in enteropneusts or ectoprocts, or the presence of more than one mode of coelom formation during the ontogeny of different coeloms in a single individual, such as possibly in cephalochordates, support this conclusion. This would also imply that the coding of separate characters for different modes of coelomogenesis is not warranted: different modes of coelomogenesis appear to be "the same but different" (characters states) manifestations of a single homologous character.

\section{$\underline{\text { Coelomocytes }}$}

\section{Character coding}

H29; Z33: coelomocytes a/p

\section{Character scoring and phylogenetic significance}

Haszprunar (1996a) introduced a character on the presence of coelomocytes into metazoan phylogenetics. Although he considered it to be a potential synapomorphy of the eucoelomates (all the protostome, lophophorate, and deuterostome coelomates), his restrictive taxon sampling did not allow this hypothesis to be tested. Coelomocytes were scored present for the Nemertea, Sipuncula, Echiura, and Annelida. Zrzavý et al. (1998) adopted this character with the same scoring, despite their broader taxon sampling which included other eucoelomate phyla. Consequently, coelomocytes were incorrectly scored as absent in taxa such as the pterobranchs, enteropneusts, echinoderms, brachiopods, phoronids, ectoprocts, and possibly cephalochordates (Heinzeller \& Welsch, 1994; Benito \& Pardos, 1997; Herrmann, 1997; James, 1997; Mukai et al., 1997; Ruppert, 1997). Thus, the phylogenetic significance of coelomocytes still remains to be assessed. This will be a challenging task if we want to elevate the definition of coelomocytes above that used in Haszprunar (1996a): 'cells within the coelom.' Coelomocytes come in a variety of forms and functions (including the immune system), and these undoubtedly include several taxon specific types that are not useful for elucidating the phylogenetic relationships between phyla, for example the multicellular coelomic urns of the sipunculans and the holothuroid crystal cells (Rice, 1993; Smiley, 1994).

\section{Nervous system and sensory organs}

\section{Cerebral ganglion, brain}

Anteriorly located ganglion (not necessarily the largest), typically associated with the anterior sense organs, and located (antero-)dorsally to the digestive tract when that is present. Also often referred to as brain or supraesophageal ganglion.

\section{Character coding}

H13; Z237; ZII92: cerebral ganglion a/p HaIIla: central nervous system with paired cerebral ganglion at anterior end $\mathrm{a} / \mathrm{p}$

B194: brain (cerebral ganglia) absent/present, ringlike, encircling proboscis apparatus/present, ringlike, encircling pharynx/present, not ringlike/brain collar-shaped, sitting like a saddle on top of the pharynx/diffuse/brain + dorsal nerve cord N46; S55; ZI46: dorsal nerve concentration/brain behind apical organ/apical pole a/p Z238: dorsal brain, neural concentration $\mathrm{a} / \mathrm{p}$ 


\section{Character scoring and phylogenetic significance}

I will not attempt here to discuss in detail the voluminous literature that deals with the structure and development of the central nervous systems across the Metazoa, but some comments on character scoring are nevertheless necessary. The bilaterian central nervous system is distinguished from that of the non-bilaterian phyla by consisting of an anterior nervous concentration (cerebral ganglion) and one to several main longitudinal nerve cords, but such an organization is by no means universal within Bilateria. Habitually, several different organizations of bilaterian nervous systems are recognized, such as a deuterostome, a chordate, a gastroneuralian, a spiralian, and a cycloneuralian nervous system, e.g., Rieger (1996a), Salvini-Plawen (1998b), and Nielsen (2001). The challenge is to examine whether these distinct configurations of nervous systems can be regarded as variations upon a common theme. The problem with the homology of cerebral ganglia in the different groups is one of the great unsolved issues.

The observed scoring for $\mathrm{H} 13$ and especially Z237 are rather poor reflections of the comparative morphology of invertebrate cerebral ganglia. H13 scored a cerebral ganglion present in the catenulid and rhabditophoran platyhelminths, gnathostomulids, Lobatocerebrum, nemerteans, entoprocts, myzostomids and neotrochozoans. Zrzavý et al. (1998) copied this character from Haszprunar (1996a) and adopted the exact same scoring despite considerable differences in taxon sampling. Several changes in scoring can be made.

First, both $\mathrm{H} 13$ and Z237 misscored Echiura, which lack any anterior or cerebral ganglia (Pilger, 1993; Purschke, 1996; Purschke et al., 2000). However, new detailed immunohistochemical studies of the ontogeny of the central nervous system in Urechis caupo and Bonellia viridis have indicated slight swellings in the antero-lateral parts of the proboscis nerve loop, that might be interpreted as corresponding to the supraesophageal ganglia of annelids (Hessling, 2002; Hessling \& Westheide, 2002).

Second, in view of the fact that a cerebral ganglion is scored for the entoprocts based upon the presence of a frontal ganglion in the larva, Cyclio- phora (scored '?') should be rescored for Z237. All free living stages in the cycliophoran life cycle (female, male, Pandora larva, chordoid larva) have well-developed dorsally located bilobate brains (Funch, 1996; Funch \& Kristensen, 1997), and, although not included in their analysis, Micrognathozoa have bilobed cerebral ganglia as well (Kristensen \& Funch, 2000).

Third, adopting the definition of a cerebral ganglion provided by Haszprunar (1996a), which is not restricted to a particular cellular architecture, it could be concluded that a cerebral ganglion is a synapomorphy for bilaterians, excluding acoelomorphs (see also Haszprunar, 1996b). Many of the phyla that are scored as '?s' in Zrzavý et al. (1998) (Z237) could be rescored as ' 1 ', when such a broad definition of a cerebral ganglion is adopted, including Rotifera, Acanthocephala, Gastrotricha, Tardigrada, Onychophora, and Chaetognatha (Schürmann, 1987; Clément \& Wurdak, 1991; Dunagan \& Miller, 1991; Ruppert, 1991b; Ahlrichs, 1995; Dewel \& Dewel, 1996; Shinn, 1997). This rescoring would even be valid if a more circumscribed definition is adopted. For example, a cerebral ganglion may be defined as an anteriorly located ganglion (anterodorsal to digestive system when that is present), which may be paired or not, with peripherally located perikarya that are distributed more or less evenly around a central neuropile, and which may be surrounded by a basal lamina (subepidermal location) or not (basi- or intraepithelial location).

In taxa with more complex brains, such as the arthropods and cephalopods, the perikarya may be more unevenly distributed across the brain, and the perikaryal layer may also exhibit variation in its degree of development according to the brain region considered (Sandeman, 1982; Joly \& Descamps, 1987; Budelmann, 1995), but the overall structural plan is similar. The cycloneuralian brain, or at least that of the nematoidans and scalidophorans, may be unique (Nebelsick, 1993; Neuhaus, 1994; Schmidt-Rhaesa, 1996; Nielsen, 2001). However, these taxa possess an anterior circumenteric brain (cerebral ganglion) with a distinct regionalizatioñ of perikarya and neuropil.

An anterodorsally located (paired) cerebral ganglion is widely considered as a bilaterian autapomorphy (Brusca \& Brusca, 1990; Ax, 1995; Rieger, 
1996b; Nielsen, 2001), but the observed character scoring for "brain" characters emphasizes the supposed distinctness of protostome and deuterostome (+ lophophorate) brains. This may lead to apparently contradictory character scorings. For example, Z237 scores absence of a cerebral ganglion for deuterostomes and lophophorates, while in contrast Z238 scores the phoronids and deuterostomes as having a dorsal brain (scored as absent in all protostomes). This scoring can also be observed for N46, S55, and ZI46, which score a dorsal brain present only in the deuterostomes and phoronids. The scorings thus assume the evolutionary independence of "dorsal" gastroneuralian and "dorsal" deuterstome + lophophorate brains (and often also the main nerve cords). This contrast between the widespread acceptance of an anterior brain as a synapomorphy of bilaterians on the one hand, and the divergent scoring for protostome and deuterostome brains on the other is in need of an explanation.

Furthermore, Zrzavý et al. (1998) score Ectoprocta as lacking both a cerebral ganglion and a dorsal brain. This ignores the presence of a cerebral ganglion between mouth and anus, a location that is typically regarded as dorsal (Mukai et al., 1997). Nevertheless, neither Nielsen et al. (1996), nor Sørensen et al. (2000) and Zrzavý et al. (2001) score a dorsal nerve concentration in Ectoprocta. Curiously, these studies do unambiguously score echinoderms and enteropneusts (or hemichordates: ZI46) as possessing dorsal brains (Zrzavý, 2003 also scored the echinoderms as possessing a cerebral ganglion). The unique organization of the echinoderm body and nervous system defies any straightforward comparison with any other bilaterian, and recent authors have not even reached any consensus about the orientation of body axes in the echinoderms. Interestingly, two recent attempts to tackle this problem converged upon the same interpretation of echinoderm anteroposterior axis orientation: the $\mathrm{A} / \mathrm{P}$ axis runs from the anteriorly located mouth through the adult coeloms to the posteriorly located right somatocoel (Hotchkiss, 1998;-Peterson et al., $2000 \mathrm{~b}$ ). This model implies that the five ambulacra are outgrowths from the primary body axis, and thus analogous to limbs. This body orientation appears to shed some light on earlier ideas about the circumoral nerve ring as the coordinating center, or brain, of echinoderms by suggesting that this nerve ring is located anteriorly in the body. However, recent studies do not support the idea that the circumoral nerve ring is the coordinating echinoderm "brain" (Cobb, 1995). When the issue is approached morphologically, it can be concluded that the nerves that come off the circumoral ring in taxa such as the ophiuroids, asteroids, and holothuroids are more complexly organized and ganglionated (Byrne, 1994; Chia \& Koss, 1994; Smiley, 1994) than the nerve ring itself. In any case, we must conclude that a clear dorsal (or ventral) brain is not present in the echinoderms, and the character scorings in the cladistic studies should be adjusted accordingly.

The presence of a dorsal brain in the enteropneusts is equivocal at best. A well-defined cerebral ganglion is not present (Bullock, 1965d). The neurochord that is located dorsally in the collar can be excluded as a brain since its functions merely as a through-conducting fiber tract, but the thickened nerve fiber layer that is located in the posterodorsal region of the proboscis has been considered as an equivalent of a brain (Nübler-Jung \& Arendt, 1999). Yet, that does not explain why the adopted character scorings suppose that this morphology is more reminiscent of that in the other deuterostomes rather than that of the gastroneuralians, especially in view of the presence of a well-defined ventral nerve cord in the enteropneusts.

In conclusion, I regard the currently adopted character scorings for brain characters as artificially maintaining a dichotomy between protostomes and deuterostomes (+ lophophorates) while being a rather poor reflection of the actual comparative morphology of bilaterian brains.

A tentative basis for a revised character scoring may be offered by recent gene expression studies that increasingly appear to support a common plan. By extrapolation, a single evolutionary origin of the protostome and deuterostome central nervous systems, including brains and major nerve cords (Arendt \& Nübler-Jung, 1996, 1999a; Nielsen, 1999; Nübler-Jung \& Arendt, 1999). These recent ideas are part of a widespread reconceptualization of dorso-ventral body axis organization throughout the Bilateria that was initiated in the early 1990 s by the discovery of a strikingly similar dorso-ventral 
patterning system that operates during the development of the insects and vertebrates. The probable homology of several of the involved genes in the vertebrates and insects lead to the hypothesis that this dorso-ventral patterning mechanism is retained from Urbilateria, the last common ancestor of the vertebrates and arthropods. These findings fostered the beginnings of a fruitful interdisciplinary synthesis that explored the underlying unity of bilaterian "backs" and "bellies" from an fascinating diversity of perspectives, including developmental genetics, embryonic fate maps and gastrulation patterns, nervous system morphology, pharyngeal and endostyle morphology, and dorsalventral orientation during chordate locomotion (Arendt \& Nübler-Jung, 1994, 1997; Robertis \& Sasai, 1996; Kimmel, 1996; Lacalli, 1996d; Bergström, 1997; Holley \& Ferguson, 1997; Bergström et al., 1998; Nielsen, 1999; Nübler-Jung \& Arendt, 1999; Ruppert et al. 1999; Gerhart, 2000; Biggelaar et al., 2002). At the same time, these developments have led to a remarkable, if somewhat anachronistic, rehabilitation of some of the seemingly more fanciful ideas of the leading $19^{\text {th }}$ century French transcendendal morphologist Étienne Geoffroy Saint-Hilaire, a few years after he resurfaced in the current biological literature in connection with the discovery of homologous Hox genes in vertebrates and insects (Gould, 1986, Nübler-Jung \& Arendt, 1994; Panchen, 2001). This exciting conceptual flux may eventually form the foundation for a cladistic character scoring that is logically fully consistent for all metazoans with respect to dorso-ventral body organization, notably those relating to the position of the components of the central nervous system in bilaterians.

\section{Adult brain derived from or associated with larval apical organ}

In several phyla, notably the neotrochozoans, platyhelminths, and nemerteans, it has been reported that the adult brain develops in close association with the larval apical organ (sometimes with portions of the apical organ actually being incorporated into the brain). However, modern cell tracing methods and electron microscopy will have to reveal the intimacy of this association and the degree of variation between different taxa.

\section{Character coding}

NI45; ZI45: adult brain derived from or associated with larval apical organ $a / p$

N44; S53; Z145: adult brain derived from or associated with larval apical organ/apical pole $a / p$ Z146: larval apical organ incorporated into brain (special case of Z145)

M62: brain not derived from any part of larval apical organ/brain in part derived from larval apical organ, main nerve cord ventral

Table 20. Scoring conflicts for adult brain derived from or associated with larval apical organ.

\begin{tabular}{|c|c|c|c|c|}
\hline & Absent & Present & $?$ & Proposed scoring \\
\hline Cnidaria & M62; ZI45 (diploblasts) & N44; NI45; S53 & $\mathrm{Z} 145$ & Absent \\
\hline Ctenophora & $\begin{array}{l}\text { M62; ZI45; Z146 } \\
\text { (diploblasts) }\end{array}$ & Z145; N44; NI45; S53 & & Absent or? \\
\hline Echiura & S53 & Z145; ZI45; M62 & & Absent or? \\
\hline Myzostomida & & $\mathrm{Z} 145$ & $\mathrm{ZI} 45 ; \mathrm{Z} 146$ & $?$ \\
\hline Nemertea & M62; S53; Z146 & Z145; N44; NI45; ZI45 & 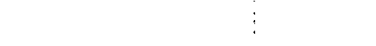 & Present \\
\hline Arthropoda & & $\begin{array}{l}\text { N44; NI45; S53; ZI45; } \\
\text { M62 }\end{array}$ & $\mathrm{Z145}$ & Absent \\
\hline Tardigrada & & M62 & N44; NI45; S53; Z145; ZI45 & Absent \\
\hline Onychophora & & M62 & N44; N145; S53; Z145; ZI45 & Absent \\
\hline Platyhelminthes & M62 & $\begin{array}{l}\text { N44; NI45; S53; ZI45 } \\
\text { (Rhabditophora) }\end{array}$ & $\mathrm{Z} 145$ & Absent \\
\hline Cycliophora & $\mathrm{Z145}$ & & S53; ZI45 & Absent or? \\
\hline Pogonophora & & M62 & $\mathrm{Z} 145$ & $?$ \\
\hline
\end{tabular}




\section{Character scoring and phylogenetic significance}

Although it is commonly reported that cnidarian planula larvae often possess a ciliated apical organ, these are only known from anthozoan planulae, and their presence is probably correlated with their planktotrophic habits (Chia \& Bickell, 1978; Fautin \& Mariscal, 1991; Schäfer, 1996). Because neither the anthozoans, nor any other cnidarians develop an adult brain, N44, NI45, and S53 should be rescored.

The ctenophores possess a complex apical organ, part of which is a concentration of nerve cells which has been referred to as "an 'ancestor' of a cephalic ganglion" (Hernandez-Nicaise, 1991). However, a proper larval stage is never developed, and development proceeds directly to a juvenile stage (cydippid 'larva') that already contains the apical organ that is retained in the adult stage (Komai, 1968; Martindale \& Henry, 1999). Thus, strictly speaking there is no larval apical organ, which contradicts the scoring of Z145, N44, NI45, and S53. On the other hand this scoring can be regarded legitimate because the adult apical organ derives directly from the cydippid apical organ. It is difficult to decide what the potential relationship of the apical organ of the ctenophores could be to the bilaterian brain, but its unique structure suggests that it may be considered as a ctenophoran autapomorphy (Nielsen, 2001).

Because adult echiurans do not possess a welldefined brain (but see the new studies of Hessling, 2002, and Hessling \& Westheide, 2002), Z145, ZI45, and M62 should be rescored. Ax (1999), however, reported that the prostomial nerve ring develops from the "Gehirnanlage in der Episphäre der Trochophora" on the basis of work by Korn (1960). Unfortunately, I have not been able to consult this source, but more recent reviews of echiuran development have not commented upon the origin of the prostomial nerve ring (in contrast to the origin of the ventral nerve cord) (Pilger, 1978, 1997; Davis, 1989).

I am not aware of any studies that have traced the development of the nervous system from larvae to adults in the myzostomids, justifying the scoring of ZI45 and Z146, as opposed to Z145.

Heteronemertean pilidium larvae possess largely non-neural apical organs, that exhibit no relation- ship to the developing adult brain (Lacalli \& West, 1985; Hay-Schmidt, 1990, 2000). In contrast, in the directly developing palaeonemerteans the adult cerebral ganglia develop from a single pair of ectodermal cells located laterally to the apical organ (Iwata, 1960, 1968; Cantell, 1989; Nielsen, 2001). Because direct development appears primitive for the phylum (see elsewhere in this paper), this information supports the scoring of Z145, N44, NI45, and ZI45.

The scoring in various studies of an adult brain derived from a larval apical organ in the panarthropods is very weak. None of these taxa exhibit a larval apical organ, and scoring them as present for a brain derived from a larval apical organ would reduce a specific character to one that could be scored for any taxon with a roughly anteriorly located adult brain.

A close contact between the larval apical organ and the developing juvenile/adult brain has clearly been established for the polyclads that develop through a Goette's larval stage (Ruppert, 1978; Younossi-Hartenstein \& Hartenstein, 2000). Nevertheless, the assumption of this trait in the platyhelminth ground pattern is unlikely given the existence of serious doubts about the primitiveness of indirect development in Platyhelminthes (see under Larva with strongly reduced hyposphere).

Both scorings observed for Cycliophora have some support. A larval apical organ has not been found, justifying the scoring of Z145, but the absence of any data on the embryology of the nervous system alternatively supports the scoring of IS53 and ZI45.

I have not been able to locate any study that supports the scoring of M62 for Pogonophora. The data of Callsen-Cencic \& Flügel (1995) show a connection between the apical organ and the main larval nervous system in settled larvae of Siboglinum, but no information on later stages is available to show whether the adult brain that is developed in the antero-ventral part of the cephalic lobe bears any relationship to the larval apical organ.

The cephalochordates deserve some mention here even though there are no evident scoring conflicts between different studies. All cladistic analyses that included a character coding for the presence or absence of an apical organ have scored Cephalochordata as possessing one (Nielsen et al., 1996; Zrzavý 
et al., 1998; Sørensen et al., 2000; Nielsen, 2001; Peterson \& Eernisse, 2001; N21, Z144, S27, NI19, and $\mathrm{P} 45$ respectively). This scoring is largely based upon the detailed and wide-ranging morphological studies of T. C. Lacalli, part of whose work has focused on elucidating the morphological unity of chordate and non-chordate deuterostome larvae as is seen reflected in various tissues and organs notably larval ciliary bands, nervous systems, and mesodermal derivatives (see Lacalli, 1994, 1996a, b, c, 2001; Lacalli et al., 1990, 1999). Part of his work is a proposal that the frontal eye complex in larval amphioxus and the apical organs that are widespread in marine invertebrate larvae are homologous (Lacalli, 1994, 1996a). This homology proposal has now been incorporated into cladistic data matrices. In order to maintain logical consistency throughout the data matrix, the scoring of an apical organ in Cephalochordata would have to be accompanied by the scoring of the adult brain being derived from or associated with the apical organ because the larval central nervous system, including the frontal eye complex, is retained in the adult. Interestingly, none of the cladistic studies followed this scoring, resulting in scoring conflicts within the different analyses: NI19 versus NI45, N21 versus N44, and S27 versus S53.

Nielsen $(1995,2001)$ states that in the brachiopods "there is no indication that the apical organ becomes incorporated in the adult nervous system" (Nielsen, 2001: 403), and brachiopods are scored accordingly in all the studies considered here. However, the fate of the brachiopod apical organ is at best uncertain. While it appears certain that the apical cilia of various articulate brachiopods are cast off after settlement (Chuang, 1990), this does not necessarily provide any information on the fate of the neuronal cells in the apical organ. In fact, Hyman (1959: 574) and Kumé (1968a) report that cells from the apical plate of articulate larvae contribute to the adult supraenteric (supraesophageal) ganglion in a manner that is "extremely reminiscent of the trochophore larva" (Kumé, 1968a: 277), and HaySchmidt (1992: 203) states that "both the larval apical ganglion and the ventral ganglion must be retained as the adult nervous system" in the inarticulates, but Hay-Schmidt does not supply empirical support for this statement. Clearly, the currently adopted scoring for brachiopods is uncertain at best and potentially erroneous. Until more reliable information is obtained the best solution is to score the brachiopods as '?' for fate of the larval apical organ.

Zrzavý et al. (1998) coded five characters for the presence, ontogenetic fate, and morphology of the apical organ (Z144-148) that reveal several scoring problems. First, Z144 codes for the presence of an apical organ, and misscored non-anthozoan cnidarians and Cycliophora as possessing an apical organ. Second, the scoring of Z145 and Z146 is in conflict for Ctenophora, with the first character implying the development of the adult brain from or near the apical organ, but with the second implying that the adult brain does not develop from the apical organ. However, the ctenophores do not develop separate larval and adult anterior nervous concentrations, and the "larval' apical organ therefore equals the adult 'brain' (see also discussion above). Third, Cycliophora do not possess a larval apical organ and should therefore not be scored as losing it during metamorphosis (Z148).

The phylogenetic significance of this character very much depends upon the adopted character coding. Nielsen et al. (1996), Zrzavý et al. (1998), and Sørensen et al. (2000) coded for adult brains that are derived from, or develop in association with the larval apical organ or apical pole (N44, S53, Z145). Their later cladistic studies (Nielsen, 2001; Zrzavý et al., 2001) revised the character coding so that it no longer encompassed taxa where the adult brain. only develops in association with the apical pole (NI45, ZI45), which merely means that the brain is developed anteriorly. Zrzavý et al. (1998) included a separate character (Z146) strictly coding for adult brains derived from the apical organ. Strictly adopting the first coding strategy would necessitate the rescoring for N44, S53 and Z145 for all phyla that, although lacking an apical organ, develop their brains at the anterior (apical) end of the body. This would yield a character that is merely redundant with respect to a character that simply scores the presence or absence of an anterior cerebral ganglion (see under Cerebral ganglion, brain). The second coding method yields a much more specific, and truly novel, character that should only be scored as present in taxa where the adult 
brain develops from, or in association with the larval apical organ.

In this context, it is important to be able to distinguish between an adult brain that is "derived or initiated from" the apical organ, which is thought to be characteristic for the spiralians (Salvini-Plawen, 2000: 140), or one that develops largely independent of the apical organ, even if they are closely apposed. Modern ultrastructural and cell labeling techniques need to be applied to reassess the differences and similarities of the fate of the apical organ in different phyla because classical observations based on light microscopy may not always be reliable. Even when contact between the apical organ and the developing brain can be discerned, that does not necessarily imply that the larval apical organ has contributed to the developing adult brain. This is illustrated by the development of the brain in the indirectly developing polyclad, Imogine mcgrathi, where brain development is already well underway before one axon bundle establishes contact with the apical tuft cells (Younossi-Hartenstein \& Hartenstein, 2000). This contrasts with claims of the "inductive role" (Salvini-Plawen, 2000: 140) of the apical organ in the differentiation of cerebral ganglia. In fact, although characters such as NI45 and ZI45 unambiguously score the neotrochozoans as developing the adult brain from, or in association with the apical organ, such scorings are actually fairly imprecise.

If we consider the Mollusca, for which the morphology and developmental fate of the larval apical organ have recently been most intensely investigated with ultrastructural and immunocytochemical techniques, especially for the gastropods, no clear message is yet emerging. Although some papers claim that parts of the primary larval nervous system, including the apical organ, may be incorporated into the adult central nervous system, including the cerebral ganglia, e.g., Dickinson et al. (2000), others claim that the apical organ may be closely adjacent to the adult brain (cerebral commissure). These structures nevertheless, either are clearly delineated from each other (Kempf et al., 1997), or they may develop entirely independent from each other (Marois \& Carew, 1997 for the opisthobranch Aplysia; Raineri, 2000). There may be variations between different molluscan taxa, because in con- trast, Lin \& Leise (1997: 184) report that in the prosobranch Ilyanassa the "cerebral commissure was continuous with the neuropil of the apical ganglion." However, later in development one of the most conspicuous changes in the nervous system was the decrease in the size of the neuropil of the apical ganglion and eventually in the postmetamorphic juvenile the complete loss of the apical ganglion. In fact, Lin \& Leise (1997: 192) conclude that the "apical ganglion appears to be the only significant neural structure that is lost during prosobranch metamorphosis." A similar conclusion is reached for the loss of the apical organ during metamorphosis in the polyplacophoran Mopalia muscosa (Friedrich et al., 2002). In addition, a recent study of aplacophoran development by Okusu (2002) indicates that the cerebral ganglia develop from ectodermal depressions that are located some distance away from the apical organ. These findings may indicate that the role of the apical organ is strictly limited to the larval phase, without contributing significantly to the adult nervous system. This invites a reconsideration of the supposedly distinct relationship between apical organs and brains in the deuterostomes and protostomes, with only the former being characterized by a complete separation of larval and adult nervous systems in all taxa. A proper understanding of the relationship between the apical organ and adult brain in the deuterostomes is complicated considerably by taxa such as the echinoderms and enteropneusts (Dautov \& Nezlin, 1992; Hay-Schmidt, 2000; Nezlin, 2000; Beer et al., 2001), which despite the possession of well-defined apical organs never develop well-defined cerebral ganglia as adults. One would expect this to be reflected in the scoring of the echinoderms and enteropneusts, for which N44, NI45, S53, $\mathrm{Z145}$, and ZI45 are thus inapplicable. Instead they are scored the same way as taxa that do possess larval apical organs and adult cerebral ganglia which develop independently, e.g., Phoronida. Recent data on the expression of various developmental genes in the apical organs of polychaete and molluscan trochophore larvae and enteropneust tornariae, and the role of their presumed homologs in the development of adult brains in chordates and insects are difficult to interpret at this time. However, they offer further tantalizing clues for the resolution of 
this fascinating issue (e.g. Tagawa et al., 2000; Arendt et al., 2001; Lespinet et al., 2002; Nederbragt et al., 2002). In conclusion, we are nowhere near to a proper understanding of the potential phylogenetic significance of these characters.

\section{Serial repetition of nerve collaterals}

This character is defined in Eernisse et al. (1992) as "ladder-like nervous system with ventrolateral nerve cords and lateral connectives." To avoid confusion, it should be noted that longitudinal nerve cords are typically called connectives and the connections between them commissures instead of lateral connectives.

\section{Character coding}

Z236; E53: serial repetition of nerve collaterals $\mathrm{a} / \mathrm{p}$

Table 21. Scoring conflicts of serially repeated nerve collaterals.

\begin{tabular}{lll}
\hline & polymorphic & $?$ \\
\hline Rhabditophora & E53 & Z236 \\
\hline
\end{tabular}

\section{Character scoring and phylogenetic significance}

Eernisse et al. (1992) introduced this character into metazoan cladistics, and the subsequent analysis of Zrzavý et al. (1998) took over the character with virtually identical scoring, except for Rhabditophora. Although platyhelminths exhibit a substantial amount of variation in the organization of the central nervous system, the homology of the orthogonal organization of the rhabditophoran nervous system appears to be generally agreed upon (Reisinger, 1972; Reuter et al., 1998; Reuter \& Halton, 2001).

According to the character coding all taxa that possess at least two longitudinal connectives that form serially repeated commissural connections should be scored as present. That, however, does not explain the observed character scoring. For example, both Priapulida and Pogonophora are scored as having serially repeated nerve collaterals. Yet, Priapulida possess unpaired ventral nerve cords, and Pogonophora possess only small paired regions in their ventral nerve cords but apparently without serially repeated commissures (Bullock, 1965d; Gardiner \& Jones, 1993; Southward, 1993; Westheide, 1996). Also, when Kinorhyncha are scored as present for this character, then there is no reason why, for example, Nematoda should not be scored as possessing it as well. The nematodes possess similar (but asymmetric) commissures between the longitudinal nerve cords just as the kinorhynchs (Bullock, 1965c; Wright, 1991; Nebelsick, 1993).

A complicating factor for accurately coding and scoring this character is the uncertainty about the homology of the longitudinal nerve cords in different taxa, and consequently, about the homology of the commissures between them. A certain degree of convergent evolution seems likely, for example, within Platyhelminthes where particular commissural patterns within various subtaxa are thought to have evolved independently (Reuter \& Gustafsson, 1995; Reuter et al., 1998; Reuter \& Halton, 2001). Also, the scored taxa may exhibit strikingly different organizations, even in arguably closely related phyla, for example the tardigrades and onychophorans, with the tardigrades showing only four segmental ganglia as the only 'commissures' between the paired ventral nerve cords, and the onychophorans which possess numerous commissures between two ventral cords that lack clearly demarcated ganglia (Schürmann, 1995).

Eernisse et al. (1992) and Zrzavý et al. (1998) code separate characters for an orthogonal nervous system (E103, Z245), and because regularly repeated transverse commissures are an integral part of an orthogon, the taxa scored for these characters should also be scored for E53 and Z236. Nevertheless, this is not the case, indicating that the scoring for both characters could be improved by careful restudy.

\section{Orthogonal nervous system}

The orthogonal nervous is a system with a number of longitudinal nerve cords that are regularly interconnected by transverse commissures.

\section{Character coding}

M20; E103; Z245; ZI48; ZII97: orthogonal nervous system $a / p$ 
H14: orthogonal nervous system a/p/kamptozoan (entoproct) condition

Rather than tabulating and attempting to resolve the many scoring conflicts for this character among the different studies, I think that metazoan cladistics is better served by elucidating the confusion about the definition of this character. Meglitsch \& Schram (1991) defined the orthogonal nervous system as an anterior nerve ring and several longitudinal cords (M20). In contrast Eernisse et al. (1992) defined it as a dense diffuse neural plexus with short peripheral connections and very long interganglionic connections (E103). Not surprisingly, M20 and E103 are scored for very different phyla. More importantly, the phyla that are scored as possessing an orthogonal nervous system for either M20 or E103 do not show any obvious unique similarities. For example, various protostome phyla such as the molluscs and onychophorans have nervous systems answering the definition of M20, but they are not scored accordingly. Similarly, Eernisse et al. (1992) scored an orthogonal nervous system as being present in phyla such as the echinoderms, molluscs, and nematodes, but not in the onychophorans and annelids. This scoring is scarcely based on a proper comparative study of nervous system morphology.

In contrast, the definition of an orthogonal nervous system in later studies (Haszprunar, 1996a; Zrzavý et al., 1998, 2001; Zrzavý, 2003) can be traced back to the work of E. Reisinger. Reisinger (1972: 1) defined the primitive form of an orthogonal nervous system as "a system of multiple, initially equivalent longitudinal nerve cords with, often pseudometamerically arranged commissures" (my translation). Although Reisinger envisaged the orthogonal nervous system to be plesiomorphic for the gastroneuralians, he recognized that a typical orthogon is only present in a few taxa, such as certain platyhelminths. He hypothesized that subsequent evolutionary changes to the nervous system may erase all traces of the original orthogonal nervous system. Reisinger envisaged evolutionary modifications to follow two main lines: reduction of the number of longitudinal nerve cords, and increasing ganglionization of the nerve cords.

Zrzavý et al. (2001) and Zrzavý (2003) score an orthogonal nervous system as present in all non- sessile protostome protostome phyla, including the scalidophorans (Priapulida, Loricifera, Kinorhyncha), and Nematoida (Nematoda and Nematomorpha). Micrognathozoa and Cycliophora are scored as "?". However, when we take into account the disparate nervous system morphologies in the positively scored phyla, we could score an orthogonal nervous system as present in the latter two phyla as well. More importantly, comparative study of nervous system morphology makes clear that an orthogonal nervous system is certainly not present in all these phyla. For example, the tardigrades have paired ventral nerve cords, but without regular commissures. The only connections between the longitudinal cords are the segmental ganglia. Similarly, the gnathostomulids have paired longitudinal ventrolateral nerve cords, but regular commissures are absent (Lammert, 1991; Sterrer, 1996). Even if we broaden the definition by removing the requirement of regular transverse commissures between the longitudinal connectives, we cannot defend the scorings of Z245, ZI48, and ZII97. For example, the priapulids, nematomorphs, and nematodes possess unpaired ventral nerve cords (Storch, 1991; Schmidt-Rhaesa, 1997/1998; Nielsen, 2001; A. Schmidt-Rhaesa, personal communication). These scorings are not based on observations. They can only be defended by the a priori assumption that an orthogonal nervous system is a plesiomorphy for the gastroneuralians, and that this would imply that a failure to observe an orthogonal nervous system indicates a secondary absence. Rather than basing character scoring on an unsupported evolutipnary assumption, a cladistic analysis should aim at testing the assumption. In conclusion, current cladistic characters coding for an orthogonal nervous system have not contributed useful information to the phylogenetic analyses.

\section{$\underline{\text { Statocysts }}$}

The statocysts are sense organs that function as receptors of gravity, directional acceleration, or vibration, and they are widely distributed throughout the animal kingdom. They are usually rounded chambers containing a heavy inclusion called otolith or statolith, and lined by sensory cells. There is considerable variation in the organization of statocysts 
between, or even within, different phyla including the absence or presence of special sensory cells, ciliation of the receptor cells, association with nervous elements, location in the body (associated with the brain, intraepidermal, cuticular), source and chemical composition of the statoliths (endogenous products of various kinds or extraneous elements such as sand grains), fixed or free statoliths, simple or compound statoliths, and the number of statoliths. Their distribution within individual phyla is frequently patchy making it difficult to infer their evolutionary origins and to verify their presence in the ground patterns of phyla. A rigorous comparative study is necessary to elucidate the complex evolutionary history of statocysts throughout the Metazoa.

\section{Character coding}

Wa17; Me60: statocysts a/p

Z252: number of statoliths one to few/10-40

Z253: number of statocyst parietal cells few/many

Table 22. Scoring conflicts for statocysts.

\begin{tabular}{|c|c|c|c|}
\hline & Absent & Present $^{1}$ & $\begin{array}{l}\text { Proposed } \\
\text { scoring }\end{array}$ \\
\hline Syndermata & Wa17 & $\begin{array}{l}\text { Zrzavý et al. } \\
\text { (1998); Me60 } \\
\text { (Rotifera) }\end{array}$ & Absent \\
\hline \multirow{4}{*}{$\begin{array}{l}\text { Nematoida } \\
\text { Scalidophora } \\
\text { Gastrotricha } \\
\text { Gnathosto- } \\
\text { mulida }\end{array}$} & Wa17 & Zrzavý et al. (1998) & Absent \\
\hline & Wa17 & Zrzavý et al. (1998) & Absent \\
\hline & Wa17 & Zrzavý et al. (1998) & Absent or? \\
\hline & Wal7 & $\begin{array}{l}\text { Zrzavý et al. (1998); } \\
\text { Me60 }\end{array}$ & Absent \\
\hline
\end{tabular}

\footnotetext{
${ }^{1}$ Scoring for Zrzavý et al. (1998) is deduced from the scoring of $\mathrm{Z} 252$ and $\mathrm{Z} 253$; positive scoring for these characters presupposes the presence of statocysts.
}

\section{Character scoring and phylogenetic significance}

Wallace et al. (1996) introduced a character coding for the presence of statocysts into a phylogenetic analysis of the aschelminth phyla, and their analysis suggested that the secondary loss of statocysts is an unambiguous synapomorphy of (Gnathostomulida Gastrotricha Syndermata Nematoida Scalidophora). Subsequently, two new cladistic analyses (Melone et al., 1998; Zrzavý et al., 1998) introduced statocyst characters into an analysis of the gnathiferans and the entire Metazoa, respectively. However, these latter analyses introduced considerable character scoring problems.

Although neither the analysis of Wallace et al. (1996), nor that of Melone et al. (1998) fulfilled the domain of definition for statocysts, the problems of character scoring introduced in the analyses of Melone et al. (1998) and Zrzavý et al. (1998) seriously compromise the results. Available information resolves the recorded scoring conflicts in favor of the analysis by Wallace et al. (1996) versus the analyses of Melone et al. (1998) and Zrzavý et al. (1998). No convincing report of statocysts in syndermates exists in the literature. The acanthocephalans definitely lack statocysts (Bullock, 1965c; Dunagan \& Miller, 1991), whereas early reports of rotiferan statocysts are likely erroneous (Bullock, 1965c); and more recent studies do not describe any statocysts (Clément \& Wurdak, 1991; Lorenzen, 1996c). Similarly, statocysts are not reported for the nematoidans, scalidophorans, or gnathostomulids (Bullock, 1965c; Bresciani, 1991; Kristensen, 1991a; Kristensen \& Higgins, 1991; Storch, 1991; Wright, 1991), whereas the presence of unicellular statocysts in the gastrotrichs is equivocal (Bullock, 1965c) and not confirmed in recent studies (Ruppert, 1991b; Lorenzen, 1996e). Consequently, the presence of statocysts is not a valid synapomorphy of Gnathifera as suggested by the analysis of Melone et al. (1998).

Zrzavý et al. (1998) included two characters (Z252, Z253) that code for specializations of statocysts, although a character coding for absence or presence of statocysts was not included in that analysis. Additionally, Z250 coded for the presence of an endon, which is defined as a median cerebral ganglion and adjacent aboral statocyst. The scoring of Z250 for taxa such as the acoelomorphs, gnathostomulids and polychaetes is a problem. The gnathostomulids lack statocysts, and therefore also an endon. The unique organization of the anterior portion of the acoelomorph nervous system indicates that a true cerebral ganglion is lacking (Raikova et al., 1998; 2001), and therefore also the endon. The scoring of the polychaetes is ambiguous because of serious uncertainty about the ground pat- 
tern. Statocysts are only known in a few burrowing and tubicolous families that are spread throughout the Polychaeta, and they were unknown in Oligochaeta (Verger-Bocquet, 1992; Jamieson, 1992; Rouse \& Fauchald, 1997) until the recent study by Locke (2000) showed that the enchytraeid oligochaetes of the genus Grania possess statocysts. The patchy distribution of statocysts within phyla creates uncertainty about the ground patterns for various phyla, and the unambiguous scoring of Z252 and Z253 for several taxa should be reconsidered accordingly. For example, cnidarian statocysts have only been demonstrated in the medusae of medusozoans, being absent in medusozoan polyps and anthozoans, which is in agreement with their evolution within this phylum (Bullock, 1965b; Bridge et al., 1995; Grimmelikhuijzen \& Westfall, 1995; Schäfer, 1996). For the nemerteans, statocysts have only been reported for two interstitial genera of hoplonemerteans (Bullock, 1965a; Turbeville, 1991, 1996). For the echinoderms, statocysts are only known from several holothuroids, and they do probably not belong in the ground pattern of Holothuroidea (Goldschmid, 1996a; Ehlers, 1997). Sometimes statocysts are reported for the echinoids (Ruppert \& Barnes, 1994), but these presumed ambulacral sensory organs are usually called sphaeridia, and neither their anatomical position, nor their structure indicate homology to the statocysts known from holothuroids (Cavey \& Märkel, 1994; Ehlers, 1997). These considerations make a certain degree of convergent evolution of statocysts very likely. Finally, the scorings for many phyla in Zrzavý et al. (1998) have to be adjusted. Statocysts are unknown in Cycliophora (Funch, 1996; Funch \& Kristensen, 1997), Phoronida (Hyman, 1959; Bullock, 1965d; Herrmann, 1996), Chaetognatha (Shinn, 1997), Cephalochordata (Ruppert, 1997), and the aschelminth phyla as discussed above. Furthermore, for those taxa that may possess statocysts in their ground pattern problematic scorings remain. Although the ctenophores and brachiopods are scored to possess one to a few statoliths, the compound ctenophore statolith is made up of 100 smaller statoliths (Hernandez-Nicaise, 1991), while 40 statoliths have been reported for the inarticulate brachiopods (James, 1997).

In conclusion, the scoring of statocyst charac- ters across the Metazoa needs to be carefully reassessed taken the above comments into consideration. So far, the phylogenetic significance of statocysts and associated characters remains unknown.

\section{Nephridial characters}

\section{Protonephridia}

Protonephridia are widespread in the Metazoa, and although variations in morphology are manifested in different ways (see following characters) protonephridia are clearly definable as ciliated tubes that open through the epidermis through a nephropore, but that are proximally closed by one or more terminal cells (cyrtocytes). The distal part of the terminal cell forms a hollow cylinder typically perforated by clefts, and which serves to support an extracellular filtration membrane. The terminal cells are joined to canal cells and nephropore cells, although the exact configuration varies between taxa. Wilson \& Webster (1974), Ruppert \& Smith (1988), and Bartolomaeus \& Ax (1992) provide useful reviews of protonephridial structure and function throughout the Metazoa. Ruthensteiner et al. (2001) found that the protonephridia of larval and juvenile scaphopods are composed of only two cells, which contradicts the universality of the tripartite structure (terminal cells, canal cells, and nephropore cells) of protonephridia proposed by Bartolomaeus \& Ax (1992). Although Nielsen $(1995,2001)$ restricts the definition of protonephridia to ectodermally derived canals, his scoring for NI54 in the gladistic data matrix does not exhibit this restriction. Taxa where mesoderm has been implicated to contribute to the formation of the protonephridia (either as the sole source or as a partial contributor), such as the molluscs and annelids (Verdonk \& Biggelaar, 1983; Bartolomaeus, 1999), are scored as well. The paucity of reliable information on the germ layer source of protonephridia leads me to conclude that the application of a structural homology criterion without consideration of the embryonic source is currently the best diagnosis for protonephridia across the Metazoa (see Ruppert, 1994 for an example of varying contributions of mesoderm and ectoderm to the homologous nephridia in larval enteropneust and asteroids). The 
Table 23. Scoring conflicts for protonephridia.

\begin{tabular}{|c|c|c|c|c|c|}
\hline & $\begin{array}{cc}\text { Absent } & \\
& , \ldots\end{array}$ & Present & $?$ & Polymorphic & $\begin{array}{l}\text { Proposed } \\
\text { scoring }\end{array}$ \\
\hline Phoronida & M31; E99 & $\begin{array}{l}\text { Z48; ZI29; N54; } \\
\text { NI54; S63; ZII66 }\end{array}$ & & & Present \\
\hline Loricifera & & $\begin{array}{l}\text { Z48; N54; NI54; } \\
\text { S63; Wa40; ZII66 }\end{array}$ & M31 & & Present \\
\hline Nematoda & $\begin{array}{l}\text { Z48; ZI29; N54; } \\
\text { NI54; S63; M31; E99; } \\
\text { ZII66 }\end{array}$ & L56 & & & Absent \\
\hline Annelida & M31; E99; RI7 & $\begin{array}{l}\text { N54; NI54; S63; Z48; } \\
\text { ZI29; H20; ZII66 }\end{array}$ & & $\begin{array}{l}\text { Wa40 (for } \\
\text { polychaetes) }\end{array}$ & Present \\
\hline Pogonophora & & RI7; E99; M31 & $\mathrm{Z} 48$ & & $?$ \\
\hline Echiura & M31; E99; RI7 & $\begin{array}{l}\text { Z48; ZI29; S63; } \\
\text { R139; H20; ZII66 }\end{array}$ & & & Present \\
\hline Sipuncula & $\begin{array}{l}\text { N54; NI54; M31; } \\
\text { S63; ZI29; RI7; R139; } \\
\text { E99; ZII66 }\end{array}$ & & $\mathrm{H} 20 ; \mathrm{Z} 48$ & & Absent \\
\hline Mollusca & RI7; M31; E99 & $\begin{array}{l}\text { H20; N54; NI54; } \\
\text { S63; Z48; R139; } \\
\text { ZI29; ZII66 }\end{array}$ & $\cdot$ & . & Present \\
\hline Nemertea & E99 & $\begin{array}{l}\text { H20; N54; NI54; } \\
\text { S63; Z48; ZI29; } \\
\text { M31; ZII66 }\end{array}$ & R139 & & Present \\
\hline Brachiopoda & $\begin{array}{l}\text { ZI29; E99, M31; N54; } \\
\text { NI54; S63; ZII66 }\end{array}$ & 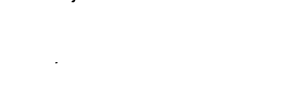 & $\mathrm{Z} 48$ & & Absent \\
\hline
\end{tabular}

significance of heterotopic shifts in the origin of protonephridia remains to be studied, and the potential role of heterochrony may illuminate the relationships between larval and adult protonephridia in several taxa, such as the polychaetes.

\section{Character coding}

H20; HallIb; N54; NI54; S63; Z48; ZI29; ZII66; R139; L56; Wa40; Es6; E99 (emphasis on multiciliated cells): protonephridia $\mathrm{a} / \mathrm{p}$

Eh6: loss of protonephridia $a / p$

M31: no special excretory organ/protonephridia

RI7: protonephridia/metanephridia/pericardioducts

\section{Character scoring and phylogenetic significance}

A number of these scoring conflicts can be reduced to different decisions about the choice of comparable semaphoronts. Rouse \& Fauchald (1995) scored their characters according to adult morphology, and while explicit mention is lacking, the scor- ing of protonephridia in Meglitsch \& Schram (1991) and Eernisse et al. (1992) is also consistent with an exclusive scoring of adults. This would explain their character scoring for the phoronids, annelids, echiurans, and molluscs (all scored as lacking protonephridia). In these taxa, protonephridia are likely to be present in the larval ground pattern (Heimler, 1988; Smith \& Ruppert, 1988; Bartolomaeus, 1989a, b, 1993b, 1995, 1998, 1999; Zimmer, 1991, 1997; Hermann, 1997; Rouse, 1999; Haszprunar \& Ruthensteiner, 2000; Ruthensteiner et al., 2001), while adult protonephridia are entirely lacking (phoronids), or restricted to isolated cases not likely to be representative for the phyla's ground patterns (dwarf male Bonellia in Echiura; adult paedomorphic Rhodope in Mollusca; various polychaete annelids; Westheide, 1986; Bartolomaeus, 1989c; Schuchert, 1990; Haszprunar, 1996d, 1997; Rouse, 1999; Rouse \& Fauchald, 1997). Adoption of a structural homology criterion necessitates that all semaphoronts are taken into account during character scoring. 
Loricifera possess protonephridia (Kristensen, 1991a), as opposed to M31 (the chapter on loriciferans in Meglitsch \& Schram, 1991 does report protonephridia as contrast to the scoring in their data matrix).

Nematodes lack protonephridia (Wright, 1991), contra L56.

Protonephridia have been reported for both pogonophore larvae as well as adults (reviewed in Rouse \& Fauchald, 1995; 1997; Salvini-Plawen, 2000 ), but not in adult vestimentiferans (Schulze, 2001). Pending a comparative ultrastructural analysis of nephridia in different pogonophores, the ground pattern state may be scored as uncertain (Z48) (Southward, 1993; Rouse \& Fauchald, 1995).

Protonephridia have never been reported for sipunculans, neither for adults nor for larvae. Because protonephridia are typically present in the trochophore larvae of other spiralian phyla, this may lead to a scoring of a '?' as for H20 and Z48. However, because we cannot predict whether future studies will identify sipunculan protonephridia, and because their absence is an accurate representation of our current knowledge, it is better justified to score 'absence of protonephridia' as is done in the remaining cladistic analyses.

The scoring of E99 for the nemerteans is obviously erroneous. Nemerteans evidently do have protonephridia in the adults, and reports for larvae exist as well (Bartolomaeus, 1985; Turbeville, 1991). The scoring of a '?' for the nemerteans for R139 can also be changed to 'presence of larval protonephridia.' Rouse (1999) considers them unknown because "they appear late and do not really function in larvae per se." However, he reports their demonstration in pilidium larvae, and Bartolomaeus (1985) reported their presence in a Desor's larva (generally regarded as a modified lecithotrophic pilidium larva). This would make the evidence for scoring larval protonephridia present in the nemerteans just as strong as that which justifies Rouse's scoring of larval protonephridia for the platyhelminths (based on their demonstration in Müller's larvae: Ruppert, 1978; see Rohde, 2001, figs. 19.1 and 19.8 for illustrations of protonephridia in a Götte's larva). However, considering that the presence of polyclad and pilidium larvae in the ground patterns of the platyhelminths and nemerteans is highly unlikely (see above), these findings are not relevant for the scoring of larval protonephridia in these phyla.

Protonephridia have never been observed during any stage of the brachiopod life cycle, and this warrants their scoring as 'absent' contra Z48.

E99 defined protonephridia as being characterized by multiciliated cells. However, this does not explain the adopted scoring. For example, the nemerteans typically have protonephridia with multiciliated cells, but were scored as lacking these, whereas gnathostomulid protonephridia possess only monociliated cells (the terminal cell), but they were scored as having multiciliated protonephridial cells (Lammert, 1991; Bartolomaeus \& Ax, 1992). Furthermore, considering the variation in protonephridial designs throughout the Metazoa, including the existence of non-ciliated, mono-, bi- or multiciliated cells, and the observation that taxa such as the polychaetes and nemerteans may change the number of cilia per cell during ontogeny indicate that E99 adopts an unnecessarily restricted definition. Furthermore, it is essential to specify which protonephridial cells are multiciliated, because ciliation of terminal cells, duct cells, and nephropore cells may vary independently between taxa.

Littlewood et al. (1999a) infused the scoring of their character L56 with an unwarranted a priori assumption about the direction of evolutionary change for the acoelomorph platyhelminths. These were scored as ' $0 / 1$ ' instead of ' 0 ' because their phylogenetic placement in previous studies would suggest that they may have lost protonephridia, rather than that these were primitively absent. However, several recent molecular and morphological phylogenetic analyses have suggested that the acoels and also the nemertodermatids may be the basalmost extant bilaterians, and may consequently have never possessed protonephridia (Haszprunar, 1996b; Ruiz-Trillo et al., 1999, 2002; Baguñà et al., 2001; Jondelius et al., 2002; Telford et al., in press). Littlewood et al.'s a priori assumption about the evolution of protonephridia in the acoelomorphs reflected in the character coding can only lead to circular reasoning.

Note that Rouse (1999) accidentally recorded the scoring for character R139 (protonephridia) in the 
wrong column of his Appendix 4, that of character $\mathrm{R} 138$.

All cladistic studies considered here, with the exception of Haszprunar (1996a, b), surprisingly suggest that protonephridia are homoplastic within Bilateria, and have evolved not at the base, but within the Bilateria, usually at the base of a large protostome clade. Instead, Haszprunar (1996a, b) suggest that protonephridia are a unique synapomorphy of all bilaterians except Acoelomorpha. However, for a proper interpretation of Haszprunar's analyses, one should take into account that his taxon selection did not fulfill the domain of definition for protonephridia. Interestingly, Nielsen et al. (1996), Nielsen (2001), Zrzavý et al. (1998, 2001), and Sørensen et al. (2000) indicate that phoronid actinotroch larvae have independently evolved protonephridia. Furthermore, the analyses of Meglitsch \& Schram (1991), and Zrzavý et al. (1998, 2001) indicate that the protonephridia of scalidophorans may have evolved convergently with respect to those of the other protostomes. Finally, multiple losses of protonephridia are indicated for taxa such as Panarthropoda, Nematoida, and Acoelomorpha (Nielsen et al., 1996; Nielsen, 2001; Sørensen et al., 2000; Zrzavý et al., 1998, 2001).

\section{Protonephridia with channel cell completely sur-} rounding lumen

This character refers to protonephridia in which the lumen is located intracellularly in the canal cells. This contrasts with the situation in which the canal cells are folded around the protonephridial lumen, which can be recognized by the presence of intercellular junctions that close off the extracellular lumen. Unfortunately, some terminological confusion is present in the literature. Some authors restrict the term "percellular" lumen to instances of a truly intracellular lumen (Ahlrichs, 1995), whereas other authors apply this term also to instances in which the protonephridial canal cell is folded around the extracellular lumen as long as it enwraps the cilia and microvilli in the lumen (Bartolomaeus, 1993b, 1995).

\section{Character coding}

A15; HaVIIIc; P68; Z52: protonephridia with channel cell completely surrounding lumen $a / p$

\section{Character scoring and phylogenetic significance}

This character is uniformly scored for Gnathostomulida and Syndermata, but insufficient information exists on the ultrastructure of acanthocephalan protonephridia to allow the unambiguous determination of their canal morphology. Consequently, Z52 needs to be rescored as '?' for Acanthocephala. When assessing the phylogenetic significance of intracellular canal lumens in rotifer and gnathostomulid protonephridia, it should be kept in mind that those of the former are mainly syncytial (although the cellular canal components also appear to have an intracellular lumen), whereas those of the latter are cellulä (Ahlrichs, 1993a, b). Although not scored in the studies that included this character, further instances of true intracellular protonephridial ducts have been reported for various other phyla, including the platyhelminths, gastrotrichs, and polychaetes (Smith \& Ruppert, 1988; Rieger et al., 1991b; Ruppert, 1991b). Nevertheless, the scattered distribution of these features causes doubt about their phylogenetic significance. The computer-assisted cladistic analyses of Zrzavý et al. (1998) and Peterson \& Eernisse (2001) suggest convergence of this trait in the rotifers and gnathostomulids.

\section{Podocytes/terminal cells/nephrocytes}

\section{Character coding}

P67: podocytes/terminal cells/nephrocytes a/p/excretory organ of apomorphic design with cells without any obvious similarity to podocytes H21; Z43: podocytes a/p

\section{Character scoring and phylogenetic significance}

Haszprunar (1996a) and Zrzavý et al. (1998) both scored podocytes for Nemertea and Neotrochozoa, while the latter study additionally scored them present for Phoronida and Enteropneusta. As noted 
in Jenner (2001a), a variety of misscorings for Z43 could be identified, including the onychophorans, arthropods, echinoderms, pterobranchs, and vertebrates, which were all incorrectly scored as lacking podocytes (Ruppert \& Smith, 1988; Storch and Ruhberg, 1993; Hessler and Elofsson, 1995; Nielsen, 1995; Benito and Pardos, 1997). Note that the scoring of a '?' for podocytes in the pogonophorans in Zrzavý et al. (1998) can be changed to ' 0 ' since a recent study failed to find support for their presence (Schulze, 2001). Similarly, the scoring of podocytes for pogonophorans and brachiopods in table 4 of Haszprunar (1996d) is unsupported by data. The true phylogenetic significance of the podocytes will only emerge after this character is correctly scored across the Metazoa.

Peterson \& Eernisse (2001) did not code a character solely for podocytes, but instead $\mathrm{P} 67$ proposes a primary homology of different cell types that are all thought to be part of filtration nephridia (the podocytes and nephrocytes are components of metanephridial systems, but the latter is a term usually restricted to the arthropods and onychophorans, and terminal cells are part of protonephridia). This scoring is supported by data on the ontogenetic continuity of protonephridia and metanephridia in certain polychaetes and phoronids, and by a continuum in cytological differentiation and function between the different cell types involved (Ruppert \& Smith, 1988; Smith \& Ruppert, 1988; Bartolomaeus \& Ax, 1992; Smith, 1992; Ruppert, 1994; Haszprunar, 1996d).

\section{Muscles}

\section{Muscle cells}

Muscle cells contain a system of contractile filamentous actin-myosin. They exist in two distinct varieties (Rieger \& Lombardi, 1987; Bartolomaeus, 1994). First, apolar cells that are completely surrounded by extracellular matrix, and which are called myocytes or muscle cells. Second, cells with a clear apical-basal polarity, basally resting on extracellular matrix (a basal lamina), and with the apical end facing an inner body space.

\section{Character coding}

M6: no special muscle cells/well-developed muscle cells (note that coding of Schram (1991) is different and should be rescored accordingly)

P35: endomesodermal muscle cells $a / p$

Z257: muscles a/p

\section{Character scoring and phylogenetic significance}

Muscle cells are universally considered as a eumetazoan autapomorphy, which is in agreement with the analyses of Meglitsch \& Schram (1991) and Zrzavý et al. (1998). However, M6 misscored the platyhelminths, gnathostomulids, and gastrotrichs. These three phyla possess well-defined muscle cells (Rieger et al., 1991b; Lammert, 1991; Ruppert, 1991b). These accidental misscorings may be understood by considering the data matrix of Schram (1991). Although the data matrices of Meglitsch \& Schram (1991) and Schram (1991) are virtually identical, several characters have differing character codings, among them M6. M6 codes as the primitive state "no special muscle cells" while character 6 in Schram (1991) codes "locomotion by action of cilia or flagella." With identical character scorings this results in correct scorings for the three phyla in Schram (1991) but incorrect scorings for M6. The scoring of the poriferans deserves a comment. Porifera is scored as lacking muscle cells in both Meglitsch \& Schram (1991) and Zrzavý et al. (1998), but contractile cells superficially resembling vertebrate smooth muscle cells are present in many of the cellularian sponges (Harrison \& de Vos, 1991). These cells are usually referred to as myocytes, a term typically used for eumetazoan muscle cells. Nevertheless, contractile cells with a functional system of fibrillar actin and myosin are also found in other, unicellular non-metazoan eukaryotes (Bovee, 1991). The probable presence of an actin/myosin system in sponges (Harrison \& De Vos, 1991) is therefore no straightforward argument for the presence of true muscle cells or myocytes, and accordingly special homology of contractile sponge cells with eumetazoan muscle cells is not accepted here. Trichoplax also possesses contractile cells (fiber cells) (Grell \& Ruthmann, 1991), and these may perform both muscle and nerve cell functions. 
Although M6 scores the mesozoans as entirely lacking muscle cells, contractile cells have been demonstrated in the orthonectids (Ax, 1995; Haszprunar, 1996c), and these may be muscle cells as is reflected in the scoring of Z257.

Endomesodermal muscle cells are considered an autapomorphy of Acrosomata by Peterson \& Eernisse (2001) in agreement with other recent works (e.g. Martindale \& Henry, 1998). However, many taxa were not scored for P35, presumably because cell lineage data is not present to confirm the exact source of the mesoderm. Interestingly, strict application of the definition of endomesoderm (see also under Secondary body cavity, coelom; ontogenetic source) would necessitate the rescoring of several taxa that were unambiguously scored as only possessing endomesoderm. As an example let us consider Nematoda. For Caenorhabditis elegans the entire cell lineage is known, which should in principle allow us to make a definite conclusion on the source of mesoderm. Strikingly, the cell lineage of C. elegans clearly shows that all mesoderm is derived from cells that only form ectoderm and mesoderm (e.g. Table 37.1 in Nielsen, 2001), while the nematode endodermal cell lineage is already completely separated from the sources of other organs at the eight cell stage (Voronov et al., 1998; Voronov, 1999). This leads to the inescapable conclusion that this worm only possesses ectomesoderm! Similarly, although the resolution of the cell lineage of the gastrotrichs is limited, it nevertheless shows that all mesoderm derives from precursor cells that will only produce ectoderm and mesoderm (Table 35.1 in Nielsen, 2001), and thus represent ectomesoderm. Similar indications for the presence of ectomesodermal muscles are reported for the rotifers (Nielsen, 2001: 300). Freeman \& Martindale (2002) recently showed that part of the mesoderm in the phoronids also derives from ectoderm, and these authors suspect that the same holds true for the brachiopods as well, and so, modern cell lineage tracing techniques hold great promise when applied to the many poorly studied phyla (see Martindale \& Henry, 1999 and Henry et al., 2000 for nice examples of recent advances in our understanding of mesoderm sources in the ctenophores and acoels). Obviously, we also need to sample multiple species within phyla to assess character variability, but these considerations at least show that the widely adopted interpretation of endomesoderm as a acrosomatan or bilaterian autapomorphy deserves closer scrutiny.

\section{Digestive system}

\section{$\underline{\text { Intestinal cell ciliation }}$}

To create an accurate character definition, it should be clearly specified what part of the gut is scored for ciliation. Many phyla possess cuticularized foreand hindguts from which cilia may be lacking, while at the same time the digestive midgut region without a cuticle can be ciliated. It is therefore important to distinguish pharynx, esophagus, stomach, intestine, and rectum. I assume the coding of "gut" in P93, ZII49, and Wa22 to refer to the intestine.

\section{Character coding}

Z225: intestinal cells ciliated/non-ciliated

ZII49: non-ciliated gut $\mathrm{a} / \mathrm{p}$

P93: digestive gut without cilia $a / p$

Wa22: ciliated gut $\mathrm{a} / \mathrm{p}$

\section{Character scoring and phylogenetic significance}

The chaetognaths possess ciliated absorptive and glandular intestinal cells (Shinn, 1997), justifying a rescoring for $Z 225$.

Although the nematomorphs possess a reduced digestive tract that some authors claim is not involved in the uptake of nutrients (explaining the scoring of Wa22 and Z225), the nematomorphs nevertheless do possess some genuine intestinal cells adorned with microvilli, but lacking cilia (Bresciani, 1991; Schmidt-Rhaesa, 1996). This is in accordance with the scoring of P93 and ZII49.

Most of the gastrotrichs lack intestinal cilia, but the several species in which ciliated intestinal cells do occur may be phylogenetically the most basal macrodasyoids (Hochberg \& Litvaitis, 2000, 2001a). The resulting uncertainty about the gastrotrich ground pattern is therefore best reflected in the scoring of Wa22.

When an intestine is present in rotifers, its lining cells are abundantly ciliated (Clément \& Wurdak, 
Table 24. Scoring conflicts for intestinal ciliation.

\begin{tabular}{|c|c|c|c|c|c|}
\hline & Absent & Present & $?$ & Polymorphic & $\begin{array}{l}\text { Proposed } \\
\text { scoring }\end{array}$ \\
\hline Chaetognatha & & P93; ZII49 & $\mathrm{Z} 225$ & & Present \\
\hline Nematomorpha & P93; ZII49 & & Wa22; Z225 & & Absent \\
\hline Gastrotricha & P93; ZII49 (Chaetonotida) & Z225; ZII49 (Macrodasyida) & & $\mathrm{Wa} 22$ & $?$ \\
\hline Rotifera & & P93; Z225; Z1149 & & $\mathrm{Wa} 22$ & Present \\
\hline Acoelomorpha & P93; ZII49 (Acoela) & Z225; Wa22 (Turbellaria) & $\begin{array}{l}\text { ZII49 (Nemer- } \\
\text { toderma-tida) }\end{array}$ & & Absent \\
\hline Rhabditophora & P93 & $\begin{array}{l}\text { Z225; Wa22 (Turbellaria); } \\
\text { ZII49 }\end{array}$ & & & $?$ \\
\hline Polychaeta & & Z225; P93 (Annelida); ZII49 & & $\mathrm{Wa} 22$ & Present \\
\hline Nemertea & P93 & Z225; ZII449 & & & Present \\
\hline
\end{tabular}

1991), in agreement with the scoring of P93 and $\mathrm{Z} 225$.

The acoel platyhelminths appear to lack intestinal cilia, while earlier reports of intestinal cilia in the nemertodermatids (Rieger et al., 1991b) so far have not been confirmed. A recent cladistic analysis of the Nemertodermatida (Lundin \& Sterrer, 2001) showed that the nemertodermatids primitively lack intestinal cilia. Ciliated intestinal cells do occur in the catenulids, macrostomids, and various other rhabditophorans, but the majority of the turbellarians is reported to lack intestinal cilia (Rieger et al., 1991b). Consequently, the rhabditophoran ground pattern remains at this time uncertain.

Although there may be polychaetes that lack intestinal cilia, the sources available to me suggest their unambiguous presence throughout the annelids, in agreement with the scoring of P93 and Z225 (Michel, 1988; Saulnier-Michel, 1992; Jamieson, 1992; Fernández et al., 1992).

The nemertean intestine is ciliated (Turbeville, 1991), in agreement with the scoring of Z225. However, an unpublished revised version of the data set of Peterson \& Eernisse (2001) (K. J. Peterson, pers. comm.) corrected the scoring of P93.

Z225 also scored Xenoturbella as having nonciliated intestinal cells. However, in view of the fact that Zrzavý et al. (1998) do not provide an exact source for this information, and because ultrastructural studies of the gastrodermis of Xenoturbella have not yet been performed (Israelsson, 2000 ), scoring of a '?' appears at this time to be the best option.

Z225 and ZII49 score Lobatocerebrum as pos- sessing ciliated gut cells. However, both the esophagus and midgut of Lobatocerebrum are generally non-ciliated. Only the pharynx and the hindgut cells are generally ciliated (Rieger, 1981). However, because sporadic midgut cells may bear cilia the scoring of Z225 and ZII49 has some support.

It should be noted that P93 should be rescored for Fungi, Choanoflagellata, Porifera, and Placozoa since these taxa lack a digestive gut, and are thus 'inapplicable' for this character.

Irrespective of which morphological phylogeny of the Metazoa is consulted, it can be concluded that intestinal cilia have been lost at least a number of times independently, namely in introvertans and panarthropods, in Gnathostomulida and Limnognathia maerski (Micrognathozoa), which also totally lacks intestinal cilia (Kristensen \& Funch, 2000), and possibly in the acoelomorph platyhelminths. The loss of intestinal cilia may thus be a synapomorphy (albeit convergent) of ecdysozoans additional to those discussed by Schmidt-Rhaesa et al. (1998) and Nielsen (2001) (see also Z225 in Zrzavý et al., 1998), albeit a homoplastic one. Moreover, the newly defined clade Gnathifera that includes Micrognathozoa may also be supported by the loss of intestinal cilia, with a subsequent reversal to ciliated intestinal cells in the rotifers. Isolated instances of intestinal cilia occurring in phyla that are otherwise characterized by the lack of intestinal cilia, such as the arthropods and nematodes, perhaps can reasonably be assumed to have convergently evolved within the respective monophyla (Nielsen, 2001). 
Table 25. Scoring conflicts of anus.

\begin{tabular}{lllll}
\hline & Absent , & Present & Polymorphic & $?$ \\
\hline Gnathostomulida & M16; E96; Wa21 & $\begin{array}{l}\text { Z72 (autapo.); H11 } \\
\text { (autapo.); P94; NI32 }\end{array}$ & L54; ZII50 \\
Pogonophora & M16 & RI5 & E96 & \\
Acanthocephala & & M16; L54 & Z72; ZII50 \\
\hline
\end{tabular}

\section{Anus}

A variety of studies included characters that coded for the presence of an anus. Different character codings have been designed to incorporate information on the presence of a hindgut, rectum, or proctodeum.

\section{Character coding}

M16; P94: no anus/anus

RI5: digestive tract with mouth only/anus present (at least transiently)

Z72: anus a/gnathostomulid-like/p

$\mathrm{H} 1 \mathrm{l}$ : anus a/gnathostomulid type/true hindgut

E96: anus with proctodeum (completely unidirectional alimentary canal) $a / p$

Z125: reduction of hindgut/anus system $a / p$

ZII50: hindgut/anus a/p

HaXIa; L54: true hindgut with anal opening $a / p$

$\mathrm{Wa} 21$ : complete gut terminating with a functional anus $\mathrm{a} / \mathrm{p}$

NI32: mouth and anus a/p (misscored platys)

\section{Character scoring and phylogenetic significance}

All comprehensive morphological cladistic analyses support (although not unambiguously in all studies) the evolution of a unidirectional digestive tract with an anus as an autapomorphy for Bilateria (Zrzavý et al., 1998; Giribet et al., 2000; Meglitsch \& Schram, 1991; Nielsen, 2001; Peterson \& Eernisse, 2001). Consequently, the absence of an anus in taxa such as the platyhelminths (misscored for NI32), pogonophorans, articulate brachiopods, and acanthocephalans must be considered secondary. In contrast, other studies suggested the independent evolution of an anus in the protostomes and deuterostomes (Brusca \& Brusca, 1990; Rouse \& Fauchald, 1995; Ax, 1989, 1995; see further dis- cussion under Nemertea). However, these studies only considered a restricted set of metazoan phyla, and consequently, their results do not constitute real tests of the homology of anuses throughout the Metazoa.

The distinctive morphology of the gnathostomulid anal system defies unambiguous interpretation (see discussion under Platyhelminthes for details). Until very recently, the morphology of the terminal end of the gnatkostomulidan digestive tract appeared to be unique among metazoans as is reflected in the character coding of $\mathrm{Z72}$ and H11. However, the anus system found in the newly described Micrognathozoa is very similar in ultrastructural detail (Kristensen \& Funch, 2001). At present, all the adopted character scorings listed in the above table can reasonably be defended, with the exception of L54. L54 included the presence of a "true hindgut" in the character definition. Gnathostomulids unequivocally lack a distinguishable (cuticlelined) hindgut. Inclusion of the presence of a hindgut in the character definition may also necessitate rescoring of gastrotrichs for L54, ZI25, and ZII50 (Ruppert, 1991b). The distribution of hindguts within Gastrotricha (only in paucitubulatinid chaetonotidans) suggests they evolved within the phylum (Hochberg \& Litvaitis, 2000).

The scoring of M16 and E96 for the pogonophorans is misleading. The presence of an anus is well documented for earlier ontogenetic stages in the vestimentiferans (Gardiner \& Jones, 1993: fig. 36A; 1994: fig. 1E; Southward, 1999: fig. 6.9), and an anus has also been reported for at least one species of perviate pogonophore (Flügel \& CallsenCencic, 1992). This is in accordance with our preference for scoring characters across entire life cycles, rather than focusing on arbitrarily chosen semaphoronts (Jenner, 2001a). Furthermore, none of the recently proposed phylogenetic hypotheses for the placement of the pogonophorans has raised doubt 
as to the derived nature of the lack of an anus. The phylogenetic significance of the ontogenetic reduction of the anus in pogonophorans is therefore restricted to being apomorphic for the group.

The acanthocephalans entirely lack a digestive system and M16 and L54 should therefore be rescored (Dunagan \& Miller, 1991).

It should be noted that several phyla that are habitually scored as possessing a digestive tract with an anus, in fact possess a cloaca that functions as a shared opening for the gut and the reproductive system, as is found for instance in nematodes, nematomorphs, and rotifers.

Finally, the character coding for ZI25 knowingly introduces an a priori phylogenetic conclusion (the lack of anus in the platyhelminths as evolutionarily derived), resulting in a character that only serves to unite the catenulids and rhabditophorans on the basis of circular reasoning. Furthermore, although the gnathostomulids may posses an anus, justifying their scoring of a '?,' they should instead be scored as lacking a "true hindgut with anal opening" since the gnathostomulids definitively lack a true hindgut (Lammert, 1991). As argued above, hindguts may have evolved within Gastrotricha.

\section{Miscellaneous characters}

\section{Frontal gland complex}

A broad definition of a frontal gland complex (frontal organ) specifies a collection of various glands (mucous, rhabdite, rhammite), that may or may not be associated with ciliary sensory cells, and that is located at the anterior end of the animal. A frontal organ is a more narrowly defined frontal gland complex that describes the special configuration found in the acoelomorph platyhelminths, in which several independent gland cell necks join in a common apical pore (Smith \& Tyler, 1986; Rieger et al., 1991b; Ehlers, 1992; Littlewood et al., 1999a: L47).

\section{Character coding}

P108: frontal complex a/p

HaIe; Z224; ZI56; ZII105: frontal glandular system $\mathrm{a} / \mathrm{p}$
HaVIIc: loss of frontal glandular complex

H17: frontal gland a/p/entoproct type

HaVIIId: frontal (=praepharyngeal) glands $a / p$

\section{Character scoring and phylogenetic significance}

Zrzavý et al. (1998, 2001) and Zrzavý (2003) score a frontal glandular complex present in the platyhelminths (acoelomorphs and rhabditophorans), entoprocts, Lobatocerebrum, and nemerteans. In contrast, Peterson \& Eernisse (2001) only score it present in the platyhelminths, in agreement with Ehlers (1992) and Ax (1995). A precise definition is the first requisite needed for an accurate estimation of the phylogenetic significance of the frontal glands found in such distantly related taxa. Expectedly, the central feature of all frontal glands is the presence of glandular cells that may synthesize and secrete a variety of products. However, authors may differ with regards to the inclusion of sensory elements in the character definition. For example, with respect to the acoelomorph frontal organ, Smith \& Tyler (1986: 77) conclude that it "is strictly a glandular organ and evidently is not sensory." In contrast, Rieger (1996c: 223-224) writes about "das Frontalorgan, ein in einem speziellen Porus an der Vorderspitze ausmündendes Drüsen- und Sinnesorgan..." To avoid confusion about homology, the precise morphological conformation should be clearly specified. For example, it should be noted that the frontal organ in entoproct larvae is not considered glandular itself, rather glandular cells are reported to surround the frontal organ in loxosothellid entoproct larvae (apparently not in pedicellinids and barentsiids) (Nielsen, 1971, 2001). The frontal organ sensu stricto is regarded as a sensory organ (Nielsen, 1971, 2001; Emschermann, 1996). However, a close association of glandular and sensory cells appears to be common, though not universal, for frontal complexes in the different taxa, e.g., platyhelminths (Ruppert, 1978, Smith \& Tyler, 1986), entoprocts Nielsen $(1971,2001)$, nemerteans (Turbeville, 1991).

The phylogenetic significance of frontal glandular complexes has so far not been resolved. Based on morphological differences between the systems in different phyla, and the presence of similar but apparently convergent glandular systems in other 
taxa such as the polychaetes and bryozoans, Haszprunar (1996a) estimated a low probability of homology. Friedrich et al. (2002) described the development of anterior frontal glands in a chiton, with similarities to frontal glands described in other phyla. Turbeville (1997) has doubts about the homology of the platyhelminth and nemertean frontal complexes. The phylogenetic distribution of Z224 is equivocal about a single evolutionary origin of this character, but ZI56 indicates unambiguous convergence of frontal glands in the rhabditophorans and a clade of Entoprocta + Lobatocerebrum + Nemertea. The analysis by Zrzavý (2003) shows at least unambiguous convergence of the frontal glands in acoelomorphs versus the other bilaterians, while the cladistic analysis of Haszprunar (1996a) suggested unequivocal convergence in Acoelomorpha, Rhabditophora, Entoprocts and probably Lobatocerebrum + Nemertea. Finally, it should be noted that although Zrzavý et al. (1998, 2001) and Zrzavý (2003) included the Cycliophora in their analyses, they did not score the large glands that open at the anterior end of the body of cycliophoran Pandora larvae, chordoid larvae, and the free swimming females (Funch, 1996; Funch \& Kristensen, 1997). Considering the range of morphologies scored present for Z224, ZI56, and ZII105, it would be reasonable to score Cycliophora present for this trait as well. The newly described Micrognathozoa lacks anterior glands (Kristensen \& Funch, 2000).

\section{Locomotion}

\section{Character coding}

Z132: free locomotion $\mathrm{p} / \mathrm{absent}$ in adult Z258: locomotion in adults ciliary/muscular

\section{Character scoring and phylogenetic significance}

Z132 suffers from a major problem, namely the lack of any morphological similarity that would justify homology of the character states in different phyla. Moreover, many character scorings are puzzling. For example, homology is proposed for the inability to locomote freely as an adult in taxa as diverse as the myzostomids, rotifers, echiurans, pterobranchs, ectoprocts, brachiopods, poriferans, and cnidarians. This scoring totally contradicts morphological evidence. For example, individual zooids of the cephalodiscid pterobranchs are freely movable within the common coenecium, and the zooids and their attached adhesive disc may even move out of the coenecium to start building a new one elsewhere in the face of adverse local conditions (Goldschmid, 1996b; Nielsen, 2001). Echiura are active burrowers in soft sediments or inhabitants of rock crevices, and although they are sometimes described as semi-sessile, they are anything but unable to move freely as adults. It is furthermore unexplainable that the enteropneusts, which inhabit similar environments and exhibit comparable habits to the echiurans, are in contrast scored as having free adult locomotion. Although the myzostomids live on, or in, echinoderm hosts, and sessile species do exist, others can certainly move freely about as adults (Grygier, 2000). Finally, scoring of the inability to move freely as adults is rather surprising for medusozoan cnidarians. The rotifers are also typically free living. Homology of the supposed inability to move among these taxa, and in taxa with truly sessile adults, such as the ectoprocts (excepting the rare occurrence of actively moving colonies) and articulate brachiopods, is entirely unsupported. The high degree of homoplasy of this character on the morphological phylogeny of Zravy et al. (1998) is in agreement with the expected lack of any phylogenetic significance of the inability to move as adults across distantly related phyla.

A logical conflict is introduced into the data matrix of Zrzavý et al. (1998) for character Z258 for taxa that are scored as not typically moving as adults. These taxa are scored by default as moving by means of muscular action. This leads to conflicting scorings for taxa such as the cycliophorans, ectoprocts and brachiopods, which are claimed to be non-locomotory as adults, but which are also scored as moving by muscle action. Moreover, various taxa that exhibit adult locomotion are erroneously scored, including Ctenophora (incorrectly scored for muscular locomotion: Hernandez-Nicaise, 1991), monogonont rotifers (scored '?', but they move mainly by ciliary action: Lorenzen, 1996c), and Lobatocerebrum which glides mainly through ciliary action rather than through muscle activity. (Rieger, 1980). However, in several taxa locomotion may be ef- 
fected through the combined action of both cilia and muscles. For example, the exclusive scoring of the molluscs for muscular adult locomotion ignores the importance of muco-ciliary gliding on a ventral foot, which is widely considered as one of the key autapomorphies of the phylum (SalviniPlawen, 1968, 1980a, 1990; Haszprunar, 1992; Ax, 1999; Nielsen, 2001). Consequently, it must be concluded that both Z132 and Z258 have merely contributed noise to the cladistic analysis of Zravy et al. (1998).

\section{Lack of mitosis in somatic or epidermal cells (eutely)}

Despite frequent reference to eutely, or constancy of cell numbers, in the morphological literature such as zoology textbooks, it is actually a very poorly understood character. Although eutely is traditionally discussed in reference to adult animals, a clue to better understanding the evolutionary and biological significance of constancy of cell numbers may reside in better understanding cell fate determination in metazoan embryos and pre-metamorphic larvae.

\section{Character coding}

Z191; E28; K7 (eutelic epidermis a/p): epidermal mitosis a/p

E10: epidermal mitosis by parenchymal kinetosomecontaining cells $\mathrm{a} / \mathrm{p}$

M28; Z10; A13 (absence mitosis in somatic cells): eutely $a / p$

Table 26. Scoring conflicts of epidermal mitosis.

\begin{tabular}{|c|c|c|c|c|}
\hline & $?$ & & Absent & Present \\
\hline Nematoda & & & E28 & Z191 \\
\hline Acoclomorpha & $\mathrm{Z} 191$ & & $\mathrm{E} 28$ & \\
\hline Tardigrada & & .. & E28 & Z191 \\
\hline Kinorhyncha & & & E28 & Z191 \\
\hline Syndermata & & & $\mathrm{K} 7$ & Z191 \\
\hline Priapulida & E28 & & & Z191 \\
\hline
\end{tabular}

Additional scoring conflicts exist for Platyhelminthes which is scored as having no eutely in Meglitsch \& Schram (1991) but polymorphic for the various subtaxa in Zrzavý et al. (1998).
Table 27. Scoring conflicts of eutely.

\begin{tabular}{llll}
\hline & ? & Absent & Present \\
\hline $\begin{array}{l}\text { Gastrotricha } \\
\text { Priapulida }\end{array}$ & Z10 & & M28 \\
$\begin{array}{l}\text { Kinorhyncha } \\
\text { Gnathostomulida }\end{array}$ & Z10 & Z10 & M28 \\
$\begin{array}{l}\text { Nematomorpha } \\
\text { Mesozoa }\end{array}$ & M28 & & M28 \\
\hline
\end{tabular}

Character scoring and phylogenetic significance

Based on the abundance of character scoring conflicts for eutely of epidermis or whole organisms, it is obvious that further detailed comparative study is required to evaluate the phylogenetic significance and evolution of eutely in the Metazoa. Although I made no effort here to resolve the observed scoring conflicts, a number of comments can be made.

Various, typically small-bodied phyla, e.g., the aschelminths, tardigrades, appendicularian urochordates, are thought to be characterized by constancy in cell numbers, either of all somatic cells (eutely) or of selected tissues or organs such as the epidermis. Eutely is related to the lack of mitosis in differentiated cell types, but taxa that lack mitosis in selected body parts are not necessarily eutelic. The platyhelminths provide a clear example. Platyhelminthes lack mitosis in the epidermis or even all differentiated cells (Littlewood et al., 1999a; L49). This is correlated with the presence of neoblasts that function as replacement cells responsible for the maintenance of a healthy population of differentiated somatic cells (Rieger, 1996c; Gschwentner ef al., 2001).

The presence of eutelic tissues, organs or entire organisms is difficult to verify. Large-bodied (macroscopic) metazoans pose an obvious challenge. It is difficult to precisely determine cell numbers in a large organism, and available comparative data suggest that cell number variability is higher in larger species (Azevedo \& Leroi, 2001). Data matrix entries of eutely in large-bodied animals should therefore be interpreted with caution. For example, M28 scored eutely present in priapulids and acanthocephalans. For the priapulids it is probable that at the very least the macrobenthic species are not eutelic (Lorenzen, 1996d), as is suggested by the possession of a body cavity filled with free cells (erythro- 
cytes and amebocytes; Storch, 1991) that are likely to be replenished throughout life, and the documentation of regenerative abilities (Hyman, 1951b: 195; Land, 1975). Although most extant priapulid species are macrobenthic, the plesiomorphic body size of the priapulids cannot be determined with certainty when only extant taxa are considered. The two phyla that are most closely related to the priapulids are the microscopical kinorhynchs and loriciferans. However, a clearer picture emerges when fossil priapulids are also considered. Wills (1998) performed a cladistic analysis of extant and fossil priapulids, also including several Cambrian palaeoscolecidans. His analyses indicated with reasonably certainty that the extant meiobenthic priapulids are derived within the Priapulida.

Similarly, the acanthocephalans may reach considerable body size, ranging from a few to as much as $70 \mathrm{~cm}$ in some species. Interestingly, recent molecular (18S rDNA) and morphological phylogenetic data (Near et al., 1998; Near, 2002; GarcíaVarela et al., 2000, 2002; Monks, 2001; but see the phylogeny of Herlyn et al., 2003) indicate that the largest acanthocephalans are found in the basalmost taxon (which may be paraphyletic), the Archiacanthocephala. This may indicate that small body size and the possibility of eutely may have evolved within this phylum.

It is therefore logical to expect a bias of reliable information for small-bodied taxa, given the difficulties of determining exact cell numbers in largebodied taxa. Cunha et al. (1999) and Azevedo et al. (2000) showed that application of a strict definition of eutely cannot even be applied to the paragon of eutelic metazoans, the Nematoda. On the other hand, for many taxa not enough studies have been directed towards determining the exact cell numbers, and it could well be that various eutelic tissues and organs await discovery in different phyla. For example, Shinn (1997) reports a eutelic epidermis in the chaetognaths, a scoring not yet included in any cladistic study. Also generally noneutelic animals may have eutelic tissues and organs. Drosophila wings and leech nerve ganglia, for example, could be considered eutelic, and cell number variability in organs of reputed eutelic animals such as the rotifers (Shull, 1918) or nematodes may actually be higher than cell number variability of selected organs in non-eutelic organisms (Azevedo et al., 2001). Interestingly, although eutely is habitually mentioned for adult animals, pre-metamorphic larvae of non-eutelic macroscopic animals can also exhibit eutely. For example, echinoderm larvae may possess fixed numbers of cells forming skeletal rods, endoderm, muscles, neurons, etc. Davidson et al. (1998: 3287) write:"...excluding the set-aside cells, the [sea urchin] embryo/larva is essentially eutelic: its cells divide only a set number of times and only a few times after cleavage has ended." These data do not increase confidence in eutely as a robust indicator of phylogenetic affinity. Entirely different genetic regulatory machinery underlies the development of eutelic organs in different animals, such as sea urchin larvae or Drosophila wings (Davidson, 2001). In conclusion, the probability of overall homology of eutelic organs in unrelated animals appears negligible (but see Sørensen, 2003 for a suggestion that eutely of particular organs in the syndermates and Micrognathozoa might be a synapomorphy). The homology of eutely of whole organisms remains very poorly documented, giving little support to eutely as a synapomorphy for Platyhelminthes and Gnathifera (minus Micrognathozoa), or for Plathelminthomorpha in Ahlrichs (1997) and Ax (1995), respectively.

\section{Lophotrochozoan Hox cluster}

Rosa et al. (1999) showed that distinct Hox genes could be characterized by specific amino acid residues or peptides that flank, or are part of the homeodomain of the proteins. Sharing such distinct Hox genes could then indicate phylogenetic relatedness between different phyla (see also Balavoine, 1997, 1998; Adoutte et al., 2000). The Hox genes that were proposed to be characteristic of the lophotrochozoans are: Lox2, Lox4, Lox5, Post1 , and Post-2.

\section{Character coding}

ZI59; ZII1 10: lophotrochozoan complement of Hox genes $\mathrm{a} / \mathrm{p}$ 
Character scoring and phylogenetic significance

Although ZI59 scores the presence of a lophotrochozoan Hox cluster in Rhabditophora, it should be noted that at that time not all the supposedly characteristic lophotrochozoan Hox genes had been documented in the platyhelminths, but continual progress is being made (see Saló et al., 2001 for a recent overview of platyhelminth Hox genes). More importantly, however, Telford $(2000,2001)$ warned that the lack of information on Hox genes in the non-bilaterians creates ambiguity with regards to character polarization. Finally, the very restricted scoring of ZI59 clearly indicates that lack of data for most taxa currently prevents the full phylogenetic significance of this promising character to be revealed. Yet, Hox signatures so far known in platyhelminths are at least consistent with a derived spiralian affinity, rather than a basal placement in the Bilateria.

\section{$\underline{\text { Septate junctions }}$}

Septate junctions are a type of occluding junction that bridge the intercellular space between adjacent cells, and they function in sealing off the intercellular spaces from the surrounding environment. They typically appear in electronmicroscopical pictures as parallel oriented septa.

\section{Character coding}

N61; NI3; S3; P3; Z179: septate junctions a/p H6: septate junctions a/cnidarian type/type of remaining taxa/acoelomorph type

B4: septate/tight junctions $a / p$

BI4: epidermal epithelia with septate or tight junctions $\mathrm{a} / \mathrm{p}$

\section{Character scoring and phylogenetic significance}

Septate junctions have been described for both the calcareans and silicisponges (Green \& Bergquist, 1982; Harrison \& De Vos, 1991), contrary to the scoring of Z179.

Hernandez-Nicaise (1991) report that septate junctions have not been found in the ctenophores, contra N61, NI3, and S3.
Table 28. Scoring conflicts for septate junctions.

\begin{tabular}{llll}
\hline & Absent & Present & $?$ \\
\hline Porifera & $\begin{array}{l}\text { Z179 } \\
\text { (Calcarea) }\end{array}$ & $\begin{array}{l}\text { N61; NI3; } \\
\text { S3 P3 }\end{array}$ & $\begin{array}{l}\text { Z179 } \\
\text { (Silicispon- } \\
\text { gea) }\end{array}$ \\
Ctenophora & Z179; H6; & N61; NI3; & \\
& P3 & S3 & \\
Catenulida & & Z179 & H6 \\
Gnathostomulida & & Z179; S3 & H6 \\
Lobatocerebrum & & Z179 & H6 \\
Entoprocta & & N61; NI3; & H6 \\
Myzostomida & & S3; P3; Z179 & \\
Echiura & & Z179 & H6 \\
& & N61; NI3; & H6 \\
& & S3; P3; Z179 & \\
\hline
\end{tabular}

I have not been able to resolve the scoring conflict between $\mathrm{Z} 179$ and $\mathrm{H} 6$ for the catenulids.

In contrast to H6, a septate junctional complex has been described for the gnathostomulids, albeit weakly developed (Rieger \& Mainitz, 1975).

In contrast to $Z 179$, the presence of septate junctions in Lobatocerebrum remains to be confirmed (Rieger, 1981).

I have not been able to resolve the scoring conflict between $\mathrm{H} 6$ and the other analyses for entoproct septate junctions.

Contra H6, septate junctions have been demonstrated in the myzostomids (Eeckhaut, 1995).

Contra H6, septate junctions have been demonstrated for the echiurans (Schuchert \& Rieger, 1990).

Septate junctions have been described for virtually all phyla, including Porifera and Placozoa. A striking exception is Ctenophora, which appears to lack septate junctions completely, however, the phylogenetic significance of this finding remains uncertain. The interpretation of septate junctions as an autapomorphy for Metazoa therefore remains probable.

\section{Compound cilia}

Compound cilia are composed of closely apposed single cilia that are borne on either monociliate, e.g., phoronid actinotroch larva, or multiciliate cells, e.g., prototroch of trochophore larvae, and they beat as a unit, functioning in feeding and/or locomotion. 
Table 29. Scoring conflicts for compound cilia.

\begin{tabular}{lllll}
\hline & Absent & Present & ? & Proposed scoring \\
\hline Ctenophora & H5 & & Z186; S13 & Present \\
Enteropneusta & Z186; ZI24 (Hemichordata) & S13 & & Present \\
Ectoprocta & Z186 & ZI24 & Absent \\
Gastrotricha & Z186; ZI24 & S13 & $?$ \\
\hline
\end{tabular}

\section{Character coding}

H5; Z186; ZI24: compound cilia a/p

S13: epidermis with compound cilia on multiciliate cells a/p

\section{Character scoring and phylogenetic significance}

The scorings of the characters on compound cilia in recent cladistic analyses are primarily based upon the detailed studies of Nielsen $(1987,1995,2001)$, and it thus appears that the observed scoring conflicts reflect divergent interpretations of his data.

Ctenophoran comb plates are composed of large compound cilia arising from several multiciliate cells, but their unique structure and function make evaluation of their homology with other compound cilia difficult, so that all observed scorings appear to be justified. Curiously though, none of the studies actually scored ctenophoran compound cilia as present to test their homology to other ciliary systems of compound cilia by character congruence.

The telotroch (called archaeotroch by Nielsen) of enteropneust planktotrophic tornaria larvae is composed of compound cilia on multiciliate cells. Because a telotroch is also a conspicuous feature of the lecithotrophically, "directly" developing harimaniids (Hadfield, 1975; Goldschmid, 1996b) it can be assumed that a telotroch is an enteropneust ground pattern character, even though the phylogeny of the group remains unclear (but see Halanych, 1995; Cameron et al., 2000; Peterson ét al., 2000a; Peterson \& Eernisse, 2001).

Although the ectoprocts have multiciliate epidermal cells, they do not form compound cilia (Nielsen, 1987, 1998b; Nielsen \& Riisgård, 1998), contra ZI24.

Compound cilia have been reported in the gastrotrichs (Nielsen, 1987 based on Rieger, 1976), but their presence in the gastrotrich ground pattern appears uncertain. Note that basal gastrotrich taxa all possess monociliate epidermal cells (Hochberg \& Litvaitis, 2000).

Sørensen et al. (2000) scored Micrognathozoa as '?' for compound cilia. This scoring can be changed, since it has been found that the so-called head and trunk ciliophores, of which at least the latter are used for locomotion, consist of compound cilia (Kristensen \& Funch, 2000).

One can find various other scoring conflicts for compound cilia'in the recent literature, for example for the urochordates, echinoderms, pterobranchs, ecdysozoans, acanthocephalans, gnathostomulids, clitellates, and platyhelminths, but with the exception of the last phylum where multiciliate cells are present but compound cilia have not been reported, all these apparent conflicts merely reflect whether taxa without epidermal cilia or with only monociliate cells, should be scored 'inapplicable' or 'absent' for compound cilia. Although different decisions may effect different phylogenetic results, these alternative scorings can all be defended. However, if one accepts the possible homology of compound cilia arising from both monociliate cells (reported for phoronid actinotroch larvae and an anthozoan Zoanthina larva; Nielsen, 1987) and multiciliate cells (a proposal not followed in any of the studies considered here), than it would be inappropriate to score taxa with monociliate cells as 'inapplicable.'

The morphological analysis of Zrzavý et al. (2001) suggest a single evolutionary origin of compound cilia, which contrasts with the two independent origins suggested by Zrzavý et al. (1998) (for rotifers and the rest) and Sørensen et al. (2000) (for enteropneusts and the rest). The evolution of compound cilia within Anthozoa, probably Gastrotricha, and Nemertea (only observed in pilidium larvae that are not likely plesiomorphic for the phylum), indicate that the homology of compound cilia across the Metazoa is unlikely. Further analyses at least 
incorporating the proper scorings for both the enteropneusts and Micrognathozoa may further help us to decide whether compound cilia may be at least homologous within the protostomes.

\section{Respiratory pigments}

Respiratory pigments is a catch-all term for oxygen transporting proteins that may either occur freely (extracellular) dissolved in the body fluids (hemoglobin, hemocyanin) or in 'blood' cells (hemoglobin, hemerythrin).

\section{Character coding}

$\mathrm{H} 24$; Z44: respiratory pigments (called blood pigments in Haszprunar, 1996a) a/p

Ca49: no special respiratory pigments/hemoglobin/ hemerythrin

P115; ZII113: hemerythrin a/p

Table 30. Scoring conflicts of respiratory pigments.

\begin{tabular}{lll}
\hline & Absent & Present \\
\hline Pterobranchia & $\mathrm{Ca} 49$ & Z44 \\
Myzostomida & $\mathrm{H} 24$ & Z44 \\
\hline
\end{tabular}

\section{Character scoring and phylogenetic significance}

Although free blood pigments reminiscent of hemoglobin have been reported from some species of the enteropneusts (Benito \& Pardos, 1997), they have apparently not been reported for the pterobranchs, contra Z44.

Being unable to find any reports supporting the scoring of respiratory pigments in the myzostomids, I tentatively support the scoring of $\mathrm{H} 24$.

Jenner (2001a) discussed some additional scoring problems of $\mathrm{Z} 44$, but a more important problem is that of inappropriate character coding for $\mathrm{Z} 44, \mathrm{H} 24$, and $\mathrm{Ca} 49$. These characters code arguably non-homologous respiratory proteins as parts of a single character, at least hemoglobins and hemerythrin. Molecular support for this assumption is lacking. Current molecular phylogenetic data instead suggests that the different oxygen-transporting proteins have evolved independently from each other, including separate origins of the arthropod and molluscan hemocyanins, although these latter two may share a more distant common ancestry (Holde, 1997; Holde et al., 2001; Burmester, 2001; Kusche et al., 2002).

\section{Gliointerstitial cell system}

This is a system of granular cells that connects the system of glia cells in the central nervous system with glia-like cells elsewhere in the body, often where the peripheral nervous system connects to other tissue such as epidermis (then called basal cells), gastrodermis, or muscles, but glia-like cells sometimes also occur as free cells.

\section{Character coding}

H16; HaXIIa; P110; Z223; ZI50, ZII98: gliointerstitial cell system $\mathrm{a} / \mathrm{p}$

\section{Character scoring and phylogenetic significance}

This character was introduced into metazoan cladistics by Haszprunar (1996a, b) and his scoring has been adopted in various later studies (Zrzavý et al., 1998, 2001; Peterson \& Eernisse, 2001). The gliointerstitial system was first and best described in the molluscs and annelids (see review in Rieger, 1981), but potentially homologous cells are scored as present in Lobatocerebrum, nemerteans, echiurans, and sipunculans. Turbeville (2002) reports that all major nemertean taxa have a gliointerstitial cell system, and that this system is absent in the nonbilaterians, platyhelminths, and the deuterostomes. The scoring of gliointerstitial cells in deuterostomes in Peterson \& Eernisse (2001) was changed to absent in an unpublished new version of their matrix (K. J. Peterson, pers. comm.). The currently adopted character scoring probably does not properly reflect the phylogenetic significance of this feature. First, glia cells (which form part of the gliointerstitial system) associated with the central nervous system have a much wider distribution among the Metazoa, as they occur in all major protostomian and deuterostomian clades, e.g., the echinoderms, chaetognaths, gastrotrichs, platyhelminths (Rieger et al., 1991b; Ruppert, 1991b; Byrne, 1994; Shinn, 1997), and they may come in an impressive vari- 
ety of forms, as for example in the arthropods (Carlson, 1987). Second, equally compelling evidence for the presence of a gliointerstitial system as exists for echiurans and sipunculans, is available for several other phyla, including the brachiopods, cycliophorans, cephalochordates, and especially vestimentiferans (contra the scoring of Z223, P110) (Gardiner \& Jones, 1993; Funch \& Kristensen, 1997; James, 1997; Ruppert, 1997). In these taxa, glia cells are observed at neuro-muscular synapses (Cycliophora), in association with nerves in the connective tissue (Brachiopoda, Cephalochordata), and as part of a well-developed basal cell system (Vestimentifera). I therefore conclude that further study of this character is necessary for a complete elucidation of its evolutionary history.

\section{Conclusions: progress and prospects}

Contemporary morphological cladistic analyses of the Metazoa are seriously flawed. The process of distilling phylogenetic signal from a given matrix typically receives disproportionate attention when compared with the efforts invested in the compilation of a reliable data set. In this paper I show that recent morphological phylogenetic analyses of metazoan relationships are riddled with character scoring errors. Similarly, character coding has so far received only minimal attention (Jenner, 2002). Metazoan cladistics has to be properly rebalanced by returning comparative morphology to the center of attention. The health of phylogenetic hypotheses ultimately depends on the vigor of their empirical lifeline. Although this is generally endorsed in theory, in actuality current practice can be significantly improved.

This study attempts a first step to redress the balance of metazoan cladistics. The character support for all competing sister group relationships of the 'acoelomate' worms Platyhelminthes, Nemertea, and Gnathostomulida is evaluated. All conflicts in the scoring of characters shared between cladistic analyses are identified, and where possible resolved. The overall phylogenetic significance of the characters is discussed, and gaps in our understanding of the characters and knowledge of their distribu- tions across the Metazoa are pointed out. Several conclusions can be drawn.

Progress in our understanding of the phylogenetic placement of the 'acoelomate' worms is based on the incorporation of new empirical data into cladistic data matrices. For example, the incorporation of characters on the ultrastructural similarities of gnathiferan jaw elements into the most recent studies, implies that older studies (Meglitsch \& Schram, 1991; Eernisse et al., 1992), for which much of this information was not available, can no longer be considered as effective summaries of current evidence. This study also shows that our knowledge of many characters for many phyla should be improved significantly before we can hope to fill our matrices with unambiguous character scorings.

It is much more difficult to identify true progress in our understanding of the overall placements of the 'acoelomate" worms within the Metazoa because the published analyses" differ substantially with regard to various analysis parameters, notably character choice and character scoring. Many characters introduced into metazoan cladistics at a certain time are only incorporated into some of the more recent studies, or they may be excluded from all newer studies altogether. However, character selection criteria are typically not made explicit. Consequently, an effectively random pattern of character selection becomes obvious when different studies are compared. This can be observed for characters coding for mode of sperm deposition, mode of fertilization, sperm morphology, gonad organization, blastula, larval ciliary bands, coelom, coelomogenesis, cerebral ganglia, statocysts, protonephridia, muscle cytology, compound cilia, and so forth. A comparison of character selection in two successive studies by the same research group provides a convincing epitome. Of the 18 synapomorphies supporting sister group relationships of platyhelminths, nemerteans, and gnathostomulids in Zrzavý et al. (1998), only two were also coded in Zrzavý et al. (2001)! Two additional characters in the latter study are reminiscent but not identical to characters coded in the first study. Strikingly, Zrzavý et al. (2001) provide no explanation at all for the exclusion of more than $75 \%$ of the characters that proved to be most relevant for placing 'acoelomate' worms in their previous analysis. 
Obviously, given such lack of transparency and uncritical compilation of the data matrix, it seems hardly possible to claim an increase in our understanding of metazoan relationships, rather than a mere change of opinions.

During personal communications with several colleagues, the surprising opinion has repeatedly been aired that as long as the percentage of scoring errors in a given matrix is not too high, the cladistic analysis may not be fatally weakened. Unfortunately, this suggestion rests on a misunderstanding of the nature of cladistic data matrices. The overall phylogenetic accuracy of a cladistic analysis is not simply inversely correlated with the percentage of errors because a matrix does not contain a single phylogenetic signal. A data matrix is a mosaic of characters with distinct phylogenetic signals at different levels. This hierarchical structure in the matrix implies that the proper placement of different taxa to an important extent depends on different characters. Any errors in the set of features most important for placing a certain phylum (or supraphyletic clade), even when they constitute only a minute percentage of the overall information content of the matrix, will mislead us about the relationships of these taxa.

It has been shown that single decisions about character coding can have profound effects on the outcome of the analyses. This became apparent, for example, for the decision to code sperm acrosome and subacrosomal material (perforatorium) as either one or two characters in the matrix of Peterson \& Eernisse (2001). Similarly, incorrect character scorings can distort the outcomes of a cladistic analysis. As discussed above, rescoring of one taxon for muscle type, and two taxa for shape of pharynx lumen in the data matrix of Wallace et al. (1996) resulted in the complete collapse of the strict consensus. Relatively few changes to the contents of a data matrix can result in profound changes in the outcome of a cladistic study (see also Jenner \& Schram, 1999; Jenner, 2001a, 2002; Turbeville, 2002). Consequently, it is impossible to accurately predict the outcome of the analysis before all problematic data entries have been corrected. Because all analyses evaluated here can be adjusted in various ways, it thus becomes impossible to say at this point whether the heretofore published results will withstand scrutiny.

We can now see that further study is necessary to elucidate the overall phylogenetic significance of many characters. Several characters have been introduced in cladistic analyses with a restricted sampling of phyla, and despite the fact that later more comprehensive studies took over these characters, this has not always been accompanied by a careful reconsideration of character scoring, e.g., the characters concerning the presence of cerebral ganglia, cuticle layers, and coelomocytes. Other characters blend a proper morphological component with an unwarranted assumption about evolutionary transformation, e.g. a larva with reduced hyposphere for the parenchymians, reduction of the hindgut in the platyhelminths, and presence of an orthogonal nervous system in the nematodes. Other characters appear to uphold an artificial dichotomy between broadly protostomian and deuterostomian organization, such as the source of mesoderm and possession of a dorsal brain. Still other characters only introduce noise into the analyses because there appears to be no reason for primary homology, e.g. prototrochal lobes in the larvae of nemerteans and molluscs, whereas overall homology of other characters across the Metazoa is very doubtful, viz., filiform sperm, and internal fertilization. The scoring of all these characters merits special attention in future cladistic analyses.

Given that phylogenetic analyses of $18 \mathrm{~S}$ rDNA sequences have proven not to be the panacea for placing the 'acoelomate' worms, continued attention to morphological evidence remains crucial. A future consensus is dependent upon the continued reassessment of morphological data included in cladistic data matrices. Uncertainties about the ground pattern character states for the phyla (due to both the paucity of morphological information within taxa, and uncertainty about their internal phylogenetic relationships), differing assumptions about semaphoront choice, and different decisions about character coding make this a very challenging task.

The observation that the different cladistic analyses exhibit substantial differences in character selection leads to the conclusion that the unique strength of cladistics to arbitrate between alternative hypotheses has not yet been fully exploited. 
To achieve this, all pertinent information has to be included into a single analysis to prevent the results from being determined by biases in the selection of input data (see also Jenner, in press). If our goal is to translate time's arrow of merely changing opinions about metazoan phylogeny into genuine progress in understanding, we have to abandon the use of cladistics as an easy tool to generate "novel" hypotheses of metazoan relationships, and employ cladistics more critically as an effective instrument to test the relative merit of available multiple alternative hypotheses.

\section{Acknowledgements}

The bulk of this project was done at the Institute for Biodiversity and Ecosystem Dynamics at the University of Amsterdam with the help of grant 805-33.431-P from the Earth and Life Sciences Foundation (ALW) of the Netherlands Organization for Scientific Research (NWO). My work at the University of Cambridge is supported by a Marie Curie Individual Fellowship of the European Community program Improving Human Potential under contract number HPMF-CT-2002-01712. This big endeavor was greatly facilitated by the help of several colleagues. I want to thank Dr. Martin Sørensen for his valuable comments on the manuscript, and information on cycliophoran sperm. I wish to thank Dr. Rudolf Nieuwenhuys for many invigorating discussions. I wish to thank Dr. Kevin Peterson for providing an unpublished modified version of the data matrix of Peterson \& Eernisse (2001), and Drs. Jan Zrzavý, Claus Nielsen, and Rick Hochberg for providing digital versions of the data matrices of Zrzavý et al. (1998), Zrzavý (2003), Nielsen (2001), and Hochberg \& Litvaitis (2000). Drs. Jo van den Biggelaar and Peter Damen graciously shared unpublished information on the embryology of Convoluta sp. and Patella, respectively. I thank Dr. Anke Klerkx for discussing her promotor construct experiments in various spiralians. Dr. Reinhardt Kristensen provided information about the reproductive mode of Loricifera, and Dr. Claus Nielsen shared information about the entoprocts. I thank Dr. Andreas SchmidtRhaesa for sharing his knowledge of nematomorphs, and Dr. Jon Norenburg for sharing unpublished information about the phylogeny of the nemerteans. Finally, special thanks to Dr. Frederick Schram for his unfailing support throughout this project, and for his exhaustive reading of various bulky drafts of this paper. Fred, I promise never to do this again... This work is publication No. 12 of the Dutch national program in systematics and developmental biology.

\section{References}

Adiyodi KG, Adiyodi RG. 1983. Reproductive biology of invertebrates. Chichester: John Wiley \& Sons.

Adoutte A, Balavoine G, Lartillot N, Rosa R. de. 1999. Animal evolution, the end of intermediate taxa? TIG 15: 104108.

Adoutte A, Balavoine $G$, Lartillot $N$, Lespinet $O$, Prud'homme B, Rosa R. de. 2000. The new animal phylogeny: reliability and implications. Proc. Natl. Acad. Sci. US.A 97: 4453-4456.

Adrianov AV, Malakhov VV. 1996. Priapulida (Priapulida): structure, development, phylogeny, and classification. KMK Scientific Press.

Aguinaldo AMA, Turbeville JM, Linford LS, Rivera MC, Garey JR, Raff RA, Lake JA. 1997. Evidence for a clade of nematodes, arthropods and other moulting animals. Nature 387: 489-493.

Aguinaldo AMA, Lake JA. 1998. Evolution of the multicellular animals. American Zoologist 38: 878-887.

Ahlrichs WH. 1993a. On the protonephridial system of the brackish-water totifer Proales reinhardti (Rotifera, Monogononta). Microfauna Marina 8: 39-53.

Ahlrichs WH. 1993b. Ultrastructure of the protonephridia of Seison annulatus (Rotifera). Zoomorphology 113: 245-251.

Ahlrichs WH. 1995. Ultrastruktur und Phylogenie von Seison nebaliae (Grube 1859) und Seison annulatus (Claus 1876). Hypothesen zu phylogenetischen Verwandtschaftsverhältnissen innerhalb der Bilateria. Göttingen: Cuvillier Verlag.

Ahlrichs WH. 1997. Epidermal ultrastructure of Seison nebaliae and Seison annlulatus, and a comparison of epidermal structures within the Gnathifera. Zoomorphology 117: 41-48.

Almeida WO, Christoffersen ML. 2000. Análise cladística dos grupos basais de Metameria: uma nova proposta para o posicionamento dos Arthropoda e grupos afins entre os poliquetos errantes. Holos: Ribeirão Preto.

Alvariño A. 1983. Chaetognatha. In: Adiyodi KG, Adiyodi RG, eds. Reproductive biology of invertebrates . Chichester: John Wiley \& Sons, 531-544.

Alvariño A. 1990. Chaetognatha. In: Adiyodi KG, Adiyodi RG, eds. Reproductive biology of invertebrates Vol IV, Part B . Chicester: John Wiley \& Sons, 255-282.

Alvestad-Graebner I, Adam H. 1983. Gnathostomulida. In: Adiyodi KG, Adiyodi RG, eds. Reproductive biology of invertebrates . Chichester: John Wiley \& Sons, 171-180.

Anderson DT. 1969. On the embryology of the cirripede crustaceans Tetraclita rosea (Krauss), Tetraclita purpurascens (Wood), Chthamalus antennatus (Darwin) and Chamaesipho columna (Spengler) and some considerations of crustacean phylogenetic relationships. Phil. Trans. Roy. Soc. Lond. B 256: $183-235$.

Anderson DT. 1973. Embryology and phylogeny in annelids and arthropods. Oxford: Pergamon Press.

Anderson DT. 1981. Origins and relationships among the animal phyla. Proc. Linn. Soc. New South Wales 106: 151-166.

Anderson DT. 1998. The Sipuncula and Priapula. In: Anderson DT, ed. Invertebrate Zoology. Oxford: Oxford University Press, 116-121. 
Arendt D, Nübler-Jung K. 1994. Inversion of dorsoventral axis? Nature 371: 26.

Arendt D, Nübler-Jung K. 1996. Common ground plans in early brain development in mice and flies. BioEssays 18: 255-259.

Arendt D, Nübler-Jung K. 1997. Dorsal or ventral: similarities in fate maps and gastrulation patterns in annelids, arthropods and chordates. Mech. Dev. 61: 7-21.

Arendt D, Nübler-Jung K. 1999a. Comparison of early nerve cord development in insects and vertebrates. Development 126: 2309-2325.

Arendt D, Nübler-Jung K. 1999b. Rearranging gastrulation in the name of yolk: evolution of gastrulation in yolk-rich amniote eggs. Mech. Dev. 81: 3-22.

Arendt D, Wittbrodt J. 2001. Reconstructing the eyes of Urbilateria. Phil. Trans. Roy. Soc. Lond. B 356: 1545-1563.

Arendt D, Tessmar K, Medeiros de Campos-Baptista MI, Dorresteijn A, Wittbrodt J. 2002. Development of pigmentcup eyes in the polychaete Platynereis dumerilii and evolutionary conservation of larval eyes in Bilateria. Development 129: 1143-1154.

Arendt D, Technau U, Wittbrodt J. 2001. Evolution of the bilaterian larval foregut. Nature 409: 81-85.

Arnold EN. 1990. Why do morphological phylogenies vary in quality? An investigation based on the comparative history of lizard clades. Proc. R. Soc. Lond. B 240: 135-172.

Ax P. 1985. The position of the Gnathostomulida and Platyhelminthes in the phylogenetic system of the Bilateria. In: Conway Morris S, George JD, Gibson R, Platt HM, eds.The origins and relationships of lower invertebrates. Oxford: Clarendon Press, 168-180.

Ax P. 1989. Basic phylogenetic systematization of the Metazoa. In: Fernholm B, Bremer K, Jörnvall H, eds. The Hierarchy of life. Molecules and Morphology in phylogenetic analysis. Proceedings from Nobel Symposium 70, 1988. Amsterdam: Excerpta Medica/Elsevier, 229-245.

Ax P. 1995. Das System der Metazoa I. Ein Lehrbuch der phylogenetischen Systematik. Stuttgart: Gustav Fischer Verlag.

Ax P. 1999. Das System der Metazoa II. Ein Lehrbuch der phylogenetischen Systematik. Stuttgart: Gustav Fischer Verlag.

Azevedo RBR, Cunha A, Emmons SW, Leroi AM. 2000. The demise of the platonic worm. Nematology 2: 71-79.

Azevedo RBR, Leroi AM. 2001. A power law for cells. Proc. Natl. Acad. Sci. USA 98: 5699-5704.

Backeljau T, Winnepenninckx B, De Bruyn L. 1993. Cladistic analysis of metazoan relationships: a reappraisal. Cladistics 9: 167-181.

Baguñà J, Ruiz-Trillo I, Paps J, Loukota M, Ribera C, Jondelius U, Riutort M. 2001. The first bilaterian organisms: simple or complex? New molecular evidence. Int. J. Devel. Biol. 45: S133-S134.

Bakke T. 1990. Pogonophora. In: Adiyodi KG, Adiyodi RG, eds. Reproductive biology of invertebrates. Vol IV, Part B. Chicester: John Wiley \& Sons, 37-48:

Balavoine G. 1997. The early emergence of platyhelminths is contradicted by the agreement between $18 \mathrm{~S}$ rRNA and Hox genes data. C. R. Acad. Sci. Paris 320: 83-94.

Balavoine G. 1998. Are Platyhelminthes coelomates without a coelom? An argument based on the evolution of Hox genes. Amer. Zool. 38: 843-858.
Balavoine G, Adoutte A. 1998. One or three Cambrian radiations? Science 280: 397-398.

Balfour FM. 1880. Larval forms: their nature, origin, and affinities. Quart. J. Micros. Sci. 20: 381-407.

Bartolomaeus T. 1985. Ultrastructure and development of the protonephridia of Lineus viridis (Nemertini). Microfauna Marina 2: 61-83.

Bartolomaeus T. 1989a. Ultrastructure and relationship between protonephridia and metanephridia in Phoronis muelleri (Phoronida). Zoomorphology 109: 113-122.

Bartolomaeus T. 1989b. Larvale Nierenorgane bei Lepidochiton cinereus (Polyplacophora) und Aeolidia papillosa (Gastropoda). Zoomorphology 108: 297-307.

Bartolomaeus T. 1989c. Ultrastructure and development of the nephridia in Anaitides mucosa (Annelida, Polychaeta). Zoomorphology 109: 15-32.

Bartolomaeus T. 1993a. Die Leibeshöhlenverhältnisse und Verwandtschaftsbeziehungen der Spiralia. Verh. Deutsch. Zool. Ges. 86: 42.

Bartolomaeus T. 1993b. Ultrastructure of the protonephridia in the larva of Autolytus prolifer (Annelida, Syllidae): implications for annelid phylogeny. Microfauna Marina 8: 55-64.

Bartolomaeus T. 1994. On the ultrastructure of the coelomic lining in the Annelida, Sipuncula and Echiura. Microfauna Marina 9: 171-220.

Bartolomaeus T. 1995. Ultrastructure of the protonephridia in larval Magelona mirabilis (Spionida) and Pectinaria auricoma (Terebellida): head kidneys in the ground pattern of the Annelida. Microfauna Marina 10: 117-141.

Bartolomaeus T. 1996/1997. Ultrastructure of the renopericardial complex of the interstitial gastropod Philinoglossa helgolandica Hertling, 1932 (Mollusca: Opisthobranchia). Zool. Anz. 235: 165-176.

Bartolomaeus T. 1998. Head kidneys in hatchlings of Scoloplos armiger (Annelida: Orbiniida): implications for the occurrence of protonephridia in lecithotrophic larvae. J. Mar. Biol. Ass. United Kingdom 78: 183-192.

Bartolomaeus T. 1999. Structure, function and development of segmental organs in Annelida. Hydrobiologia 402: 21-37.

Bartolomaeus T. 2001. Ultrastructure and formation of the body cavity lining in Phoronis muelleri (Phoronida, Lophophorata). Zoomorphology 120: 135-148.

Bartolomaeus T, Ax P. 1992. Protonephridia and metanephridia - their relation within Bilateria. Z. zool. Syst. Evolutionsforsch. 30: 21-45.

Bartolomaeus T, Ruhberg H. 1999. Ultrastructure of the body cavity lining in embryos of Epiperipatus biolleyi (Onychophora, Peripatidae) - a comparison with annelid larvae. Invert. Biol. 118: 165-174.

Beer A-J, Moss C, Thorndyke M. 2001. Development of serotonin-like and SALMFamide-like immunoreactivity in the nervous system of the sea urchin Psammechinus miliaris. Biol. Bull. 200: 268-280.

Bely AE, Wray GA. 1999. Expression of homeobox genes during regeneration and asexual reproduction in an annelid. Amer. Zool. 39: 12A.

Benito J, Pardos F. 1997. Hemichordata. In: Harrison FW, Ruppert EE, eds. Microscopic anatomy of invertebrates. Vol. 
15. Hemichordata, Chaetognatha, and the invertebrate chordates. New York: Wiley-Liss, 15-101.

Bergström J. 1989. The origin of animal phyla and the new phylum Procoelomata. Lethaia 22: 259-269.

Bergström J. 1997. Origin of high-rank groups of organisms. Paleont. Res. 1: 1-14.

Bergström J, Naumann WW, Viehweg J, Martí-Mus M. 1998. Conodonts, calcichordates and the origin of vertebrates. Mitt. Mus. Nat.kd. Berl. Geowiss. 1: 81-92.

Berney C, Pawlowski J, Zaninetti L. 2000. Elongation factor 1-alpha sequences do not support an early divergence of the Acoela. Mol. Biol. Evol. 17: 1032-1039.

Bertolani R. 1990. Tardigrada. In: Adiyodi KG, Adiyodi RG, eds. Reproductive biology of invertebrates. Vol IV, Part B. Chicester: John Wiley \& Sons, 49-60.

Biggelaar JAM van den, Loon AE van, Damen WGM. 1996. Mesentoblast and trochoblast specification in species with spiral cleavage predict their phyletic relations. Nether. J. Zool. 46: 8-21.

Biggelaar JAM van den, Dictus WJAG, Loon AE van. 1997. Cleavage patterns, cell-lineages and cell specification are clues to phyletic lineages in Spiralia. Semin. Cell \& Devel. Biol. 8: 367-378.

Biggelaar JAM van den, Edsinger-Gonzales E, Schram Fr. 2002. The improbability of dorso-ventral axis inversion during animal evolution. Contr. Zool. 71: 29-36.

Biggelaar JAM van den, Haszprunar G. 1996. Cleavage patterns and mesentoblast formation in the Gastropoda: an evolutionary perspective. Evolution 50: 1520-1540.

Bird AF, Sommerville RI. 1989. Nematoda and Nematomorpha. In: Adiyodi KG, Adiyodi RG, eds. Reproductive biology of invertebrates. Vol IV, Part A. Chicester: John Wiley \& Sons, 219-250.

Boero F, Gravili C, Pagliara P, Piraino S, Bouillon J, Schmid V. 1998. The cnidarian premises of metazoan evolution: from triploblasty, to coelom formation, to metamery. Ital. J. Zool. 65: 5-9.

Bogitsh BJ, Harrison FW. 1991. Introduction. In: Harrison FW, Bogitsh BJ, eds. Microscopic anatomy of invertebrates. Vol. 3. Platyhelminthes and Nemertinea. New York: Wiley-Liss, $1-5$.

Boury-Esnault N, Efremova S, Bezac C, Vacelet J. 1999. Reproduction of a hexactinellid sponge: first description of gastrulation by cellular determination in the Porifera. Invert. Reprod. \& Devel. 35: 187-201.

Bovee EC. 1991. Sarcodina. In: Harrison FW, Corliss JO, eds. Microscopic anatomy of invertebrates. Vol. 1. Protozoa. New York: Wiley-Liss, 161-259.

Boyer BC. 1997. 2. Mechanisms of development in the polyclad Turbellaria: a key to understanding the evolution of development in the Spiralia. In: Adiyodi KG, Adiyodi RG, eds. Reproductive biology of invertebrates. Vol VII. Oxford: Oxford \& IBH Publishing Co. Pvt. Ltd, 43-65.

Boyer BC, Henry JQ. 1998. Evolutionary modifications of the spiralian developmental program. Amer. Zool. 38: 621-633.

3oyer BC, Henry JQ, Martindale MQ. 1996a. Dual origins of mesoderm in a basal spiralian: cell lineage analyses in the polyclad turbellarian Hoploplana inquilina. Dev. Biol. 179: 329-338.
Boyer BC, Henry JQ, Martindale MQ. 1996b. Modified spiral cleavage: the duet cleavage pattern and early blastomere fates in the acoel turbellarian Neochildia fusca. Biol. Bull. 191: 285-286.

Boyer BC, Henry JJ, Martindale MQ. 1998. The cell lineage of a polyclad turbellarian embryo reveals close similarity to coelomate spiralians. Dev. Biol. 204: 111-123.

Brahmachary RL. 1989. Mollusca. In: Adiyodi KG, Adiyodi RG, eds. Reproductive biology of invertebrates. Vol IV, Part $A$. Chicester: John Wiley \& Sons, 281-348.

Bresciani J. 1991. Nematomorpha. In: Harrison FW, Ruppert EE, eds. Microscopic anatomy of invertebrates. Vol. 4. Aschelminthes. New York: Wiley-Liss, 197-218.

Bridge D, Cunningham CW, DeSalle R, Buss LW. 1995. Classlevel relationships in the phylum Cnidaria: molecular and morphological evidence. Mol. Biol. Evol. 12: 679-689.

Brusca RC, Brusca GJ. 1990. Invertebrates. Massachusetts Sinauer Associates.

Brusca RC, Brusca GJ. 2003. Invertebrates. Second edition Massachusetts: Sinauer Associates.

Brusca GJ, Brusca RC, Gilbert SF. 1997. Characteristics of metazoan development. In: Gilbert, SF, Raunio AM, eds. Embryology. Constructing the organism. Sunderland: Sinauer Associates, 3-19.

Buckland-Nicks J, Scheltema A. 1995. Was internal fertilization an innovation of early Bilateria? Evidence from sperm structure of a mollusc. Proc. R. Soc. Lond. B. 261: 11-18.

Budd GE. 2001. Why are arthropods segmented? Evol. Devel. 3: $332-342$

Budd GE, Jensen S. 2000. A critical reappraisal of the fossil record of the bilaterian phyla. Biol. Rev. 75: 253-295.

Budelmann BU. 1995. The cephalopod nervous system: what evolution has made of the molluscan design. In: Breidbach $\mathrm{O}$, Kutsch W, eds. The nervous system of invertebrates: an evolutionary and comparative approach. Basel: Birkhäuser Verlag, 115-138.

Budelmann BU, Schipp R, Boletzky S. von. 1997. Cephalopoda. In: Harrison FW, Kohn AJ, eds. Microscopic anatomy of invertebrates. Vol. 6A. Mollusca II. New York: Wiley-Liss, 119-414.

Bullock TH. 1965a. Nemertinea. In: Bullock TH, Horridge GA, eds. Structure and function in the nervous systems of invertebrates. San Francisco: W.H. Freeman and Company, 576595.

Bullock TH. 1965b. Coelenterata and Ctenophora. Bullock TH, Horridge GA, eds. Structure and function in the nervous systems of invertebrates. San Francisco: W.H. Freeman and Company, 459-534.

Bullock TH. 1965c. Pseudocoelomate phyla. Bullock TH, Horridge GA, eds. Structure and function in the nervous systems of invertebrates. San Francisco: W.H. Freeman and Company, 597-629.

Bullock TH. 1965d. Chaetognatha, Pogonophora, Hemichördata, and Tunicata. Bullock TH, Horridge GA, eds. Structure and function in the nervous systents of invertebrates. San Francisco: W.H. Freeman and Company, 1559-1592.

Burighel P, Cloney RA. 1997. Urochordata: Ascidiacea. In: Harrison FW, Ruppert EE, eds. Microscopic anatomy of in- 
vertebrates. Vol. 15. Hemichordata, Chaetognatha, and the invertebrate chordates. New York: Wiley-Liss, 221-347.

Burmester T. 2001. Molecular evolution of the arthropod hemocyanin superfamily. Mol. Biol. Evol. 18: 184-195.

Buss LW. 1983. Evolution, development, and the units of selection. Proc. Natl. Acad. Sci. USA 80: 1387-1391.

Buss LW. 1987. The evolution of individuality. Princeton Princeton University Press.

Byrne M. 1994. Ophiuroidea. In: Harrison FW, Chia F-S, eds. Microscopic anatomy of invertebrates. Vol. 14. Echinodermata. New York: Wiley-Liss, 247-343.

Callsen-Cencic P, Flügel HJ. 1995. Larval development and the formation of the gut of Siboglinum poseidoni Flügel \& Langhof (Pogonophora, Perviata). Evidence of protostomian affinity. Sarsia 80: 73-89.

Cameron CB, Garey JR, Swalla BJ. 2000. Evolution of the chordate body plan: new insights from phylogenetic analyses of deuterostome phyla. Proc. Natl. Acad. Sci. USA 97: 4469-4474.

Campos A, Cummings MP, Reyes JL, Laclette JP. 1998. Phylogenetic relationships of Platyhelminthes based on $18 \mathrm{~S}$ ribosomal gene sequences. Mol. Phyl. Evol. 10: 1-10.

Cantell C-E. 1989. Nemertina. In: Adiyodi KG, Adiyodi RG, eds. Reproductive biology of invertebrates. Vol IV, Part A. Chicester: John Wiley \& Sons, 147-165.

Cantino PD, Bryant HN, De Queiroz K, Donoghue MJ, Eriksson T, Hillis DM, Lee MSY. 1999. Species names in phylogenetic nomenclature. Syst. Biol. 48: 790-807.

Carlson SD. 1987. Ultrastructure of the arthropod neuroglia and neuropil. In: Gupta AP, ed. Arthropod brain. Its Evolution, development, structure, and functions. New York: John Wiley \& Sons, Inc., 323-346.

Carlson SJ. 1995. Phylogenetic relationships among extant brachiopods. Cladistics 11: 131-197.

Carranza S, Baguñà J, Riutort M. 1997. Are the Platyhelminthes a monophyletic primitive group? An assessment using 18S rDNA sequences. Mol. Biol. Evol. 14: 485-497.

Carroll SB, Grenier JK, Weatherbee SD. 2001. From DNA to diversity. Malden: Blackwell Science, Inc.

Cavalier-Smith T. 1998. A revised six-kingdom system of life. Biol. Rev. 73: 203-266.

Cavey MJ, Märkel K. 1994. Echinoidea. In: Harrison FW, Chia F-S, eds. Microscopic anatomy of invertebrates. Vol. 14. Echinodermata. New York: Wiley-Liss, 345-400.

Chia F-S, Bickell LR. 1978. Mechanisms of larval attachment and the induction of settlement and metamorphosis in coelenterates: a review. In: Chia F-S, Rice ME, eds. Settlement and metamorphosis of marine invertebrate larvae. New York: Elsevier, 1-12.

Chia F-S, Bickell LR. 1983. Echinodermata. In: Adiyodi KG, Adiyodi RG, eds. Reproductive biology of invertebrates. Volume II. Chicester: John Wiley \& Sons, 545-620.

Chia FS, Walker CW. 1991. Echinodermata: Asteroidea. In: Giese AC, Pearse, JS, Pearse VB, eds. Reproduction of marine invertebrates. Vol. VI. Echinoderms and lophophorates. California: The Boxwood Press, 301-353.

Chia FS, Koss R. 1994. Asteroidea. In: Harrison FW, Chia F-S, eds. Microscopic anatomy of invertebrates. Vol. 14. Echinodermata. New York: Wiley-Liss, 169-245.
Christoffersen ML, Araújo-de-Almeida E. 1994. A phylogenetic framework of the Enterocoela (Metameria: Coelomata). Rev. Nord. Biol. 9: 173-208.

Chuang SH. 1990. Brachiopoda. In: Adiyodi KG, Adiyodi RG, eds. Reproductive biology of invertebrates. Vol IV, Part B. Chicester: John Wiley \& Sons, 211-254.

Clément P, Wurdak E. 1991. Rotifera. In: Harrison FW, Ruppert EE, eds. Microscopic anatomy of invertebrates. Vol. 4. Aschelminthes. New York: Wiley-Liss, 219-297.

Cloney RA. 1990. Urochordata - Ascidiacea. In: Adiyodi KG, Adiyodi RG, eds. Reproductive biology of invertebrates. Vol IV, Part B. Chicester: John Wiley \& Sons, 391-451.

Cobb JLS. 1995. The nervous systems of Echinodermata: recent results and new approaches. In: Breidbach O, Kutsch $\mathrm{W}$, eds. The nervous system of invertebrates: an evolutionary and comparative approach. Basel: Birkhäuser Verlag, 407-424.

Cohen BL. 2000. Monophyly of brachiopods and phoronids: reconciliation of molecular evidence with Linnaean classification (the subphylum Phoroniformea nov.). Proc. R. Soc. Lond. B. 267: 225-231.

Cohen BL, Gawthorp A, Cavalier-Smith T. 1998. Molecular phylogeny of brachiopods and phoronids based on nuclearencoded small subunit ribosomal RNA gene sequences. Phil. Trans. R. Soc. Lond. B 353: 2039-2061.

Costello DP. 1955. Cleavage, blastulation and gastrulation. In: Willier BH, Weiss PA, Hamburger V, eds. Analysis of Development. Philadelphia: W. B. Saunders Company, 213-229.

Costello DP, Henley C. 1976. Spiralian development: a perspective. Amer. Zool. 16: 277-291.

Cragg SM. 1996. The phylogenetic significance of some anatomical features of bivalve veliger larvae. In: Taylor JD, ed. Origin and evolutionary radiation of the Mollusca. Oxford: Oxford University Press, 371-380.

Crompton DWT. 1989. Acanthocephala. In: Adiyodi KG, Adiyodi RG, eds. Reproductive biology of invertebrates. Vol $I V$, Part A. Chicester: John Wiley \& Sons, 251-258.

Cunha A, Azevedo RBR, Emmons SW, Leroi AM. 1999. Variable cell number in nematodes. Nature 402: 253.

Cunningham CW. 1997. Is congruence between data partitions a reliable predictor of phylogenetic accuracy? Empirically testing an iterative procedure for choosing among phylogenetic methods. Syst. Biol. 46: 464-478.

Damen P, Dictus WJAG. 1994. Cell lineage of the prototroch of Patella vulgata (Gastropoda, Mollusca). Dev. Biol. 162: 364-383.

Dautov SS, Nezlin LP. 1992. Nervous system of the tornaria larva (Hemichordata: Enteropneusta): a histochemical and ultrastructural study. Biol. Bull. 183: 463-475.

Davidson EH. 1991. Spatial mechanisms of gene regulation in metazoan embryos. Development 113: 1-26.

Davidson EH. 2001. Genomic regulatory systems. Development and evolution. San Diego: Academic Press.

Davidson EH, Cameron RA, Ransick A. 1998. Specification of cell fate in the sea urchin embryo: summary and some proposed mechanisms. Development 125: 3269-3290.

Davis FC. 1989. Echiura. In: Adiyodi KG, Adiyodi RG, eds. Reproductive biology of invertebrates. Vol IV, Part A. Chicester: John Wiley \& Sons, 349-381. 
Dayrat B, Tillier S. 2000. Taxon sampling, character sampling and systematics: how gradist presuppositions created additional ganglia in gastropod eutyneuran taxa. Zool. J. Linn. Soc. 129: 403-418.

Desmond A. 1994. Huxley. The devil's disciple. London: Michael Joseph.

Dewel RA. 2000. Colonial origin for Eumetazoa: major morphological transitions and the origin of bilaterian complexity. J. Morph. 243: 35-74.

Dewel RA, Dewel WC. 1996. The brain of Echiniscus viridissimus Peterfi, 1956 (Heterotardigrada): A key to understanding the phylogenetic position of tardigrades and the evolution of the arthropod head. Zool. J. Linn. Soc. 116: 35-49.

Dewel RA, Dewel WC. 1997. The place of tardigrades in arthropod evolution. In: Fortey RA, Thomas RH, eds. Arthropod relationships. London: The Systematics Association Special Volume Series 55, Chapman and Hall, 109-123.

Dewel RA, Nelson DR, Dewel WC. 1993. Tardigrada. In: Harrison FW, Rice ME, eds. Microscopic anatomy of invertebrates. Vol. 12. Onychophora, Chilopoda, and lesser Protostomata. New York: Wiley-Liss, 143-183.

Dewel RA, Budd GE, Castano DF, Dewel WC. 1999. The organization of the subesophageal nervous system in tardigrades: insights into the evolution of the arthropod hypostome and tritocerebrum. Zool. Anz. 238: 191-203.

Dickinson AJG, Croll RP, Voronezhskaya EE. 2000. Development of embryonic cells containing serotonin, catecholamines, and FMRFamide-related peptides in Aplysia californica. Biol. Bull. 199: 305-315.

Dictus WJAG, Damen P. 1997. Cell-lineage and clonal-contribution map of the trochophore larva of Patella vulgata (Mollusca). Mech. Dev. 62: 213-226.

Dorresteijn AWC, O'Grady B, Fischer A, Porchet-Henneré E, Boilly-MarerY. 1993. Molecular specification of cell lines in the embryo of Platynereis (Annelida). Roux's Arch. Dev. Biol. 202: 260-269.

Dunagan, TT, Miller DM. 1991. Acanthocephala. In: Harrison FW, Ruppert EE, eds. Microscopic anatomy of invertebrates. Vol. 4. Aschelminthes. New York: Wiley-Liss, 299-332.

Dzik J. 1993. Early metazoan evolution and the meaning of its fossil record. Evol. Biol. 27: 339-386.

Edgecombe GD, Wilson GDF, Colgan DJ, Gray MR, Cassis G. 2000. Arthropod cladistics: combined analysis of histone $\mathrm{H} 3$ and U2 snRNA sequences and morphology. Cladistics 16: $155-203$.

Eeckhaut 1. 1995. Cycle vital et biologie de Myzostoma cirriferum (Myzostomida), symbiote obligatoire de la comatule Antedon bifida (Echinodermata). Belgium: Ph.D thesis, Université de Mons-Hainaut.

Eeckhaut I, McHugh D, Mardulyn P, Tiedemann R, Monteyne D, Jangoux M, Milinkovitch MC. 2000 . Myzostomida: a link between trochozoans and flatworms? Proc. R. Soc. Lond. B. 267: 1383-1392.

Eernisse DJ. 1997. Arthropod and annelid relationships re-examined. In: Fortey RA, Thomas RH, eds. Arthropod relationships. London: The Systematics Association Special Volume Series 55, Chapman and Hall, 43-56.
Eernisse DJ, Albert JS, Anderson FE. 1992. Annelida and Arthropoda are not sister taxa: a phylogenetic analysis of spiralian metazoan morphology. Syst. Biol. 41: 305-330.

Eernisse DJ, Reynolds PD. 1994. Polyplacophora. In: Harrison FW, Kohn AJ, eds. Microscopic anatomy of invertebrates. Vol. 5. Mollusca I. New York: Wiley-Liss, 55-110.

Ehlers U. 1985. Das phylogenetische System der Plathelminthes. Stuttgart: Gustav Fischer Verlag.

Ehlers U. 1992. Frontal glandular and sensory structures in Nemertoderma (Nemertodermatida) and Paratomella (Acoela): ultrastructure and phylogenetic implications for the monophyly of the Euplathelminthes. Zoomorphology 112: 227-236.

Ehlers U. 1993. Ultrastructure of the spermatozoa of Halammohydra schulzei (Cnidaria, Hydrozoa): the significance of acrosomal structures for the systematization of the Eumetazoa. Microfauna Marina 8: 115-130.

Ehlers U. 1997. Ultrastructure of the statocysts in the apodous sea cucumber Leptosynapta inhaerens (Holothuroidea, Echinodermata). Acta Zool. 78: 61-68.

Ehlers U, Ahlrichs W, Lemburg C, Schmidt-Rhaesa A. 1996. Phylogenetic sy'stematization of the Nemathelminthes (Aschelminthes). Verhandlungen der Deutschen Zoologischen Gesellschafi 89, 8.

Ehlers U, Sopott-Ehlers B. 1997. Xenoturbella bocki: organization and phylogenetic position as sistertaxon of the Bilateria. Verh. D. Zool. Ges. 80: 189.

Eibye-Jacobsen J. 1996/1997. New observations on the embryology of the Tardigrada. Zool. Anz. 235: 201-216.

Ellis EHJr, Fausto-Sterling A. 1997. Platyhelminthes, the flatworms. In: Gilbert SF, Raunio AM, eds. Embryology. Constructing the organism. Sunderland: Sinauer Associates, 115-130.

Emig CC. 1982. The biology of Phoronida. Adv. Mar. Biol. 19: 1-89.

Emig CC. 1990. Phoronida. In: Adiyodi KG, Adiyodi RG, eds. Reproductive biology of invertebrates. Vol IV, Part B. Chicester: John Wiley \& Sons, 165-184.

Emschermann P. 1996. Kamptozoa (Entoprocta), Kelchwürmer. In: Westheide W, Rieger RM, eds. Spezielle Zoologie. Erster Teil: Einzeller und Wirbellose Tiere. Stuttgart: G. Fischer, 337-344.

Eriksson BJ, Budd GE. 2000. Onychophoran cephalic nerves and their bearing on our understanding of head segmentation and stem-group evolution of Arthropoda. Arthropod Struct. Dev. 29: 197-209.

Eriksson BJ, Tait NN, Budd GE. 2003. Head development in the onychophoran Euperipatoides kanangrensis with particular reference to the central nervous system. J. Morph. 255: $1-23$.

Erwin DH, Davidson EH. 2002. The last common bilaterian ancestor. Development 129: 3021-3032.

Fauchald K, Rouse G. 1997. Polychaete systematics: past and present. Zoologica Scripta 26: 71-138.

Fautin DG, Spaulding JG, Chia F-S. 1989. Cnidaria. In: Adiyodi KG, Adiyodi RG, eds. Reproductive biology of invertebrates. Vol IV, Part A. Chicester: John Wiley \& Sons, 43-62. 
Fautin DG, Marsical RN. 1991. Cnidaria: Anthozoa. In: Harrison FW, Westfall JA, eds. Microscopic anatomy of invertebrates. Vol. 2. Placozoa, Porifera, Cnidaria, and Ctenophora. New York: Wiley-Liss, 267-358.

Fell PE. 1989. Porifera. In: Adiyodi KG, Adiyodi RG, eds. Reproductive biology of invertebrates. Vol IV, Part A. Chicester: John Wiley \& Sons, 1-41.

Fell PE. 1997. Poriferans, the sponges. In: Gilbert SF, Raunio AM, eds. Embryology. Constructing the organism. Sunderland: Sinauer Associates, 39-54.

Fenaux R. 1998. Anatomy and functional morphology of the Appendicularia. In: Bone Q, ed. Biology of pelagic tunicates. New York: Oxford University Press, 25-34.

Fernández J, Téllez V, Olea N. 1992. Hirudinea. In: Harrison FW, Gardiner SL, eds. Microscopic anatomy of invertebrates. Vol. 7. Annelida. New York: Wiley-Liss, 323-394.

Ficld KG, Olsen G, Lane DJ, Giovannoni SJ, Ghiselin MT, Raff EC, Pace NR, Raff RA. 1988. Molecular phylogeny of the animal kingdom. Science 239: 748-753.

Fischer A. 1999. Reproductive and developmental phenomena in annelids: a source of examplary research problems. Hydrobiologia 402: 1-20.

Flügel HJ, Callsen-Cencic P. 1992. New observations on the biology of Siboglinum poseidoni Flügel \& Langhof (Pogonophora) from the Skagerrak. Sarsia 77: 287-290.

Forey PL, Kitching IJ. 2000. Experiments in coding multistate characters. In: Scotland R, Pennington RT, eds. Homology and systematics. Coding characters for phylogenetic analysis. London: Taylor \& Francis, 54-80.

Fortey RA, Thomas RH. 1997. Arthropod relationships. London: Chapman \& Hall.

Franke M. 1993. Ultrastructure of the protonephridia in Loxosomella fauveli, Barentsia matsushimana and Pedicellina cernua. Implications for the protonephridia in the ground pattern of the Entoprocta (Kamptozoa). Microfauna Marina 8: 7-38.

Fransen ME. 1988. Coelomic and vascular systems. In: Westheide W, Hermans CO, eds. The ultrastructure of Polychaeta. Stuttgart: Gustav Fischer Verlag, 199-213.

Franzén $\AA$. 1956. On spermiogenesis, morphology of the spermatozoon, and biology of fertilization among invertebrates. Zool. Bidrag Från Uppsala 31: 355-482.

Franzén A. 1983a. Nemertina. In: Adiyodi KG, Adiyodi RG, eds. Reproductive biology of invertebrates. Chicester: John Wiley \& Sons, 159-170.

Franzén Å. 1983b. Bryozoa Entoprocta. In: Adiyodi KG, Adiyodi RG, eds. Reproductive biology of invertebrates. Chicester: John Wiley \& Sons, 505-516.

Franzén Å, Rice SA. 1988. Spermatogenesis, male gametes and gamete interactions. In: Westheide $\mathrm{W}$, Hermans $\mathrm{CO}$, eds. The ultrastructure of Polychaeta. Stuttgart; Gustav Fischer Verlag, 309-333.

Freeman G. 1993. Regional specification during embryogenesis in the articulate brachiopod Terebratalia. Dev. Biol. 160: 196-213.

Freeman G. 1995. Regional specification during embryogenesis in the inarticulate brachiopod Glottidia. Dev. Biol. 172: 15-36.
Freeman G. 1999. Regional specification during embryogenesis in the inarticulate brachiopod Discinisca. Dev. Biol. 209: 321-339.

Freeman G. 2000. Regional specificattion during embryogenesis in the craniiform brachiopod Crania anomala. Dev. Biol. 227: 219-238.

Freeman G, Martindale MQ. 2002. The origin of mesoderm in phoronids. Dev. Biol. 252: 301-311.

Friedrich S, Wanninger A, Brückner M, Haszprunar G. 2002. Neurogenesis in the mossy chiton, Mopalia muscosa (Gould) (Polyplacophora): evidence against molluscan metamerism. J. Morph. 253: 109-117.

Funch P. 1996. The chordoid larva of Symbion pandora (Cycliophora) is a modified trochophore. J. Morph. 230: 231263.

Funch P, Kristensen RM. 1997. Cycliophora. In: Harrison FW, Woollacott RM, eds. Microscopic anatomy of invertebrates. Lophophorates, Entoprocta, and Cycliophora. New York: Wiley-Liss, 409-474.

Furuya H, Tsuneki K, Khoshida Y. 1992. Development of the infusoriform embryo of Dicyema japonicum (Mesozoa: Dicyemidae). Biol. Bull. 183: 248-257.

Galleni L, Gremigni V. 1989. Platyhelminthes. Turbellaria. In: Adiyodi KG, Adiyodi RG, eds. Reproductive biology of invertebrates. Vol. IV, Part A. New York: John Wiley \& Sons, 63-89.

Galt CP, Fenaux R. 1990. Urochordata - Larvacea. In: Adiyodi KG, Adiyodi RG, eds. Reproductive biology of invertebrates. Vol IV, Part B. Chicester: John Wiley \& Sons, 471-500.

García-Varela M, Pérez-Ponce de León G, De la Torre P, Cummings MP, Sarma SSS, Laclette JP. 2000. Phylogenetic relationships of Acanthocephala based on analysis of 18 S ribosomal RNA gene sequences. L. Mol. Evol. 50: 532540.

García-Varela M, Cummings MP, Pérez-Ponce de León G, Gardner SL, Laclette JP. 2002. Phylogenetic analysis based on 18S ribosomal RNA gene sequences supports the existence of class Polyacanthocephala (Acanthocephala). Mol. Phyl. Evol. 23: 288-292.

Gardiner SL, Jones ML. 1993. Vestimentifera. In: Harrison FW, Rice ME, eds. Microscopic anatomy of invertebrates. Vol. 12. Onychophora, Chilopoda, and lesser Protostomata. New York: Wiley-Liss, 371-460.

Gardiner SL, Jones ML. 1994. On the significance of larval and juvenile morphology for suggesting phylogenetic relationships of the Vestimentifera. Amer. Zool. 34: 513-522.

Garey, JR, Near TJ, Nonnemacher MR, Nadler SA. 1996. Molecular evidence for Acanthocephala as a subtaxon of Rotifera. J. Mol. Evol. 43: 287-292.

Garey JR, Schmidt-Rhaesa A. 1998. The essential role of "minor" phyla in molecular studies of animal evolution. Amer. Zool. 38: 907-917.

Garey JR, Schmidt-Rhaesa A, Near TJ, Nadler SA. 1998. The evolutionary relationships of rotifers and acanthocephalans. Hydrobiologia 387/388: 83-91.

Gerberding M, Browne WE, Patel NH. 2002. Cell lineage analysis of the amphipod crustacean Parhyale hawaiensis reveals an early restriction of cell fates. Development 129: 5789-5801. 
Gerhart J. 2000. Inversion of the chordate body axis: are there alternatives? Proc. Natl. Acad. Sci. USA.97: 4445-4448.

Ghiara G. 1995. Structure of the early vertebrate gastrula and causal factors of morphogenesis and differentiation: a critical survey of the hundred year history of ideas. In: Lanzavecchia G, Valvassori R, Candia Carnevali MD, eds. Body cavities: function and phylogeny. Modena: Mucchi, 199-232.

Ghirardelli E. 1995. Chaetognaths: two unsolved problems: the coelom and their affinities. In: Lanzavecchia G, Valvassori $\mathrm{R}$, Candia Carnevali MD, eds. Body cavities: function and phylogeny. Modena: Mucchi, 167-185.

Ghiselin MT. 1969. The evolution of hermaphroditism among animals. Quart. Rev. Biol. 44: 189-208.

Ghiselin MT. 1989. Summary of our present knowledge of metazoan phylogeny. In: Fernholm B, Bremer K, Jörnvall H, eds. The Hierarchy of life. Molecules and Morphology in phylogenetic analysis. Amsterdam: Proceedings from Nobel Symposium 70, 1988. Excerpta Medica/Elsevier, 261-272.

Gilbert JJ. 1989. Rotifera. In: Adiyodi KG, Adiyodi RG, eds. Reproductive biology of invertebrates. Vol IV, Part A. Chicester: John Wiley \& Sons, 179-199.

Gilbert SF, Raunio AM. 1997. Embryology. Constructing the organism. Sunderland: Sinauer Associates.

Gilmour THJ. 1978. Ciliation and function of the food-collecting and waste-rejecting organs of lophophorates. Can. J. Zool. 56: 2142-2155.

Gilmour THJ. 1981. Food-collecting and waste-rejecting mechanisms in Glottidia pyramidata and the persistence of lingulacean inarticulate brachiopods in the fossil record. Can. J. Zool. 59: 1539-1547.

Giribet G. 1999. Ecdysozoa versus Articulata, dos hipótesis alternativas sobre la posición de los artrópodos en el reino animal. Boletin S. E. A. 26: 145-160.

Giribet G. 2002. Current advances in the phylogenetic reconstruction of metazoan evolution. A new paradigm for the Cambrian explosion? Mol. Phyl. Evol. 24: 345-357.

Giribet G, Ribera C. 1998. The position of arthropods in the animal kingdom: a search for a reliable outgroup for internal arthropod phylogeny. Mol. Phyl. Evol. 9: 481-488.

Giribet G, Ribera C. 2000. A review of arthropod phylogeny: new data based on ribosomal DNA sequences and direct character optimization. Cladistics 16: 204-231.

Giribet G, Wheeler WC. 1999. The position of arthropods in the animal kingdom: ecdysozoa, islands, trees, and the "parsimony ratchet." Mol. Phyl. Evol. 13: 619-623.

Giribet G, Distel DL, Polz M, Sterrer W, Wheeler WC. 2000. Triploblastic relationships with emphasis on the acoelomates and the position of Gnathostomulida, Cycliophora, Plathelminthes, and Chaetognatha: a combined approach of $18 \mathrm{~S}$ rDNA sequences and morphology. Syst. Biol. 49: 539-562.

Giribet G, Edgecombe GD, Wheeler WC. 2001. Arthropod phylogeny based on eight molecular loci and morphology. Nature 413: 157-161.

Giribet, G, Edgecombe, GD, Wheeler WC, Babbitt C. 2002. Phylogeny and systematic position of Opiliones: a combined analysis of chelicerate relationships using morphological and molecular data. Cladistics 18: 5-70.

Glasby CJ, Hutchings PA, Fauchald K, Paxton HH, Rouse
GW, Watson Russell C, Wilson RS. 2000. Class Polychaeta. In: Beesley PL, Ross GJB, Glasby CJ, eds. Polychaetes and allies. The Southern synthesis. Fauna of Australia. Volume 4 A Polychaeta, Myzostomida, Pogonophora, Echiura, Sipunculida. CSIRO Collingwood: Publishing, 1-296.

Godeaux JEA. 1990. Urochordata - Thaliacea. In: Adiyodi KG, Adiyodi RG, eds. Reproductive biology of invertebrates. Vol IV, Part B. Chicester: John Wiley \& Sons, 453-469.

Golding DW. 1992. Polychaeta: nervous system. In: Harrison FW, Gardiner S1, eds. Microscopic anatomy of invertebrates. Vol. 7. Annelida. New York: Wiley-Liss, 153-179.

Goldschmid A. 1996a. Echinodermata, Stachelhäuter. In: Westheide W, Rieger RM, eds. Spezielle Zoologie. ErsterTeil: Einzeller und Wirbellose Tiere. Stuttgart: Gustav Fischer Verlag, 778-834.

Goldschmid A. 1996b. Hemichordata, Branchiotremata. In: Westheide W, Rieger RM, eds. Spezielle Zoologie. Erster Teil: Einzeller und Wirbellose Tiere. Stuttgart: Gustav Fischer Verlag, 763-777.

Goldstein B, Freeman G. 1997. Axis specification in animal development. BioEssays 19: 105-116.

Goodrich ES. 1895. On the coelom, genital ducts, and nephridia. Quart. J. Micros. Sci. 37: .477-510 + two plates.

Goodrich ES. 1958. Studies on the structure and development of vertebrates. New York: Dover Publications, Inc.

Götting K-J. 1996. Mollusca, Weichtiere. In: Westheide W, Rieger RM, eds. Spezielle Zoologie. Erster Teil: Einzeller und Wirbellose Tiere. Stuttgart: Gustav Fischer Verlag, 276330.

Gould SJ. 1986. Geoffroy and the homeobox. In: Slavkin HC, ed. Progress in developmental biology, part $A$. New York: Alan R. Liss, Inc. 205-218.

Green CR, Bergquist PR. 1982. Phylogenetic relationships within the Invertebrata in relation to the structure of septate junctions and the development of "occluding" junctional types. J. Cell Sci. 53: 279-305.

Grell KG, Ruthmann A. 1991. Placozoa. In: Harrison FW, Westfall JA, eds. Microscopic anatomy of invertebrates. Vol. 2. Placozoa, Porifera, Cnidaria, and Ctenophora. New York: Wiley-Liss, 13-27.

Grimmelikhuijzen CJP, Westfall, JA. 1995. The nervous system of cnidarians. In: Breidbach $\mathrm{O}$, Kutsch $\mathrm{W}$, eds. The nervous system of invertebrates: an evolutionary and comparative approach. Basel: Birkhäuser Verlag, 7-24.

Grobe P. 2000. The ultrastructure of the larva of Neocrania anomala (Brachiopoda). Zoology 103 (Supplement III): 101.

Grygier MJ. 2000. Class Myzostomida. In: Beesley PL, Ross GJB, Glasby CJ, eds. Polychaetes and allies. The Southern synthesis. Fauna of Australia. Volume 4A Polychaeta, Myzostomida, Pogonophora, Echiura, Sipunculida. Collingwood: CSIRO Publishing, 297-329.

Gschwentner R, Ladurner P, Nimeth K, Rieger R. 2001. Stem cells in a basal bilaterian. S-phase and mitotic cells in Convolutriloba longifissura (Acoela, Platyhelminthes). Cell Tissue Res. 304: 401-408.

Guralnick RP, Lindberg DR. 2001. Reconnecting cell and animal lineages: what do cell lineages tell us about the evolution and development of Spiralia? Evolution 55: 1501-1519. 
Guralnick RP, Lindberg DR. 2002. Cell lineage data and spiralian evolution: a reply to Nielsen and Meier. Evolution 56: 2558-2560.

Hadfield MG. 1975. Hemichordata. In: Giese AC, Pearse JS, eds. Entoprocts and lesser coelomates. New York: Academic Press, 185-240.

Hadfield MG, Strathmann MF, Strathmann RR. 1997. Ciliary currents of non-feeding veligers in putative basal clades of gastropods. Invert. Biol. 116: 313-321.

Hadzi J. 1953. An attempt to reconstruct the system of animal classification. Syst. Zool 2: 145-154.

Ilalanych KM. 1995. The phylogenetic position of the pterobranch hemichordates based on 18S rDNA sequence data. Mol. Phyl. Evol. 4: 72-76.

IIalanych KM. 1998. Considerations for reconstructing metazoan history: signal, resolution, and hypothesis testing. Amer. Zool. 38: 929-941.

Halanych KM, Feldman RA, Vrijenhoek RC. 2001. Molecular evidence that Sclerolinum brattstromi is closely related to vestimentiferans, not to frenulate pogonophorans (Siboglinidae, Annelida). Biol. Bull. 201: 65-75.

Hall BK. 1998. Germ layers and the germ-layer theory revisited. Primary and secondary germ layers, neural crest as a fourth germ layer, homology, and demise of the germ-layer theory. Evol. Biol. 30: 121-186.

I Iall BK. 1999. Evolutionary developmental biology. Second edition. Dordrecht: Kluwer Academic Publishers.

Hammersten OD, Runnström J. 1925. Zur Embryologie von Acanthochiton discrepans Brown. Zool. Jb. Anat. 47: 261318.

Harrison FW, De Vos L. 1991. Porifera. In: Harrison FW, Westfall JA, eds. Microscopic anatomy of invertebrates. Vol. 2. Placozoa, Porifera, Cnidaria, and Ctenophora. New York: Wiley-Liss, 29-89.

IIartenstein V, Ehlers U. 2000. The embryonic development of the rhabdocoel flatworm Mesostoma lingua (Abildgaard, 1798). Dev. Genes Evol. 210: 399-415.

Haszprunar G. 1988. On the origin and evolution of major gastropod groups, with special reference to the Streptoneura. J. Moll. Stud. 54: 367-441.

Haszprunar G. 1992. The first molluscs - small animals. Boll. Zool. 59: 1-16.

Haszprunar G. 1996a. The Mollusca: coelomate turbellarians or mesenchymate annelids? In: Taylor JD, ed. Origin and evolutionary radiation of the Mollusca. Oxford: Oxford University Press, 1-28.

Haszprunar G. 1996b. Plathelminthes and Plathelminthomorpha - paraphyletic taxa. J. Zool. Syst. Evol. Res. 34: 4148.

Haszprunar G. 1996c. "Mesozoa". In: Westheide W, Rieger, RM, eds. Spezielle Zoologie. Erster Teil: Einzeller und Wirbellose Tiere. Stuttgart: Gustav Fischer Verlag, 125-129.

Haszprunar G. 1996d. The molluscan rhogocyte (pore-cell, Blasenzelle, cellule nucale), and its significance for ideas on nephridial evolution. J. Moll. Stud. 62: 185-211.

Haszprunar G. 1997. Ultrastructure of the pseudo-protonephrium of the enigmatic opisthobranch, Rhodope transtrosa (Gastropoda, Nudibranchia). J. Submicros. Cytol. Pathol. 29: $371-378$.
Haszprunar G. 2000. Is the Aplacophora monophyletic? A cladistic point of view. Amer. Malac. Bull. 15: 115-130.

Haszprunar G, Salvini-Plawen L von, Rieger RM. 1995. Larval planktotrophy-a primitive trait in the Bilateria? Acta Zool. 76: 141-154.

Haszprunar G, Schaefer K. 1997. Monoplacophora. In: Harrison FW, Kohn AJ, eds. Microscopic anatomy of invertebrates. Vol. 6B. Mollusca II. New York: Wiley-Liss, 415457.

Haszprunar G, Ruthensteiner B. 2000. Microanatomy and ultrastructure of the protonephridial system in the larva of the limpet, Patella vulgata L. (Mollusca, Patello-gastropoda). J. Submicros. Cytol. Pathol. 32: 59-67.

Haszprunar G, Wanninger A. 2000. Molluscan muscle systems in development and evolution. J. Zool. Syst. Evol. Res. 38: 157-163.

Hatschek B. 1911. Das neue zoologische System. Leipzig: Verlag von Wilhelm Engelmann.

Hausdorf B. 2000. Early evolution of the Bilateria. Syst. Biol.49: 130-142.

Hawkins JA. 2000. A survey of primary homology assessment: different botanists perceive and define characters in different ways. In: Scotland R. Pennington RT, eds. Homology and systematics. Coding characters for phylogenetic analysis. London: Taylor \& Francis, 22-53.

Hawkins JA, Hughes CE, Scotland RW. 1997. Primary homology assessment, characters and character states. Cladistics 13: 275-283.

Hay-Schmidt A. 1990. Catecholamine-containing, serotoninlike and neuropeptide FMRFamide-like immunoreactive cells and processes in the nervous system of the pilidium larva (Nemertini). Zoomorphology 109: 231-244.

Hay-Schmidt A. 1992. Ultrastructure and immunocytochemistry of the nervous system of the larvae of Lingula anatina and Glottidia sp. (Brachiopoda). Zoomorphology 112: 189205.

Hay-Schmidt A. 2000. The evolution of the serotonergic nervous system. Proc. R. Soc. Lond. B 267: 1071-1079.

Heimler W. 1988. Larvae. In: Westheide W, Hermans Co, eds.

I The ultrastructure of Polychaeta. Stuttgart: Gustav Fischer Verlag, 353-371.

Heinzeller T, Welsch U. 1994. Crinoidea. In: Harrison FW, Chia F-S, eds. Microscopic anatomy of invertebrates. Vol. 14. Echinodermata. New York: Wiley-Liss, 9-148.

Hendelberg J. 1983. Platyhelminthes - Turbellaria. In: Adiyodi KG, Adiyodi RG, eds. Reproductive biology of invertebrates. Chicester: John Wiley \& Sons, 75-104.

Hendler G. 1991. Echinodermata: Ophiuroidea. In: Giese AC, Pearse JS, Pearse VB, eds. Reproduction of marine invertebrates. Vol. VI. Echinoderms and lophophorates. California: The Boxwood Press, 356-511.

Henry J, Martindale MQ. 1997. Nemerteans, the ribbon worms. In: Gilbert SF, Raunio AM, eds. Embryology. Constructing the organism. Sunderland: Sinauer Associates, 151166.

Henry JJ, Martindale MQ. 1998a. Conservation of the spiralian developmental program: cell lineage of the nemertean, Cerebratulus lacteus. Dev. Biol. 201: 253-269. 
Henry JQ, Martindale MQ. 1998b. Evolution of cleavage programs in relationship to axial specification and body plan evolution. Biol. Bull. 195: 363-366.

Henry JJ, Martindale MQ. 1999. Conservation and innovation in spiralian development. Hydrobiologia 402: 255-265.

Henry JQ, Martindale MQ, Boyer BC. 2000. The unique developmental program of the acoel flatworm, Neochildia fusca. Dev. Biol. 220: 285-295.

Herlyn H, Ehlers U. 1997. Ultrastructure and function of the pharynx of Gnathostomula paradoxa (Gnathostomulida). Zoomorphology 117: 135-145.

Herlyn H, Piskurek O, Schmitz J, Ehlers U, Zischler H. 2003. The syndermatan phylogeny and the evolution of acanthocephalan endoparasitism as inferred from 18S rDNA sequences. Mol. Phyl. Evol. 26: 155-164.

Hernandez-Nicaise M-L. 1991. Ctenophora. In: Harrison FW, Westfall JA, eds. Microscopic anatomy of invertebrates. Vol. 2. Placozoa, Porifera, Cnidaria, and Ctenophora. New York: Wiley-Liss, 359-418.

Herrmann K. 1996. Phoronida, Hufeisenwürmer. In: Westheide W, Rieger RM, eds. Spezielle Zoologie. Erster Teil: Einzeller und Wirbellose Tiere. Stuttgart: Gustav Fischer Verlag, 740742.

Herrmann K. 1997. Phoronida. In: Harrison FW, Woollacott RM, eds. Microscopic anatomy of invertebrates. Vol. 13. Lophophorates, Entoprocta, and Cycliophora. New York: WileyLiss, 207-235.

Hertzler PL. 2002. Development of the mesendoderm in the dendrobranchiate shrimp Siccyonia ingentis. Arthr. Struct. Devel. 31: 33-49.

Hessler RH, Elofsson R. 1995. Segmental podocyte excretory glands in the thorax of Hutchinsoniella macracantha (Cephalocarida). J. Crust. Biol. 15: 61-9.

Hessling R. 2002. Metameric organisation of the nervous system in developmental stages of Urechis caupo (Echiura) and its phylogenetic implications. Zoomorphology 121: 221-234.

Hessling R, Westheide W. 2002. Are Echiura derived from a segmented ancestor? Immunocytochemical analysis of the nervous system in developmental stages of Bonellia viridis. J. Morph. 252: 100-113.

Hill SD, Boyer BC. 2001. Phalloidin labeling of developing muscle in embryos of the polychaete Capitella sp. I. Biol. Bull. 201: 257-258.

Hochberg R, Litvaitis MK. 2000. Phylogeny of Gastrotricha: a morphology-based framework of gastrotrich relationships. Biol. Bull. 198: 299-305.

Hochberg R, Litvaitis MK. 2001a. Macrodasyida (Gastrotricha): a cladistic analysis of morphology. Invert. Biol. 120: 124-135.

Hochberg R, Litvaitis MK. 2001b. The musculature of Draculiciteria tessalata (Chaetonotida, Paucitubulatina): implications for the evolution of dorsoventral muscles in Gastrotricha. Hydrobiologia 452: 155-161.

Holde KE van 1997. Respiratory proteins of invertebrates: Structure, function and evolution. Zool.-Anal. Complex Syst. 100: 287-297.

Holde KE van, Miller KI, Decker H. 2001. Hemocyanins and invertebrate evolution. J. Biol. Chem. 276: 15563-15566.
Holland ND. 1991. Echinodermata: Crinoidea. In: Giese AC, Pearse JS, Pearse VB, eds. Reproduction of marine invertebrates. Vol. VI. Echinoderms and lophophorates. California: The Boxwood Press, 247-299.

Holland ND, Holland LZ. 1999. Amphioxus and the utility of molecular genetic data for hypothesizing body part homologies between distantly related animals. Amer: Zool. 39: 630640.

Holland LZ, Kene M, Williams NA, Holland ND. 1997. Sequence and embryonic expression of the amphioxus engrailed gene (AmphiEn): the metameric pattern of transcription resembles that of its segment-polarity homolog in Drosophila. Development 124: 1723-1732.

Holland LZ, Holland ND. 1998. Developmental gene expression in amphioxus: new insights into the evolutionary origin of vertebrate brain regions, neural crest, and rostrocaudal segmentation. Amer. Zool. 38: 647-658.

Holley SA, Ferguson EL. 1997. Fish are like flies are like frogs: conservation of dorsal-ventral patterning mechanisms. BioEssays 19: 281-284.

Hörstadius S. 1937. Experiments on determination in the early development of Cerebratulus lacteus. Biol. Bull. 73: 317 342 .

Hotchkiss FHC. 1998. A "rays-as-appendages" model for the origin of pentamerism in echinoderms. Paleobiology 24: 200214.

Huxley TH. 1888. A manual of the anatomy of invertebrated animals. New York: D. Appleton and Company.

Huxley TH. 1859. On the agamic reproduction and morphology of Aphis. - Part I. Trans. Linn. Soc. 22: 193-219.

Hyman LH. 1940. The Invertebrates, vol. I. Protozoa through Ctenophora. New York: McGraw-Hill Book Company.

Hyman LH. 1951a. The Invertebrates, vol. II. Platyhelminthes and Rhynchocoela. The acoelomate Bilateria. New York: McGraw-Hill Book Company.

Hyman LH. 1951b. The invertebrates, vol. III. Acanthocephala, Aschelminthes and Entoprocta. New York: McGraw-Hill Book Company.

Hyman LH. 1955. The invertebrates, vol. IV. Echinodermata. New York: McGraw-Hill Book Company.

Hyman LH. 1959. The Invertebrates, vol. V. Smaller coelomate groups. Chaetognatha, Hemichordata, Pogonophora, Phoronida, Ectoprocta, Brachiopoda, Sipunculida. The coelomate Bilateria. New York: McGraw-Hill Book Company.

Hyman LH. 1967. The Invertebrates, vol. VI. Mollusca I. New York: McGraw-Hill Book Company.

Inglis WG. 1985. Evolutionary waves: patterns in the origins of animal phyla. Austr. J. Zool. 33: 153-178.

Israelsson O. 2000. Xenoturbella. Unpublished $\mathrm{PhD}$ thesis Stockholm: Stockholm University.

Ivanov AV. 1988. Analysis of the embryonic development of Pogonophora in connection with the problems of phylogenetics. Z. zool. Syst. Evolutionsforsch. 26: 161-185.

Iwata F. 1960. Studies on the comparative embryology of nemerteans with special reference to their interrelationships. Publ. Akkeshi Mar. Biol. Station 10: 1-51.

Iwata F. 1968. Nemertini. In: Kumé M, Dan K, eds. Invertebrate embryology. Belgrade: NOLIT Publishing House, 144158. 
Jägersten G. 1939. Zur Kenntniss der Larven entwicklung bei Myzostomum. Ark. Zool. 31: 1-21.

Jägersten G. 1972. Evolution of the metazoan life cycle. A comprehensive theory. London: Academic Press.

James MA. 1997. Brachiopoda: internal anatomy, embryology, and development. In: Harrison FW, Woollacott RM, eds. Microscopic anatomy of invertebrates. Vol. 13. Lophophorates, Entoprocta, and Cycliophora. New York: Wiley-Liss, 297-407.

Jamieson BGM. 1987. The ultrastructure and phylogeny of insect spermatozoa. Cambridge: Cambridge University Press.

Jamieson BGM. 1988. On the phylogeny and higher classification of the Oligochaeta. Cladistics 4: 367-410.

Jamieson BGM. 1991. Ultrastructure and phylogeny of crustacean spermatozoa. Mem. Queensland Mus. 31: 109-142.

Jamieson BGM. 1992. Oligochaeta. In: Harrison FW, Gardiner SL, eds. Microscopic anatomy of invertebrates. Vol. 7. Annelida. New York: Wiley-Liss, 217-322.

Janies D. 2001. Phylogenetic relationships of extant echinoderm classes. Can. J. Zool. 79: 1232-1250.

Janies D, DeSalle R. 1999. Development, evolution, and corroboration. Anat. Rec. 257: 6-14.

Jenner RA. 1999. Metazoan phylogeny as a tool in evolutionary biology: current problems and discrepancies in application. Belg. J. Zool. 129: 245-261.

Jenner RA. 2000. Evolution of animal body plans: the role of metazoan phylogeny at the interface between pattern and process. Evol. Devel. 2: 208-221.

Jenner RA. 2001a. Bilaterian phylogeny and uncritical recycling of morphological data sets. Syst. Biol. 50: 730-742.

Jenner RA. 2001b. Carrying metazoan phylogenetics forward in the $21^{\text {st }}$ century, Contr. Zool. 70: 181-184.

Jenner RA. 2002. Boolean logic and character state identity: pitfalls of character coding in metazoan cladistics. Contr. Zool. 71: 67-91.

Jenner RA. in press. Unleashing the force of cladistics? Metazoan phylogenetics and hypothesis testing. Integ. Comp. Biol.

Jenner RA, Schram FR. 1999. The grand game of metazoan phylogeny: rules and strategies. Biol. Rev. 74: 121-142.

Jenner RA, Schram FR. 2002. Systematic zoology: invertebrates. In: Minelli A, Contrafatto G, eds. Biological systematics, Encyclopedia of Life Support Systems (EOLSS). Oxford: EOLSS Publishers, [http://www.eolss.net].

Joffe BI, Kornakova EE. 2001. Flatworm phylogeneticist: between molecular hammer and morphological anvil. In: Littlewood DTJ, Bray RA, eds. Interrelationships of the Platyhelminthes. London: Taylor \& Francis, 279-291.

Joly R, Descamps M. 1987. Histology and ultrastructure of the myriapod brain. In: Gupta AP, ed. Arthropod brain. Its evolution, development, structure, and functions. New York: John Wiley \& Sons, Inc., 135-157.

Jondelius U, Ruiz-Trillo I, Baguñà J, Riutort M. 2002. The Nemertodermatida are basal bilaterians and not members of the Platyhelminthes. Zool. Scr. 31: 201-215.

Kapp H. 1996. Chaetognatha, Pfeilwürmer. In: Westheide W, Rieger RM, eds. Spezielle Zoologie. Erster Teil: Einzeller und Wirbellose Tiere. Stuttgart: Gustav Fischer Verlag, 757762 .
Kapp H. 2000. The unique embryology of Chaetognatha. Zool. Anz. 239: 263-266.

Katayama T, Wada H, Furuya H, Satoh N, Yamamoto $M$. 1995. Phylogenetic position of the dicyemid mesozoa inferred from 18S rDNA sequences. Biol. Bull. 189: 81-90.

Kempf SC, Page LR, Pires A. 1997. Development of serotonin-like immunoreactivity in the embryos and larvae of nudibranch mollusks with emphasis on the structure and possible function of the apical sensory organ. J. Comp. Neurol. 386: 507-528.

Kessler DS, Melton DA. 1994. Vertebrate embryonic induction: mesodermal and neural patterning. Science 266: 596603.

Kimmel CB. 1996. Was Urbilateria segmented? TIG 12: 329331 .

Kinchin IM. 1994. The biology of tardigrades. London: Portland Press.

Kleinow W. 1993. Biochemical studies on Brachionus plicatilis - hydrolytic enzymes, integument proteins and composition of trophi. Hydrobiologia 255: 1-12.

Klerkx JHEM. 2001. Molecular analysis of early specification in the mollusc Patella vulgata. Unpublished $\mathrm{PhD}$ thesis. Utrecht: Universiteit Utrecht.

Kluge AG. 1997. Testability and the refutation and corroboration of cladistic hypotheses. Cladistics 13: 81-96.

Kluge AG. 1998. Sophisticated falsification and research cycles: consequences for differential character weighting in phylogenetic systematics. Zool. Scr. 26: 349-360.

Knauss E. 1979. Indication of an anal pore in Gnathostomulida. Zool. Scr. 8: 181-186.

Knoll AH, Carroll SB. 1999. Early animal evolution: emerging views from comparative biology and geology. Science 284: 2129-2137.

Kobayashi M, Furuya H, Holland PWH. 1999. Dicyemids are higher animals. Nature 401: 762.

Komai T. 1968. Ctenophora. In: Kumé M, Dan K, eds. Invertebrate embryology. Belgrade: NOLIT Publishing House, 117. 124.

Korn H. 1960. Ergänzende Beobachtungen zur Struktur der

Larve von Echiurus abyssalis. Skor. Z. wiss. Zool. 164: 199237.

Kowalevsky A. 1882. Weitere Studien über die Entwicklung der Chitonen. Zool. Anz. 5: 307-310.

Kozloff EN. 1990. Invertebrates. New York: Saunders College Publishing.

Kristensen RM. 1991a. Loricifera. In: Harrison FW, Ruppert EE, eds. Microscopic anatomy of invertebrates. Vol. 4. Aschelminthes. New York: Wiley-Liss, 351-375.

Kristensen RM. 1991b. Loricifera - a general biological and phylogenetic overview. Verh. Deutsch. Zool. Gesell. 84: 231246.

Kristensen RM. 1995. Are Aschelminthes pseudocoelomate or acoelomate? In: Lanzavecchia G, Valvassori R, Candia Carnevali MD, eds. Body cavities: function and phylogeny. Modena: Mucchi, 41-43.

Kristensen RM, Higgins RP. 1991. Kinorhyncha. In: Harrison FW, Ruppert EE, eds. Microscopic anatomy of invertebrates. Vol. 4. Aschelminthes. New York: Wiley-Liss, 377-404. 
Kristensen RM, Funch P. 2000. Micrognathozoa: a new class with complicated jaws like those of Rotifera and Gnathostomulida. J. Morph. 246: 1-49.

Kristensen RM, Brooke S. 2002. Phylum Loricifera. In: Young $\mathrm{CM}$, Sewell MA, Rice ME, eds. Atlas of marine invertebrate larvae. New York: Academic Press, 179-187.

Kristensen RM, Funch P. 2002. Phylum Cycliophora. In: Young $\mathrm{CM}$, Sewell MA, Rice ME, eds. Atlas of marine invertebrate larvae. New York: Academic Press, 199-208.

Kumé M. 1968a. Brachiopoda. In: Kumé M, Dan K, eds. Invertebrate embryology. Belgrade: NOLIT Publishing House, 269-279.

Kumé M. 1968b. Phoronidea. In: Kumé M, Dan K, eds. Invertebrate embryology. Belgrade: NOLIT Publishing House, 242-249.

Kupriyanova EK, Nishi E, Ten Hove HA, Rzhavsky AV. 2001. Life-history patterns in serpulimorph polychaetes: ecological and evolutionary perspectives. Oceanogr. Mar. Biol. 39: $1-101$.

Kusche K, Ruhberg H, Burmester T. 2002. A hemocyanin from the Onychophora and the emergence of respiratory proteins. Proc. Natl. Acad. Sci. USA 99: 10545-10548.

Lacalli TC. 1982. The nervous system and ciliary band of Müller's larva. Proc. R. Soc. Lond. B 217: 37-58.

Lacalli TC. 1983. The brain and central nervous system of Müller's larva. Can. J. Zool. 61: 39-51.

Lacalli TC. 1984. Structure and organization of the nervous system in the trochophore larva of Spirobranchus. Phil. Trans. R. Soc. Lond. B 306: 79-135.

Lacalli TC. 1994. Apical organs, epithelial domains, and the origin of the chordate central nervous system. Amer. Zool. 34: 533-541.

Lacalli TC. 1996a. Landmarks and subdomains in the larval brain of Branchiostoma: vertebrate homologs and invertebrate antecedents. Isr. J. Zool. 42: S131-S146.

Lacalli TC. 1996b. Mesodermal pattern and pattern repeats in the starfish bipinnaria larva, and related patterns in other deuterostome larvae and chordates. Phil. Trans. R. Soc. Lond. $B$ 351: $1737-1758$.

Lacalli TC. 1996c. Frontal eye circuitry, rostral sensory pathways and brain organization in amphioxus larvae: evidence from 3D reconstructions. Phil. Trans. $R$. Soc. Lond. B 351: 243-263.

Lacalli TC.1996d. Dorsoventral axis inversion: a phylogenetic perspective. BioEssays 18: 251-254.

Lacalli TC. 1997. The nature and origin of deuterostomes: some unresolved issues. Invert. Biol. 116: 363-370.

Lacalli TC. 1999. Tunicate tails, stolons, and the origin of the vertebrate trunk. Biol. Rev. 74: 177-198.

Lacalli TC. 2000. Larval budding, metamorphosis, and the evolution of life-history patterns in echinoderms. Invert. Biol. 119: 234-241.

Lacalli TC. 2001. New perspectives on the evolution of protochordate sensory and locomotory systems, and the origin of brains and heads. Phil. Trans. R. Soc. Lond. B 356: 15651572.

Lacalli TC, West JE. 1985. The nervous system of a pilidium larva: evidence from electron microscope reconstructions. Can. J. Zool. 63: 1909-1916.
Lacalli TC, Gilmour THC, West JE. 1990. Ciliary band innervation in the bipinnaria larva of Pisaster ochraceus. Phil. Trans. R. Soc. Lond. B 330: 371-390.

Lacalli TC, Gilmour THC, Kelly SJ. 1999. The oral nerve plexus in amphioxus larvae: function, cell types and phylogenetic significance. Proc. R. Soc. Lond. B 266: 1461-1470.

Ladurner P, Rieger R. 2000. Embryonic muscle development of Convoluta pulchra (Turbellaria - Acoelomorpha, Platyhelminthes). Dev. Biol. 222: 359-375.

Lammert V. 1991. Gnathostomulida. In: Harrison FW, Ruppert EE, eds. Microscopic anatomy of invertebrates. Vol. 4. Aschelminthes. New York: Wiley-Liss, 19-39.

Land J van der. 1975. Priapulida. In: Giese AC, Pearse JS, eds. Reproduction of marine invertebrates. New York: Academic Press, 55-65.

Land J van der, Nørrevang A. 1985. Affinities and intraphyletic relationships of the Priapulida. In: Conway Morris S, George JD, Gibson R, Platt HM, eds. The origins and relationships of lower invertebrates. Oxford: Clarendon Press, 261-273.

Lanzavecchia G, De Eguileor M, Valvassori R, Scari G. 1995. Body cavities of Nematomorpha. In: Lanzavecchia G, Valvassori R, Candia Carnevali MD, eds. Body cavities: function and phylogeny. Modena: Mucchi, 45-60.

Lee D-C, Bryant HN. 1999. A reconsideration of the coding of inapplicable characters: assumptions and problems. Cladistics 15: 373-378.

Lee MSY. 1998a. Similarity, parsimony and conjectures of homology: the chelonian shoulder girdle revisited. J. Evol. Biol. 11: 379-387.

Lee MSY. 1998b. Convergent evolution and character correlation in burrowing reptiles: towards a resolution of squamate relationships. Biol. J. Linn. Soc. 65: 369-453.

Lee MSY. 1999. Circularity, evolution, systematics... and circularity. J. Evol. Biol. 12: 724-734.

Lemburg C. 1995. Ultrastructure of the introvert and associated structures of the larvae of Halicryptus spinulosus (Priapulida). Zoomorphology 115: 11-29.

Lemburg C. 1998. Electron microscopical localization of chitin in the cuticle of Halicryptus spinulosus and Priapulus caudatus (Priapulida) using gold-labelled wheat germ agglutinin: phylogenetic implications for the evolution of the cuticle within the Nemathelminthes. Zoomorphology 118: 137-158.

Lemburg C, Schmidt-Rhaesa A. 1999. Priapulida. In: Knobil E, Neill JD, eds. Encyclopedia of Reproduction. New York: Academic Press, 1053-1058.

Lespinet $O$, Nederbragt AJ, Cassan M, Dictus WJAG, Van Loon AE, Adoutte A. 2002. Characterisation of two snail genes in the gastropod mollusk Patella vulgata. Implications for understandingthe ancestral function of the snail-related genes in Bilateria. Dev. Genes Evol. 212: 186-195.

Lester SM. 1988a. Settlement and metamorphosis of Rhabdopleura normani (Hemichordata: Pterobranchia). Acta Zool. 69: 111-120.

Lester SM. 1988b. Ultrastructure of adult gonads and development and structure of the larva of Rhabdopleura normani (Hemichordata: Pterobranchia). Acta Zool. 69: 95-109.

Lin M-F, Leise EM. 1997. Gangliogenesis in the prosobranch 
gastropod Ilyanassa obsoleta. J. Comp. Neurol. 374: 180193.

Lipscomb DL, Farris JS, Källersjo M, Tehle A. 1998. Support , ribosomal sequences and the phylogeny of the eukaryotes. Cladistics 14: 303-338.

Littlewood DTJ, Smith AB, Clough KA, Emson KH. 1997. The interrelationships of the echinoderm classes: morphological and molecular evidence. Biol. J. Linn. Soc. 61: 409438.

Littlewood DTJ, Telford MJ, Clough KA, Rohde K. 1998. Gnathostomulida - an enigmatic metazoan phylum from both morphological and molecular perspectives. Mol. Phyl. Evol. 9: 72-79.

Littlewood DTJ, Rohde K, Clough KA. 1999a. The interrelationships of all major groups of Platyhelminthes: phylogenetic evidence from morphology and molecules. Biol. J. Linn. Soc. 66: 75-114.

Littlewood DTJ, Rohde K, Bray RA, Herniou EA. 1999b. Phylogeny of the Platyhelminthes and the evolution of parasitism. Biol. J. Linn. Soc. 68: 257-287.

Littlewood DTJ, Olson PD. 2001. Small subunit rDNA and the Platyhelminthes: signal, noise, conflict and compromise. In: Littlewood DTJ, Bray RA, eds. Interrelationships of the Platyhelminthes. London: Taylor \& Francis, 262-278.

Littlewood DTJ, Olson PD, Telford MJ, Herniou EA, Riutort M. 2001. Elongation factor 1-alpha sequences alone do not assist in resolving the position of the Acoela within the Metazoa. Mol. Biol. Evol. 18: 437-442.

Locke JM. 2000. Ultrastructure of the statocyst of the marine enchytraeid Grania americana (Annelida: Clitellata). Invert. Biol. 119: 83-93.

Long JA, Stricker SA. 1991. Brachiopoda. In: Giese AC, Pearse $\mathrm{JS}$, Pearse VB, eds. Reproduction of marine invertebrates. Vol. VI. Echinoderms and lophophorates. California: The Boxwood Press, 47-84.

Loon AE van, Biggelaar JAM. van den. 1998. Changes in cell lineage specification elucidate evolutionary relations in Spiralia. Biol. Bull. 195: 367-369.

Lorenzen S. 1985. Phylogenetic aspects of pseudocoelomate evolution. In: Conway Morris S, George JD, Gibson R, Platt $\mathrm{HM}$, eds. The origins and relationships of lower invertebrates. Oxford: Clarendon Press, 210-223.

Lorenzen S. 1994. The phylogenetic systematics of freeliving nematodes. London: The Ray Society.

Lorenzen S. 1996a. Nematoda, Fadenwürmer. In: Westheide W, Rieger RM, eds. Spezielle Zoologie. Erster Teil: Einzeller und Wirbellose Tiere. Stuttgart: Gustav Fischer Verlag, 692710.

Lorenzen S. 1996b. Nemathelminthes (Aschelminthes). In: Westheide W, Rieger RM, eds. Spezielle Zoologie. Erster Teil: Einzeller und Wirbellose Tiere. Stuttgart: Gustav Fischer Verlag, 682-684.

Lorenzen S. 1996c. Rotatoria (Rotifera), Rädertiere. In: Westheide W, Rieger RM, eds. Spezielle Zoologie. Erster Teil: Einzeller und Wirbellose Tiere. Stuttgart: Gustav Fischer Verlag, 714-722.

Lorenzen S. 1996d. Priapulida, Priapswürmer. In: Westheide W, Rieger RM, eds. Spezielle Zoologie. Erster Teil: Einzeller und Wirbellose Tiere. Stuttgart: Gustav Fischer Verlag, 733735.

Lorenzen S. 1996e. Gastrotricha, Bauchhärlinge. In: Westheide W, Rieger RM, eds. Spezielle Zoologie. Erster Teil: Einzeller und Wirbellose Tiere. Stuttgart: Gustav Fischer Verlag, 685691.

Lorenzen S. 1996f. Acanthocephala, Kratzer. In: Westheide W, Rieger RM, eds. Spezielle Zoologie. Erster Teil: Einzeller und Wirbellose Tiere. Stuttgart: Gustav Fischer Verlag, 723728.

Lorenzen S. 1996g. Kinorhyncha. In: Westheide W, Rieger RM, eds. Spezielle Zoologie. Erster Teil: Einzeller und Wirbellose Tiere. Stuttgart: Gustav Fischer Verlag, 729-732.

Lundin K, Sterrer W. 2001. The Nemertodermatida. In: Littlewood DTJ, Bray RA, eds. Interrelationships of the Platyhelminthes. London: Taylor \& Francis, 24-27.

Lüter C. 2000. The origin of the coelom in Brachiopoda and its phylogenetic significance. Zoomorphology 120: 15-28.

Lüter C, Bartolomaeus T. 1997. The phylogenetic position of Brachiopoda - a comparison of morphological and molecular data. Zool. Scr. 26: 245-253.

MacBride EW. 1895. Sedwick's theory of the embryonic phase of ontogeny as an aid to phylogenetic theory. Quart. J. Microsc. Sci. 37: 325-342.

Mackey LY., Winnepenninckx B, Wachter T de, Emschermann P, Garey JR. 1996. 18S rRNA suggests that Entoprocta are protostomes, unrelated to Ectoprocta. J. Mol. Evol. 42: 552-559.

Mainitz M. 1989. Gnathostomulida. In: Adiyodi KG, Adiyodi RG, eds. Reproductive biology of invertebrates. Vol IV, Part $A$. Chicester: John Wiley \& Sons, 167-177.

Malakhov VV, Popelyaev IS, Galin SV. 1997. On the position of the Vestimentifera and Pogonophora in the system of the animal kingdom. Zool. Zhu. 76: 1336-1347.

Malakhov VV, Temereva EN. 1999. Embryonic development of the phoronid Phoronis ijimai (Lophophorata, Phoronida): two sources of the coelomic mesoderm. Dokl. Akad. Nauk 365: $574-576$.

Maley LE, Marshall CR. 1998. The coming of age of molecular systematics. Science 279: 505-506.

Manton SM. 1949. Studies on the Onychophora. VII. The early embryonic stages of Peripatopsis, and some general considerations concerning the morphology and phylogeny of the Arthropoda. Phil. Trans. R. Soc. Lond. B 233: 483-580.

Mariscal RN. 1975. Entoprocta. In: Giese AC, Pearse JS, eds. Entoprocts and lesser coelomates. New York: Academic Press, 1-41.

Markevich GI. 1993. SEM observations on Seison and phylogenetic relationships of the Seisonidae (Rotifera). Hydrobio logia 255/256: 513-520.

Mark Welch DB. 2001. Early contributions of molecular phylogenetics to understanding the evolution of Rotifera. Hydrobiologia 446/447: 315-322.

Marois R, Carew TJ. 1997. Ontogeny of serotonergic neurons in Aplysia californica. J. Comp. Neurol. 386: 477-490.

Martin P. 2001. On the origin of the Hirudinea and the demise of the Oligochaeta. Proc. R. Soc. Lond. B 268: 1089-1098.

Martin VJ. 1997. Cnidarians, the jellyfish and hydras. In: Gil- 
bert SF, Raunio AM, eds. Embryology. Constructing the organism.. Sunderland: Sinauer Associates, 57-86.

Martindale MQ, Henry J. 1997. Ctenophorans, the comb jellies. In: Gilbert SF, Raunio AM, eds. Embryology. Constructing the organism. Sunderland: Sinauer Associates, 87-111.

Martindale MQ, Henry JQ. 1998. The development of radial and biradial symmetry: the evolution of bilaterality. Amer. Zool. 38: 672-684.

Martindale MQ, Henry JQ. 1999. Intracellular fate mapping in a basal metazoan, the ctenophore Mnemiopsis leidyi, reveals the origins of mesoderm and the existence of indeterminate cell lineages. Dev. Biol. 214: 243-257.

Martindale MQ, Finnerty JR, Henry JQ. 2002. The Radiata and the evolutionary origins of the bilaterian body plan. Mol. Phyl. Evol. 24: 358-365.

Maslakova SA, Malakhov VV, Norenburg JL. 1999. Indirect development in the order Hoplonemertea (Phylum Nemertea). Amer. Zool. 39: 38A.

Maslakova SA, Norenburg JL. 2001. Trochophora larva is plesiomorphic for nemerteans: evidence for prototroch in a basal nemertean Carinoma tremaphoros (Phylum Nemertea, Palaeonemertea). Amer. Zool. 41: 1515-1516.

McHugh D. 1998. Deciphering metazoan phylogeny: the need for additional molecular data. Amer. Zool. 38: 859-866.

McHugh D. 2000. Molecular phylogeny of the Annelida. Can. J. Zool. 78: 1873-1884.

Mcllugh D, Rouse GW. 1998. Life history evolution of marine invertebrates: new views from phylogenetic systematics. TREE 13: 182-186.

McLean N. 1984. Amoebocytes in the lining of the body cavity and mesenteries of Priapulus caudatus (Priapulida). Acta Zool. 65: 75-78.

Meglitsch PA, Schram FR. 1991. Invertebrate Zoology. Oxford: Oxford University Press.

Mellon D. 1992. Connective tissue and supporting structures. In: Harrison FW, Humes AG, eds. Microscopic anatomy of invertebrates. Vol. 10. Decapod Crustacea. New York: WileyLiss, 77-116.

Melone G, Ricci C, Segers H, Wallace RL. 1998. Phylogenetic relationships of phylum Rotifera with emphasis on the families of Bdelloidea. Hydrobiologia 387/388: 101-107.

Michel C. 1988. X. Intestine and digestive glands. In: Westheide W, Hermans Co, eds. The ultrastructure of Polychaeta. Stuttgart: Gustav Fischer Verlag, 157-175.

Minelli A. 1995. Body cavities and body segmentation: problems of homology and phylogenetic reconstruction. In: Lanzavecchia G, Valvassori R, Candia Carnevali MD, eds. Body cavities: function and phylogeny. Modena: Mucchi, 6973.

Miner BG, Sanford E, Strathmann RR, Pernet B, Emlet RB. 1999. Functional and evolutionary implications of opposed bands, big mouths, and extensive oral ciliation in larval opheliids and echiurids (Annelida). Biol. Bull. 197: 14-25.

Monks S. 2001. Phylogeny of the Acanthocephala based on morphological characters. Syst. Parasitol. 48: 81-116.

Moore J, Willmer P. 1997. Convergent evolution in invertebrates. Biol. Rev. 72: 1-60.

Morse MP, Reynolds PD. 1996. Ultrastructure of the heart- kidney complex in smaller classes supports symplesiomorphy of molluscan coelomic characters. In: Taylor JD, ed. Origin and evolutionary radiation of the Mollusca. Oxford: Oxford University Press, 89-97.

Mukai H. 1982. Development of freshwater bryozoans (Phylactolaemata). In: Harrison FW, Cowden RR, eds. Developmental biology of freshwater invertebrates. New York: Alan R. Liss, Inc., 535-576.

Mukai H, Terakado K, Reed CG. 1997. Bryozoa. In: Harrison FW, Woollacott RM, eds. Microscopic anatomy of invertebrates. Vol. 13. Lophophorates, Entoprocta, and Cycliophora. New York: Wiley-Liss, 45-206.

Near TJ. 2002. Acanthocephalan phylogeny and the evolution of parasitism. Integ. And Comp. Biol. 42: 668-677.

Near TJ, Garey JR, Nadler SA. 1998. Phylogenetic relationships of the Acanthocephala inferred from $18 \mathrm{~S}$ ribosomal DNA sequences. Mol. Phyl. Evol. 10: 287-298.

Nebelsick M. 1993. Introvert, mouth cone, and nervous system of Echinoderes capitatus (Kinorhyncha, Cyclorhagida) and implications for the phylogenetic relationships of Kinorhyncha. Zoomsrphology 113: 211-232.

Nederbragt AJ, Lespinet Q, Van Wageningen S, Van Loon AE, Adoutte A, Dictus WJAG. 2002. A lophotrochozoan twist gene is expressed in the extomesoderm of the gastropod mollusk Patella vulgata. Evol. Devel. 4: 334-343.

Needham AE. 1989. Kinorhyncha. In: Adiyodi KG, Adiyodi RG, eds. Reproductive biology of invertebrates. Vol IV, Part A. Chicester: John Wiley \& Sons, 207-217.

Needham AE. 1990. Annelida - Clitellata. In: Adiyodi KG, Adiyodi RG, eds. Reproductive biology of invertebrates. Vol IV, Part B. Chicester: John Wiley \& Sons, 1-36.

Nelson DR. 1982. Developmental biology of the Tardigrada. In: Harrison FW, Cowden RR, eds. Developmental biology of freshwater invertebrates. New York: Alan R. Liss, Inc., 363-398.

Neuhaus B. 1994. Ultrastructure of alimentary canal and body cavity, ground pattern, and phylogenetic relationships of the Kinorhyncha. Microfauna Marina 9: 61-156.

Neuhaus B, Kristensen RM, Lemburg C. 1996. Ultrastructure of the cuticle of the Nemathelminthes and electron microscopical localization of chitin. Verh.Deutsch. Zool. Gesell. 89: 221 .

Neuhaus B, Bresciani J, Peters W. 1997a. Ultrastructure of the pharyngeal cuticle and lectin labelling with wheat germ agglutinin-gold conjugate indicating chitin in the pharyngeal cuticle of Oesophagostomum dentatum (Strongylida, Nematoda). Acta Zool. 78: 205-213.

Neuhaus B, Kristensen RM, Peters W. 1997b. Ultrastructure of the cuticle of Loricifera and demonstration of chitin using gold-labelled wheat germ agglutinin. Acta Zool. 78: 215-225.

Nezlin LP. 2000. Tornaria of hemichordates and other dipleurulatype larvae: a comparison. J. Zool. Syst. Evoo. Res. 38: 149$+56$.

Nickisch-Rosenegk M von, Brown WM, Boore JL. 2001. Complete sequence of the mitochondrial genome of the tapeworm Hymenolepis diminuta: gene arrangements indicate that platyhelminths are eutrochozoans. Mol. Biol. Evol. 18: 721730 . 
Niclsen C. 1971. Entoproct life-cycles and the entoproct/ ectoproct relationship. Ophelia 9, 209-341.

Nielsen C. 1985. Animal phylogeny in the light of the trochaea theory. Biol. J. Linn. Soc. 25: 243-299.

Nielsen C. 1987. Structure and function of metazoan ciliary bands and their phylogenetic significance. Acta Zool. 68: 205-262.

Nielsen C. 1990. Bryozoa, Ectoprocta. In: Adiyodi KG, Adiyodi RG, eds. Reproductive biology of invertebrates. Vol IV, Part B. Chicester: John Wiley \& Sons, 185-200.

Niclsen C. 1994. Larval and adult characters in animal phylogeny. Amer. Zool. 34: 492-501.

Nielsen C. 1991. The development of the brachiopod Crania (Neocrania) anomala (O. F. Müller) and its phylogenetic significance. Act. Zool. 72: 7-28.

Nielsen C. 1995. Animal evolution. Interrelationships of the living phyla. Oxford: Oxford University Press.

Nielsen C. 1997. The phylogenetic position of the Arthropoda. In: Fortey RA, Thomas RH, eds. Arthropod relationships. The Systematics Association Special Volume Series 55. London: Chapman \& Hall, 11-22.

Nielsen C. 1998a. Origin and evolution of animal life cycles. Biol. Rev. 73: 125-155.

Nielsen C. 1998b. Morphological approaches to phylogeny. Amer. Zool. 38: 942-952.

Niclsen C. 1999. Origin of the chordate central nervous system - and the origin of chordates. Dev. Genes Ev ol. 20: 198205.

Niclsen C. 2000. The phylogenetic position of Entoprocta and Ectoprocta. In: Herrera Cubilla A, Jackson JBC, eds. Proceedings of the 1lith International Bryozoology Association Conference. Balboa: Smithsonian Tropical Research Institute, 66-73.

Nielsen C. 2001. Animal evolution. Interrelationships of the living phyla. Second edition. Oxford: Oxford University Press.

Nielsen C. 2002a. The phylogenetic position of Entoprocta, Ectoprocta, Phoronida, and Brachiopoda. Integ. And Comp. Biol. 42: 685-691.

Nielsen C. 2002b. Ciliary filter-feeding structures in adult and larval gymnolaemate bryozoans. Invert. Biol. 121: 255-261.

Nielsen C, Norrevang A. 1985. The trochaea theory: an example of life cycle evolution. In: Conway Morris S, George JD, Gibson R, Platt HM, eds. The origins and relationships of lower invertebrates. Oxford: Clarendon Press, 28-41.

Nielsen C, Scharff N, Eibye-Jacobsen D. 1996. Cladistic analyses of the animal kingdom. Biol. J. Linn. Soc. 57: 385-410.

Nielsen C, Jespersen A. 1997. Entoprocta. In: Harrison FW, Woollacott RM, eds. Microscopic anatomy of invertebrates. Lophophorates, Entoprocta, and Cycliophora. New York: Wiley-Liss, 13-43.

Nielsen C, Riisgård HU. 1998. Tentacle structure and filterfeeding in Crisia eburnea and other cyclostomatous bryozoans, with a review of upstream-collecting mechanisms. Mar. Ecol. Prog. Ser. 16: 163-168.

Nielsen C, Meier R. 2002. What cell lineages tell us about the evolution of Spiralia remains to be seen. Evolution 56: 25542557.

Norenburg JL, Stricker SA. 2002. Phylum Nemertea. In: Young
CM, Sewell MA, Rice ME, eds. Atlas of marine invertebrate larvae. San Diego: Academic Press, 163-177.

Norrevang A, Van der Land J. 1989. Priapulida. In: Adiyodi KG, Adiyodi RG, eds. Reproductive biology of invertebrates. Vol IV, Part A. Chicester: John Wiley \& Sons, 259-262.

Nübler-Jung K, Arendt D. 1994. Is ventral in insects dorsal in vertebrates? A history of embryological arguments favouring axis inversion in chordate ancestors. Roux's Arch. Dev. Biol. 203: 357-366.

Nübler-Jung K, Arendt D. 1999. Dorsoventral axis inversion: enteropneust anatomy links vertebrates to chordates turned upside down. J. Zool. Syst. Evol. Res. 37: 93-100.

Okusu A. 2002. Embryogenesis and development of Epimenia babai (Mollusca Neomeniomorpha). Biol. Bull. 203: 87-103.

Olive PJW. 1985. Covariability of reproductive traits in marine invertebrates: implications for the phylogeny of the lower invertebrates. In: Conway Morris S, George JD, Gibson R, Platt HM, eds. The origins and relationships of lower invertebrates. Oxford: Clarendon Press, 42-59.

Page LR. 1994. The ancestral gastropod larval form is best approximated by hatching-stage opisthobranch larvae: evidence from comparative developmental studies. In: Wilson WH, Stricker SA, Shinn GL, eds. Reproduction and Development of Marine Invertebrates. Baltimore: Johns Hopkins University Press, 206-223.

Panchen AL. 2001. Etienne Geoffroy St.-Hilaire: father of "evodevo"? Evol. Devel. 3: 41-46.

Pardos F, Roldán C, Benito J, Emig CC. 1991. Fine structure of the tentacles of Phoronis australis Haswell (Phoronida, Lophophorata). Acta Zool. 72: 81-90.

Patterson C. 1982. Morphological characters and homology. In: Joysey KA, Friday AE, eds. Problems of phylogenetic reconstruction. London: Academic Press, 21-74.

Patterson C. 1988. Homology in classical and molecular biology. Mol. Biol. Evol. 5: 603-625.

Pawlowski J, Montoya-Burgos JI, Fahrni JF, Wüest J, Zaninetti L. 1996. Origin of the Mesozoa inferred from 18S ribosomal RNA gene sequences. Mol. Biol. Evol. 13: 11281132.

Pearse JS, Cameron RA. 1991. Echinodermata: Echinoidea. In: Giese AC, Pearse JS, Pearse VB, eds. Reproduction of marine invertebrates. Vol. VI. Echinoderms and lophophorates. California: The Boxwood Press, 514-662.

Peer Y van de, Wachter R. de 1997. Evolutionary relationships among the eukaryotic crown taxa taking into account site-to-site rate variation in $18 \mathrm{~S}$ rRNA. J. Mol. Evol. 45: 619630.

Peer Y van de, Baldauf SL, Doolittle WF, Meyer A. 2000. An updated and comprehensive rRNA phylogeny of (crown) eykaryotes based on rate-calibrated evolutionary distances. J. Mol. Evol. 51: 565-576.

Pernet B, Schroeder P. 1999. Opposed ciliary bands and larval feeding in a capitellid annelid. Amer. Zool. 39: 39A.

Peterson KJ, Davidson EH. 2000. Regulatory evolution and the origin of the bilaterians. Proc. Natl. Acad. Sci. USA.97: 4430-4433. .

Peterson KJ, Cameron RA, Tagawa K, Satoh N, Davidson EH. 1999. A comparative molecular approach to mesoder- 
mal patterning in basal deuterostomes: the expression pattern of Brachyury in the enteropneust hemichordate Ptychodera flava. Development 126: 85-95.

Peterson KJ, Cameron RA, Davidson EH. 2000a. Bilaterian origins: significance of new experimental observations. Dev. Biol. 219: 1-17.

Peterson KJ, Arenas-Mena C, Davidson EH. 2000b. The A/P axis in echinoderm ontogeny and evolution: evidence from fossils and molecules. Evol. Devel. 2: 93-101.

Peterson KJ, Eernisse DJ. 2001. Animal phylogeny and the ancestry of bilaterians: inferences from morphology and $18 \mathrm{~S}$ rDNA gene sequences. Evol. Devel. 3: 170-205.

Philippe H, Chenuil A, Adoutte A. 1994. Can the Cambrian explosion be inferred through molecular phylogeny? Development Suppl.: 15-25.

Pilger JF. 1978. Settlement and metamorphosis in the Echiura: a review. In: Chia F-S, Rice ME, eds. Settlement and metamorphosis of marine invertebrate larvae. New York: Elsevier, 103-112.

Pilger JF. 1993. Echiura. In: Harrison FW, Rice ME, eds. $M i-$ croscopic anatomy of invertebrates. Vol. 12. Onychophora, Chilopoda, and lesser Protostomata. New York: Wiley-Liss, 185-236.

Pilger JF. 1997. Sipunculans and echiurans. In: Gilbert SF, Raunio AM, eds. Embryology. Constructing the organism. Sunderland: Sinauer Associates, 167-188.

Pleijel F. 1995. On character coding for phylogeny reconstruction. Cladistics 11: 309-315.

Pogue MG, Mickevich MF. 1990. Character definitions and character state delineation: the bête noire of phylogenetic inference. Cladistics 6: 319-361.

Pollock LW. 1975. Tardigrada. In: Giese AC, Pearse JS, eds. Entoprocts and lesser coelomates. New York: Academic Press, 43-54.

Ponder WF, Lindberg DR. 1996. Gastropod phylogeny - challenges for the 90s. In: Taylor JD, ed. Origin and evolutionary radiation of the Mollusca. Oxford: Oxford University Press, 135-154.

Ponder WF, Lindberg DR. 1997. Towards a phylogeny of gastropod molluscs: an analysis using morphological characters. Zool. J. Linn. Soc. 119: 83-265.

Prenant M. 1959. Classe des Myzostomides. In: Grassé P-P, ed. Traité de Zoologie. Anatomie, Systématique, Biologie. Tome V. Annélides, Myzostomides, Sipunculiens, Echiuriens, Priapuliens, Endoproctes, Phoronidiens. Paris: Masson et Cie Éditeurs, 714-784.

Presley R, Horder TJ, Slípka J. 1996. Lancelet development as evidence of ancestral chordate structure. Isr. J. Zool. 42: 97-116.

Proctor HC. 1998. Indirect sperm transfer in arthropods: behavioral and evolutionary trends. Ann. Rev. Ent. 43:153-174.

Purschke G. 1996. Echiura (Echiurida), Igelwürmer. In: Westheide W, Rieger RM, eds. Spezielle Zoologie. Erster Teil: Einzeller und Wirbellose Tiere. Stuttgart: Gustav Fischer Verlag, 345-349.

Purschke G, Hessling R, Westheide W. 2000. The phylogenetic position of the Clitellata and the Echiura - on the problematic assessment of absent characters. J. Zool. Syst. Evol. Res. 38: 165-173.
Raff RA. 1998. Metazoan phylogeny. In: Anderson DT, ed. Invertebrate Zoology. Oxford: Oxford University Press, 416438.

Raff RA, Field KG, OIsen GJ, Giovannoni SJ, Lane DJ, Ghiselin MT, Pace NR, Raff EC. 1989. Metazoan phylogeny based on analysis of $18 \mathrm{~S}$ ribosomal RNA. In: Fernholm B, Bremer K, Jörnvall H, eds. The Hierarchy of life. Molecules and Morphology in phylogenetic analysis. Proceedings from Nobel Symposium 70, 1988. Amsterdam: Excerpta Medica/Elsevier, 247-260.

Raff RA, Marshall CR, Turbeville JM. 1994. Using DNA sequences to unravel the Cambrian radiation of the animal phyla. Ann. Rev. Ecol. Syst. 25: 351-375.

Raikova OI, Reuter M, Kotikova EA, Gustafsson MKS. 1998. A commissural brain! The pattern of 5-HT immunoreactivity in Acoela (Plathelminthes). Zoomorphology 118: 69-77.

Raikova OI, Reuter M, Justine JL. 2001. Contributions to the phylogeny and systematics of the Acoelomorpha. In: Littlewood DTJ, Bray RA, eds. Interrelationships of the Platyhelminthes. London: Taylor \& Francis, 13-23.

Raineri M. 2000. Early neurogenesis pattern in Patella coerulea (Patellogastropoda) and its possible phylogenetic implications. Malacologia 42: 131-148.

Raven CP. 1966. Morphogenesis. The analysis of molluscan development. Oxford: Pergamon Press.

Reed CG. 1991. Bryozoa. In: Giese AC, Pearse JS, Pearse VB, eds. Reproduction of marine invertebrates. Vol. VI. Echinoderms and lophophorates. California: The Boxwood Press, 85-245.

Reisinger E. 1972. Die Evolution des Orthogons der Spiralier und das Archicölomatenproblem. Z. zool. Syst. Evolutionsforsch. 10: 1-43.

Remane A. 1963a. Uber die Homologisierungsmöglichkeiten bei Verbindungsstrukturen (Muskeln, Blutgefässen, Nerven) und Hohlräumen. Zool. Anz. 166: 481-489.

Remane A. 1963b. The enterocoelic origin of the coelom. In: Dougherty EC, ed. The lower Metazoa. Comparative biology and phylogeny. Berkeley: University of California Press, 78-90.

Reuter M, Gustafsson MKS. 1995. The flatworm nervous system: pattern and phylogeny. In: Breidbach O, Kutsch W, eds, The nervous system of invertebrates: an evolutionary and comparative approach. Basel: Birkhäuser Verlag, 25-59.

Reuter M, Mäntylä K, Gustafsson MKS. 1998. Organization of the orthogon - main and minor nerve cords. Hydrobiologia 383: $175-182$.

Keuter M, Halton DW. 2001. Comparative neurobiology of Platyhelminthes. In: Littlewood DTJ, Bray RA, eds. Interrelationships of the Platyhelminthes. London: Taylor \& Francis, 239-249.

Shull AF. 1918. Cell inconstancy in Hydatina senta. J. Morph. 30: $455-464$.

Rice ME. 1975. Sipuncula. In: Giese AC, Pearse JS, eds. Entoprocts and lesser coelomates. New York: Academic Press, 67-127.

Rice ME. 1989. Sipuncula. In: Adiyodi KG, Adiyodi RG, eds. Reproductive biology of invertebrates. Vol IV. Part A. Chicester: John Wiley \& Sons, 263-280. 
Rice ME. 1993. Sipuncula. In: F. W. Harrison FW, Rice ME, eds. Microscopic anatomy of invertebrates. Vol. 12. Onychophora, Chilopoda, and lesser Protostomata. New York: Wiley-Liss, 237-325.

Rice SA. 1992. Polychaeta: spermatogenesis ands spermiogenesis. In: Harrison FW, Gardiner SL, eds. Microscopic anatomy of invertebrates. Vol. 7. Annelida. New York: Wiley-Liss, 129151.

Richter S. 2002. The Tetraconata concept: hexapod-crustacean relationships and the phylogeny of Crustacea. Org. Divers. Evol. 2: 217-237.

Riedl RJ. 1969. Gnathostomulida from America. Science 163: 445-452.

Rieger RM. 1976. Monociliated epidermal cells in Gastrotricha: significance for concepts of metazoan evolution. Z. zool. Syst. Evolutionsforsch. 14: 198-226.

Rieger RM. 1980. A new group of interstitial worms, Lobatocerebridae nov. fam. (Annelida) and its significance for metazoan phylogeny. Zoomorphologie 95: 41-84.

Rieger RM. 1981. Fine structure of the body wall, the nervous system, and the digestive system of the Lobatocerebridae Rieger (Annelida) and remarks to the organization of the gliuointerstitial systems in Annelida. J. Morph. 167: 139165.

Rieger RM. 1984. Evolution of the cuticle in the lower Metazoa. In: Bereiter-Hahn J, Matoltsy AG, Richards KS, eds. Biology of the integument. Vol. 1. Invertebrates. Berlin: SpringerVerlag, 389-399.

Rieger RM. 1985. The phylogenetic status of the acoelomate organization within the Bilateria: a histological perspective. In: Conway Morris S, George JD, Gibson R, Platt HM, eds. The origins and relationships of lower invertebrates. Oxford: Clarendon Press, 101-122.

Rieger RM. 1986a. Über den Ursprung der Bilateria: die Bedeutung der Ultrastrukturforschung für ein neues Verstehen der Metazoenevolution. Verh. Deutsch. Zool. Gesell. 79: 3150.

Rieger RM. 1986b. Asexual reproduction and the turbellarian archetype. Hydrobiologia 132: 35-45.

Rieger RM. 1988. Comparative ultrastructure and the Lobatocerebridae: keys to understand the phylogenetic relationship of Annelida and the acoelomates. In: Westheide W, Hermans $\mathrm{CO}$, eds. The ultrastructure of Polychaeta. Stuttgart: Gustav Fischer Verlag, 373-382.

Rieger RM. 1994a. Evolution of the "lower" Metazoa. In: Bengtson S, ed. Early life on earth. Nobel Symposium 84. New York: Columbia University Press, 475-488.

Rieger RM. 1994b. The biphasic life cycle - a central theme of metazoan evolution. Amer. Zool. 34: 484-491.

Rieger RM. 1996a. Triploblastische Eumetazoa, Bilateria. In: Westheide W, Rieger RM, eds. Spezielle Zoologie. Erster Teil: Einzeller und Wirbellose Tiere. Stuttgart: Gustav Fischer Verlag, 189-204.

Rieger RM. 1996b. Diploblastische Eumetazoa. In: Westheide W, Rieger RM, eds. Spezielle Zoologie. Erster Teil: Einzeller und Wirbellose Tiere. Stuttgart: Gustav Fischer Verlag, 131142.

Rieger RM. 1996c. Plathelminthes, Plattwürmer. In: Westheide
W, Rieger RM, eds. Spezielle Zoologie. Erster Teil: Einzeller und Wirbellose Tiere. Stuttgart: Gustav Fischer Verlag, 210258.

Rieger RM. 2001. Phylogenetic systematics of the Macrostomorpha. In: Littlewood DTJ, Bray RA, eds. Interrelationships of the Platyhelminthes. London: Taylor \& Francis, 28-38.

Rieger RM, Mainitz M. 1975. Comparative fine structure study of the body wall in Gnathostomulida and their phylogenetic position between Platyhelminthes and Aschelminthes. Z. zool. Syst. Evolutionsforsch. 15: 9-35.

Rieger RM, Lombardi J. 1987. Ultrastructure of coelomic lining in echinoderm podia: significance for concepts in the evolution of muscle and peritoneal cells. Zoomorphology 107: 191-208.

Rieger RM, Haszprunar G, Schuchert P. 1991a. On the origin of the Bilateria: traditional views and recent alternative concepts. In: Simonetta AM, Conway Morris S, eds. The early evolution of Metazoa and the significance of problematic taxa. Cambridge: Cambridge University Press, 107-112.

Rieger RM, Tyler S, Smith III JPS, Rieger GE. 1991b. Platyhelminthes: Turbellaria. In: Harrison FW, Bogitsh BJ, eds. Microscopic anatomy of invertebrates. Vol. 3. Platyhelminthes and Nemertinea. New York: Wiley-Liss, 7-140.

Rieger RM, Tyler S. 1995. Sister-group relationship of Gnathostomulida and Rotifera-Acanthocephala. Invert. Biol. 114: 186-188.

Rieger R, Ladurner P. 2001. Searching for the stem species of the Bilateria. Belg. J. Zool. (Suppl.) 131: 27-34.

Riisgård HU, Nielsen C, Larsen PS. 2000. Downstream collecting in ciliary suspension feeders: the catch-up principle. Mar. Ecol. Prog. Ser. 207: 33-51.

Robertis EM de, Sasai Y. 1996. A common plan for dorsoventral patterning in Bilateria. Nature 380: $37-40$.

Rohde K. 2001. Protonephridia as phylogenetic characters. In: Littlewood DTJ, Bray RA, eds. Interrelationships of the Platyhelminthes. London: Taylor \& Francis, 203-216.

Romer AS, Parsons TS. 1986. The vertebrate body. New York Harcourt Brace Jovanovich College Publishers.

Rosa R de, Grenier JK, Andreva T, Cook CE, Adoutte A, Akam M, Carroll SB, Balavoine G. 1999. Hox genes in brachiopods and priapulids and protostome evolution. $\mathrm{Na}$ ture 399: 772-776.

Rouse GW. 1999. Trochophore concepts: ciliary bands and the evolution of larvae in spiralian Metazoa. Biol. J. Linn. Soc. 66: 411-464.

Rouse GW. 2000a. The epitome of hand waving? Larval feeding and hypotheses of metazoan phylogeny. Evol. Devel. 2: 222-233.

Rouse GW. 2000b. Polychaetes have evolved feeding larvae numerous times. Bull. Mar. Sci. 67: 391-409.

Rouse GW. 2000c. Bias? What bias? The evolution of downstream larval-feeding in animals. Zool. Scr. 29: 213-236.

Rouse GW. 2001. A cladistic analysis of Siboglinidae Caullery, 1914 (Polychaeta, Annelida): formerly the phyla Pogonophora and Vestimentifera. Zool. J. Linn. Soc. 132: 55-80.

Rouse GW, Fitzhugh K. 1994. Broadcasting fables: is external fertilization really primitive? Sex, size, and larvae in sabellid polychaetes. Zool. Scr. 23: 271-312. 
Rouse GW, Fauchald K. 1995. The articulation of annelids. Zool. Scr. 24: 269-301.

Rouse GW, Fauchald K. 1997. Cladistics and polychaetes. Zool. Scr. 26: 139-204.

Rouse GW, Pitt K. 2000. Ultrastructure of the sperm of Catostylus mosaicus and Phyllorhiza punctata (Scyphozoa, Cnidaria): Implications for sperm terminology and the inference of reproductive mechanisms. Invert. Repro. Dev. 38: 23-34.

Rowe FWE, Anderson DT, Healy JM. 1991. Echinodermata: Concentricycloidea. In: Giese AC, Pearse JS, Pearse VB, eds. Reproduction of marine invertebrates. Vol. VI. Echinoderms and lophophorates. California: The Boxwood Press, 751-760.

Ruhberg H. 1990. Onychophora. In: Adiyodi KG, Adiyodi RG, eds. Reproductive biology of invertebrates. Vol IV, Part B. Chicester: John Wiley \& Sons, 61-76.

Ruiz-Trillo I, Riutort M, Littlewood DTJ, Herniou EA, Baguñà J. 1999. Acoel flatworms: earliest extant bilaterian metazoans, not members of Platyhelminthes. Science 283: 1919-1923.

Ruiz-Trillo I, Paps J, Loukota M, Ribera C, Jondelius U, Baguñà J, Riutort M. 2002. A phylogenetic analysis of myosin heavy chain type II sequences corroborates that Acoela and Nemertodermatida are basal bilaterians. Proc. Natl. Acad. Sci. USA 99: 11246-11251.

Ruppert EE. 1978. A review of metamorphosis of turbellarian larvae. In: Chia F-S, Rice ME, eds. Settlement and metamorphosis of marine invertebrate larvae. New York: Elsevier, 65-81.

Ruppert EE. 1982. Comparative ultrastructure of the gastrotrich pharynx and the evolution of myoepithelial foreguts in Aschelminthes. Zoomorphology 99: 181-220.

Ruppert EE. 1991a. Introduction to the aschelminth phyla: a consideration of mesoderm, body cavities, and cuticle. In: Harrison FW, Ruppert EE, eds. Microscopic anatomy of invertebrates. Vol. 4. Aschelminthes. New York: Wiley-Liss, 117.

Ruppert EE. 1991b. Gastrotricha. In: Harrison FW, Ruppert EE, eds. Microscopic anatomy of invertebrates. Vol. 4. Aschelminthes. New York: Wiley-Liss, 41-109.

Ruppert EE. 1994. Evolutionary origin of the vertebrate nephron. Amer. Zool. 34: 542-553.

Ruppert EE. 1997. Cephalochordata (Acrania). In: Harrison FW, Ruppert EE, eds. Microscopic anatomy of invertebrates. Vol. 15. Hemichordata, Chaetognatha, and the invertebrate chordates. New York: Wiley-Liss, 349-504.

Ruppert EE, Carle KJ. 1983. Morphology of metazoan circulatory systems. Zoomorphology 103: 193-208.

Ruppert EE, Balser EJ. 1986. Nephridia in the larvae of hemichordates and echinoderms. Biol. Bull. 171:188-196.

Ruppert EE, Smith PR. 1988. The functional organization of filtration nephridia. Biol. Rev. 63: 231-258.

Ruppert EE, Barnes RD. 1994. Invertebrate zoology. New York: Saunders College Publishing.

Ruppert EE, Cameron CB, Frick JE. 1999. Endostyle-like features of the dorsal epibranchial ridge of an enteropneust and the hypothesis of dorsal-ventral axis inversion in chordates. Invert. Biol. 118: 202-212.
Ruthensteiner B, Wanninger A, Haszprunar G. 2001. The protonephridial system of the tusk shell, Antalis entalis (Mollusca, Scaphopoda). Zoomorphology 121: 19-26.

Ruthmann A. 1996. Placozoa. In: Westheide W, Rieger RM, eds. Spezielle Zoologie. Erster Teil: Einzeller und Wirbellose Tiere. Stuttgart: Gustav Fischer Verlag, 121-124.

Saló E, Tauler J, Jimenez E, Bayascas JR, Gonzalez-Linares J, Garcia-Fernàndez J, Baguñà J. 2001. Hox and ParaHox genes in flatworms: characterization and expression. Amer. Zool. 41: 652-663.

Salvini-Plawen Lv. 1968. Die 'Funktions-Coelomtheorie' in der Evolution der Mollusken. Syst. Zool. 17: 192-208.

Salvini-Plawen Lv. 1978. On the origin and evolution of the lower Metazoa. Z. zool. Syst. Evolutionsforsch. 16: 40-88.

Salvini-Plawen Lv. 1980a. A reconsideration of systematics in the Mollusca (phylogeny and higher classification). Malacologia 19: 249-278.

Salvini-Plawen Lv. 1980b. Was ist eine Trochophora? Eine Analyse der Larventypen mariner Protostomier. Zool. Jb. Abt. Anat. 103: 389-423.

Salvini-Plawèn Lv. 1982. A paedomorphic origin og the oligomerous animals? Zool. Scr. 11: 77-81.

Salvini-Plawen Lv. 1985. Earrly evolution and the primitive groups. In: Trueman ER, Clarke MR, eds. The Mollusca. Vol. 10. Evolution. New York: Academic Press, 59-150.

Salvini-Plawen Lv. 1988. Annelida and Mollusca - a prospectus. In: Westheide W, Hermans $\mathrm{CO}$, eds. The ultrastructure of Polychaeta. Stuttgart: Gustav Fischer Verlag, 383-396.

Salvini-Plawen Lv. 1990. Origin, phylogeny and classification of the phylum Mollusca. Iberus 9: 1-33.

Salvini-Plawen Lv. 1998a. Morphologie: Haeckels GastraeaTheorie und ihre Folgen. Stapfia 56: 147-168.

Salvini-Plawen Lv. 1998b. The urochordate larva and archichordate organization: chordate origin and anagenesis revisited. J. Zool. Syst. Evol. Res. 36: 129-145.

Salvini-Plawen Lv. 2000. What is convergent/homoplastic in Pogonophora? J. Zool. Syst. Evol. Res. 38: 133-147.

Salvini-PIawen Lv, Splechtna H. 1979. Zur Homologie der Keimblätter. Z. zool. Syst. Evolutionsforsch. 17: 10-30.

Salvini-Plawen Lv, Bartolomaeus T. 1995. Mollusca: mesenchymata with a coelom. In: Lanzavecchia G, Valvassori R, Carnevali MD, eds. Body cavities: function and phylogeny. Modena: Mucchi, 75-92.

Salvini-Plawen Lv, Steiner G. 1996. Synapomorphies and plesiomorphies in higher classification of Mollusca. In: Taylor JD, ed. Origin and evolutionary radiation of the Mollusca. Oxford: Oxford University Press, 29-51.

Sandeman DC. 1982. Organization of the central nervous system. In: Atwood HL, Sandeman DC, eds. The biology of Crustacea. Volume 3. Neurobiology: structure and function. New York: Academic Press, 1-61.

Sanderson MJ, Purvis A, Henze C. 1998. Phylogenetic supertrees: assembling the trees of life. TREE 13: 105-109.

Saułnier-Michel C. 1992. Polychaeta: digestive system. In: Harrison FW, Gardiner SL, eds. Microscopic anatomy of invertebrates. Vol. 7. Annelida. New York: Wiley-Liss, 53-69.

Schaeffer B. 1987. Deuterostome monophyly and phylogeny. Evol. Biol. 21: 179-235. 
Schäfer W. 1996. Cnidaria, Nesseltiere. In: Westheide W, Rieger RM, eds. Spezielle Zoologie. Erster Teil: Einzeller und Wirbellose Tiere. Stuttgart: Gustav Fischer Verlag, 145-181.

Schaller F. 1979. Significance of sperm transfer and formation of spermatophores in arthropod phylogeny. In: Gupta AP, ed. Arthropod phylogeny. New York: Van Nostrand Reinhold Company, 587-608.

Scheltema AH, Tscherkassky M, Kuzirian AM. 1994. Aplacophora. In: Harrison FW, Kohn AJ, eds. Microscopic anatomy of invertebrates. Vol. 5. Mollusca I. New York: WileyLiss, 13-54.

Schierenberg E. 1997. Nematodes, the roundworms. In: Gilbert SF, Raunio AM, eds. Embryology. Constructing the organism. Sunderland: Sinauer Associates, 131-148.

Schmidt-Rhaesa A. 1996. Zur Morphologie, Biologie und Phylogenie der Nematomorpha. Ph.D. Thesis. Göttingen: Georg-August-Universität.

Schmidt-Rhaesa A. 1999. Nematomorpha. In: Knobil E, Neill JD, eds. Encyclopedia of Reproduction. New York: Academic Press, 333-340.

Schmidt-Rhaesa A. 2002. Two dimensions of biodiversity research exemplified by Nematomorpha and Gastrotricha. Integ. And Comp. Biol. 42: 633-640.

Schmidt-Rhaesa A, Bartolomaeus T, Lemburg C, Ehlers U, Garey JR. 1998. The position of the Arthropoda in the phylogenetic system. J. Morph. 238: 263-285.

Scholtz G. 1997. Cleavage, germ band formation and head segmentation: the ground pattern of the Euarthropoda. In: Fortey RA, Thomas RH, eds. Arthropod relationships. The Systematics Association Special Volume Series 55. London: Chapman \& Hall, 317-332.

Scholtz G. 2001. Evolution of developmental patterns in arthropods - the analysis of gene expression and its bearing on morphology and phylogenetics. Zoology 103: 99-111.

Scholtz G. 2002. The Articulata hypothesis - or what is a segment? Org. Divers. Evol. 2: 197-215.

Scholtz G, Mittmann B, Gerberding, M. 1998. The pattern of Distal-less expression in the mouthparts of crustaceans, myriapods and insects: new evidence for a gnathobasic mandible and the common origin of Mandibulata. Int. J. Dev. Biol. 42: 801-810.

Scholtz G, Wolff C. 2002. Cleavage, gastrulation, and germ disc formation of the amphipod Orchestia cavimana (Crustacea, Malacostraca, Peracarida). Contr. Zool. 71: 9-28.

Schram FR. 1991. Cladistic analysis of metazoan phyla and the placement of fossil problematica. In: Simonetta AM, Conway Morris S, eds. The early evolution of Metazoa and the significance of problematic taxa. Cambridge: Cambridge University Press, 35-46.

Schram FR, Ellis WN. 1994. Metazoan relationships: a rebuttal. Cladistics 10: 331- 337.

Schram FR, Jenner RA. 2001. The origin of Hexapoda: a crustacean perspective. Ann. Soc. Entomol. Fr. 37: 243-264.

Schroeder PC, Hermans CO. 1975. Annelida: Polychaeta. In: Giese AC, Pearse JS, eds. Reproduction of marine invertebrates. Vol. 3. Annelids and echiurans. New York: Academic Press, 1-213.

Schuchert P. 1990. The nephridium of the Bonellia viridis male (Echiura). Acta Zoologica 71: 1-4.
Schuchert P, Rieger RM. 1990. Ultrastructural observations on the dwarf male of Bonellia viridis. Acta Zool. 71: 5-16.

Schulze A. 2001. Comparative anatomy of excretory organs in vestimentiferan tube worms (Pogonophora, Obturata). $J$. Morph. 250: 1-11.

Schürmann F-W. 1987. Histology and ultrastructure of the onychophoran brain. In: Gupta AP, ed. Arthropod brain. Its Evolution, development, structure, and functions. John New York: Wiley \& Sons, Inc., 159-180.

Schürmann F-W. 1995. Common and special features of the nervous system of Onychophora: a comparison with Arthropoda, Annelida and some other invertebrates. In: Breidbach $\mathrm{O}$, Kutsch W, eds. The nervous system of invertebrates: an evolutionary and comparative approach. Basel: Birkhäuser Verlag, 139-158.

Shimek RL, Steiner G. 1997. Scaphopoda. In: Harrison FW, Kohn AJ, eds. Microscopic anatomy of invertebrates. Vol. $6 B$. Mollusca II. New York: Wiley-Liss, 719-781.

Shinn GL. 1994. Epithelial origin of mesodermal structures in arrowworms (Phylum Chaetognatha). Amer. Zool. 34: 523532.

Shinn GL. 1997. Chaetognatha. In: Harrison FW, Ruppert EE, eds. Microscopic anatomy of invertebrates. Vol. 15. Hemichordata, Chaetognatha, and the invertebrate chordates. New York: Wiley-Liss, 103-220.

Shirley TC, Storch V. 1999. Halicryptus higginsi n.sp (Priapulida) - a giant new species from Barrow, Alaska. Invert. Biol. 118: 404-413.

Siddall ME. 1998. Success of parsimony in the four-taxon case: Long-branch repulsion by likelihood in the Farris Zone. Cladistics 14: 209-220.

Siddall ME, Whiting MF. 1999. Long-branch abstractions. Cladistics 15: 9-24.

Siewing R. 1964. Zur Frage der Homologie ontogenetischer Prozesse und Strukturen. Verh. Deutsch. Zool. Gesell. Kiel 1964: 51-95.

Siewing R. 1969. Lehrbuch der vergleichenden Entwicklungsgeschichte der Tiere. Hamburg: Verlag Paul Parey.

Siewing R. 1972. Zur Deszendenz der Chordaten - Erwiderung

I und Versuch einer Geschichte der Archicoelomaten. Z. zool. Syst. Evolutionsforsch. 10: 267-291.

Siewing R. 1977. Mesoderm bei Ctenophoren. Z. zool. Syst. Evolutionsforsch. 15: 1-8.

Siewing R. 1979. Homology of cleavage-types? Fortschritte in der zoologischen Systematik und Evolutionsforschung 1: 718.

Smiley S. 1994. Holothuroidea. In: Harrison FW, Chia F-S, eds. Microscopic anatomy of invertebrates. Vol. 14. Echinodermata. New York: Wiley-Liss, 401-471.

Smiley S, McEuen FS, Chafee C, Krishnan S. 1991. Echinodermata: Holothuroidea. In: Giese AC, Pearse JS, Pearse VB, eds. Reproduction of marine invertebrates. Vol. VI. Echinoderms and lophophorates. California: The Boxwood Press, 664-750.

Smith JPS, Tyler S. 1986. Frontal organs in the Acoelomorpha (Turbellaria): ultrastructure and phylogenetic significance. Hydrobiologia 132: 71-78.

Smith PR. 1992. Polychaeta: excretory system. In: Harrison 
FW, Gardiner SL, eds. Microscopic anatomy of invertebrates. Vol. 7. Annelida. New York: Wiley-Liss, 71-108.

Smith PR, Ruppert EE. 1988. Nephridia: In: Westheide W, Hermans CO, eds. The ultrastructure of Polychaeta. Stuttgart: Gustav Fischer Verlag, 231-262.

Sorensen MV. 2000. An SEM study of the jaws of Haplognathia rosea and Rastrognathia macrostoma (Gnathostomulida), with a preliminary comparison with the rotiferan trophi. Acta Zool. 81: 9-16.

Sorensen MV. 2002. Phylogeny and jaw evolution in Gnathostomulida, with a cladistic analysis of the genera. Zool. Scr. 31: $461-480$.

Sorensen MV. 2003. Further structures in the jaw apparatus of Limnognathiamaerski (Micrognathozoa), with notes on the phylogeny of the Gnathifera. J. Morph. 255: 131-145.

Sørensen MV, Funch P, Willerslev E, Hansen AJ, Olesen J. 2000. On the phylogeny of the Metazoa in light of the Cycliophora and Micrognathozoa. Zoologischer Anzeiger 239: 297318 .

Sørensen MV, Sterrer W. 2002. New characters in the gnathostomulid mouth parts revealed by scannning electron microscopy. J. Morph. 253: 310-334.

Southward EC. 1975. Pogonophora. In: Giese AC, Pearse JS, eds. Entoprocts and lesser coelomates. New York: Academic Press, 129-156.

Southward EC. 1993. Pogonophora. In: Harrison FW, Rice ME, eds. Microscopic anatomy of invertebrates. Vol. 12. Onychophora, Chilopoda, and lesser Protostomata. New York: Wiley-Liss, 327-369.

Southward EC. 1999. Development of Perviata and Vestimentifera (Pogonophora). Hydrobiologia 402: 185-202.

Southward EC. 2000. Class Pogonophora. In: Beesley PL, Ross GJB, Glasby CJ, eds. Polychaetes and allies. The Southern synthesis. Fauna of Australia. Volume 4A Polychaeta, Myzostomida, Pogonophora, Echiura, Sipunculida. Collingwood: CSIRO Publishing, 331-351.

Stach T. 2000. Microscopic anatomy of developmental stages of Branchiostoma lanceolatum (Cephalochordata, Chordata). Bonn: Bonner Zoologische Monographien.

Stach T, Turbeville JM. 2002. Phylogeny of Tunicata inferred from molecular annd morphological characters. Mol. Phyl. Evol. 25: 408-428.

Starck D, Siewing R. 1980. Zur Diskussion der Begriffe Mesenchym und Mesoderm. Zool. Jb. Abt. Anat. 103: 374388.

Sterrer W. 1972. Systematics and evolution within the Gnathostomulida. Systematic Zoology 21: 151-173.

Sterrer W. 1996. Gnathostomulida, Kiefermäulchen. In: Westheide W, Rieger RM, eds. Spezielle Zoologie. Erster Teil: Einzeller und Wirbellose Tiere. Stuttgart: Gustav Fischer Verlag, 259-264.

Sterrer W, Mainitz M, Rieger RM. 1985. Gnathostomulida: enigmatic as ever. In: Conway Morris S, George JD, Gibson $\mathrm{R}$, Platt HM, eds. The origins and relationships of lower invertebrates. Oxford: Clarendon Press, 181-199.

Stocker L. 1998. The invertebrate Chordata, Hemichordata and Chaetognatha. In: Anderson DT, ed. Invertebrate Zoology. Oxford: Oxford University Press, 396-415.
Storch V. 1991. Priapulida. In: Harrison FW, Ruppert EE, eds. Microscopic anatomy of invertebrates. Vol. 4. Aschelminthes. New York: Wiley-Liss, 333-350.

Storch V. 1993. Pentastomida. In: Harrison FW, Rice ME, eds. Microscopic anatomy of invertebrates. Vol. 12. Onychophora, Chilopoda, and lesser Protostomata. New York: Wiley-Liss, 115-142.

Storch V, Ruhberg H. 1993. Onychophora. In: Harrison FW, Rice ME, eds. Microscopic anatomy of invertebrates. Onychophora, Chilopoda, and lesser Protostomata. New York: Wiley-Liss, 11-56.

Storch V, Kempendorf C, Higgins RP, Shirley TC, Jamieson BGM. 2000. Priapulida. In: Adiyodi KG, Adiyodi RG, Jamieson BGM, eds. Reproductive biology of invertebrates. Volume $I X$, part $B$. Progress in male gamete ultrastructure and phylogeny . New Delhi: Oxford \& IBH Publishing Co, $1-19$.

Strathmann RR. 1978. The evolution and loss of feeding larval stages of marine invertebrates. Evolution 32: 894-906.

Strathmann RR. 1988. Larvae, phylogeny and von Bear's law. In: Paul CRC, Smith $\mathrm{AB}$, eds. Echinoderm phylogeny and evolutionary bielogy. Oxford: Clarendon Press, 53-68.

Strathmann RR. 1993. Hypotheses on the origins of marine larvae. Ann. Rev. Evol. Syst. 24: 89-117.

Strathmann RR, Eernisse DJ. 1994. What molecular phylogenies tell us about the evolution of larval forms. Amer. Zool. 34: 502-512.

Stricker SA, Folsom MW. 1998. A comparative ultrastructural analysis of spermatogenesis in nemertean worms. Hydrobiologia 365: 55-72.

Strong EE, Lipscomb D. 1999. Character coding and inapplicable data. Cladistics 15: 363-371.

Sundberg P, Turbeville JM, Härlin MS. 1998. There is no support for Jensen's hypothesis of nemerteans as ancestors to the vertebrates. Hydrobiologia 365: 47-54.

Sundberg P, Turbeville JM, Lindh S. 2001. Phylogenetic relationships among higher nemertean (Nemertea) taxa inferred from 18S rDNA sequences. Mol. Phyl. Evol. 20: 327-334.

Swalla BJ. 2001. Phylogeny of the urochordates: implications for chordate evolution. In: Sawada H, Lambert CC, eds. Biology of ascidians. Tokyo: Springer Verlag, 219-224.

Swalla BJ, Cameron CB, Corley LS, Garey JR. 2000. Urochordates are monophyletic within the deuterostomes. Syst. Biol. 49: 52-64.

Sweet HC, Hodor PG, Ettensohn CA. 1999. The role of micromere signalling in Notch activation and mesoderm specification during sea urchin development. Development 126: 5255-5265.

Tagawa K, Humphreys T, Satoh N. 2000. T-brain expression in the apical organ of hermichordate tornaria larvae suggests its evolutionary link to the vertebrate forebrain. Mol. Dev. Evol. 288: 23-31.

Telford MJ. 2000. Turning Hox "signatures" into synapomonorphies. Evol. Devel. 2: 360-364.

Telford MJ. 2001. Embryology and developmental genes as clues to flatworm relationships. In: Littlewood DTJ, Bray RA, eds. Interrelationships of the Platyhelminthes. London: Taylor \& Francis, 279-291. 
Telford MJ, Holland PWH. 1993. The phylogenetic affinities of the chaetognaths: a molecular analysis. Mol. Biol. Evol. 10: 660-676.

Telford MJ, Lockyer AE, Cartwright-Finch C, Littlewood D'TJ. In press. Combined large and small subunit ribosomal RNA phylogenies support a basal position of the acoelomorph flatworms. Proc. R. Soc. Lond. B.

Terwilliger NB. 1998. Functional adaptations of oxygen-transport proteins. J. Exp. Biol. 201: 1085-1098.

Thollesson M, Norenburg JL. 2003. Ribbon worm relationships: a phylogeny of the phylum Nemertea. Proc. $R$. Soc. Lond. B. 270: 407-415.

Thomas MB. 1986. Embryology of the Turbellaria and its phylogenetic significance. Hydrobiologia 132: 105-115.

Thompson TE. 1960. The development of Neomenia carinata Tullberg (Mollusca Aplacophora). Proc. R. Soc. Lond. B. 153: 263-278.

Todd JA. 2000. The central role of ctenostomes in bryozoan phylogeny. In: Herrera Cubilla A, Jackson JBC, eds. Proceedings of the 1lth International Bryozoology Association Conference. Balboa: Smithsonian Tropical Research Institute, 104-135.

Turbeville JM. 1986. An ultrastructural analysis of coelomogenesis in the hoplonemertine Prosorhochmus americanus and the polychaete Magelona sp. J. Morph. 187: 51-60.

Turbeville JM. 1991. Nemertinea. In: Harrison FW, Bogitsh BJ, eds. Microscopic anatomy of invertebrates. Vol. 3. Platyhelminthes and Nemertinea. New York: Wiley-Liss, 285-328.

Turbeville JM. 1996. Nemertini, Schnurwürmer. In: Westheide W, Rieger RM, eds. Spezielle Zoologie. Erster Teil: Einzeller und Wirbellose Tiere. Stuttgart: Gustav Fischer Verlag, 265275 .

Turbeville JM. 2002. Progress in nemertean biology: development and phylogeny. Integ. and Comp. Biol. 42: 692-703.

Turbeville JM, Ruppert EE. 1985. Comparative ultrastucture and the evolution of nemertines. Amer. Zool. 25: 53-71.

Turbeville JM, Field KG, Raff RA. 1992. Phylogenetic position of phylum Nemertini, inferred from 18S rRNA sequences: molecular data as a test of morphological character homology. Mol. Biol. Evol. 9: 235-249.

Tyler S. 2001. The early worm. In: Littlewood DTJ, Bray RA, eds. Interrelationships of the Platyhelminthes. London: Taylor \& Francis, 3-12.

Tyler S, Hooge MD. 2001. Musculature of Gnathostomula armata Riedl 1971 and its ecological significance. Mar. Ecol. 22: 71-83.

Uchida T, Yamada M. 1968. Cnidaria. In: Kumé M, Dan K, eds. Invertebrate embryology. Belgrade: NOLIT Publishing House, 86-116.

Ulrich W. 1972. Die Geschichte des Archicoelomatenbegriffs und die Archicoelomatennatur der Pogonophoren. Z. zool. Syst. Evolutionsforsch. 10: 301-320.

Valentine JW. 1997. Cleavage patterns and the topology of the metazoan tree of life. Proc. Natl. Acad. Sci. USA 94: 80018005.

Venkatesh TV, Holland ND, Holland LZ, Su M-T, Bodmer R. 1999. Sequence and developmental expression of amphioxus AmphiNk2-1: insigths into the evolutionary origin of the vertebrate thyroid gland and forebrain. Dev. Genes Evol. 209: 254-259.

Verdonk NH, Biggelaar JAM van den 1983. Early development and the formation of the germ layers. In: Verdonk $\mathrm{NH}$, Biggelaar van den JAM, Tompa AS, eds. The Mollusca, Vol. 3. Development. New York: Academic Press, 91-122.

Verger-Bocquet M. 1992. Polychaeta: sensory structures. In: Harrison FW, Gardiner SL, eds. Microscopic anatomy of invertebrates. Vol. 7. Annelida. New York: Wiley-Liss, 181196.

Vickery MS, McClintock JB. 1998. Regeneration in metazoan larvae. Nature 394: 140.

Voltzow J. 1994. Gastropoda: Prosobranchia. In: Harrison FW, Kohn AJ, eds. Microscopic anatomy of invertebrates. Vol. 5. Mollusca I. New York: Wiley-Liss, 111-252.

Voronov DA. 1999. The embryonic development of Pontonema vulgare (Enoplida: Oncholaimidae) with a discussion of nematode phylogeny. Russ. J. Nematol. 7: 105-114.

Voronov DA, Panchin YV, Spiridonov SE. 1998. Nematode phylogeny and embryology. Nature 395: 28.

Wägele JW, Erikson E, Lockhart P, Misof B. 1999. The Ecdysozoa: artifact or monophylum? J. Zool. Syst. Evol. Res. 37: $211-223$.

Wägele JW, Misof B. 2001. On quality of evidence in phylogeny reconstruction: a reply to Zrzavý's defence of the 'Ecdysozoa' hypothesis. J. Zool. Syst. Evol. Res. 39: 165-176.

Walker MH. 1995. Relatively recent evolution of an unusual pattern of early embryonic development (long germ band?) in a South African onychophoran, Opisthopatus cinctipes Purcell (Onychophora: Peripatopsidae). Zool. J. Linn. Soc. 114: $61-76$.

Wallace RL, Ricci C, Melone G. 1996. A cladistic analysis of pseudocoelomate (aschelminth) morphology. Invert. Biol. 115: $104-112$.

Waloszek D, Dunlop, JA. 2002. A larval sea spider (Arthropoda: Pycnogonida) from the Upper Cambrian 'Orsten' of Sweden, and the phylogenetic position of pycnogonids. Palaeontology 45: 421-446.

Wanninger A, Ruthensteiner B, Lobenwein S, Salvenmoser W, Dictus WJA, Haszprunar G. 1999. Development of the musculature in the limpet Patella (Mollusca, Patellogastropoda). Dev. Genes Evol. 209: 226-238.

Wanninger A, Haszprunar G. 2002. Muscle development in Antalis entalis (Mollusca, Scaphopoda) and its significance for scaphopod relationships. J. Morph. 254: 53-64.

Watling L. 1999. Toward understanding the relationships of the peracaridan orders: the necessity of determining exact homologies. In: Schram FR, von Vaupel Klein JC, eds. Crustaceans and the biodiversity crisis. Leiden: Brill, 73-89.

Watson NA. 1999. Platyhelminthes. In: Adiyodi KG, Adiyodi $\mathrm{RG}$, eds. Reproductive biology of invertebrates. Volume IX, part A. Progress in male gamete ultrastructure and phylog. eny. New Delhi: Oxford \& IBH Publishing Co., 97-142.

Webber HH. 1977. Gastropoda: Prosobranchia. In: Giese AC, Pearse JS, eds. Reproduction of marine invertebrates. Vol. 4. Molluscs: gastropods and cephalopods. New York: Academic Press, 1-97.

Weiss MJ. 2001: Widespread hermaphroditism in freshwater gastrotrichs. Invert. Biol. 120: 308-341. 
Welsch U. 1995. Evolution of the body cavities in Deuterostomia. In: Lanzavecchia G, Valvassori R, Candia Carnevali MD, eds. Body cavities: function, and phylogeny, Modena: Mucchi, 111-134.

Welsch U, Storch V. 1982. Fine structure of the coelomic epithelium of Sagitta elegans (Chaetognatha). Zoomorphology 100: $217-222$.

Westheide W. 1986. The nephridia of the interstitial polychaete Hesionides arenaria and their phylogenetic significance (Polychaeta, Hesionidae). Zoomorphology 106: 35-43.

Westheide W. 1996. Annelida, Ringelwürmer. In: Westheide W, Rieger RM, eds. Spezielle Zoologie. Erster Teil: Einzeller und Wirbellose Tiere. Stuttgart: Gustav Fischer Verlag, 353-410.

Westheide W. 1997. The direction of evolution within the Polychaeta. J. Nat. Hist. 31: 1-15.

Westheide W, McHugh D, Purschke G, Rouse G. 1999. Systematization of the Annelida: different approaches. Hydrobiologia 402: 291-307.

Weygoldt P. 1996. Chelicerata, Spinnentiere. In: Westheide W, Rieger RM, eds. Spezielle Zoologie. Erster Teil: Einzeller und Wirbellose Tiere. Stuttgart: Gustav Fischer Verlag, 449497.

Wheeler WC, Cartwright P, Hayashi CY. 1993. Arthropod phylogeny: a combined approach. Cladistics 9: 1-39.

Wheeler WC, Whiting M, Wheeler QD, Carpenter JM. 2001. The phylogeny of the extant hexapod orders. Cladistics 17: 113-169.

Whittaker JR. 1997. Cephalochordates, the lancelets. In: Gilbert SF, Raunio AM, eds. Embryology. Constructing the organism. Sunderland: Sinauer Associates, 365-381.

Wilkinson M. 1995. A comparison of two methods of character construction. Cladistics 11: 297-308.

Williams A. 1997. Brachiopoda: introduction and integumentary system. In: Harrison FW, Woollacott RM, eds. Microscopic anatomy of invertebrates. Vol. 13. Lophophorates, Entoprocta, and Cycliophora. New York: Wiley-Liss, $237-$ 296.

Williams A, Carlson SJ, Brunton CHC, Holmer LE, Popov L. 1996. A supra-ordinal classification of the Brachiopoda. Phil. Trans. R. Soc. Lond. B. 351: 1171-1193.

Willmer P. 1990. Invertebrate relationships. Patterns in animal evolution. Cambridge: Cambridge University Press.

Wills MA. 1998. Cambrian and Recent disparity: the picture from priapulids. Paleobiology 24: 177-199.

Winchell CJ, Sullivan J, Cameron CB, Swalla BJ, Mallat J. 2002. Evaluating hypotheses of deuterostomie phylogeny and chordate evolution with new LSU and SSU ribosomal DNA data. Mol. Biol. Evol. 19: 762-776.

Wilson EB. 1898. Considerations on cell-lineage and ancestral reminiscence based on a re-examination of some points in the early development of annelids and polyclades. Ann. N.Y. Acad. Sci. 11: 1-27.

Wilson RA, Webster LA. 1974. Protonephridia. Biol. Rev. 49: 127-160.

Winnepenninckx B, Backeljau T, Mackey LY, Brooks JM, Wachter R de, Kumar S, Garey JR. 1995a. 18S rRNA data indicate that Aschelminthes are polyphyletic in origin and consist of at least three distinct clades. Mol. Biol. Evol. 12: 1132-1137.

Winnepennickx BMH, Backeljau T, Wachter R de 1995b. Phylogeny of protostome worms derived from 18S rRNA sequences. Mol. Biol. Evol. 12: 641-649.

Winnepenninckx BMH, Van De Peer Y, Backeljau T. 1998. Metazoan relationships on the basis of $18 \mathrm{~S}$ rRNA sequences: a few years later... Amer. Zool. 38: 888-906.

Wolff C, Scholtz G. 2002. Cell lineage, axis formation, and the origin of germ layers in the amphipod crustacean Orchestia cavimana. Dev. Biol. 250: 44-48.

Woollacott RM, Harrison FW. 1997. Introduction. In: Harrison FW, Woollacott RM, eds. Microscopic anatomy of invertebrates. Vol. 13. Lophophorates, Entoprocta, and Cycliophora. New York: Wiley-Liss, 1-11.

Wray GA. 1997. Echinoderms. In: Gilbert SF, Raunio AM, eds. Embryology. Constructing the organism. Sunderland: Sinauer Associates, 309-329.

Wright KA. 1991. Nematoda. In: Harrison FW, Ruppert EE, eds. Microscopic anatomy of invertebrates. Vol. 4. Aschelminthes. New York: Wiley-Liss, 111-195.

Younossi-Hartenstein A, Hartenstein V. 2000. The embryonic development of the polyclad flatworm Imogine mcgrathi. Dev. Genes Evol. 210: 383-398.

Zimmer RL. 1991. Phoronida. In: Giese AC, Pearse JS, Pearse VB, eds. Reproduction of marine invertebrates. Vol. VI. Echinoderms and lophophorates. California: The Boxwood Press, $1-45$.

Zimmer RL. 1997. Phoronids, brachiopods, and bryozoans, the lophophorates. In: Gilbert SF, Raunio AM, eds. Embryology. Constructing the organism. Sunderland: Sinauer Associates, 279-305.

Zrzavý J. 2001. Ecdysozoa versus Articulata: clades, artifacts, prejudices. J. Zool. Syst. Evol. Res. 39: 159-163.

Zrzavý J. 2003. Gastrotricha and metazoan phylogeny. Zool. Scr. 32: 61-81.

Zrzavý J, Mihulka S, Kepka P, Bezdek A. 1998. Phylogeny of the Metazoa based on morphological and 18S ribosomal DNA evidence. Cladistics 14: 249-285.

Zrzavý J, Hypsa V, Tietz DF. 2001. Myzostomida are not annelids. Molecular and morphological support for a clade of animals with anterior sperm flagella. Cladistics 17: 170-198.

Received: 7 November 2002 